\title{
A equação de Hamilton-Jacobi no \\ contexto das funções generalizadas
}

\author{
Roseli Fernandez
}

Tese Apresentada ao

INSTITUTO DE MATEMÁTICA E ESTATÍSTICA

da

\section{UNIVERSIDADE DE SÃO PAULO}

para a obtenção do grau de doutor em matemática

Área de concentração: Análise

Orientador: Prof. Dr. Alfredo Jorge Aragona Vallejo

São Paulo, agosto de 1996. 
Este exemplar corresponde à redação final da tese devidamente corrigida e defendida por Roseli Fernandez e aprovada pela Comissão Julgadora.

São Paulo, 22 de novembro de 1996.

\section{BANCA EXAMINADORA}

Prof. Dr. Alfredo Jorge Aragona Vallejo (orientador) IME-USP

Prof. Dr. Paulo Domingos Cordaro IME-USP

Prof. Dr. Francisco Villarreal Alvarado FEIS-UNESP

Prof. Dr. Adalberto Panobianco Bergamasco UFSCar

Prof. Dra. Hebe de Azevedo Biagioni IMECC-UNICAMP 
À minha afilhada e sobrinha,

Camila Maria

e aos meus sobrinhos,

Erika Maria

e Leonardo. 


\section{RESUMO}

Neste trabalho estudamos a equação de Hamilton-Jacobi com uma condição inicial dada, no contexto das funções generalizadas de Colombeau. Estabelecemos um teorema de existência de soluções, utilizando uma técnica baseada no método, clássico das características, e alguns resultados parciais sobre a unicidade. Esta técnica nos levou a obter, no contexto das aplicações generalizadas, alguns resultados sobre a inversibilidade global dessas aplicações e também sobre equações diferenciais ordinárias.

\section{ABSTRACT}

In this work we study the Hamilton-Jacobi equations with a given initial condition, in the framework of Colombeau's generalized functions. We have obtained a theorem on existence of solutions and also some partial results on uniqueness. We use a technique which has been based on the classical method of characteristics. This technique, in the framework of generalized mappings, has led us to obtain some results on global invertibility of these mappings and also on ordinary differential equations. 


\section{Índice}

Introdução

Índice de símbolos e notações iv

Capítulo 1. A Álgebra das Funções Generalizadas de Colombeau

1.1. Definições e aígumas propriedades básicas $\ldots \ldots \ldots \ldots \ldots \ldots \ldots \ldots \ldots \ldots \ldots \ldots$

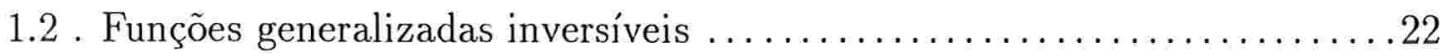

Capítulo 2 . Equação de Hamiitton-Jacobi : Existência de soluções

2.1. Um teorema de existência de soluções para a equação de

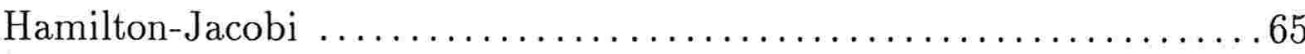

Capítulo 3 . Equação de Hamilton-Jacobi : Unicidade de soluções

3.1. Equações diferenciais ordinárias : existência e unicidade de

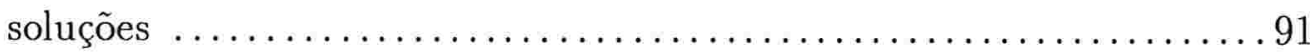

3.2 . Equações diferenciais ordinárias : dependência das soluções

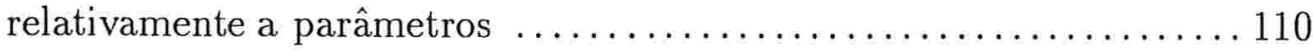

3.3 . Equação de Hamilton-Jacobi : unicidade de soluções $\ldots \ldots \ldots \ldots \ldots . . \ldots 146$

Apêndice 163

Bibliografia 


\section{Introdução}

A equação de Hamilton-Jacobi é uma equação diferencial parcial de primeira ordem, não linear, onde não aparece a função incógnita $u$. Esta equação aparece freqüentemente na Mecância Analítica.

O Professor J. F. Colombeau nos sugeriu o estudo, no contexto das funções generalizadas, da equação de Hamilton-Jacobi com uma condição inicial dada. No contexto clássico (isto é, que não usa a Teoria de Colombeau) sabemos que: dados I um intervalo aberto de $\mathbb{R}$ com $0 \in I, \Omega$ e $\Omega^{\prime}$ abertos de $\mathbb{R}^{n}, H \in \mathcal{C}^{\infty}\left(I \times \Omega \times \Omega^{\prime} ; \mathbb{R}\right)$ e $f \in \mathcal{C}^{\infty}(\Omega ; \mathbb{R})$ existe um aberto $W$ de $I \times \Omega$ com $V=\left\{z \in \mathbb{R}^{n}:(0, z) \in W\right\} \neq \emptyset$ e existe uma função $u \in \mathcal{C}^{\infty}(W ; \mathbb{R})$ que é uma solução para a equação de Hamilton-Jacobi

$$
\frac{\partial u}{\partial t}+H\left(t, x_{1}, \ldots, x_{n}, \frac{\partial u}{\partial x_{1}}, \ldots, \frac{\partial u}{\partial x_{n}}\right)=0
$$

e satisfaz a condição inicial

$$
\left.u\right|_{\{0\} \times V}=\left.f\right|_{V} .
$$

Neste trabalho procuramos obter, sob certas condições, um resultado análogo para o caso generalizado, isto é, admitindo $H$ e $f$ funções generalizadas. O problema de determinar, neste caso, o aberto $W$ e a função $u$ foi denominado, no capítulo 2, problema $\mathbf{H J}$ e $u$ foi chamada solução para o problema $\mathbf{H J}$.

Dentre os métodos clássicos existentes procuramos adaptar o método das características. Este método consiste em transformar um problema de E.D.P. com uma condição inicial dada em um problema de E.D.O. com uma certa condição inicial. Os métodos mais modernos, como por exemplo os que utilizam a noção de solução de viscosidade (introduzida por M. G. Crandall e P. L. Lions em [8] e reformulada por M. G. Crandall, L. C. Evans e P. L. Lions em [9]), foram deixados, propositadamente, para um estudo posterior, visto que envolvem trabalho com desigualdades. 
Para adaptar o método clássico das características definimos na seção 2.1, o conjunto $\mathcal{S}\left(I, \Omega, \Omega^{\prime}, H, f, J, W\right)$ no qual os elementos $(X, P)$ são tais que a derivada em relação à primeira variável das aplicações generalizadas $X$ e $P$ satisfazem um certo sistema de E.D.O. envolvendo funções generalizadas (este sistema, no caso clássico, é chamado sistema Hamiltoniano) e a aplicação generalizada $Y$, definida a partir de $X$, é uma aplicação generalizada inversível. No capítulo 2 vimos que, se $\mathcal{S}\left(I, \Omega, \Omega^{\prime}, H, f, J, W\right) \neq \emptyset$, então existe uma função generalizada que é solução para o problema HJ. Portanto para dar exemplos de funções $H$ e $f$ para as quais o problema $\mathbf{H J}$ admite solução basta procurar $H$ e $f$ satisfazendo $\mathcal{S}\left(I, \Omega, \Omega^{\prime}, H, f, J, W\right) \neq \emptyset$. Isto nos levou a obter alguns resultados sobre funções generalizadas inversíveis e sobre E.D.O. no contexto das funções generalizadáas.

Na seção 1.2 apresentamos uma condição necessária para que uma aplicação generalizada seja inversível e obtivemos alguns resultados que garantem, sob certas hipóteses, a inversibilidade global de uma função generalizada. Estes resultados foram usados nas seções 2.1 e 3.3 .

Nas seções 3.1 e 3.2 apresentamos os resultados que otivemos sobre E.D.O. no contexto das funções generalizadas. Os resultados utilizados neste trabalho estão em 3.2. A seção 3.1, apesar de não ser usada aqui, nos parece interessante para um estudo introdutório de E.D.O. no contexto das funções generalizadas.

Após obter um teorema de existência de soluções para o problema $\mathbf{H J}$ nos preocupamos com a unicidade de soluções. Obtivemos respostas parciais à esta questão. Estes resultados estão na seção 3.3 .

Na seção 1.1 apresentamos alguns resultados, a maioria deles conhecidos, relacionados com a teoria das funções generalizadas que nos serão utéis.

No final da seção 3.3 enunciamos algumas questões que surgiram durante a elaboração deste trabalho e para as quais, até o momento, não temos resposta.

No apêndice apresentamos, conforme sugestão da Banca Examinadora, alguns resultados para a equação 


$$
\frac{\partial u}{\partial t}+H\left(t, x_{1}, \ldots, x_{n}, u, \frac{\partial u}{\partial x_{1}}, \ldots, \frac{\partial u}{\partial x_{n}}\right)=0
$$

com a condição inicial

$$
\left.u\right|_{\{0\} \times V}=\left.f\right|_{V},
$$

para o caso generalizado.

Aqui trabalhamos cum a álgebra simplificada de Colombeau 'que foi denotada por $\mathcal{G}(\Omega ; \mathbb{R})$, sendo $\Omega$ um aberto de $\left.\mathbb{R}^{n}\right)$. O teorema de existência de soluções para o problema $\mathbf{H J}$, aqui apresentado, nos parece verdadeiro na teoria geral, mas como para fornecer exemplos precisávamos inverter funções generalizadas preferimos nos restringir à teoria simplicada. A adaptação destes resultados para a teoria geral ficou para um estudo posterior. Trabalhos sobre E.D.P. de primeira ordem no contexto da teoria das funções generalizadas envolvendo a definição de uma nova álgebra, a álgebra $\mathcal{G}(\Omega \times X ; \mathbb{R})$, sendo $\Omega$ um aberto de $\mathbb{R}^{n}$ e $X$ um subconjurnto fechado de $\mathbb{R}^{m}$, ou a definição de derivada regularizada podem ser encontrados em [6] e [7], respectivamente.

Neste trabalho a palavra "aberto" sempre significará aberto não vazio.

Desejo agradecer ao Prof. Jorge Aragona pela dedicada orientação e ao Prof. J. F. Colombeau pela sugestão do tema objeto desta tese.

Obrigada Senhor pela companhia.

Roseli Fernandez 


\section{Índice de símbolos e notações}

$K \subset \subset \Omega$

$|\alpha|$, onde $\alpha=\left(\alpha_{1}, \ldots, \alpha_{n}\right) \in \mathbb{N}^{n}$

$\partial^{\alpha}$, onde $\alpha=\left(\alpha_{1}, \ldots, \alpha_{n}\right) \in \mathbb{N}^{n}$

$1 /$

$\mathcal{E}_{M}\left[\Omega ; \mathbb{R}^{p}\right]$

$\mathcal{N}\left[\Omega ; \mathbb{R}^{p}\right]$

$\mathcal{G}\left(\Omega ; \mathbb{R}^{p}\right)$

$\mathcal{E}_{M}(\mathbb{R})$

$\mathcal{N}(\mathbb{R})$

$\overline{\mathbb{I R}}^{s}$

$\mathcal{G}_{*}\left(\Omega ; \Omega^{\prime}\right)$

$1_{\Omega}$

$J f$

$I_{a}$

$I_{a}\left(t_{0}\right)$

$\pi$ e $\pi_{j}, 1 \leq i \leq n$

problema $\mathbf{H J}$

$\mathcal{S}\left(I, \Omega, \Omega^{\prime}, H, f, J, W\right)$

$\widehat{f}$ limitada em $\Omega$

$\widehat{f}$ tem a propriedade (CLL) em $\Omega$

$\widehat{f}$ tem a propriedade (LLL) em $A$

$\mathcal{E}\left(t_{o}, a, \tau, I, \Omega, \Omega^{\prime}, W\right)$
K é uma parte compacta de $\Omega$

$\alpha_{1}+\alpha_{2}+. .+\alpha_{n}$

$\frac{\partial^{|\alpha|}}{\partial x_{1}^{\alpha_{1}} \ldots \partial x_{n}^{\alpha_{n}}}$

indica fim ou ausência de demonstração

1.1.7

1.1.8

1.1.9

1.1 .16

1.1 .16

1.1 .16

1.1 .18

início da seção 1.2

1.2 .11

1.2 .21

3.1 .8

início do capítulo 2

início do capítulo 2

2.1.1

3.1.1.i

3.1.1.ii

3.1.4

3.2 .1 


\section{Capítulo 1}

\section{A Álgebra das Funções \\ Generalizadas de Colombeau}

Neste capítulo apresentamos alguns resultados relacionados com a álgebra das funções generalizadas. Para facilitar a leitura colocamos na seção 1.1 alguns resultados básicos da teoria das funções generalizadas que nos serão úteis. Na seção 1.2 estabelec€mos alguns fatos sobre aplicações generalizadas inversíveis e fornecemos alguns exemplos que serão utilizados no capítulo 2 .

\subsection{Definições e algumas propriedades básicas}

Além de fixar as notações, apresentamos algumas definições e resultados, a maioria deles conhecidos, adaptados aos nossos propósitos. Destacamos os resultados relacionados com composta de aplicações generalizadas e com o conceito de inverso multiplicativo. A definição de aplicação composta aqui apresentada foi baseada em [2] e a caracterização de inversos multiplicativos que usamos pode ser encontrada em [14]. Omitimos a prova de algumas proposições por estarem demonstradas em [1], [2], [5] ou [14].

1.1.1 Definição. Seja $\Omega$ um aberto de $\mathbb{R}^{n}$. Denotaremos por $\mathcal{E}[\Omega ; \mathbb{R}]$ o conjunto das funçôes $\hat{f}$, definidas em $] 0,1] \times \Omega$ e com valores em $\mathbb{R}$, tais que $\widehat{f}(\varepsilon,.) \in \mathcal{C}^{\infty}(\Omega ; \mathbb{R})$, para 
todo $\varepsilon \in] 0,1]$.

1.1.2 Notação. Se $\Omega$ é um aberto de $\mathbb{R}^{n}, \alpha \in N^{n}$ e $\hat{f} \in \mathcal{E}[\Omega ; \mathbb{R}]$, escreveremos $\partial^{\alpha} \hat{f}(\varepsilon, x)$ no lugar de $\left(\partial^{\alpha}(\hat{f}(\varepsilon,)).(x)\right.$, e denotaremos por $\partial^{\alpha} \hat{f}$ a função definida em $\left.] 0,1\right] \times \Omega$ por $\left(\partial^{\alpha} \hat{f}\right)(\varepsilon, x)=\partial^{\alpha} \hat{f}(\varepsilon, x)$.

1.1.3 Definição. Seja $\Omega$ um aberto de $\mathbb{R}^{n}$. Denotaremos por $\mathcal{E}_{M}[\Omega ; \mathbb{R}]$ o conjunto das funções $\hat{f}$, definidas em $] 0,1] \times \Omega$ e com valores em $\mathbb{R}$, tais que

(i) $\hat{f} \in \mathcal{E}[\Omega ; \mathbb{R}]$;

(ii) dados quaisquer $K \subset \subset \Omega$ e $\alpha \in \mathbb{N}^{n}$, existem $N \in \mathbb{N}, c>0$ e $\left.\left.\eta \in\right] 0,1\right]$ tais que $\sup \left\{\left|\partial^{\alpha} \widehat{f}(\varepsilon, x)\right|: x \in K\right\} \leq c \varepsilon^{-N}$, para todo $\left.\varepsilon \in\right] 0, \eta[$.

Um elemento de $\mathcal{E}_{M}[\Omega ; \mathbb{R}]$ é chamado função moderada em $\Omega$ a valores em $\mathbb{R}$.

O conjunto $\mathcal{E}_{M}[\Omega ; \mathbb{R}]$ munido das operações usuais de soma e produto de funções e produto de número real por função é uma $\mathbb{R}$-álgebra.

É fácil verificar que se $\alpha \in \mathbb{N}^{n}$ e $\hat{f} \in \mathcal{E}_{M}[\Omega ; \mathbb{R}]$, então $\partial^{\alpha} \hat{f} \in \mathcal{E}_{M}[\Omega ; \mathbb{R}]$.

1.1.4 Definição. Seja $\Omega$ um aberto de $\mathbb{R}^{n}$. Denotaremos por $\mathcal{N}[\Omega ; \mathbb{R}]$ o conjunto das funçôes $\hat{f}$, definidas em $] 0,1] \times \Omega$ e com valores em $\mathbb{R}$, tais que

(i) $\hat{f} \in \mathcal{E}[\Omega ; \mathbb{R}]$;

(ii) dados quaisquer $K \subset \subset \Omega, \alpha \in \mathbb{N}^{n}$ e $q \in \mathbb{N}$, existem $c>0$ e $\left.\left.\eta \in\right] 0,1\right]$ tais que $\sup \left\{\left|\partial^{\alpha} \hat{f}(\varepsilon, x)\right|: x \in K\right\} \leq c \varepsilon^{q}$, para todo $\left.\varepsilon \in\right] 0, \eta[$.

Um elemento de $\mathcal{N}[\Omega ; \mathbb{R}]$ é chamado função nula em $\Omega$ a valores em $\mathbb{R}$.

O conjunto $\mathcal{N}[\Omega ; \mathbb{R}]$ é um ideal da álgebra $\mathcal{E}_{M}[\Omega ; \mathbb{R}]$.

É fácil verificar que se $\alpha \in \mathbb{N}^{n}$ e $\hat{f} \in \mathcal{N}[\Omega ; \mathbb{R}]$, então $\partial^{\alpha} \hat{f} \in \mathcal{N}[\Omega ; \mathbb{R}]$.

1.1.5 Definição. Seja $\Omega$ um aberto de $\mathbb{R}^{n}$. Denotaremos por $\mathcal{G}(\Omega ; \mathbb{R})$ a álgebra quociente de $\mathcal{E}_{M}[\Omega ; \mathbb{R}]$ por $\mathcal{N}[\Omega ; \mathbb{R}]$. 
Um elemento de $\mathcal{G}(\Omega ; \mathbb{R})$ é chamado função generalizada em $\Omega$ a valores em $\mathbb{R}$.

Se $f \in \mathcal{G}(\Omega ; \mathbb{R})$ denotaremos por $\hat{f}$ um representante de $f$ e por $\partial^{\alpha} f$ a classe de $\partial^{\alpha} \hat{f}$ em $\mathcal{G}(\Omega ; \mathbb{R})$, sendo $\alpha \in \mathbb{N}^{n}$.

1.1.6 Definição. Sejam $\Omega$ um aberto de $\mathbb{R}^{n}$ e $p \in \mathbb{N}$ com $p>1$. Denotaremos por $\mathcal{E}\left[\Omega ; \mathbb{R}^{p}\right]$ o conjunto das funç̃̃es vetoriais $\hat{f}=\left(\hat{f}_{1}, \ldots, \hat{f}_{p}\right)$, definidas em $\left.] 0,1\right] \times \Omega$ e com valores em $\mathbb{R}^{p}$, tais que $\hat{f}_{i} \in \mathcal{E}[\Omega ; \mathbb{R}]$ para todo $1 \leq i \leq p$.

1.1.7 Definição. Sejam $\Omega$ um aberto de $\mathbb{R}^{n}$ e $p \in \mathbb{N}$ com $p>1$. Denotaremos por $\mathcal{E}_{M}\left[\Omega ; \mathbb{R}^{p}\right]$ o conjunto das funções vetoriais $\hat{f}=\left(\widehat{f}_{1}, \ldots, \hat{f}_{p}\right)$, definidas em $\left.] 0,1\right] \times \Omega$ e com valores em $\mathbb{R}^{p}$, tais que $\widehat{f}_{i} \in \mathcal{E}_{M}[\Omega ; \mathbb{R}]$ para todo $1 \leq i \leq p$.

Se $\widehat{f}=\left(\widehat{f}_{1}, \ldots, \widehat{f}_{p}\right) \in \mathcal{E}\left[\Omega ; \mathbb{R}^{p}\right]$, é claro que são equivalentes:

(a) $\hat{f} \in \mathcal{E}_{M}\left[\Omega ; \mathbb{R}^{p}\right]$

(b) dados quaisquer $K \subset \subset \Omega$ e $\alpha \in \mathbb{N}^{n}$, existem $N \in \mathbb{I}, c>0$ e $\left.\left.\eta \in\right] 0,1\right]$ tais que $\sup \left\{\left|\partial^{\alpha} \widehat{f}_{i}(\varepsilon, x)\right|: x \in K\right\} \leq c \varepsilon^{-N}$, para todo $\left.\varepsilon \in\right] 0, \eta[$ e $1 \leq i \leq p$.

Um elemento de $\mathcal{E}_{M}\left[\Omega ; \mathbb{R}^{p}\right]$ é chamado aplicação moderada em $\Omega$ a valores em $\mathbb{R}^{p}$.

O conjunto $\mathcal{E}_{M}\left[\Omega ; \mathbb{R}^{p}\right]$ munido das operações usuais é um $\mathbb{R}$-espaço vetorial.

É fácil verificar que $\mathcal{E}_{M}\left[\Omega ; \mathbb{R}^{p}\right]=\bigoplus_{i=1}^{p} \mathcal{E}_{M}\left[\Omega ; \mathbb{R}_{i}\right]$, sendo $\mathbb{R}_{i}=\mathbb{R}$ para todo $1 \leq i \leq p$.

1.1.8 Definição. Sejam $\Omega$ um aberto de $\mathbb{R}^{n}$ e $p \in \mathbb{N}^{*}$. Denotaremos por $\mathcal{N}\left[\Omega ; \mathbb{R}^{p}\right]$ o conjunto das funçôes vetoriais $\hat{f}=\left(\widehat{f}_{1}, \ldots, \widehat{f}_{p}\right)$, definidas em $\left.] 0,1\right] \times \Omega$ e com valores em $\mathbb{R}^{p}$, tais que $\hat{f}_{i} \in \mathcal{N}[\Omega ; \mathbb{R}]$ para todo $1 \leq i \leq p$.

Se $\widehat{f}=\left(\widehat{f}_{1}, \ldots, \widehat{f}_{p}\right) \in \mathcal{E}\left[\Omega ; \mathbb{R}^{p}\right]$, é claro que são equivalentes:

(a) $\hat{f}=\left(\hat{f}_{1}, \ldots, \hat{f}_{p}\right) \in \mathcal{N}\left[\Omega ; \mathbb{R}^{p}\right]$

(b) dados quaisquer $K \subset \subset \Omega, \alpha \in \mathbb{N}^{n}$ e $q \in \mathbb{N}$, existem $c>0$ e $\left.\left.\eta \in\right] 0,1\right]$ tais que $\sup \left\{\left|\partial^{\alpha} \widehat{f}_{i}(\varepsilon, x)\right|: x \in K\right\} \leq c \varepsilon^{q}$, para todo $\left.\varepsilon \in\right] 0, \eta[$ e $1 \leq i \leq p$. 
Um elemento de $\mathcal{N}\left[\Omega ; \mathbb{R}^{p}\right]$ é chamado aplicação nula em $\Omega$ a valores em $\mathbb{R}^{p}$.

É fácil verificar que $\mathcal{N}\left[\Omega ; \mathbb{R}^{p}\right]=\bigoplus_{i=1}^{p} \mathcal{N}\left[\Omega ; \mathbb{R}_{i}\right]$, sendo $\mathbb{R}_{i}=\mathbb{R}$ para todo $1 \leq i \leq p$.

O conjunto $\mathcal{N}\left[\Omega ; \mathbb{R}^{p}\right]$ é um $\mathbb{R}$-subespaço vetorial de $\mathcal{E}_{M}\left[\Omega ; \mathbb{R}^{p}\right]$.

1.1.9 Definição. Sejam $\Omega$ um aberto de $\mathbb{R}^{n}$ e $p \in \mathbb{N}$ com $p>1$. Denotaremos por $\mathcal{G}\left(\Omega ; \mathbb{R}^{p}\right)$ o espaço vetorial quociente de $\mathcal{E}_{M}\left[\Omega ; \mathbb{R}^{p}\right]$ por $\mathcal{N}\left[\Omega ; \mathbb{R}^{p}\right]$.

Um elemento de $\mathcal{G}\left(\Omega ; \mathbb{R}^{p}\right)$ é chamado aplicaçâo generalizada em $\Omega$ a valores em $\mathbb{R}^{p}$.

É fácil verificar que a aplicação $\varphi$ definida em $\mathcal{G}\left(\Omega ; \mathbb{R}^{p}\right)$ e com valores em $\bigoplus_{i=1}^{p} \mathcal{G}\left(\Omega ; \mathbb{R}_{i}\right)$, sendo $\mathbb{R}_{i}=\mathbb{R}$ para todo $1 \leq i \leq p$, dada por

$$
\varphi(f)=\varphi\left(\left(\hat{f}_{1}, \ldots, \hat{f}_{p}\right)+\mathcal{N}\left[\Omega ; \mathbb{R}^{p}\right]\right)=\left(\widehat{f}_{1}+\mathcal{N}[\Omega ; \mathbb{R}], \ldots, \hat{f}_{p}+\mathcal{N}[\Omega ; \mathbb{R}]\right)
$$

é um isomorfismo entre espaços vetoriais. Sendo assim, identificaremos $\mathcal{G}\left(\Omega ; \mathbb{R}^{p}\right)$ com $\bigoplus_{i=1}^{p} \mathcal{G}\left(\Omega ; \mathbb{R}_{i}\right)$, sendo $\mathbb{R}_{i}=\mathbb{R}$ para todo $1 \leq i \leq p$ e escreveremos, quando for conveniente, $f$ ao invés de $\varphi(f)$, e assim um elemento $f \in \mathcal{G}\left(\Omega ; \mathbb{R}^{p}\right)$ será indicado por $f=\left(f_{1}, \ldots, f_{p}\right)$, sendo $f_{i} \in \mathcal{G}(\Omega ; \mathbb{R})$ para todo $1 \leq i \leq p$, e um representante de $f$ será indicado por $\left(\widehat{f}_{1}, \ldots, \hat{f}_{p}\right)$, sendo $\hat{f}_{i}$ um representante de $f_{i}$ em $\mathcal{G}(\Omega ; \mathbb{R})$ para todo $1 \leq i \leq p$.

Usaremos, principalmente no capítulo 2, a seguinte notação:

1.1.10 Notação. Se $f \in \mathcal{G}\left(\Omega ; \mathbb{R}^{p}\right) e\left\{s_{1}, \ldots, s_{r}\right\} \subset \mathbb{N}^{*}$ é tal que $\sum_{i=1}^{r} s_{i}=p$, escreveremos, quando for conveniente, $f=\left(g_{1}, \ldots, g_{r}\right)$ com $g_{i} \in \mathcal{G}\left(\Omega ; \mathbb{R}^{s_{i}}\right)$ para todo $1 \leq i \leq r$, ao invés de $f=\left(f_{1}, \ldots, f_{p}\right)$ com $f_{j} \in \mathcal{G}(\Omega ; \mathbb{R})$ e $1 \leq j \leq p$.

1.1.11 Proposição. Se $\Omega$ é um aberto de $\mathbb{R}^{n}, f \in \mathcal{G}\left(\Omega ; \mathbb{R}^{p}\right)$ e $\hat{g}$ e $\hat{h}$ são representantes de $f$, então $\lim _{\varepsilon \downarrow 0}(\widehat{g}(\varepsilon, x)-\widehat{h}(\varepsilon, x))=0$, para todo $x \in \Omega$.

Demonstração. Basta observar que o resultado é verdadeiro para $\mathcal{G}(\Omega ; \mathbb{R})($ ver $[1])$. //

1.1.12 Proposição. Sejam $\Omega$ um aberto de $\mathbb{R}^{n}, V$ aberto de $\mathbb{R}^{n}$ com $V \subset \Omega$ ef $\in$ $\mathcal{G}\left(\Omega ; \mathbb{R}^{p}\right)$. Se $\hat{f}_{1}$ e $\widehat{f}_{2}$ são representantes de $f$, então as aplicaçôes definidas em $\left.] 0,1\right] \times V$ por 


$$
\widehat{h}(\varepsilon, x)=\hat{f}_{1}(\varepsilon, x) \quad \text { e } \quad \widehat{h}_{1}(\varepsilon, x)=\widehat{f}_{1}(\varepsilon, x)-\widehat{f}_{2}(\varepsilon, x),
$$

pertencem, respectivamente, a $\mathcal{E}_{M}\left[V ; \mathbb{R}^{p}\right]$ e a $\mathcal{N}\left[V ; \mathbb{R}^{p}\right]$.

Demonstração. Basta observar que se $K \subset \subset V$, então $K \subset \subset \Omega$. //

1.1.13 Proposição. Sejam $\Omega^{\prime}$ um aberto de $\mathbb{R}^{n+1}, t_{o} \in \mathbb{R} \operatorname{com} \Omega^{\prime \prime}=\left\{z \in \mathbb{R}^{n}:\left(t_{o}, z\right) \in\right.$ $\left.\Omega^{\prime}\right\} \neq \emptyset, W$ um aberto de $\mathbb{R}^{n}$ com $W \subset \Omega^{\prime \prime}$ e $g \in \mathcal{G}\left(\Omega^{\prime} ; \mathbb{R}^{p}\right)$. Se $\widehat{g}_{1}$ e $\widehat{g}_{2}$ são representantes de $g$, então as aplicações definidas em $] 0,1] \times W$ por

$$
\hat{l}(\varepsilon, x)=\widehat{g}_{1}\left(\varepsilon, t_{o}, x\right) \quad e \quad \hat{l}_{1}(\varepsilon, x)=\widehat{g}_{1}\left(\varepsilon, t_{o}, x\right)-\widehat{g}_{2}\left(\varepsilon, t_{o}, x\right),
$$

pertencem, respectivamente, a $\mathcal{E}_{M}\left[W ; \mathbb{R}^{p}\right]$ e a $\mathcal{N}\left[W ; \mathbb{R}^{p}\right]$.

Demonstração. Basta observar que, se $K \subset \subset W$, então $\left\{t_{o}\right\} \times K \subset \subset \Omega^{\prime} . / /$

Com as duas proposições anteriores faz sentido a seguinte definição:

1.1.14 Definição. Sejam $\Omega^{\prime}$ um aberto de $\mathbb{R}^{n+1}, \Omega$ aberto de $\mathbb{R}^{n}, V$ aberto de $\mathbb{R}^{n}$ com $V \subset \Omega, t_{o} \in \mathbb{R}$ com $\Omega^{\prime \prime}=\left\{z \in \mathbb{R}^{n}:\left(t_{o}, z\right) \in \Omega^{\prime}\right\} \neq \emptyset$ e W aberto de $\mathbb{R}^{n}$ com $W \subset \Omega^{\prime \prime}, f \in \mathcal{G}\left(\Omega ; \mathbb{R}^{p}\right)$ e $g \in \mathcal{G}\left(\Omega^{\prime} ; \mathbb{R}^{p}\right)$. Sejam $\widehat{f}_{1}$ e $\hat{h}$ como em 1.1 .12 e $\widehat{g}_{1}$ e $\hat{l}$ como em 1.1.13. Denotaremos por $\left.f\right|_{V}$ a classe de $\hat{h}$ em $\mathcal{G}\left(V ; \mathbb{R}^{p}\right)$ e por $\left.g\right|_{\left\{t_{o}\right\} \times W}$ a classe de $\hat{l}$ em $\mathcal{G}\left(W ; \mathbb{R}^{p}\right)$.

A proposição abaixo será usada no capítulo 2 .

1.1.15 Proposição. Sejam $\Omega$ um aberto de $\mathbb{R}^{n}, I$ um intervalo aberto de $\mathbb{R}, t_{0} \in I$ e $g \in \mathcal{G}(I \times \Omega ; \mathbb{R})$. Denotando por $(t, x)=\left(t, x_{1}, \ldots, x_{n}\right)$ um ponto genérico de $I \times \Omega$ tem-se que, se

(i) $\left.g\right|_{\left\{t_{o}\right\} \times \Omega}=0$ em $\mathcal{G}(\Omega ; \mathbb{R})$;

(ii) $\frac{\partial g}{\partial t}=0 \mathrm{em} \mathcal{G}(I \times \Omega ; \mathbb{R})$,

então $g=0$ em $\mathcal{G}(I \times \Omega ; \mathbb{R})$. 
Demonstração. Seja $\widehat{g}$ um representante de $g$ e tomemos $K \subset \subset I \times \Omega, \alpha=\left(\alpha_{0}, \alpha_{1}, \ldots, \alpha_{n}\right) \in$ $\mathbb{N}^{n+1}$ e $q \in \mathbb{N}$.

Provaremos que existe $c>0$ e $\eta \in] 0,1]$ tais que

$$
\left.\left|\partial^{\alpha} \widehat{g}(\varepsilon, t, x)\right| \leq c \varepsilon^{q} \text {, para todo } \varepsilon \in\right] 0, \eta[\text { e }(t, x) \in K
$$

Sejam $L \subset \subset \Omega, a$ e $b$ números reais tais que $t_{o} \in[a, b] \subset I$ e $K \subset[a, b] \times L$. Suponhamos, em primeiro lugar, $\alpha_{0} \neq 0$ e seja $\beta=\left(\alpha_{0}-1, \alpha_{1}, \ldots \alpha_{n}\right)$.

Como $\frac{\partial g}{\partial t}=0$ em $\mathcal{G}(I \times \Omega ; \mathbb{R})$ existem $c_{1}>0$ e $\left.\left.\eta_{1} \in\right] 0,1\right]$ tais que

$$
\left.\sup \left\{\left|\partial^{\beta}\left(\frac{\partial \widehat{g}}{\partial t}\right)(\varepsilon, t, x)\right|:(t, x) \in K\right\} \leq c_{1} \varepsilon^{q}, \text { para todo } \varepsilon \in\right] 0, \eta_{1}[
$$

e portanto

$$
\left.\left|\partial^{\alpha} \widehat{g}(\varepsilon, t, x)\right| \leq c_{1} \varepsilon^{q}, \text { para todo }(\varepsilon, t, x) \in\right] 0, \eta_{1}[\times K,
$$

o que prova (1), no caso $\alpha_{0} \neq 0$.

Suponhamos $\alpha_{0}=0$. Como $\left.g\right|_{\left\{t_{o}\right\} \times \Omega}=0 \operatorname{em~} \mathcal{G}(\Omega ; \mathbb{R})$ temos que para $\beta=\left(\alpha_{1}, \ldots, \alpha_{n}\right)$, existem $c_{2}>0$ e $\left.\left.\eta_{2} \in\right] 0,1\right]$ tais que

$$
\left.\left|\partial^{\alpha} \widehat{g}\left(\varepsilon, t_{o}, x\right)\right|=\left|\partial^{\beta} \widehat{g}\left(\varepsilon, t_{o}, x\right)\right| \leq c_{2} \varepsilon^{q}, \quad \text { para todo } x \in L \text { e } \varepsilon \in\right] 0, \eta_{2}[\text {. }
$$

Como $\frac{\partial g}{\partial t}=0$ em $\mathcal{G}(I \times \Omega ; \mathbb{R})$, existem $c_{3}>0$ e $\left.\eta_{3} \in\right] 0, \eta_{2}[$ tais que

$$
\left.\left|\partial^{\alpha}\left(\frac{\partial \widehat{g}}{\partial t}\right)(\varepsilon, t, x)\right| \leq c_{3} \varepsilon^{q}, \text { para todo }(t, x) \in[a, b] \times L \text { e } \varepsilon \in\right] 0, \eta_{3}[\text {. }
$$

Fixemos $(\bar{t}, \bar{x}) \in K \subset[a, b] \times L$. Então, pelo Teorema do Valor Médio, temos que

$$
\partial^{\alpha} \hat{g}(\varepsilon, \bar{t}, \bar{x})=\partial^{\alpha} \hat{g}\left(\varepsilon, t_{o}, \bar{x}\right)+\frac{\partial}{\partial t}\left(\partial^{\alpha} \widehat{g}\right)(\varepsilon, s, \bar{x})\left(\bar{t}-t_{o}\right),
$$

para algum $s$ entre $t_{o}$ e $\bar{t}$.

Sejam $c=c_{2}+c_{3}(b-a)$ e $\eta=\eta_{3}$. Então de (2), (3) e (4) concluímos que

$$
\left.\left|\partial^{\alpha} \widehat{g}(\varepsilon, t, x)\right| \leq c \varepsilon^{q}, \quad \text { para todo }(t, x) \in K \text { e } \varepsilon \in\right] 0, \eta[
$$

o que prova (1), no caso $\alpha_{0}=0$.

Portanto $g=0$ em $\mathcal{G}(I \times \Omega ; \mathbb{R})$. //

A seguir vamos lemıbrar a definição de número generalizado. 
1.1.16 Definição. Seja $\mu$ uma função definida em ]0,1] a valores em $\mathbb{R}$. Dizemos que $\mu$ pertence a $\mathcal{E}_{M}(\mathbb{R})$ se, e somente se, existem $N \in \mathbb{N}, c>0$ e $\left.\left.\eta \in\right] 0,1\right]$ tais que

$$
\left.|\mu(\varepsilon)| \leq c \varepsilon^{-N}, \text { para todo } \varepsilon \in\right] 0, \eta[
$$

É claro que $\mathcal{E}_{M}(\mathbb{R})$ é uma $\mathbb{R}$-álgebra.

Dizemos que $\mu$ pertence a $\mathcal{N}(\mathbb{R})$ se, e somente se, dado qualquer $q \in N$ existem $c>0$ e $\eta \in] 0,1]$ tais que

$$
\left.|\mu(\varepsilon)| \leq c \varepsilon^{q}, \text { para todo } \varepsilon \in\right] 0, \eta[
$$

É claro que $\mathcal{N}(\mathbb{R})$ é um ideal de $\mathcal{E}_{M}(\mathbb{R})$.

Denotaremos por $\overline{\mathbb{R}}$ a álgebra quociente de $\mathcal{E}_{M}(\mathbb{R})$ por $\mathcal{N}(\mathbb{R})$.

Se $s \in \mathbb{N}^{*}$ e $x_{o}=\left(x_{1}, \ldots, x_{s}\right) \in \overline{\mathbb{R}}^{s}$ dizemos que $\widehat{x}_{o}=\left(\widehat{x}_{1}, \ldots, \widehat{x}_{s}\right) \in\left(\mathcal{E}_{M}(\mathbb{R})\right)^{s}$ é um representante de $x_{o}$ se, e somente se, $x_{i}=\widehat{x}_{i}+\mathcal{N}(\mathbb{R})$ para todo $1 \leq i \leq s$.

Um elemento de $\mathcal{E}_{M}(\mathbb{R})$ é chamado elernerto moderado.

Um elemento de $\mathcal{N}(\mathbb{R})$ é chamado elemento nulo.

Um elemento de $\overline{\mathbb{R}}$ é chamado de número generalizado.

Um elemento de $\overline{\mathbb{R}}^{s}$ é chamado vetor generalizado, sendo $s \in \mathbb{N}$ e $s>1$.

1.1.17 Definição. Sejam $\Omega$ um aberto de $\mathbb{R}^{n}$ e $p \in \Omega$. Se $f \in \mathcal{G}(\Omega ; \mathbb{R})$ denotaremos por $f(p)$ a classe do elemento moderado $\mu(\varepsilon)=\hat{f}(\varepsilon, p)$ em $\overline{\mathbb{R}}$, sendo $\hat{f}$ um representante qualquer de $f$. Se $g=\left(g_{1}, \ldots, g_{s}\right) \in \mathcal{G}\left(\Omega ; \mathbb{R}^{s}\right)$ denotaremos por $g(p)$ o vetor generalizado $\left(g_{1}(p), \ldots, g_{s}(p)\right) \in \overline{\mathbb{R}}^{s}$.

1.1.18 Definição. Sejam $\Omega$ um aberto de $\mathbb{R}^{n}$ e $\Omega^{\prime}$ um aberto de $\mathbb{R}^{p}$. Denotaremos por $\mathcal{G}_{*}\left(\Omega ; \Omega^{\prime}\right)$ o conjunto das aplicaçôes generalizadas $f \in \mathcal{G}\left(\Omega ; \mathbb{R}^{p}\right)$ e tais que existe um representante $\widehat{f}$ de $f$ satisfazendo:

(i) dado qualquer $K \subset \subset \Omega$, existem $K^{\prime} \subset \subset \Omega^{\prime}$ e $\left.\left.\eta \in\right] 0,1\right]$ tais que $\hat{f}(] 0, \eta[\times K) \subset K^{\prime}$. Convém observar que, se $\Omega^{\prime}=\mathbb{R}^{p}$, então a asserção (i) é equivalente a 
(i') dado qualquer $K \subset \subset \Omega$, existem $M>0$ e $\eta \in] 0,1]$ tais que $\|\widehat{f}(\varepsilon, x)\| \leq M$, para todo $(\varepsilon, x) \in] 0, \eta[\times K$,

e portanto $\mathcal{G}_{*}(\Omega ; \mathbb{R})$ é uma $\mathbb{R}$-álgebra.

1.1.19 Proposição Sejam $\Omega$ um aberto de $\mathbb{R}^{n}, \Omega^{\prime}$ um aberto de $\mathbb{R}^{p}$ e $f \in \mathcal{G}_{*}\left(\Omega ; \Omega^{\prime}\right)$. Então todo representante de $f$ satisfaz 1.1.18.i .

Demonstração. Sejam $\hat{f}=\left(\widehat{f}_{1}, \ldots, \widehat{f}_{p}\right)$ um representante de $f$ satisfazendo 1.1.18.i e $\widehat{g}=\left(\widehat{g}_{1}, \ldots, \widehat{g}_{p}\right) \in \mathcal{E}_{M}\left[\Omega ; \mathbb{R}^{p}\right] \operatorname{com} \hat{f}-\hat{g} \in \mathcal{N}\left[\Omega ; \mathbb{R}^{p}\right]$.

Seja $K \subset \subset \Omega$.

Usando 1.1.18.i existem $K^{\prime} \subset \subset \Omega^{\prime}$ e $\left.\left.\eta \in\right] 0,1\right]$ tais que

$$
\{\hat{f}(\varepsilon, x):(\varepsilon, x) \in] 0, \eta[\times K\} \subset K^{\prime} .
$$

Sejam $V$ aberto de $\mathbb{R}^{p}$ tal que $K^{\prime} \subset V \subset \bar{V} \subset \subset \Omega^{\prime}$ e seja $r$ a distância de $K^{\prime}$ a $\Omega^{\prime} \backslash V$. Então

$$
K^{\prime} \subset \cup_{y \in K^{\prime}} B_{\frac{\mathrm{r}}{8}}(y) \subset \cup_{y \in K^{\prime}} B_{\frac{\mathrm{r}}{4}}(y) \subset \bar{V} \subset \Omega^{\prime},
$$

e assim existem $y_{1}, \ldots, y_{s}$ em $K^{\prime}$ tais que

$$
K^{\prime} \subset \cup_{i=1}^{s} B_{\frac{r}{8}}\left(y_{i}\right) \subset \cup_{i=1}^{s} \overline{B_{\frac{r}{4}}\left(y_{i}\right)} \subset \subset \Omega^{\prime} .
$$

Usando que $\hat{f}-\hat{g} \in \mathcal{N}\left[\Omega ; \mathbb{R}^{p}\right]$ existem $\left.\eta_{1} \in\right] 0, \eta[$ e $c>0$ tais que

$$
\left.\left|\widehat{f}_{i}(\varepsilon, x)-\widehat{g}_{i}(\varepsilon, x)\right| \leq c \varepsilon \text {, para todo }(\varepsilon, x) \in\right] 0, \eta_{1}[\times K \text { e } 1 \leq i \leq p .
$$

Seja $\eta_{2}=\min \left\{\eta_{1}, \frac{r}{8 c p}\right\}$. Então se $\left.\varepsilon \in\right] 0, \eta_{2}[$ e $x \in K$ temos, por (1) e (2), que existe $j \in\{1,2, \ldots, s\}$ tal que $\hat{f}(\varepsilon, x) \in B_{\frac{r}{8}}\left(y_{j}\right)$ e assim, por (3), concluímos que

$$
\left\|\hat{g}(\varepsilon, x)-y_{j}\right\| \leq\|\hat{g}(\varepsilon, x)-\widehat{f}(\varepsilon, x)\|+\left\|\widehat{f}(\varepsilon, x)-y_{j}\right\|<c p \varepsilon+\frac{r}{8} \leq \frac{r}{8}+\frac{r}{8}=\frac{r}{4} .
$$

Portanto

$$
\{\hat{g}(\varepsilon, x):(\varepsilon, x) \in] 0, \eta_{2}[\times K\} \subset \cup_{i=1}^{s} \overline{B_{\frac{r}{4}}\left(y_{i}\right)} \subset \subset \Omega^{\prime} . / /
$$

No próximo resultado mostramos que $\mathcal{G}\left(., \mathbb{R}^{n}\right)$ é um pré-feixe completo de espaços vetoriais sobre $\mathbb{R}^{n}$. 
1.1.20 Proposição. Sejam $\Omega$ um aberto de $\mathbb{R}^{n}$ que é reunião de uma família $\left(\Omega_{i}\right)_{i \in I}$ de abertos não vazios de $\mathbb{R}^{n}$ e $p \in \mathbb{N}^{*}$. As seguintes asserções são verdadeiras:

(I) se $f$ e g são funções de $\mathcal{G}\left(\Omega ; \mathbb{R}^{p}\right)$ tais que $\left.f\right|_{\Omega_{i}}=\left.g\right|_{\Omega_{i}}$, para todo $i \in I$, então $f=g$;

(II) se $\left(f_{i}\right)_{i \in I}$ é uma família tal que $f_{i} \in \mathcal{G}\left(\Omega_{i} ; \mathbb{R}^{p}\right)$ para todo $i \in I$, e $\left.f_{i}\right|_{\Omega_{i} \cap \Omega_{j}}=$ $\left.f_{j}\right|_{\Omega_{i} \cap \Omega}$, para tocio $(i, j) \in I \times I \operatorname{com} \Omega_{i} \cap \Omega_{j} \neq \emptyset$, então e.riste $f \in \mathcal{G}\left(\Omega ; \mathbb{R}^{p}\right)$ com $\left.f\right|_{\Omega_{i}}=f_{i}$, para todo $i \in I$;

(III) se $\Omega^{\prime}$ é um aberto de $\mathbb{R}^{p}, f \in \mathcal{G}\left(\Omega ; \mathbb{R}^{p}\right)$ e $\left(f_{i}\right)_{i \in I}$ é uma família tal que $f_{i} \in$ $\mathcal{G}_{*}\left(\Omega_{i} ; \Omega^{\prime}\right)$ e $\left.f\right|_{\Omega_{i}}=f_{i}$, para todo $i \in I$, então $f \in \mathcal{G}_{*}\left(\Omega ; \Omega^{\prime}\right)$.

Demonstração. Com demonstração análoga à encontrada em [2.3.2] de [2] temos que (I) e (II) são verdadeiras. Provaremos (III).

Sejam $K \subset \subset \Omega=\cup_{i \in I} \Omega_{i}$ e $\hat{f}$ um representante de $f$. Provaremos que existem $K^{\prime} \subset \subset \Omega^{\prime}$ e $\left.\left.\eta \in\right] 0,1\right]$ tais que

$$
\{\widehat{f}(\varepsilon, x):(\varepsilon, x) \in] 0, \eta[\times K\} \subset K^{\prime} .
$$

Para $i \in I$ e $x \in \Omega_{i}$ seja $r_{i}(x)>0$ tal que $\overline{B_{r_{i}(x)}(x)} \subset \Omega_{i}$.

Como $K$ é um conjunto compacto de $\mathbb{R}^{n}$ e $K \subset \cup_{i \in I} \cup_{x \in \Omega_{i}} B_{r_{i}(x)}(x)$, existem $i_{1}, \ldots, i_{s}$ em $I$ e $x_{i_{1}}, \ldots, x_{i_{s}}$ em $\Omega$ tais que

$$
K \subset \cup_{j=1}^{s} B_{r_{i_{j}}\left(x_{i_{j}}\right)}\left(x_{i_{j}}\right) \text { e } x_{i_{j}} \in \Omega_{i_{j}} \text {, para todo } 1 \leq j \leq s .
$$

Para $1 \leq j \leq s$ seja $L_{j}=\overline{B_{r_{i j}\left(x_{i j}\right)}\left(x_{i_{j}}\right)} \subset \Omega_{i_{j}}$. Então $K \subset \cup_{j=1}^{s} L_{j}$ e $L_{j} \subset \subset$ $\Omega_{i,}$, para todo $1 \leq j \leq s$.

Fixemos $j \in\{1, \ldots, s\}$. Como $L_{j} \subset \subset \Omega_{i_{j}},\left.f\right|_{\Omega_{i j}}=f_{i_{j}}$ e $f_{i_{j}} \in \mathcal{G}_{*}\left(\Omega_{i_{j}} ; \Omega^{\prime}\right)$ temos que existem $R_{j} \subset \subset \Omega^{\prime}$ e $\left.\left.\eta_{j} \in\right] 0,1\right]$ tais que

$$
\{\widehat{f}(\varepsilon, x):(\varepsilon, x) \in] 0, \eta_{j}\left[\times L_{j}\right\} \subset R_{j} \subset \subset \Omega^{\prime} .
$$

Portanto $\cup_{j=1}^{s} R_{j} \subset \subset \Omega^{\prime}$ e para $\bar{\eta}=\min \left\{\eta_{1}, \ldots, \eta_{s}\right\}$ temos que

$$
\{\hat{f}(\varepsilon, x): x \in K \text { e } \varepsilon \in] 0, \bar{\eta}[\} \subset \cup_{j=1}^{s}\left\{\hat{f}(\varepsilon, x): x \in L_{j} \text { e } \varepsilon \in\right] 0, \bar{\eta}[\} \subset \cup_{j=1}^{s} R_{j} \subset \subset \Omega^{\prime}
$$


e $\operatorname{assim}(1)$ é verdadeiro. //

Em [2] encontramos uma definição de composta para aplicações generalizadas na teoria geral. De modo análogo obtemos uma definição para a álgebra que estamos trabalhando. Procurando facilitar a leitura iremos descrever os passos necessários para obter 1.1.23.

1.1.21 Lema. Sejam $\Omega$ um aberto de $\mathbb{R}^{n}, \Omega^{\prime}$ uri aberto de $\mathbb{R}^{p}, f \in \mathcal{G}_{*}\left(\Omega ; \Omega^{\prime}\right), g \in$ $\mathcal{G}\left(\Omega^{\prime} ; \mathbb{R}^{s}\right), \hat{f}$ um representante de $f, \hat{g}$ um representante de $g, \mathcal{K}=\left(K_{j}\right)_{j \in N}$ uma seqüência exaustiva de compactos para $\Omega$, isto é,

$$
\cup_{j \in N} K_{j}=\Omega, \quad K_{j} \subset K_{j+1}^{\circ} \text { e } K_{j} \subset \subset \Omega, \text { para todo } j \in \mathbb{N}
$$

e seja $\left(\eta_{j}\right)_{j \in N}$ uma seqüência em ]0,1] satisfazendo :

(i) $\eta_{j}>\eta_{j+1}$, para todo $j \in \mathbb{N}$;

(ii) $\overline{\{\hat{f}(\varepsilon, x):(\varepsilon, x) \in] 0, \eta_{j}\left[\times K_{j}\right\}} \subset \subset \Omega^{\prime}$, para todo $j \in \mathbb{N}$.

Sejam $j \in \mathbb{N}$ e $\hat{h}_{j}$ a aplicação definida em $\left.] 0,1\right] \times \stackrel{\circ}{K}_{j}$ por

$$
\widehat{h}_{j}(\varepsilon, x)=\left\{\begin{array}{ll}
\hat{g}(\varepsilon, \hat{f}(\varepsilon, x)) & \text {, se } \varepsilon \in] 0, \eta_{j}[ \\
\hat{g}\left(\varepsilon, \hat{f}\left(\frac{\eta_{j}}{2}, x\right)\right) & \text {, se } \varepsilon \in\left[\eta_{j}, 1\right]
\end{array} .\right.
$$

Então

(I) $\widehat{h}_{j} \in \mathcal{E}_{M}\left[\stackrel{\circ}{K}_{j} ; \mathbb{R}^{s}\right]$, para todo $j \in \mathbb{N}$;

(II) existe $\Theta_{\mathcal{K}, \hat{f}, \hat{g}} \in \mathcal{E}_{M}\left[\Omega ; \mathbb{R}^{s}\right]$ tal que $\left.\Theta_{\mathcal{K}, \hat{f}, \hat{g}}\right|_{] 0,1] \times K_{j}}-\widehat{h}_{j} \in \mathcal{N}\left[\stackrel{\circ}{K}_{j} ; \mathbb{R}^{s}\right]$, para todo $j \in \mathbb{N}$.

(Convém observar que de 1.1.18.i garantimos a existência da seqüência $\left(\eta_{j}\right)_{j \in N}$ satisfazendo 1.1.21.i e 1.1.21.ii.)

Demonstração. O item (I) segue de [3.1.9.a] de [14]. A asserção (II) segue de 1.1.20.II fazendo $\Omega_{i}=\stackrel{\circ}{K_{i}}$ e $f_{i}$ a classe de $\widehat{h}_{i}$ em $\mathcal{G}\left(\stackrel{\circ}{K}_{i} ; \mathbb{R}^{s}\right)$. // 
1.1.22 Lema. Sejam $\Omega$ um aberto de $\mathbb{R}^{n}, \Omega^{\prime}$ um aberto de $\mathbb{R}^{p}, f \in \mathcal{G}_{*}\left(\Omega ; \Omega^{\prime}\right), g \in$ $\mathcal{G}\left(\Omega^{\prime} ; \mathbb{R}^{s}\right), \hat{f}$ um representante de $f$ e $\hat{g}$ um representante de $g$. Com as notações do lema anterior, as seguintes afirmações são verdadeiras :

(I) se $\mathcal{K}=\left(K_{j}\right)_{j \in N}$ é uma seqüência exaustiva de compactos para $\Omega, \widehat{f}_{1}$ é um representante de $f$ e $\widehat{g}_{1}$ é um representante de $g$, então $\Theta_{\mathcal{K}, \widehat{f}, \hat{g}}-\Theta_{\mathcal{K}, \widehat{f}_{1}, \widehat{g}_{1}} \in \mathcal{N}\left[\Omega ; \mathbb{R}^{s}\right]$;

(II) se $\mathcal{K}=\left(K_{j}\right)_{j \in N}$ e $\mathcal{H}=\left(H_{j}\right)_{j \in N}$ sãs seqüências exaustivas de compactos para $\Omega$, $\widehat{f}_{1}$ um representante de $f$ e $\widehat{g}_{1}$ um representante de $g$, então

$$
\Theta_{\mathcal{K}, \widehat{f}, \widehat{g}}-\Theta_{\mathcal{H}, \widehat{f}_{1}, g_{1}} \in \mathcal{N}\left[\Omega ; \mathbb{R}^{s}\right]
$$

(III) se $\mathcal{K}=\left(K_{j}\right)_{j \in N}$ é uma seqüência exaustiva de compactos para $\Omega$ e existem $\tau \in] 0,1]$ e $\hat{f}_{1}$ um representante de $f \operatorname{com} \hat{f}_{1}(] 0, \tau[\times \Omega) \subset \Omega^{\prime}$, então a aplicação definida em $] 0,1] \times \Omega$ por

$$
\hat{l}(\varepsilon, x)=\left\{\begin{array}{ll}
\hat{g}\left(\varepsilon, \hat{f}_{1}(\varepsilon, x)\right) & , \text { se } \varepsilon \in] 0, \tau[ \\
\hat{g}\left(\varepsilon, \hat{f}_{1}\left(\frac{\tau}{2}, x\right)\right) & , \text { se } \varepsilon \in[\tau, 1]
\end{array},\right.
$$

pertence a $\mathcal{E}_{M}\left[\Omega ; \mathbb{R}^{s}\right]$ e $\Theta_{\mathcal{K}, \widehat{f}, \widehat{g}}-\hat{l} \in \mathcal{N}\left[\Omega ; \mathbb{R}^{s}\right]$.

Demonstração. Como estamos usando as notações do lema anterior, existem $\left(\eta_{j}\right)_{j \in N}$ e $\left(\bar{\eta}_{j}\right)_{j \in N}$ tais que $1 \geq \eta_{j}>\eta_{j+1}>0,1 \geq \bar{\eta}_{j}>\bar{\eta}_{j+1}>0$,

$$
\begin{aligned}
& \overline{\{\hat{f}(\varepsilon, x):(\varepsilon, x) \in] 0, \eta_{j}\left[\times K_{j}\right\}} \subset \subset \Omega^{\prime}, \text { para todo } j \in \mathbb{N}, \\
& \overline{\left\{\hat{f}_{1}(\varepsilon, x):(\varepsilon, x) \in\right] 0, \bar{\eta}_{j}\left[\times K_{j}\right\}} \subset \subset \Omega^{\prime}, \text { para todo } j \in \mathbb{N},
\end{aligned}
$$

e existem $\widehat{h}_{j}$ e $\hat{l}_{j}$ aplicações moderadas definidas em $\left.] 0,1\right] \times \stackrel{0}{K}_{j}$ por

$$
\begin{gathered}
\hat{h}_{j}(\varepsilon, x)=\left\{\begin{array}{ll}
\hat{g}(\varepsilon, \hat{f}(\varepsilon, x)) & , \text { se } \varepsilon \in] 0, \eta_{j}[ \\
\hat{g}\left(\varepsilon, \hat{f}\left(\frac{\eta_{j}}{2}, x\right)\right) & , \text { se } \varepsilon \in\left[\eta_{j}, 1\right]
\end{array} ;\right. \\
\hat{l}_{j}(\varepsilon, x)=\left\{\begin{array}{ll}
\widehat{g}_{1}\left(\varepsilon, \widehat{f}_{1}(\varepsilon, x)\right) & , \text { se } \varepsilon \in] 0, \bar{\eta}_{j}[ \\
\widehat{g}_{1}\left(\varepsilon, \widehat{f}_{1}\left(\frac{\bar{\eta}_{j}}{2}, x\right)\right) & , \text { se } \varepsilon \in\left[\bar{\eta}_{j}, 1\right]
\end{array},\right.
\end{gathered}
$$


e tais que $\left.\Theta_{\mathcal{K}, \hat{f}, \hat{g}}\right|_{\mathrm{j} 0,1] \times K^{\circ}}, \hat{h}_{j} \in \mathcal{N}\left[\stackrel{\circ}{K}_{j} ; \mathbb{R}^{s}\right]$ e $\left.\Theta_{\mathcal{K}, \hat{f}_{1}, \hat{g}_{1}}\right|_{j 0,1] \times K_{j}}-\hat{l}_{j} \in \mathcal{N}\left[\stackrel{\circ}{K}_{j} ; \mathbb{R}^{s}\right]$, para todo $j \in \mathbb{N}$.

Sejam $K \subset \subset \Omega$ e $\alpha \in \mathbb{N}^{n}$. Provaremos que existem $\left.\left.\eta \in\right] 0,1\right], c>0$ e q $\in \mathbb{N}$ tais que

$$
\left.\left\|\partial^{\alpha} \Theta_{\mathcal{K} \hat{j}, \widehat{g}}(\varepsilon, x)-\partial^{\alpha} \Theta_{\mathcal{K}, \widehat{\jmath}_{1}, \widehat{g}_{1}}(\varepsilon, x)\right\| \leq c \varepsilon^{q}, \text { para todo }(\varepsilon, x) \in\right] 0, \eta[\times K .
$$

Seja $j \in \mathbb{N}$ tal que $K \subset \stackrel{\circ}{K}$.

Usando que $\left.\left(\widehat{f}-\widehat{f}_{1}\right)\right|_{\mathrm{j}_{0,1] \times K}, 0} \in \mathcal{N}\left[\stackrel{\circ}{K}_{j} ; \mathbb{R}^{p}\right]$ e que $\hat{g}-\widehat{g}_{1} \in \mathcal{N}\left[\Omega^{\prime} ; \mathbb{R}^{s}\right]$ temos, por [3.1.9.d] de [14], que $\hat{h}_{j}-\hat{l}_{j} \in \mathcal{N}\left[\dot{\circ}_{j} ; \mathbb{R}^{s}\right]$, e portanto

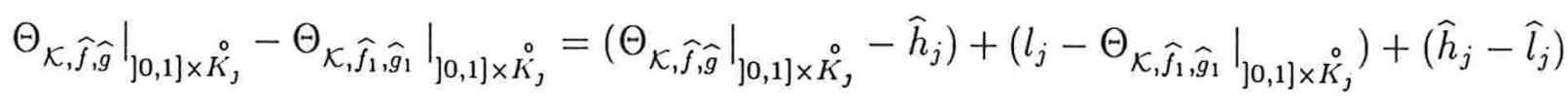
pertence a $\mathcal{N}\left[\stackrel{\circ}{K}_{j} ; \mathbb{R}^{s}\right]$.

Como $K \subset \subset \stackrel{\circ}{K_{j}}$ e $\Theta_{\mathcal{K}, \widehat{f}, \widehat{g}}-\Theta_{\mathcal{K}, \widehat{f}_{1}, \widehat{g}_{1}} \in \mathcal{N}\left[\stackrel{\circ}{K}_{j} ; \mathbb{R}^{s}\right]$ é fácil verificar que (1) é verdadeira, e assim concluímos (I).

Provaremos a seguir (II).

Como

$$
\Theta_{\mathcal{K}, \widehat{f}, \hat{g}}-\Theta_{\mathcal{H}, \widehat{f}_{1}, \widehat{g}_{1}}=\Theta_{\mathcal{K}, \hat{f}, \widehat{g}}-\Theta_{\mathcal{K}, \widehat{f}_{1}, \widehat{g}_{1}}+\Theta_{\mathcal{K}, \widehat{f}_{1}, \hat{g}_{1}}-\Theta_{\mathcal{H}, \widehat{f}_{1}, \widehat{g}_{1}}
$$

e vale (I), basta verificar que $\Theta_{\mathcal{K},{\widehat{f_{1}}}_{1}, \hat{g}_{1}}-\Theta_{\mathcal{H}, \widehat{f}_{1}, \widehat{g}_{1}} \in \mathcal{N}\left[\Omega ; \mathbb{R}^{s}\right]$.

Como estamos usando as notações do lema anterior existe $\left(\nu_{j}\right)_{j \in N}$ tal que $1 \geq \nu_{j}>$ $\nu_{j+1}>0 \mathrm{e}$

$$
\overline{\left\{\hat{f}_{1}(\varepsilon, x):(\varepsilon, x) \in\right] 0, \nu_{j}\left[\times H_{j}\right\}} \subset \subset \Omega^{\prime}, \text { para todo } j \in \mathbb{N}
$$

e existe $\hat{s}_{j}$ aplicação moderada definida em $\left.] 0,1\right] \times \stackrel{\circ}{H}_{j}$ por

$$
\widehat{s}_{j}(\varepsilon, x)= \begin{cases}\widehat{g}_{1}\left(\varepsilon, \hat{f}_{1}(\varepsilon, x)\right) & , \text { se } \varepsilon \in] 0, \nu_{j}[ \\ \widehat{g}_{1}\left(\varepsilon, \hat{f}_{1}\left(\frac{\nu_{j}}{2}, x\right)\right) & , \text { se } \varepsilon \in\left[\nu_{j}, 1\right]\end{cases}
$$

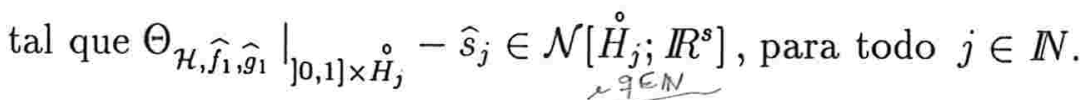

Sejam $K \subset \subset \Omega$ e $\alpha \in \mathbb{N}^{n}$. Provaremos que existem $\left.\left.\nu \in\right] 0,1\right], c>0$ e tais que

$$
\left.\left\|\partial^{\alpha} \Theta_{\mathcal{K}, \widehat{f_{1}}, \hat{g}_{1}}(\varepsilon, x)-\partial^{\alpha} \Theta_{\mathcal{H}, \widehat{\hat{f}_{1}}, \widehat{g}_{1}}(\varepsilon, x)\right\| \leq c \varepsilon^{q} \text {, para todo }(\varepsilon, x) \in\right] 0, \nu[\times K .
$$


Como $\mathcal{K}$ e $\mathcal{H}$ são seqüências exaustivas de compactos para $\Omega$, existe $j \in \mathbb{N}$ tal que $K \subset \stackrel{\circ}{K}_{j} \cap \stackrel{\circ}{H}_{j}$. Tomando $\bar{\eta}=\min \left\{\bar{\eta}_{j}, \nu_{j}\right\}$ temos que $\hat{l}_{j}=\widehat{s}_{j}$ em $] 0, \bar{\eta}\left[\times\left(\stackrel{\circ}{K}_{j} \cap \stackrel{\circ}{H}_{j}\right)\right.$, e portanto

$$
\Theta_{\mathcal{K}, \widehat{f}_{1}, \widehat{g}_{1}}-\Theta_{\mathcal{H}, \widehat{f}_{1}, \widehat{g}_{1}}=\left(\Theta_{\mathcal{K}, \widehat{f}_{1}, \widehat{g}_{1}}-\widehat{l}_{j}\right)+\left(\widehat{s}_{j}-\Theta_{\mathcal{H} \widehat{f}_{1}, \widehat{g}_{1}}\right)
$$

em $] 0, \bar{\eta}\left[\times\left(\stackrel{\circ}{K}_{j} \cap \stackrel{\circ}{H}_{j}\right)\right.$.

Usando a igualdade acima e que

$$
\left.\Theta_{\mathcal{K}, \widehat{f}_{1}, \widehat{g}_{1}}\right|_{\mathrm{j} 0,1] \times \stackrel{\circ}{K}_{j}}-\hat{l}_{j} \in \mathcal{N}\left[\stackrel{\circ}{K}_{j} ; \mathbb{R}^{s}\right] \text { e }\left.\Theta_{\mathcal{H}, \widehat{f}_{1}, \widehat{g}_{1}}\right|_{\mathrm{j} 0,1] \times \stackrel{\circ}{H}_{j}}-\widehat{s}_{j} \in \mathcal{N}\left[\stackrel{\circ}{H}_{j} ; \mathbb{R}^{s}\right]
$$

é fácil verificar que $\left.\left(\Theta_{\mathcal{K}, \widehat{f}_{1}, \widehat{g}_{1}}-\Theta_{\mathcal{H}, \widehat{f}_{1}, \widehat{g}_{1}}\right)\right|_{\mathrm{j} 0,1] \times\left(\stackrel{\circ}{K}_{j} \cap \stackrel{\circ}{H}_{j}\right)} \in \mathcal{N}\left[\stackrel{\circ}{K}_{j} \cap \stackrel{\circ}{H}_{j} ; \mathbb{R}^{s}\right]$ e portanto (2) é verdadeira, e assim obtemos (II).

Finalmente provaremos (III).

Usando [3.1.9.a] de [14] temos que $\hat{l} \in \mathcal{E}_{M}\left[\Omega ; \mathbb{R}^{s}\right]$.

Sejam $K \subset \subset \Omega$ e $\alpha \in \mathbb{N}^{n} \%$ Provaremos que existem $\left.\left.\eta \in\right] 0,1\right], c>0$ e $q \in \mathbb{R} \in$ tais que

$$
\left.\left|\partial^{\alpha} \Theta_{\mathcal{K}, \hat{f}, \hat{g}}(\varepsilon, x)-\partial^{\alpha} \hat{l}(\varepsilon, x)\right| \leq c \varepsilon^{q}, \text { para todo }(\varepsilon, x) \in\right] 0, \eta[\times K
$$

Seja $j \in \mathbb{I}$ tal que $K \subset \stackrel{\circ}{K}_{j}$.

Como $\left.\Theta_{\mathcal{K}, \hat{f}, \hat{g}}\right|_{\mathrm{j} 0,1] \times \stackrel{\circ}{K}_{j}}-\hat{h}_{j} \in \mathcal{N}\left[\stackrel{\circ}{K}_{j} ; \mathbb{R}^{s}\right]$ e $\hat{h}_{j}-\left.\hat{l}\right|_{\mathrm{j} 0,1] \times \stackrel{\circ}{K}_{j}} \in \mathcal{N}\left[\stackrel{\circ}{K}_{j} ; \mathbb{R}^{s}\right]$ ([3.1.9.c] de [14]), é fácil verificar que (3) é verdadeira, e assim concluímos (III). //

Usando o lema acima podemos introduzir a seguinte definição, que é análoga à encontrada em [2].

1.1.23 Definição. Sejam $\Omega$ um aberto de $\mathbb{R}^{n}, \Omega^{\prime}$ um aberto de $\mathbb{R}^{p}, f \in \mathcal{G}_{*}\left(\Omega ; \Omega^{\prime}\right)$ e $g \in \mathcal{G}\left(\Omega^{\prime} ; \mathbb{R}^{s}\right)$. Denotamos por $g \circ f$ a classe da aplicação moderada $\Theta_{\mathcal{K}, \hat{f}, \hat{g}}$, definida em 1.1.21.II, em $\mathcal{G}\left(\Omega ; \mathbb{R}^{s}\right)$, sendo $\mathcal{K}=\left(K_{j}\right)_{j \in N}$ uma seqüencia exaustiva de compactos para $\Omega, \hat{f}$ um representante de $f$ e $\hat{g}$ um representante de $g$.

A seguir apresentamos alguns resultados que envolvem a noção de função composta e que serão úteis neste trabalho. 
1.1.24 Proposição. Sejam $\Omega$ um aberto de $\mathbb{R}^{n}, \Omega^{\prime}$ um aberto de $\mathbb{R}^{p}$ e $\alpha \in \mathbb{N}^{n}$. Se V é um aberto de $\mathbb{R}^{n}$ com $V \subset \Omega, f \in \mathcal{G}_{*}\left(\Omega ; \Omega^{\prime}\right)$ e $g \in \mathcal{G}\left(\Omega^{\prime} ; \mathbb{R}^{s}\right)$, então

$$
\left.f\right|_{V} \in \mathcal{G}_{*}\left(V ; \Omega^{\prime}\right),\left.\quad(g \circ f)\right|_{V}=\left.g \circ\left(\left.f\right|_{V}\right) \quad e \quad\left(\partial^{\alpha} f\right)\right|_{V}=\partial^{\alpha}\left(\left.f\right|_{V}\right)
$$

Demonstração. Usando a definição de $\left.f\right|_{V}$ e que $f \in \mathcal{G}_{*}\left(\Omega ; \Omega^{\prime}\right)$ é fácil verificar que $\left.f\right|_{V} \in$ $\mathcal{G}_{*}\left(V ; \Omega^{\prime}\right)$. Provaremos que $\left.(g \circ f)\right|_{V}=g \circ\left(\left.f\right|_{V}\right)$.

Sejam $\mathcal{K}=\left(K_{j}\right)_{j \in N}$ uma seqüência exaustiva de compactos para $V, \hat{f}$ um representante de $f$ e $\hat{g}$ um representante de $g$.

Como $\left.f\right|_{V} \in \mathcal{G}_{*}\left(V ; \Omega^{\prime}\right)$ e $\hat{\varphi}=\left.\widehat{f}\right|_{j 0,1] \times V}$ é um representante de $\left.f\right|_{V}$, existe uma seqüência $\left(\eta_{j}\right)_{j \in N}$ tal que $1 \geq \eta_{j}>\eta_{j+1}>0$ e

$$
\overline{\{\hat{\varphi}(\varepsilon, x):(\varepsilon, x) \in] 0, \eta_{j}\left[\times K_{j}\right\}} \subset \subset \Omega^{\prime} \text {, para todo } j \in \mathbb{N} \text {. }
$$

Para $j \in \mathbb{N}$, seja $\hat{h}_{j}$ a aplicação definida em $\left.] 0,1\right] \times \stackrel{\circ}{K}_{j}$ por

$$
\hat{h}_{j}(\varepsilon, x)= \begin{cases}\hat{g}(\varepsilon, \hat{\varphi}(\varepsilon, x)) & , \text { se } \varepsilon \in] 0, \eta_{j}[ \\ \hat{g}\left(\varepsilon, \hat{\varphi}\left(\frac{\eta_{j}}{2}, x\right)\right) & , \text { se } \varepsilon \in\left[\eta_{j}, 1\right]\end{cases}
$$

e seja $l=g \circ\left(\left.f\right|_{V}\right)$.

Por 1.1.21.II temos que $\widehat{h}_{j}$ é um representante de $\left.l\right|_{K_{j}^{\circ}}$ e de $\left.(g \circ f)\right|_{K_{j}^{\circ}}$, para todo $j \in \mathbb{N}$, e assim, por 1.1.20.I, concluímos que $g \circ\left(\left.f\right|_{V}\right)=l=\left.(g \circ f)\right|_{V}$.

Finalmente, usando que $\left.\widehat{f}\right|_{j 0,1] \times V}$ é um representante de $\left.f\right|_{V}$, obtemos que $\left.\left(\partial^{\alpha} f\right)\right|_{V}=$ $\partial^{\alpha}\left(\left.f\right|_{V}\right) \cdot / /$

1.1.25 Proposição. Sejam $\Omega^{\prime}$ um aberto de $\mathbb{R}^{n+1}, \Omega^{\prime \prime}$ aberto de $\mathbb{R}^{p}, t_{o} \in \mathbb{R}$ com $\Omega=$ $\left\{z \in \mathbb{R}^{n}:\left(t_{o}, z\right) \in \Omega^{\prime}\right\} \neq \emptyset, V$ aberto de $\mathbb{R}^{n} \operatorname{com} V \subset \Omega, f \in \mathcal{G}_{*}\left(\Omega^{\prime} ; \Omega^{\prime \prime}\right), g \in \mathcal{G}\left(\Omega^{\prime \prime} ; \mathbb{R}^{s}\right)$, $\alpha=\left(\alpha_{0}, \alpha_{1}, \ldots, \alpha_{n}\right) \in \mathbb{N}^{n+1} \operatorname{com} \alpha_{0}=0$ e $\beta=\left(\alpha_{1}, \ldots, \alpha_{n}\right)$. Então

(I) $\left.f\right|_{\left\{t_{0}\right\} \times V} \in \mathcal{G}_{*}\left(V ; \Omega^{\prime \prime}\right)$;

(II) $\left.(g \circ f)\right|_{\left\{t_{o}\right\} \times V}=g \circ\left(\left.f\right|_{\left\{t_{o}\right\} \times V}\right)$ em $\mathcal{G}\left(V ; \mathbb{R}^{s}\right)$;

(III) $\left.\left(\partial^{\alpha} f\right)\right|_{\left\{t_{o}\right\} \times V}=\partial^{\beta}\left(\left.f\right|_{\left\{t_{o}\right\} \times V}\right)$ em $\mathcal{G}\left(V ; \mathbb{R}^{p}\right)$. 
Demonstração. Como $f \in \mathcal{G}_{*}\left(\Omega^{\prime} ; \Omega^{\prime \prime}\right)$ e dado qualquer $K \subset \subset V$ temos que $\left\{t_{o}\right\} \times K \subset \subset \Omega^{\prime}$, é fácil verificar (I).

Para (II) tomemos $\mathcal{K}=\left(K_{j}\right)_{j \in N}$ uma seqüência exaustiva de compactos para $V$ e $\mathcal{K}^{\prime}=\left(K_{j}^{\prime}\right)_{j \in N}$ uma seqüência exaustiva de compactos para $\Omega^{\prime}$.

Como $\left(\left\{t_{o}\right\} \times K_{j}\right)_{j \in N}$ é uma seqüência de compactos em $\Omega^{\prime}$ e $\left(K_{j}^{\prime}\right)_{j \in N}$ é uma seqüência exaustiva de compactos para $\Omega^{\prime}$ existe uma seqüência $\left(\nu_{j}\right)_{j \in N}$ de números naturais tal que

$$
\nu_{j}<\nu_{j+1} \quad \text { e } \quad\left\{t_{o}\right\} \times K_{j} \subset \stackrel{\circ}{K_{\nu_{j}}^{\prime}} .
$$

Usando que $f \in \mathcal{G}_{*}\left(\Omega^{\prime} ; \Omega^{\prime \prime}\right)$ existe $\left(\eta_{j}\right)_{j \in N}$ tal que $1 \geq \eta_{j}>\eta_{j+1}>0$ e

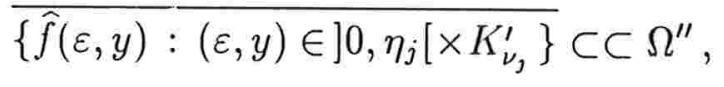

e assim podemos definir em $] 0,1] \times \stackrel{0}{K_{\nu_{j}}^{\prime}}$ a aplicação

$$
\widehat{h}_{j}(\varepsilon, x)=\left\{\begin{array}{ll}
\hat{g}(\varepsilon, \hat{f}(\varepsilon, x)) & , \text { se } \varepsilon \in] 0, \eta_{j}[ \\
\widehat{g}\left(\varepsilon, \widehat{f}\left(\frac{\eta_{j}}{2}, x\right)\right) & , \text { se } \varepsilon \in\left[\eta_{j}, 1\right]
\end{array},\right.
$$

para todo $j \in \mathbb{N}$.

Fixemos $j \in \mathbb{N}$.

Seja $s=\left.(g \circ f)\right|_{\left\{t_{o}\right\} \times V}$. Então por 1.1.21.II temos que $\widehat{h}_{j}$ é um representante de $\left.(g \circ f)\right|_{K_{\nu_{j}}^{\circ}} ^{\circ}$.

Seja $W_{j}=\left\{y \in \mathbb{R}^{n}:\left(t_{o}, y\right) \in \stackrel{\circ}{K_{\nu_{j}}^{\prime}}\right\}$. Como $\left\{t_{o}\right\} \times K_{j} \subset \stackrel{\circ}{K_{\nu_{j}}^{\prime}}$ temos que $W_{j} \neq \emptyset \mathrm{e}$ $\stackrel{\circ}{K_{j}} \subset W_{j}$. Então $\left.\widehat{h}_{j}\right|_{\mathrm{j} 0,1] \times\left\{t_{0}\right\} \times \stackrel{\circ}{K_{j}}}$ é um representante de $\left.s\right|_{K_{j}^{\circ}}$.

Como $V \subset\left\{y \in \mathbb{R}^{n}:\left(t_{o}, y\right) \in \Omega^{\prime}\right\}$ temos que $\left.\hat{f}\right|_{j 0,1] \times\left\{t_{o}\right\} \times V}$ é um representante de $f \mid\left\{t_{o}\right\} \times V$.

Sejam $r=\left.f\right|_{\left\{t_{o}\right\} \times V}$ e $l=g \circ r$. Por 1.1 .24 sabemos que $\left.l\right|_{K_{j}}=\left.(g \circ r)\right|_{K_{j}}=g \circ\left(\left.r\right|_{K_{j}}\right)$, e como $\left.\widehat{f}\right|_{\mathrm{j} 0,1] \times\left\{t_{0}\right\} \times K_{j}}$ é um representante de $\left.r\right|_{K_{j}^{\circ}}$ temos, por 1.1.22.III, que $\left.\widehat{h}_{j}\right|_{\mathrm{J} 0,1] \times\left\{t_{0}\right\} \times K_{j}}$ é um representante de $\left.l\right|_{K_{j}^{\circ}}$, para todo $j \in \mathbb{N}$.

Portanto $\left.s\right|_{K_{j}}=\left.l\right|_{K_{j}}$ para todo $j \in \mathbb{N}$, e assim, por 1.1.20.I, concluímos que

$$
\left.(g \circ f)\right|_{\left\{t_{o}\right\} \times V}=s=l=g \circ\left(\left.f\right|_{\left\{t_{o}\right\} \times V}\right),
$$


o que prova (II).

Finalmente tomemos $\alpha=\left(\alpha_{0}, \alpha_{1}, \ldots, \alpha_{n}\right) \in \mathbb{N}^{n+1} \operatorname{com} \alpha_{0}=0, \beta=\left(\alpha_{1}, \ldots, \alpha_{n}\right) \in \mathbb{N}^{n}$ e $\hat{f}$ um representante de $f$.

Como $\left.\hat{f}\right|_{\jmath 0,1] \times\left\{t_{o}\right\} \times V}$ é um representante de $\left.f\right|_{\left\{t_{o}\right\} \times V}$ e $\partial^{\alpha} \widehat{f}\left(\varepsilon, t_{o},.\right)=\partial^{\beta}\left(\widehat{f}\left(\varepsilon, t_{o},.\right)\right)$ temos que $\left.\left(\partial^{\alpha} f\right)\right|_{\left\{t_{o}\right\} \times V}=\partial^{\beta}\left(\left.f\right|_{\left\{t_{o}\right\} \times V}\right)$, e portanto (III) é verdadeira. //

1.1.26 Proposição. Sejam $\Omega$ um aberto de $\mathbb{R}^{n}$ e $\Omega^{\prime}$ um aberto de $\mathbb{R}^{p}$. Se $f \in$ $\mathcal{G}_{*}\left(\Omega ; \Omega^{\prime}\right), g \in \mathcal{G}\left(\Omega^{\prime} ; \mathbb{R}^{s}\right)$ e $h \in \mathcal{G}\left(\Omega^{\prime} ; \mathbb{R}^{r}\right)$, então $(g, h) \circ f=(g \circ f, h \circ f)$, onde o símbolo $(g, h)$ foi definido em 1.1.10.

Demonstração. Seja $\mathcal{K}=\left(K_{j}\right)_{j \in N}$ uma seqüência exaustiva de compactos para $\Omega$.

Provaremos que $\left.((g, h) \circ f)\right|_{K_{j}} ^{\circ}=\left.(g \circ f, h \circ f)\right|_{K_{j}}$ para todo $j \in \mathbb{N}$, e assim, por 1.1.20.I, teremos o resultado desejado.

Sejam $\hat{g}, \hat{h}$ e $\hat{f}$ representantes, respectivamente, de $g, h$ e $f$.

Como $f \in \mathcal{G}_{*}\left(\Omega ; \Omega^{\prime}\right)$ existe $\left.\left.\eta_{j} \in\right] 0,1\right]$ tal que

$$
\overline{\{\hat{f}(\varepsilon, x):(\varepsilon, x) \in] 0, \eta_{j}\left[\times K_{j}\right\}} \subset \subset \Omega^{\prime}
$$

e assim podemos definir $\hat{l}_{j}$ em $\left.] 0,1\right] \times \stackrel{\circ}{K}_{j}$ por

$$
\hat{l}_{j}(\varepsilon, x)=\left\{\begin{array}{ll}
(\widehat{g}(\varepsilon, \hat{f}(\varepsilon, x)), \widehat{h}(\varepsilon, \widehat{f}(\varepsilon, x))) & , \text { se } \varepsilon \in] 0, \eta_{j}[ \\
\left(\widehat{g}\left(\varepsilon, \widehat{f}\left(\frac{\eta_{j}}{2}, x\right)\right), \widehat{h}\left(\varepsilon, \widehat{f}\left(\frac{\eta_{j}}{2}, x\right)\right)\right) & , \text { se } \varepsilon \in\left[\eta_{j}, 1\right]
\end{array} .\right.
$$

Usando 1.1.21.II temos que $\hat{l}_{j}$ é um representante de $\left(\left.(g \circ f)\right|_{K_{j}^{\circ}},\left.(h \circ f)\right|_{K_{j}^{\circ}}\right)=(g \circ$ $f, h \circ f)\left.\left.\right|_{K_{j},} \operatorname{ede}((g, h) \circ f)\right|_{K_{j}},\left.\operatorname{e~} \operatorname{assim}(g \circ f, h \circ f)\right|_{K_{j}^{\circ}} ^{\circ}=\left.((g, h) \circ f)\right|_{K_{j}^{\circ},} \cdot / /$

1.1.27 Proposição. Sejam $\Omega$ um aberto de $\mathbb{R}^{n}, \Omega^{\prime}$ um aberto de $\mathbb{R}^{p+1}$ com $V=\{z \in$ $\left.\mathbb{R}^{p}:(0, z) \in \Omega^{\prime}\right\} \neq \emptyset, f \in \mathcal{G}_{*}(\Omega ; V), g \in \mathcal{G}\left(\Omega^{\prime} ; \mathbb{R}^{s}\right)$ e $h=0$ em $\mathcal{G}(\Omega ; \mathbb{R})$, então

$$
(h, f) \in \mathcal{G}_{*}\left(\Omega ; \Omega^{\prime}\right) \quad \text { e } g \circ(h, f)=\left(\left.g\right|_{\{0\} \times V}\right) \circ f \text { em } \mathcal{G}\left(\Omega ; \mathbb{R}^{s}\right) .
$$


Demonstração. Sejam $\hat{f}$ e $\widehat{g}$ representantes, respectivamente, de $f$ e $g$, e tomemos para representante de $h$ a função $\widehat{h}$ definida em $] 0,1] \times \Omega$ por $\hat{h}(\varepsilon, x)=0$.

Seja $K \subset \subset \Omega$. Como $f \in \mathcal{G}_{*}(\Omega ; V)$ existem $K^{\prime} \subset \subset V$ e $\left.\left.\eta \in\right] 0,1\right]$ tais que

$$
\{\hat{f}(\varepsilon, x):(\varepsilon, x) \in] 0, \eta[\times K\} \subset K^{\prime}
$$

e assim

$$
\{(\widehat{h}(\varepsilon, x), \widehat{f}(\varepsilon, x)):(\varepsilon, x) \in] 0, \eta[\times K\} \subset\{0\} \times K^{\prime} \subset \subset \Omega^{\prime} .
$$

Portanto $(h, f) \in \mathcal{G}_{*}\left(\Omega ; \Omega^{\prime}\right)$.

Seja $\mathcal{K}=\left(K_{j}\right)_{j \in N}$ uma seqüência exaustiva de compactos para $\Omega$. Como $(h, f) \varepsilon$ $\mathcal{G}_{*}\left(\Omega ; \Omega^{\prime}\right)$ existe $\left(\eta_{j}\right)_{j \in N}$ tal que $1 \geq \eta_{j}>\eta_{j+1}>0$ e

$$
\overline{\{(\hat{h}(\varepsilon, x), \hat{f}(\varepsilon, x)):(\varepsilon, x) \in] 0, \eta_{j}\left[\times K_{j}\right\}} \subset \subset \Omega^{\prime},
$$

e assim podemos definir em $] 0,1] \times \stackrel{\circ}{K}_{j}$ a aplicação

$$
\widehat{l}_{j}(\varepsilon, x)=\left\{\begin{array}{ll}
\hat{g}(\varepsilon, \hat{h}(\varepsilon, x), \widehat{f}(\varepsilon, x))=\widehat{g}(\varepsilon, 0, \widehat{f}(\varepsilon, x)) & , \text { se } \varepsilon \in] 0, \eta_{j}[ \\
\hat{g}\left(\varepsilon, \hat{h}\left(\frac{\eta_{j}}{2}, x\right), \widehat{f}\left(\frac{\eta_{j}}{2}, x\right)\right)=\widehat{g}\left(\varepsilon, 0, \widehat{f}\left(\frac{\eta_{j}}{2}, x\right)\right) & , \text { se } \varepsilon \in\left[\eta_{j}, 1\right]
\end{array},\right.
$$

para todo $j \in \mathbb{N}$.

Fixemos $j \in \mathbb{N}$. Por 1.1.21.II temos $\hat{l}_{j}$ é um representante de $\left.(g \circ(h, f))\right|_{K_{j}^{\circ}}$ e de $\left.\left(\left.g\right|_{\{0\} \times V} \circ f\right)\right|_{K_{j}^{\circ}} \cdot$

Portanto $\left.(g \circ(h, f))\right|_{K_{j}^{\circ}}=\left.\left(\left.g\right|_{\{0\} \times V} \circ f\right)\right|_{K_{j}^{\circ}}$ para todo $j \in \mathbb{N}$, e assim concluímos que $g \circ(h, f)=\left.g\right|_{\{0\} \times V} \circ f(1.1 .20 . \mathrm{I}) . / /$

1.1.28 Proposição. Sejam $\Omega$ um aberto de $\mathbb{R}^{n}, \Omega^{\prime}$ um aberto de $\mathbb{R}^{p}$ e $\Omega^{\prime \prime}$ um aberto de $\mathbb{R}^{s}$. Se $f \in \mathcal{G}_{*}\left(\Omega ; \Omega^{\prime}\right), g \in \mathcal{G}_{*}\left(\Omega^{\prime} ; \Omega^{\prime \prime}\right)$ e $h \in \mathcal{G}\left(\Omega^{\prime \prime} ; \mathbb{R}^{r}\right)$, então $g \circ f \in \mathcal{G}_{*}\left(\Omega ; \Omega^{\prime \prime}\right)$ e $h \circ(g \circ f)=(h \circ g) \circ f$.

Demonstração. Seja $\mathcal{K}=\left(K_{j}\right)_{j \in N}$ uma seqüência exaustiva de compactos para $\Omega$.

Provaremos que $\left.(g \circ f)\right|_{K_{j}} \in \mathcal{G}_{*}\left(\Omega ; \Omega^{\prime \prime}\right)$ para todo $j \in \mathbb{N}$, e assim, por 1.1.20.III, teremos que $g \circ f \in \mathcal{G}_{*}\left(\Omega ; \Omega^{\prime \prime}\right)$. 
$\operatorname{Sejam}\left(\eta_{j}\right)_{j \in N}$ e $\widehat{h}_{j}, j \in \mathbb{N}$, como em 1.1.21. Então $\hat{h}_{j}$ é um representante de $\left.(g \circ f)\right|_{K_{j}^{\circ}}$, Fixemos $j \in \mathbb{N}$.

Seja $K \subset \subset \stackrel{\circ}{K_{j}}$. Como $f \in \mathcal{G}_{*}\left(\Omega ; \Omega^{\prime}\right)$, existem $K^{\prime} \subset \subset \Omega^{\prime}$ e $\left.\bar{\eta} \in\right] 0, \eta_{j}[$ tais que

$$
\{\widehat{f}(\varepsilon, x):(\varepsilon, x) \in] 0, \bar{\eta}[\times K\} \subset K^{\prime} .
$$

Como $g \in \mathcal{G}_{*}\left(\Omega^{\prime} ; \Omega^{\prime \prime}\right)$, existem $K^{\prime \prime} \subset \subset \Omega^{\prime \prime}$ e $\left.\eta \in\right] 0, \bar{\eta}[$ tais que

$$
\{\hat{g}(\varepsilon, y):(\varepsilon, y) \in] 0, \eta\left[\times K^{\prime}\right\} \subset K^{\prime \prime}
$$

Portanto

$$
\left\{\widehat{h}_{j}(\varepsilon, x):(\varepsilon, x) \in\right] 0, \eta[\times K\}=\{\widehat{g}(\varepsilon, \widehat{f}(\varepsilon, x)):(\varepsilon, x) \in] 0, \eta[\times K\} \subset K^{\prime \prime} \subset \subset \Omega^{\prime \prime}
$$

e $\left.\operatorname{assim}(g \circ f)\right|_{K_{,}^{\circ}} \in \mathcal{G}_{*}\left(\Omega ; \Omega^{\prime \prime}\right)$.

Provaremos a seguir que $h \circ(g \circ f)=(h \circ g) \circ f$.

Sejam $\hat{h}, \hat{g}$ e $\hat{f}$ representantes, respectivamente, de $h, g$ e $f$ e seja $\left(\eta_{j}\right)_{j \in N}$ como em 1.1.21.

Dado $j \in \mathbb{N}$, seja $L_{j}=\overline{\{\widehat{f}(\varepsilon, x):(\varepsilon, x) \in] 0, \eta_{j}\left[\times K_{j}\right\}} \subset \subset \Omega^{\prime}$, e como $g \in \mathcal{G}_{*}\left(\Omega^{\prime} ; \Omega^{\prime \prime}\right)$ existe $\left.\bar{\eta}_{j} \in\right] 0, \eta_{j}[$ tal que

$$
\overline{\{\hat{g}(\varepsilon, y):(\varepsilon, y) \in] 0, \bar{\eta}_{j}\left[\times L_{j}\right\}} \subset \subset \Omega^{\prime \prime},
$$

e assim podemos definir $\hat{l}_{j}$ em $\left.] 0,1\right] \times \stackrel{\circ}{K_{j}}$ por

$$
\hat{l}_{j}(\varepsilon, x)=\left\{\begin{array}{ll}
\widehat{h}(\varepsilon, \widehat{g}(\varepsilon, \hat{f}(\varepsilon, x))) & , \text { se } \varepsilon \in] 0, \bar{\eta}_{j}[ \\
\widehat{h}\left(\varepsilon, \widehat{g}\left(\frac{\bar{\eta}_{j}}{2}, \widehat{f}\left(\frac{\bar{\eta}_{j}}{2}, x\right)\right)\right) & , \text { se } \varepsilon \in\left[\bar{\eta}_{j}, 1\right]
\end{array} .\right.
$$

Usando 1.1.21.II, 1.1.22.III e 1.1.24 temos que $\hat{l}_{j}$, é um representante de $h \circ(g \circ$

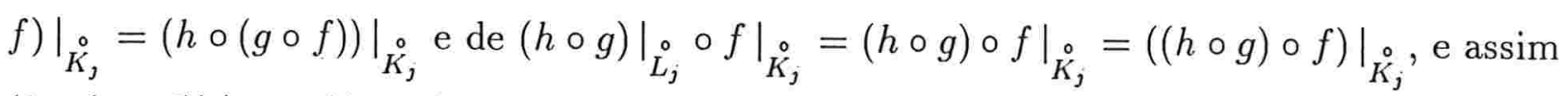
$\left.(h \circ(g \circ f))\right|_{K_{j}}=\left.((h \circ g) \circ f)\right|_{K_{j}}$, para todo $j \in \mathbb{N}$.

Portanto, por 1.1.20.I, temos que $(h \circ g) \circ f=h \circ(g \circ f) . / /$

Na seção 1.2 estabeleceremos certos fatos a respeito de aplicações generalizadas inversíveis e para tanto precisaremos de uma caracterização dos inversos multiplicativos de 
$\mathcal{G}(\Omega ; \mathbb{R})$ encontrada em [14], sendo $\Omega$ um aberto de $\mathbb{R}^{n}$. A definição abaixo é um caso particular da definição geral de inverso multiplicativo em um anel unitário e comutativo.

1.1.29 Definição. Seja $\Omega$ um aberto de $\mathbb{R}^{n}$. Dizemos que $f \in \mathcal{G}(\Omega ; \mathbb{R})$ tem inverso multiplicativo em $\mathcal{G}(\Omega ; \mathbb{R})$ se, e somente se, existe $g \in \mathcal{G}(\Omega ; \mathbb{R})$ tal que $f g=1$, isto é, a função moderada $\widehat{h}$ definida em $] 0,1] \times \Omega$ por $\widehat{h}(\varepsilon, x)=\hat{f}(\varepsilon, x) \hat{g}(\varepsilon, x)-\hat{1}(\varepsilon, x)$ pertence a $\mathcal{N}[\Omega ; \mathbb{R}]$, sendo $\hat{f}$ um representante de $f, \hat{g}$ um representante de $g$ e $\hat{1}(\varepsilon, x)=1$, para todo $(\varepsilon, x) \in] 0,1] \times \Omega$.

Em [14] uma função que satisfaz 1.1.29 é dita ser um elemento inversível. Preferimos dizer que a função tem inverso multiplicativo para não confundir com a definição dada em 1.2.1. A caracterização que usaremos é a seguinte:

1.1.30 Proposição. Sejam $\Omega$ um aberto de $\mathbb{R}^{n}, f \in \mathcal{G}(\Omega ; \mathbb{R})$ e $\hat{f}$ um representante de f. Se $\hat{f}(10,1] \times \Omega) \subset \mathbb{R}^{*}$, então as seguintes asserções sấo equivalentes:

(a) $f$ tem inverso multiplicativo em $\mathcal{G}(\Omega ; \mathbb{R})$;

(b) se $\widehat{f}_{1}$ é um representante de $f$ com $\left.\left.\widehat{f}_{1}(] 0,1\right] \times \Omega\right) \subset \mathbb{R}^{*}$, então $\frac{1}{\hat{f}_{1}}$ pertence a $\mathcal{E}_{M}[\Omega ; \mathbb{R}] ;$

(c) dado qualquer $K \subset \subset \Omega$, existem $\eta \in] 0,1]$ e uma função $\mu$ definida em $] 0,1] e$ com valores em $\mathbb{R}_{+}^{*}$ tais que $\frac{1}{\mu} \in \mathcal{E}_{M}(\mathbb{R})$ e $\mu(\varepsilon) \leq \inf \{|\widehat{f}(\varepsilon, x)|: x \in K\}$ para todo $\varepsilon \in] 0, \eta[$

(d) se $\widehat{f}_{1}$ é um representante de $f$, então dado qualquer $K \subset \subset \Omega$, existem $\left.\left.\eta \in\right] 0,1\right]$ e uma função $\mu$ definida em ]0,1] e com valores em $\mathbb{R}_{+}^{*}$ tais que $\frac{1}{\mu} \in \mathcal{E}_{M}(\mathbb{R})$ e

$$
\left.\mu(\varepsilon) \leq \inf \left\{\left|\widehat{f}_{1}(\varepsilon, x)\right|: x \in K\right\}, \text { para todo } \varepsilon \in\right] 0, \eta[
$$

Se $\hat{f}(] 0,1] \times \Omega) \subset \mathbb{R}^{*}$ e também vale a asserção (c) acima, então a classe de $\frac{1}{\widehat{f}}$ em $\mathcal{G}(\Omega ; \mathbb{R})$ é o inverso multiplicativo de $f$ em $\mathcal{G}(\Omega ; \mathbb{R})$. 
Demonstração. Ver [14]. //

Neste trabalho usaremos freqüentemente os seguintes corolários:

1.1.31 Corolário. Sejam $\Omega$ um aberto de $\mathbb{R}^{n}$ e $f \in \mathcal{G}(\Omega ; \mathbb{R})$. Se existem $\left.\left.\tau \in\right] 0,1\right]$ e um representante $\hat{f}$ de $f$ tal que $\hat{f}(] 0, \tau[\times \Omega) \subset \mathbb{R}^{*}$, então as seguintes asserções são equivalentes:

(a) $f$ tem inverso multiplicativo em $\mathcal{G}(\Omega ; \mathbb{R})$;

(b) dado qualquer $K \subset \subset \Omega$, existem $\eta \in] 0, \tau[$ e uma função $\mu$ definida em $] 0,1] e$ com valores em $\mathbb{R}_{+}^{*}$ tais que

$$
\left.\frac{1}{\mu} \in \mathcal{E}_{M}(\mathbb{R}) \text { e } \mu(\varepsilon) \leq \inf \{|\hat{f}(\varepsilon, x)|: x \in K\}, \text { para todo } \varepsilon \in\right] 0, \eta[\text {. }
$$

Demonstração. Basta observar que a função $\hat{h}$ definida em $] 0,1] \times \Omega$ por

$$
\widehat{h}(\varepsilon, x)= \begin{cases}\hat{f}(\varepsilon, x) & , \text { se } \varepsilon \in] 0, \tau[ \\ \hat{f}\left(\frac{\tau}{2}, x\right) & , \text { se } \varepsilon \in[\tau, 1]\end{cases}
$$

é um representante de $f$ e usar 1.1.30. //

1.1.32 Corolário. Sejam $\Omega$ um aberto de $\mathbb{R}^{n}, f \in \mathcal{G}(\Omega ; \mathbb{R})$ e $\widehat{f}$ um representante de $f$ tal que

(i) existe $\tau \in] 0,1]$ com $\hat{f}(] 0, \tau[\times \Omega) \subset \mathbb{R}^{*}$;

(ii) dado qualquer $K \subset \subset \Omega$, existem $a>0$ e $\eta \in] 0,1]$ satisfazendo

$$
|\widehat{f}(\varepsilon, x)| \geq a, \text { para todo }(\varepsilon, x) \in] 0, \eta[\times K \text {. }
$$

Então $f$ tem inverso multiplicativo em $\mathcal{G}(\Omega ; \mathbb{R})$.

Demonstração. Seja $K \subset \subset \Omega$ e tomemos $a>0$ e $\eta \in] 0,1]$ como em (ii).

Seja $\mu$ a função definida em $] 0,1]$ por $\mu(\varepsilon)=a$. Então

$$
\left.\frac{1}{\mu} \in \mathcal{E}_{M}(\mathbb{R}) \text { e } 0<\mu(\varepsilon)=a \leq|\widehat{f}(\varepsilon, x)|, \text { para todo }(\varepsilon, x) \in\right] 0, \eta[\times K .
$$


Portanto, por 1.1.31, concluímos que $f$ tem inverso multiplicativo em $\mathcal{G}(\Omega ; \mathbb{R}) . / /$

A seguir apresentaremos dois resultados relacionados com o conceito de inverso multiplicativo, que não estão em [14] mas que serão úteis neste trabalho.

1.1.33 Proposição. Sejam $\left.t_{o} \in \mathbb{R}, r>0, I=\right] t_{o}-r, t_{o}+r\left[, \Omega\right.$ um aberto de $\mathbb{R}^{n} e$ $f \in \mathcal{G}(I \times \Omega ; \mathbb{R})$. Denotando por $(t, x)=\left(t, x_{1}, \ldots, x_{n}\right)$ um ponto genérico de $I \times \Omega$ tem-se que, se existem um representante $\hat{f}$ de $f, \tau \in] 0,1]$ e $M>0$ satisfazendo

(i) $r M<\inf \left\{\left|\widehat{f}\left(\varepsilon, t_{o}, x\right)\right|:(\varepsilon, x) \in\right] 0, \tau[\times \Omega\}$;

(ii) $\sup \left\{\left|\frac{\partial \hat{f}}{\partial t}(\varepsilon, t, x)\right|:(\varepsilon, t, x) \in\right] 0, \tau[\times I \times \Omega \iota\} \leq M$,

então,

(I) existe $a>0$ tal que $|\widehat{f}(\varepsilon, t, x)|>a$, para todo $(\varepsilon, t, x) \in] 0, \tau[\times I \times \Omega$;

(II) $f$ tem inverso multiplicativo em $\mathcal{G}(I \times \Omega ; \mathbb{R})$.

Demonstração. A asserção (II) é uma conseqüência de (I) e de 1.1.32. Provaremos (I).

$\operatorname{Sejam} p=\inf \left\{\left|\widehat{f}\left(\varepsilon, t_{o}, x\right)\right|: x \in \Omega\right.$ e $\left.\varepsilon \in\right] 0, \tau[\}$ e $a=p-M r$.

Fixemos $(t, x) \in I \times \Omega$ e $\varepsilon \in] 0, \tau[$. Então, usando o Teorema do Valor Médio, existe $\bar{t}$ entre $t_{0}$ e $t$ tal que

$$
\widehat{f}(\varepsilon, t, x)=\widehat{f}\left(\varepsilon, t_{o}, x\right)+\frac{\partial \widehat{f}}{\partial t}(\varepsilon, \bar{t}, x)\left(t-t_{o}\right),
$$

e assim, usando (i) e (ii), temos que

$$
|\widehat{f}(\varepsilon, t, x)| \geq\left|\widehat{f}\left(\varepsilon, t_{o}, x\right)\right|-\left|\frac{\partial \hat{f}}{\partial t}(\varepsilon, \bar{t}, x)\right|\left|t-t_{o}\right| \geq p-M r=a>0,
$$

o que prova (I). //

O resultado a seguir é conhecido para anéis comutativos e unitários e o enunciaremos no caso particular do anel $\mathcal{G}(\Omega ; \mathbb{R})$, sendo $\Omega$ um aberto de $\mathbb{R}^{n}$. 
1.1.34 Proposição. Sejam $\Omega$ um aberto de $\mathbb{R}^{n}$ e $A=\left(f_{i j}\right)_{1 \leq i, j \leq n}$ uma matriz $n \times n$ com coeficientes $f_{i j}$ pertencentes a $\mathcal{G}(\Omega ; \mathbb{R})$. Se $\operatorname{det} A$ tem inverso multiplicativo em $\mathcal{G}(\Omega ; \mathbb{R})$, então o sistema

$$
\begin{array}{cccc}
X_{1} f_{11}+X_{2} f_{12}+ & \ldots & +X_{n} f_{1 n}= & 0 \\
X_{1} f_{21}+X_{2} f_{22}+ & \ldots & +X_{n} f_{2 n}= & 0 \\
\vdots & \vdots & \vdots & \vdots \\
X_{1} f_{n 1}+X_{2} f_{n 2}+ & \ldots+X_{n} f_{n n}= & 0
\end{array}
$$

admite somente a solução trivial, isto é, $X_{i}=0$, para todo $1 \leq i \leq n$.

Demonstração. Ver [13]. //

\subsection{Funções generalizadas inversíveis}

Nesta seção apresentaremos uma condição necessária para que uma aplicação generalizada seja inversível (1.2.12) e analisaremos, com vistas a aplicação posterior na resolução das equações de Hamilton-Jacobi, o seguinte problema:

Problema: Sejam $\Omega$ e $\Omega^{\prime}$ abertos de $\mathbb{R}^{n}$ e $f \in \mathcal{G}_{*}\left(\Omega ; \Omega^{\prime}\right)$ tal que existem um representante $\hat{f}$ de $f$ e $\tau \in] 0,1]$ satisfazendo:

(i) $\{\widehat{f}(\varepsilon, x): x \in \Omega\}=\Omega^{\prime}$, para todo $\left.\varepsilon \in\right] 0, \tau[$;

(ii) a aplicação $\widehat{f}(\varepsilon,$.$) é uma aplicação inversível com aplicação inversa g_{\varepsilon} \in \mathcal{C}^{\infty}\left(\Omega^{\prime} ; \Omega\right)$, para todo $\varepsilon \in] 0, \tau[$.

A aplicação generalizada $f$ é uma aplicação generalizada inversível ?

Para o caso particular em que $f$ tem um representante da forma $\widehat{f}(\varepsilon, x)=h(\varepsilon) \varphi(x)$ conseguimos uma resposta completa (1.2.4). Para o caso geral obtivemos respostas parciais (1.2.9 e 1.2.15), mas que são suficientes para o nosso propósito. O teorema apresentado em 1.2.15 nos parece de mais fácil utilização, pois as suas hipóteses não envolvem as derivadas parciais das componentes do possível representante de $f^{-1}$. 
Alguns dos resultados aqui apresentados serão utilizados no capítulo 2.

Se $\Omega$ é um aberto de $\mathbb{R}^{n}$, denotaremos por $1_{\Omega}$ a classe da aplicação moderada $\hat{1}_{\Omega}$ em $\mathcal{G}\left(\Omega ; \mathbb{R}^{n}\right)$, sendo $\hat{1}_{\Omega}$ definida em $\left.] 0,1\right] \times \Omega$ e com valores em $\mathbb{R}^{n}$ dada por $\hat{1}_{\Omega}(\varepsilon, x)=x$.

1.2.1 Definição. Sejam $\Omega$ e $\Omega^{\prime}$ abertos de $\mathbb{R}^{n}$ e $f \in \mathcal{G}_{*}\left(\Omega ; \Omega^{\prime}\right)$. Dizemos que $f$ é uma aplicação generalizada inversível (ou simplesmente uma aplicação inversível) se, e somente se, existe $g \in \mathcal{G}_{*}\left(\Omega^{\prime} ; \Omega\right)$ tal que $f \circ g=1_{\Omega^{\prime}}$ e $g \circ f=1_{\Omega}$.

Se $f$ é uma aplicação inversível, então, por 1.1.28, existe uma única $g$ como em 1.2.1. Esta aplicação $g$ é chamada aplicação inversa de $f$ e é denotada por $f^{-1}$. Quando $n=1$ dizemos que $f$ é uma função generalizada inversível (ou simplesmente uma função inversível).

1.2.2 Exemplo. Sejam $\Omega$ e $\Omega^{\prime}$ abertos de $\mathbb{R}^{n}, \varphi \in \mathcal{C}^{\infty}\left(\Omega ; \Omega^{\prime}\right)$ uma aplicação inversível com aplicação inversa $\psi \in \mathcal{C}^{\infty}\left(\Omega^{\prime} ; \Omega\right), \hat{f}$ a aplicação moderada definida em $\left.] 0,1\right] \times \Omega$ por $\hat{f}(\varepsilon, x)=\varphi(x)$ e $f$ a classe de $\hat{f}$ em $\mathcal{G}\left(\Omega ; \mathbb{R}^{n}\right)$. Então $f \in \mathcal{G}_{*}\left(\Omega ; \Omega^{\prime}\right)$, f é uma aplicação inversível e $f^{-1}$ é a classe da aplicação $\hat{g}$ em $\mathcal{G}\left(\Omega^{\prime} ; \mathbb{R}^{n}\right)$, sendo $\hat{g}$ definida em $\left.] 0,1\right] \times \Omega^{\prime}$ por $\hat{g}(\varepsilon, y)=\psi(y)$.

Para fornecer exemplos mais significativos de aplicações generalizadas inversíveis e começar a estudar o problema proposto no início dessa seção apresentamos o seguinte resultado:

1.2.3 Proposição. Sejam $\Omega$ e $\Omega^{\prime}$ abertos de $\mathbb{R}^{n}$ e $f \in \mathcal{G}_{*}\left(\Omega ; \Omega^{\prime}\right)$ tal que existem um representante $\hat{f}$ de $f$ e $\tau \in] 0,1]$ satisfazendo:

(i) $\{\widehat{f}(\varepsilon, x): x \in \Omega\}=\Omega^{\prime}$, para todo $\left.\varepsilon \in\right] 0, \tau[$;

(ii) $\widehat{f}(\varepsilon,$.$) é uma aplicação inversível e a sua aplicação inversa g_{\varepsilon}$ pertence a $\mathcal{C}^{\infty}\left(\Omega^{\prime} ; \Omega\right)$, para todo $\varepsilon \in] 0, \tau[$. 
Seja $\widehat{g}$ uma aplicação definida em $] 0,1] \times \Omega^{\prime}$ e com valores em $\Omega$ tal que $\hat{g}(\varepsilon,)=.g_{\varepsilon}$ para todo $\varepsilon \in] 0, \tau[$. Se $\hat{g}$ é tal que

(iii) $\hat{g} \in \mathcal{E}_{M}\left[\Omega^{\prime} ; \mathbb{R}^{n}\right]$;

(iv) a classe de $\hat{g}$ em $\mathcal{G}\left(\Omega^{\prime} ; \mathbb{R}^{n}\right)$ pertence a $\mathcal{G}_{*}\left(\Omega^{\prime} ; \Omega\right)$,

então $f$ é uma aplicaçâo inversivel e $f^{-1}$ é a classe de $\hat{g}$ em $\mathcal{G}\left(\Omega^{\prime} ; \mathbb{R}^{n}\right)$.

Demonstração. Basta usar a definição de aplicação inversível. //

Uma maneira simples de construir aplicações generalizadas é considerar a classe de aplicações moderadas do tipo $\hat{f}(\varepsilon, x)=h(\varepsilon) \varphi(x)$, sendo $\varphi$ uma aplicação $\mathcal{C}^{\infty}$ e $h \in \mathcal{E}_{M}(\mathbb{R})$ (1.1.16). Estudando essas funções obtivemos a seguinte proposição:

1.2.4 Proposição. Sejam $\Omega$ um aberto de $\mathbb{R}^{n}, \varphi \in \mathcal{C}^{\infty}\left(\Omega ; \mathbb{R}^{n}\right)$ uma aplicação inversível com aplicação inversa $\varphi^{-1} \in \mathcal{C}^{\infty}\left(\mathbb{R}^{n} ; \Omega\right), h \in \mathcal{E}_{M}(\mathbb{R}), \hat{f}$ a aplicação moderada definida em $] 0,1] \times \Omega$ por $\hat{f}(\varepsilon, x)=h(\varepsilon) \varphi(x)$ e f a classe de $\hat{f}$ em $\mathcal{G}\left(\Omega ; \mathbb{R}^{n}\right)$. São equivalentes as seguintes asserções:

(a) $f \in \mathcal{G}_{*}\left(\Omega ; \mathbb{R}^{n}\right)$ e $f$ é uma função inversível;

(b) existe $\tau \in] 0,1]$ tal que $0<\inf \{|h(\varepsilon)|: \varepsilon \in] 0, \tau[\} \leq \sup \{|h(\varepsilon)|: \varepsilon \in] 0, \tau[\}<\infty$.

Demonstração. Suponhamos (a) verdadeira.

Sejam $g=f^{-1} \in \mathcal{G}_{*}\left(\mathbb{R}^{n} ; \Omega\right), \hat{g}$ um representante de $g$ e $x_{o} \in \Omega$ tal que $\left\|\varphi\left(x_{o}\right)\right\|>0$.

Como $f \in \mathcal{G}_{*}\left(\Omega ; \mathbb{R}^{n}\right)$ e $g \in \mathcal{G}_{*}\left(\mathbb{R}^{n} ; \Omega\right)$, dados $\left\{x_{o}\right\}$ e $\left\{\varphi\left(x_{o}\right)\right\}$, existem $K^{\prime} \subset \subset \mathbb{R}^{n}$, $K \subset \subset \Omega$ e $\tau \in] 0,1]$ tais que

$$
\left\{\widehat{f}\left(\varepsilon, x_{o}\right): \varepsilon \in\right] 0, \tau[\} \subset K^{\prime} \text { e }\left\{\hat{g}\left(\varepsilon, \varphi\left(x_{o}\right)\right): \varepsilon \in\right] 0, \tau[\} \subset K .
$$

Da primeira inclusão de (1) temos que

$$
\left.|h(\varepsilon)|=\frac{\left\|\hat{f}\left(\varepsilon, x_{o}\right)\right\|}{\left\|\varphi\left(x_{o}\right)\right\|} \leq \frac{1}{\left\|\varphi\left(x_{o}\right)\right\|} \sup \left\{\|y\|: y \in K^{\prime}\right\}<\infty \text {, para todo } \varepsilon \in\right] 0, \tau[,
$$

e assim para concluir (b) basta provar que, se $p=\inf \{|h(\varepsilon)|: \varepsilon \in] 0, \tau[\}$, então $p>0$. 
Suponhamos, por absurdo, $p=0$. Então existe uma seqüência $\left(\varepsilon_{n}\right)_{n \in N}$ tal que $\left.\varepsilon_{n} \in\right] 0, \tau\left[\right.$, para todo $n \in \mathbb{N}$, e $\lim _{n \uparrow \infty}\left|h\left(\varepsilon_{n}\right)\right|=0$.

Seja $r=1+\left\|\varphi\left(x_{o}\right)\right\|$. Como $g \in \mathcal{G}_{*}\left(\mathbb{R}^{n} ; \Omega\right)$, existe $\left.\eta \in\right] 0, \tau[$ tal que

$$
\overline{\left\{\widehat{g}(\varepsilon, y): y \in \overline{B_{r}(0)} \text { e } \varepsilon \in\right] 0, \eta[\}} \subset \subset \Omega,
$$

e assim podemos definir a aplicação moderada $\hat{l}$ em $] 0,1] \times B_{r}(0)$ por

$$
\hat{l}(\varepsilon, y)=\left\{\begin{array}{ll}
\hat{f}(\varepsilon, \hat{g}(\varepsilon, y)) & , \text { se } \varepsilon \in] 0, \eta[ \\
\widehat{f}\left(\varepsilon, \hat{g}\left(\frac{\eta}{2}, y\right)\right) & , \text { se } \varepsilon \in[\eta, 1]
\end{array} .\right.
$$

De 1.1.22.III e 1.1.24 temos que $\hat{l}$ é um representante de $\left.f \circ g\right|_{B_{r}(0)}=\left.(f \circ g)\right|_{B_{r}(0)}=$ $\left.1_{R^{n}}\right|_{B_{r}(0)}$, e assim usando 1.1 .11 obtemos

$$
0=\lim _{\varepsilon \downarrow 0}\left(\hat{l}\left(\varepsilon, \varphi\left(x_{o}\right)-\hat{1}_{R^{n}}\left(\varepsilon, \varphi\left(x_{o}\right)\right)\right)\right.
$$

e portanto

$$
\varphi\left(x_{o}\right)=\lim _{\varepsilon \downarrow 0} \hat{f}\left(\varepsilon, \widehat{g}\left(\varepsilon, \varphi\left(x_{o}\right)\right)\right)=\lim _{\varepsilon \downarrow 0}\left[h(\varepsilon) \varphi\left(\widehat{g}\left(\varepsilon, \varphi\left(x_{o}\right)\right)\right)\right] .
$$

Da segunda inclusão de (1) concluímos que $\left\{\varphi\left(\widehat{g}\left(\varepsilon_{n}, \varphi\left(x_{o}\right)\right)\right): n \in \mathbb{N}\right\}$ é um subconjunto limitado de $\mathbb{R}^{n}$, e assim, usando (2) e que $\lim _{n \uparrow \infty}\left|h\left(\varepsilon_{n}\right)\right|=0$ temos que

$$
0 \neq\left\|\varphi\left(x_{o}\right)\right\|=\lim _{\varepsilon \downarrow 0}\left[\left\|h(\varepsilon) \varphi\left(\widehat{g}\left(\varepsilon, \varphi\left(x_{o}\right)\right)\right)\right\|\right]=\lim _{n \uparrow \infty}\left[\left|h\left(\varepsilon_{n}\right)\right|\left\|\varphi\left(\widehat{g}\left(\varepsilon_{n}, \varphi\left(x_{o}\right)\right)\right)\right\|\right]=0,
$$

o que é um absurdo.

Portanto (b) é verdadeira.

Provaremos agora que (b) implica (a).

Sejam $\tau$ como em (b), $p=\inf \{|h(\varepsilon)|: \varepsilon \in] 0, \tau[\}$ e $q=\sup \{|h(\varepsilon)|: \varepsilon \in] 0, \tau[\}$.

Usando que

$$
\|\hat{f}(\varepsilon, x)\|=\mid h(\varepsilon)\|\| \varphi(x)\|\leq q\| \varphi(x) \|, \text { para todo }(\varepsilon, x) \in] 0, \tau[\times \Omega
$$

é fácil verificar que $f \in \mathcal{G}_{*}\left(\Omega ; \mathbb{R}^{n}\right)$.

Seja $\hat{g}$ a aplicação definida em $] 0,1] \times \mathbb{R}^{n}$ por

$$
\hat{g}(\varepsilon, y)= \begin{cases}\varphi^{-1}\left(\frac{y}{h(\varepsilon)}\right) & , \text { se } \varepsilon \in] 0, \tau[ \\ \varphi^{-1}(y) & , \text { se } \varepsilon \in[\tau, 1]\end{cases}
$$


e suponhamos $\hat{g}=\left(\widehat{g}_{1}, \ldots, \widehat{g}_{n}\right)$ e $\varphi^{-1}=\left(\psi_{1}, \ldots, \psi_{n}\right)$. É claro que $\hat{g}(\varepsilon,.) \in \mathcal{C}^{\infty}\left(\mathbb{R}^{n} ; \Omega\right)$ e é a aplicação inversa de $\hat{f}(\varepsilon,$.$) , para todo \varepsilon \in] 0, \tau[$, e assim 1.2.3.i e 1.2.3.ii são verdadeiras. Portanto para concluir (a) basta, por 1.2.3, provar que $\hat{g}$ satisfaz 1.2.3.iii e 1.2.3.iv. Antes porém observemos que, se $\Gamma$ é a aplicação definida em $\mathbb{R}^{*} \times \mathbb{R}^{n}$ por $\Gamma(s, y)=\frac{1}{s} y$, então $\Gamma$ é contínua em $\mathbb{R}^{*} \times \mathbb{R}^{n}$ e dado $L \subset \subset \mathbb{R}^{n}$ temos que

$$
\left\{\frac{y}{h(\varepsilon)}: y \in L \text { e } \varepsilon \in\right] 0, \tau[\} \subset \Gamma([-q,-p] \times L) \cup \Gamma([p, q] \times L) \subset \subset \mathbb{R}^{n} .
$$

Sejam $K^{\prime} \subset \subset \mathbb{R}^{n}$ e $\alpha \in \mathbb{N}^{n}$. De (3) temos que

$$
\{\hat{g}(\varepsilon, y):(\varepsilon, y) \in] 0, \tau\left[\times K^{\prime}\right\} \subset \varphi^{-1}\left(\Gamma\left([-q,-p] \times K^{\prime}\right) \cup \Gamma\left([p, q] \times K^{\prime}\right)\right) \subset \subset \Omega
$$

e $\operatorname{assim} \hat{g}$ satisfaz 1.2.3.iv.

Para provar que $\hat{g}$ verifica 1.2.3.iii basta observar que

$$
\left|\partial^{\alpha} \widehat{g}_{i}(\varepsilon, y)\right|=\left|\partial^{\alpha} \psi_{i}\left(\frac{y}{h(\varepsilon)}\right)\left(\frac{1}{h(\varepsilon)}\right)^{|\alpha|}\right| \leq \frac{1}{p^{|\alpha|}}\left|\partial^{\alpha} \psi_{i}\left(\frac{y}{h(\varepsilon)}\right)\right|,
$$

que de (3) tem-se

$$
\left\{\partial^{\alpha} \psi_{i}\left(\frac{y}{h(\varepsilon)}\right):(\varepsilon, y) \in\right] 0, \tau\left[\times K^{\prime}\right\} \subset \partial^{\alpha} \psi_{i}\left(\Gamma\left([-q,-p] \times K^{\prime}\right) \cup \Gamma\left([p, q] \times K^{\prime}\right)\right) \subset \subset \mathbb{R}^{n}
$$

e portanto existe $c>0$ tal que

$$
\left.\left|\partial^{\alpha} \widehat{g}_{i}(\varepsilon, y)\right| \leq c \varepsilon^{-0}, \text { para todo }(\varepsilon, y) \in\right] 0, \tau\left[\times K^{\prime} \text { e } 1 \leq i \leq n . / /\right.
$$

Com o auxílio do resultado acima apresentamos a seguir algumas aplicações generalizadas inversíveis e outras não inversíveis.

1.2.5 Exemplo. Sejam $p \in \mathbb{N}^{*}, \Omega$ um aberto de $\mathbb{R}^{n}, \varphi \in \mathcal{C}^{\infty}\left(\Omega ; \mathbb{R}^{n}\right)$ uma aplicação inversível com inversa pertencente a $\mathcal{C}^{\infty}\left(\mathbb{R}^{n} ; \Omega\right)$ e $\widehat{f}_{1}, \widehat{f}_{2}, \widehat{f}_{3}$ e $\widehat{f}_{4}$ as aplicaçôes moderadas definidas em $] 0,1] \times \Omega$ por

$$
\begin{gathered}
\widehat{f}_{1}(\varepsilon, x)=\varepsilon^{p} \varphi(x) ; \widehat{f}_{2}(\varepsilon, x)=\operatorname{sen}\left(\frac{1}{\varepsilon^{p}}\right) \varphi(x) ; \widehat{f}_{3}(\varepsilon, x)=\frac{\operatorname{sen}\left(\varepsilon^{p}\right)}{\varepsilon^{p}} \varphi(x) ; \\
\hat{f}_{4}(\varepsilon, x)= \begin{cases}2 \cos \left(\varepsilon^{p}\right) \varphi(x) & \text {, se } \varepsilon \text { é racional } \\
3 \cos \left(\varepsilon^{p}\right) \varphi(x) & \text {, se } \varepsilon \text { é irracional }\end{cases}
\end{gathered}
$$


Se $f_{i}$ é a classe de $\hat{f}_{i}$ em $\mathcal{G}\left(\Omega ; \mathbb{R}^{n}\right)$, então $f_{i} \in \mathcal{G}_{*}\left(\Omega ; \mathbb{R}^{n}\right)$ para todo $1 \leq i \leq 4$, as aplicações $f_{1}$ e $f_{2}$ são não inversíveis e $f_{3}$ e $f_{4}$ são aplicações inversíveis.

Em 1.2.5 podemos, por exemplo, substituir $\varphi$, no caso $n=1$, pelas funções $\varphi(x)=$ $\operatorname{tg}(x)$ se $x \in]-\frac{\pi}{2}, \frac{\pi}{2}[$ ou $\varphi(x)=\ln (x)$ se $x>0$, e no caso $n=2$, pelas aplicações $\varphi(x, y)=(x-y, x+y)$ se $(x, y) \in \mathbb{R}^{2}$ ou $\varphi(x, y)=(y, \ln (y-x))$ se $(x, y) \in \mathbb{R}^{2}$ e $y>x$.

A seguir apresentamos outros exemplos de aplicações generalizadas inversíveis.

1.2.6 Exemplo. Sejam $h$ uma função definida em $] 0,1] \operatorname{com} h(] 0,1]) \subset\left[\frac{1}{2}, 1\right]$ e $\hat{f} a$ aplicação moderada definida em $] 0,1] \times] 0,1[\times] 0,1\left[\right.$ por $\hat{f}(\varepsilon, x, y)=\left(x^{h(\varepsilon)}, y^{h(\varepsilon)}\right)$. Se $f$ é a classe de $\hat{f}$ em $\mathcal{G}(] 0,1[\times] 0,1\left[; \mathbb{R}^{2}\right)$, então $f \in \mathcal{G}_{*}(] 0,1[\times] 0,1[;] 0,1[\times] 0,1[)$, $f$ é uma aplicação inversível e $f^{-1}$ é a classe da aplicação moderada $\widehat{g}(\varepsilon, x, y)=\left(x^{\frac{1}{h(\varepsilon)}}, y^{\frac{1}{h(e)}}\right)$ em $\mathcal{G}(] 0,1[\times] 0,1\left[; \mathbb{R}^{2}\right)$.

De fato, basta usar que $\frac{1}{2} \leq h(\varepsilon) \leq 1$ e 1.2 .3 .

Observamos que a função $h$ pode ser substituída, por exemplo, pela função cosseno.

O próximo resultado também fornece exemplos de aplicações generalizadas inversíveis.

1.2.7 Proposição. Sejam $h$ uma função definida em $] 0,1]$ e com valores em $\mathbb{R}$ com $h(] 0,1]) \subset] 0,1] e \frac{1}{h} \in \mathcal{E}_{M}(\mathbb{R})$, e $\hat{f}$ a função definida em $\left.\left.] 0,1\right] \times\right] 0,1[$ por

$$
\widehat{f}(\varepsilon, x)=h(\varepsilon)-\sqrt{(h(\varepsilon))^{2}+(h(\varepsilon)+1)^{2}-(h(\varepsilon)+x)^{2}} .
$$

Então

(I) $\hat{f} \in \mathcal{E}_{M}[] 0,1[; \mathbb{R}]$;

(II) se $f$ é a classe da função $\widehat{f}$ em $\mathcal{G}(] 0,1[; \mathbb{R})$, então $f \in \mathcal{G}_{*}(] 0,1[;]-1,0[)$ e $f$ é uma função inversível.

(Para todo $\varepsilon \in] 0,1]$ o gráfico de $\widehat{f}(\varepsilon,$.$) está contido na circunferência que passa pelos$ pontos $(1,0)$ e $(0,-1)$ e tem centro no ponto $(-h(\varepsilon), h(\varepsilon))$.) 
Demonstração. Notemos que

$$
0<(h(\varepsilon))^{2}<(h(\varepsilon))^{2}+(h(\varepsilon)+1)^{2}-(h(\varepsilon)+x)^{2}<(h(\varepsilon)+1)^{2}
$$

para todo $x \in] 0,1[$ e $\varepsilon \in] 0,1]$.

Portanto $\hat{f}(\varepsilon,.) \in \mathcal{C}^{\infty}(] 0,1[; \mathbb{R})$ e $\left.\hat{f}(\varepsilon,)(] 0,.1[) \subset\right]-1,0[$, para todo $\left.\varepsilon \in] 0,1\right]$.

Para obter (I) tomemos $K \subset \subset] 0,1[$ e $\alpha \in \mathbb{N}$.

Se $\alpha=0$, então

$$
\left.\left.\left|\partial^{\alpha} \widehat{f}(\varepsilon, x)\right|=|\widehat{f}(\varepsilon, x)| \leq 1=1 \varepsilon^{-0}, \text { para todo } \varepsilon \in\right] 0,1\right] .
$$

Se $\alpha>0$, temos que $\partial^{\alpha} \hat{f}(\varepsilon, x)$ é soma de produtos de elementos do conjunto

$$
\left\{\frac{1}{\sqrt{(h(\varepsilon))^{2}+(h(\varepsilon)+1)^{2}-(h(\varepsilon)+x)^{2}}}, h(\varepsilon)+x, h(\varepsilon), 1,-1\right\} .
$$

Como $\frac{1}{h} \in \mathcal{E}_{M}(\mathbb{R}), 0<h(\varepsilon)+x \leq 1+x$ e

$$
0<\frac{1}{\sqrt{(h(\varepsilon))^{2}+(h(\varepsilon)+1)^{2}-(h(\varepsilon)+x)^{2}}}<\frac{1}{h(\varepsilon)},
$$

é fácil verificar que existem $N \in \mathbb{N}, c>0$ e $\eta \in] 0,1]$ tais que

$$
\left.\left|\partial^{\alpha} \widehat{f}(\varepsilon, x)\right| \leq c \varepsilon^{-N}, \text { para todo }(\varepsilon, x) \in\right] 0, \eta[\times K .
$$

Portanto (I) é verdadeiro.

Seja $f$ a classe da função $\widehat{f}$ em $\mathcal{G}(10,1[; \mathbb{R})$.

Provaremos a seguir que $f \in \mathcal{G}_{*}(] 0,1[;]-1,0[)$.

Fixemos $x \in] 0,1\left[\right.$ e seja $l_{x}$ a função definida em $[0,1]$ por

$$
l_{x}(y)=y-\sqrt{y^{2}+(y+1)^{2}-(y+x)^{2}} .
$$

Então para todo $y \in[0,1]$ tem-se que

$$
l_{x}^{\prime}(y)=1+\frac{x-y-1}{\sqrt{y^{2}+(y+1)^{2}-(y+x)^{2}}}=1+\frac{x-y-1}{\sqrt{(x-y-1)^{2}-2\left(x^{2}-x\right)}}>0,
$$

e assim $l_{x}$ é uma função estritamente crescente. 
Portanto

$$
-\sqrt{1-x^{2}}=l_{x}(0) \leq \widehat{f}(\varepsilon, x)=l_{x}(h(\varepsilon)) \leq l_{x}(1)=1-\sqrt{5-(1+x)^{2}}
$$

para todo $\varepsilon \in] 0,1]$ e $x \in] 0,1[$.

Sejam $h_{1}$ e $h_{2}$ as funções definidas em ]0,1[ por

$$
h_{1}(x)=-\sqrt{1-x^{2}} \quad \text { e } \quad h_{2}(x)=1-\sqrt{5-(1+x)^{2}} .
$$

Então, $h_{1} \leq \hat{f}(\varepsilon,.) \leq h_{2}$, para todo $\left.\left.\varepsilon \in\right] 0,1\right]$ e, para todo $1 \leq i \leq 2$, temos que $h_{i}$ é uma função contínua em ]0,1[ e $\left.h_{i}(] 0,1[) \subset\right]-1,0[$.

Seja $K \subset \subset] 0,1\left[\right.$. Como $h_{1}$ e $h_{2}$ são funções contínuas em ]0,1[ temos que $h_{1}(K)$ e $h_{2}(K)$ são compactos, respectivamente em $h_{1}(] 0,1[)$ e em $h_{2}(] 0,1[)$, e portanto compactos em $]-1,0\left[\right.$. Sejam $c$ e $d$ números reais tais que $\left.h_{1}(K) \cup h_{2}(K) \subset[c, d] \subset\right]-1,0[$. Então

$$
\left.\left.c \leq h_{1}(x) \leq \hat{f}(\varepsilon, x) \leq h_{2}(x) \leq d, \text { para todo }(\varepsilon, x) \in\right] 0,1\right] \times K
$$

Portanto

$$
\{\widehat{f}(\varepsilon, x):(\varepsilon, x) \in] 0,1] \times K\} \subset[c, d] \subset \subset]-1,0[
$$

e assim temos que $f \in \mathcal{G}_{*}(] 0,1[;]-1,0[)$.

Provaremos, a seguir, que se verificam as hipóteses de 1.2.3.

Fixemos $\varepsilon \in] 0,1]$ e seja $g_{\varepsilon}$ a função definida em $]-1,0[$ por

$$
g_{\varepsilon}(y)=-h(\varepsilon)+\sqrt{(h(\varepsilon))^{2}+(h(\varepsilon)+1)^{2}-(h(\varepsilon)-y)^{2}} .
$$

Então $g_{\varepsilon}$ é a função inversa de $\widehat{f}(\varepsilon,$.$\left.\left.) para todo \varepsilon \in\right] 0,1\right]$, e como $g_{\varepsilon}(y)=-\widehat{f}(\varepsilon,-y)$ para todo $(\varepsilon, y) \in] 0,1] \times$ ] $-1,0$ [ é fácil verificar que a função $\widehat{g}$ definida em ]0,1]× $-1,0[$ por $\hat{g}(\varepsilon,)=.g_{\varepsilon}$ pertence a $\mathcal{E}_{M}[]-1,0[; \mathbb{R}]$ e a classe de $\hat{g}$ em $\mathcal{G}(]-1,0[; \mathbb{R})$ pertence a $\mathcal{G}_{*}(]-1,0[;] 0,1[)$.

Portanto por 1.2.3 concluímos que $f$ é uma função inversível. //

Completamos o resultado acima exibindo funções $h$ verificando as hipóteses do mesmo. 
1.2.8 Exemplo. Seja $p \in \mathbb{N}^{*}$. Então em 1.2 .7 a função $h$ pode ser substituída, por exemplo, por uma das seguintes funções:

$$
h_{1}(\varepsilon)=\varepsilon^{p}, h_{2}(\varepsilon)=\cos \left(\varepsilon^{p}\right) \text { e } h_{3}(\varepsilon)=\operatorname{sen}\left(\varepsilon^{p}\right) .
$$

De fato, basta verificar que $\frac{1}{h_{i}} \in \mathcal{E}_{M}(\mathbb{R})$ para todo $1 \leq i \leq 3$, o que é imediato para $h_{1}$ e $h_{2}$ pois

$$
\left.\left.\left|\frac{1}{h_{1}(\varepsilon)}\right|=\frac{1}{\varepsilon^{p}} \text { e }\left|\frac{1}{h_{2}(\varepsilon)}\right| \leq \frac{1}{\cos (1)} \text {, para todo } \varepsilon \in\right] 0,1\right]
$$

Para $h_{3}$ é necessário observar que $\lim _{\varepsilon \downarrow 0}\left|\frac{\varepsilon^{p+1}}{h_{3}(\varepsilon)}\right|=0$, e assim existe $\left.\left.\eta \in\right] 0,1\right]$ tal que $\left|\frac{1}{h_{3}(\varepsilon)}\right| \leq$ $\frac{1}{\varepsilon^{p+1}}$, para todo $\left.\varepsilon \in\right] 0, \eta[$.

Sejam $\Omega$ e $\Omega^{\prime}$ abertos de $\mathbb{R}^{n}, f \in \mathcal{G}_{*}\left(\Omega ; \Omega^{\prime}\right), \widehat{f}$ satisfazendo 1.2 .3 .i e 1.2 .3 .ii e $\hat{g}$ a aplicação definida em 1.2.3. Se $\hat{g} \in \mathcal{E}_{M}\left[\Omega^{\prime} ; \mathbb{R}^{n}\right]$ podemos chamar de $g$ a classe da aplicação $\hat{g}$ em $\mathcal{G}\left(\Omega^{\prime} ; \mathbb{R}^{n}\right)$ e sabemos que, se $g \in \mathcal{G}_{*}\left(\Omega^{\prime} ; \Omega\right)$, então $f$ é uma aplicação inversível e $g=f^{\sim 1}(1.2 .3)$. Surge então a seguinte pergunta: Se $g \notin \mathcal{G}_{*}\left(\Omega^{\prime} ; \Omega\right)$ será que $f$ pode ser uma aplicação inversível ? A resposta é o seguinte resultado:

1.2.9 Teorema. Sejam $\Omega$ e $\Omega^{\prime}$ abertos de $\mathbb{R}^{n}$ e $f \in \mathcal{G}_{*}\left(\Omega ; \Omega^{\prime}\right)$ tal que existem um representante $\widehat{f}$ de $f$ e $\tau \in] 0,1]$ satisfazendo:

(i) $\{\widehat{f}(\varepsilon, x): x \in \Omega\}=\Omega^{\prime}$, para todo $\left.\varepsilon \in\right] 0, \tau[$;

(ii) $\widehat{f}\left(\varepsilon\right.$, .) é uma aplicação inversível e a sua aplicação inversa $g_{\varepsilon}$ pertence a $\mathcal{C}^{\infty}\left(\Omega^{\prime} ; \Omega\right)$, para todo $\varepsilon \in] 0, \tau[$;

(iii) $\widehat{g}$ definida em $] 0,1] \times \Omega^{\prime}$ e com valores em $\mathbb{R}^{n}$ dada por $\hat{g}(\varepsilon,)=.g_{\varepsilon}$ para todo $\varepsilon \in] 0, \tau\left[\right.$ e $\widehat{g}(\varepsilon,)=.g_{\frac{\tau}{2}}$ para todo $\varepsilon \in[\tau, 1]$, pertence a $\mathcal{E}_{M}\left[\Omega^{\prime} ; \mathbb{R}^{n}\right]$.

Se $g$ é a classe de $\widehat{g}$ em $\mathcal{G}\left(\Omega^{\prime} ; \mathbb{R}^{n}\right)$, então são equivalentes as seguintes afirmaçôes:

(a) $f$ é uma aplicação inversível; 
(b) $g \in \mathcal{G}_{*}\left(\Omega^{\prime} ; \Omega\right)$;

(c) $g \in \mathcal{G}_{*}\left(\Omega^{\prime} ; \Omega\right), \quad f \circ g=1_{\Omega^{\prime}} \quad e \quad g \circ f=1_{\Omega}$.

Demonstração. Provaremos, em primeiro lugar, que (a) implica (b).

Como $f$ é uma aplicação inversível, existe $h \in \mathcal{G}_{*}\left(\Omega^{\prime} ; \Omega\right)$ tal que $h \circ f=1_{\Omega}$ e $f \circ h=1_{\Omega^{\prime}}$.

Usando que $f \in \mathcal{G}_{*}\left(\Omega ; \Omega^{\prime}\right)$ e valem (i), (ii) e (iii), temos que $g \circ f=1_{\Omega}$, e assim, com o auxílio de 1.1.28, concluímos que

$$
g=g \circ 1_{\Omega^{\prime}}=g \circ(f \circ h)=(g \circ f) \circ h=1_{\Omega} \circ h=h .
$$

Como $g=h \in \mathcal{G}_{*}\left(\Omega^{\prime} ; \Omega\right)$ temos que a asserção (b) é verdadeira.

De 1.2.3 concluímos que (b) implica (c), e usando a definição de aplicação inversível temos que (c) implica (a) . //

Usando o Teorema acima obtemos o seguinte exemplo:

1.2.10 Exemplo. Seja $\hat{f}$ a função definida em $] 0,1] \times]-\frac{\pi}{2}, \frac{\pi}{2}[$ por $\hat{f}(\varepsilon, x)=\exp (\varepsilon \operatorname{tg} x)$. Então $\hat{f} \in \mathcal{E}_{M}[]-\frac{\pi}{2}, \frac{\pi}{2}[; \mathbb{R}]$ e se $f$ é a classe de $\widehat{f}$ em $\mathcal{G}(]-\frac{\pi}{2}, \frac{\pi}{2}[; \mathbb{R})$ tem-se que $f \in \mathcal{G}_{*}(]-\frac{\pi}{2}, \frac{\pi}{2}\left[; \mathbb{R}_{+}^{*}\right)$ e f não é uma função inversivel.

De fato, para verificar que $\hat{f} \in \mathcal{E}_{M}[]-\frac{\pi}{2}, \frac{\pi}{2}[; \mathbb{R}]$ basta usar que, se $\left.K \subset \subset\right]-\frac{\pi}{2}, \frac{\pi}{2}[$ e $a>0$ é tal que $\{\operatorname{tg} x: x \in K\} \subset[-a, a]$, então

$$
\{\exp (\varepsilon \operatorname{tg} x):(\varepsilon, x) \in] 0,1] \times K\} \subset[\exp (-a), \exp a] \subset \subset \mathbb{R}_{+}^{*},
$$

e usar que, se $n \in \mathbb{N}$, então $\widehat{f}^{(n)}(\varepsilon, x)$ é soma de produtos de elementos do conjunto

$$
\{\exp (\varepsilon \operatorname{tg} x)\} \cup\left\{\operatorname{tg}^{(s)} x: s \in \mathbb{N}\right\} \cup\left\{\varepsilon^{j}: j \in \mathbb{N}\right\} .
$$

De (1) é claro que $f \in \mathcal{G}_{*}(]-\frac{\pi}{2}, \frac{\pi}{2}\left[; \mathbb{R}_{+}^{*}\right)$. Para provar que $\widehat{f}$ satisfaz 1.2.9.i e 1.2.9.ii basta observar que $\hat{f}(\varepsilon,$.$) é uma função estritamente crescente, \lim _{x \uparrow \frac{\pi}{2}} \widehat{f}(\varepsilon, x)=\infty$ e $\lim _{x \downarrow-\frac{\pi}{2}} \hat{f}(\varepsilon, x)=$ 0 , para todo $\varepsilon \in] 0,1]$. Seja $\widehat{g}$ a função definida em $] 0,1] \times \mathbb{R}_{+}^{*}$ por $\widehat{g}(\varepsilon, y)=\operatorname{arctg}\left(\frac{\ln y}{\varepsilon}\right)$. É claro que $\widehat{g}(\varepsilon,$.$) é a função inversa de \widehat{f}(\varepsilon,$.$) e \widehat{g}(\varepsilon,.) \in \mathcal{C}^{\infty}\left(\mathbb{R}_{+}^{*} ;\right]-\frac{\pi}{2}, \frac{\pi}{2}[)$, para todo 
$\varepsilon \in] 0,1]$. Para verificar que $\hat{g} \in \mathcal{E}_{M}\left[\mathbb{R}_{+}^{*} ; \mathbb{R}\right]$ basta usar que, se $\left.\left.(\varepsilon, y) \in\right] 0,1\right] \times \mathbb{R}_{+}^{*}$ e $n=0$, então $|\widehat{g}(\varepsilon, y)|=\left|\widehat{g}^{(0)}(\varepsilon, y)\right| \leq \frac{\pi}{2}$ e, se $n \in \mathbb{N}^{*}$ e $\varphi(x)=\frac{1}{1+x^{2}}$ para todo $x \in \mathbb{R}$, então $\varphi$ é uma função limitada e $\widehat{g}^{(n)}(\varepsilon, y)$ é soma de produtos de elementos do conjunto

$$
\left\{\varphi\left(\frac{\ln y}{\varepsilon}\right)\right\} \cup\left\{\ln ^{(s)} y: s \in \mathbb{N}\right\} \cup\left\{\frac{1}{\varepsilon^{j}}: j \in \mathbb{N}\right\} \cup\{-1\} \text {. }
$$

Para concluir que $f$ é uma função não inversível basta observar que $\hat{g}$ não satisfaz 1.2.9.b para $K^{\prime}=\{2\}$.

Sejam $\Omega$ e $\Omega^{\prime}$ abertos de $\mathbb{R}^{n}, f \in \mathcal{G}_{*}\left(\Omega ; \Omega^{\prime}\right), \hat{f}$ satisfazendo 1.2.3.i e 1.2.3.ii e $\hat{g}$ a aplicação definida em 1.2.3. Se $\hat{g} \notin \mathcal{E}_{M}\left[\Omega^{\prime} ; \mathbb{R}^{n}\right]$ será que $f$ é uma aplicação inversível ? Para responder essa pergunta procuramos descobrir condições necessárias para que uma aplicação generalizada fosse uma aplicação inversível. Sabemos que uma condição necessária, mas não suficiente, para que uma aplicação $\varphi$ pertencente a $\mathcal{C}^{\infty}\left(\Omega ; \Omega^{\prime}\right)$ seja uma aplicação inversível é que a aplicação $J \varphi$ definida por $J \varphi(x)=\operatorname{det}\left(d \varphi_{x}\right)$ nunca se anule, e por conseguinte temos $\frac{1}{J \varphi} \in \mathcal{C}^{\infty}(\Omega ; \mathbb{R})$. Para o caso de uma aplicação generalizada teremos uma condição análoga. Afim de obter essa condição precisaremos definir o jacobiano de uma aplicação generalizada, e para isso é necessário que observemos o seguinte:

Sejam $\Omega$ um aberto de $\mathbb{R}^{n}, f \in \mathcal{G}\left(\Omega ; \mathbb{R}^{n}\right), \hat{f}=\left(\hat{f}_{1}, \ldots, \hat{f}_{n}\right)$ um representante de $f$ e $J \widehat{f}$ a função definida em $] 0,1] \times \Omega$ e com valores em $\mathbb{R}$, dada por

$$
J \widehat{f}(\varepsilon, x)=\operatorname{det}\left(d(\hat{f}(\varepsilon, .))_{x}\right)=\operatorname{det}\left(\frac{\partial \hat{f}_{i}}{\partial x_{j}}(\varepsilon, x)\right)_{1 \leq i, j \leq n} .
$$

É claro que $J \widehat{f} \in \mathcal{E}_{M}[\Omega ; \mathbb{R}]$. Se $\widehat{g}=\left(\widehat{g}_{1}, \ldots, \widehat{g}_{n}\right)$ é outro representante de $f$, então

$$
(J \hat{f}-J \hat{g})(\varepsilon, x)=\sum_{\sigma \in S_{n}} \varepsilon_{\sigma} d_{\sigma}(\varepsilon, x)
$$

onde $d_{\sigma}(\varepsilon, x)=\prod_{i=1}^{n} \frac{\partial \widehat{f}_{i}}{\partial x_{\sigma(i)}}(\varepsilon, x)-\prod_{i=1}^{n} \frac{\partial \widehat{g}_{i}}{\partial x_{\sigma(i)}}(\varepsilon, x), \quad S_{n}$ é o conjunto das permutações de $\{1, \ldots, n\}$ e $\varepsilon_{\sigma}$ é o sinal da permutação $\sigma$.

Do seguinte fato geral: 
Sejam $A$ um anel unitário e comutativo, I um ideal de $A$ e $\left(a_{\lambda}\right)_{\lambda \in \Lambda} e\left(b_{\lambda}\right)_{\lambda \in \Lambda}$ duas familias de elementos de $A$ indexadas por um conjunto finito não vazio $\Lambda$. Se $a_{\lambda}-b_{\lambda} \in I$ para cada $\lambda \in \Lambda$, então $\prod_{\lambda \in \Lambda} a_{\lambda}-\prod_{\lambda \in \Lambda} b_{\lambda} \in I$,

cuja prova é trivial considerando o quociente $A / I$, tem-se que $d_{\sigma} \in \mathcal{N}[\Omega ; \mathbb{R}]$, para todo $\sigma \in S_{n}$, e $\operatorname{assim} J \hat{f}-J \hat{g} \in \mathcal{N}[\Omega ; \mathbb{R}]$. Com essas considerações tem sentido a seguinte definição:

1.2.11 Definição. Sejam $\Omega$ um aberto de $\mathbb{R}^{n}$ e $f \in \mathcal{G}\left(\Omega ; \mathbb{R}^{n}\right)$. Chama-se jacobiano de $f$, e será denotado por $J f$, a classe de $J \widehat{f}$ em $\mathcal{G}(\Omega ; \mathbb{R})$, onde $J \widehat{f}$ é como acima e $\widehat{f}$ é um representante qualquer de $f$.

Usando o conceito de inverso multiplicativo (1.1.29) apresentamos, em 1.2.12, uma condição necessária, mas não suficiente, para que uma aplicação generalizada seja inversível.

1.2.12 Proposição. Sejam $\Omega$ e $\Omega^{\prime}$ abertos de $\mathbb{R}^{n}$ e $f \in \mathcal{G}_{*}\left(\Omega ; \Omega^{\prime}\right)$. Se $f$ é uma aplicação inversível, então Jf tem inverso multiplicativo em $\mathcal{G}(\Omega ; \mathbb{R})$.

Demonstração. Seja $g \in \mathcal{G}_{*}\left(\Omega^{\prime} ; \Omega\right)$ tal que $g \circ f=1_{\Omega}$ e $f \circ g=1_{\Omega^{\prime}}$.

Seja $\mathcal{K}=\left(K_{j}\right)_{j \in N}$ uma seqüência exaustiva de compactos para $\Omega$.

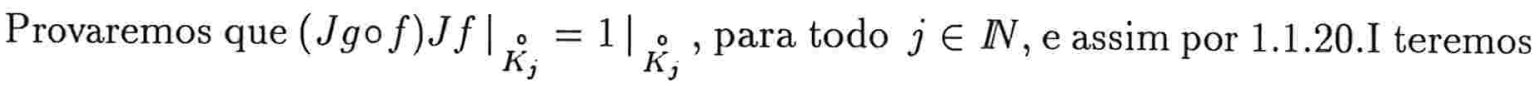
que $(J g \circ f) J f=1$, o que provará que $J f$ tem inverso multiplicativo em $\mathcal{G}(\Omega ; \mathbb{R})$.

Sejam $\hat{g}$ um representante de $g$ e $\hat{f}$ um representante de $f$.

Como $\widehat{f}$ satisfaz 1.1.18.i existe $\left(\eta_{j}\right)_{j \in N}$ uma seqüência em $\left.] 0,1\right]$ com $\eta_{j}>\eta_{j+1}$, para todo $j \in \mathbb{N}$ e

$$
\overline{\{\widehat{f}(\varepsilon, x):(\varepsilon, x) \in] 0, \eta_{j}\left[\times K_{j}\right\}} \subset \subset \Omega^{\prime} \text {, para todo } j \in \mathbb{N} \text {. }
$$

Sejam $j \in \mathbb{I}, \hat{h}_{j}$ e $\hat{l}_{j}$ as funções definidas em $\left.] 0,1\right] \times \stackrel{\circ}{K}_{j}$ por

$$
\widehat{h}_{j}(\varepsilon, x)=\left\{\begin{array}{ll}
J \hat{g}(\varepsilon, \hat{f}(\varepsilon, x)) & , \text { se } \varepsilon \in] 0, \eta_{j}[ \\
J \widehat{g}\left(\varepsilon, \hat{f}\left(\frac{\eta_{j}}{2}, x\right)\right) & , \text { se } \varepsilon \in\left[\eta_{j}, 1\right]
\end{array},\right.
$$




$$
\hat{l}_{j}(\varepsilon, x)=\left\{\begin{array}{ll}
\hat{g}(\varepsilon, \hat{f}(\varepsilon, x)) & , \text { se } \varepsilon \in] 0, \eta_{j}[ \\
\hat{g}\left(\varepsilon, \hat{f}\left(\frac{\eta_{j}}{2}, x\right)\right) & , \text { se } \varepsilon \in\left[\eta_{j}, 1\right]
\end{array} .\right.
$$

Fixemos $j \in \mathbb{N}$.

Usando 1.1.21.II temos que $\widehat{h}_{j}$ é um representante de $\left.(J g \circ f)\right|_{K_{0}}$ e $\hat{l}_{j}$ é um representante $\left.\operatorname{de}(g \circ f)\right|_{\check{K}_{j}} ^{\stackrel{\circ}{C}}$

Como $g \circ f=1_{\Omega}$ temos que $\left.(J(g \circ f))\right|_{K_{j}^{\circ}} ^{\circ}=\left.J\left(1_{\Omega}\right)\right|_{K_{j}^{\circ}} ^{\circ}=\left.1\right|_{K_{j}^{\circ}} ^{\circ}$, e $\operatorname{assim} J \hat{l}_{j}-\left.\hat{1}\right|_{] \mathbf{0 , 1} \times K_{j}} \in$ $\mathcal{N}\left[\stackrel{\circ}{K_{j}} ; \mathbb{R}\right]$.

Portanto, em $] 0, \eta_{j}\left[\times \stackrel{\circ}{K}_{j}\right.$, temos que $\widehat{h}_{j} J \hat{f}-\hat{1}=J \hat{l}_{j}-\hat{1}$, e portanto concluímos que $\left.(J g \circ f) J f\right|_{K_{j}^{\circ}}=\left.1\right|_{K_{j}^{\circ}} \cdot / /$

\subsubsection{Observação. A recíproca de 1.2.12 é falsa.}

De fato, basta verificar que, se $f$ é a função definida em 1.2.10, então $J f$ tem inverso multiplicativo em $\mathcal{G}(]-\frac{\pi}{2} ; \frac{\pi}{2}[; \mathbb{R})$. Para verificar que $J f$ tem inverso multiplicativo tomemos $K \subset \subset]-\frac{\pi}{2} ; \frac{\pi}{2}[, a$ e $b$ números reais tais que $K \subset[a, b] \subset]-\frac{\pi}{2} ; \frac{\pi}{2}[$ e $\mu$ a função definida em $] 0,1]$ por

$$
\mu(\varepsilon)=\frac{\varepsilon}{2} \min \left\{\sec ^{2} y: y \in K\right\}>0 .
$$

Então $\frac{1}{\mu} \in \mathcal{E}_{M}(\mathbb{R})$ e se $\left.\left.\eta \in\right] 0,1\right]$ é tal que $\exp (\varepsilon \operatorname{tg} a)>\frac{1}{2}$ para todo $\left.\varepsilon \in\right] 0, \eta[$, temos que $\mu(\varepsilon) \leq \varepsilon \exp (\varepsilon \operatorname{tg} a) \min \left\{\sec ^{2} y: y \in K\right\} \leq \varepsilon \exp (\varepsilon \operatorname{tg} a) \sec ^{2} x \leq \varepsilon \exp (\varepsilon \operatorname{tg} x) \sec ^{2} x=J \hat{f}(\varepsilon, x)$, para todo $(\varepsilon, x) \in] 0, \eta[\times K$, e como $J \widehat{f}(] 0,1] \times]-\frac{\pi}{2} ; \frac{\pi}{2}[) \subset \mathbb{R}_{+}^{*}$ concluímos, por 1.1.31, que $J f$ tem inverso multiplicativo em $\mathcal{G}(]-\frac{\pi}{2} ; \frac{\pi}{2}[; \mathbb{R})$.

No próximo exemplo usaremos 1.2.12 para garantir que uma certa função generalizada não é inversível.

1.2.14 Exemplo. Seja $\hat{f}$ a função definida em $] 0,1] \times \mathbb{R}$ por

$$
\hat{f}(\varepsilon, x)=\int_{0}^{x} \exp \left(\frac{-s^{2}}{\varepsilon}\right) d s .
$$


Então $\hat{f} \in \mathcal{E}_{M}[\mathbb{R} ; \mathbb{R}]$ e se, $a=\int_{0}^{\infty} \exp \left(-t^{2}\right) d t=\frac{\sqrt{\pi}}{2}$ ef é a classe de $\hat{f}$ em $\mathcal{G}(\mathbb{R} ; \mathbb{R})$ tem-se que $\hat{f}(] 0,1] \times \mathbb{R})=]-a, a\left[, f \in \mathcal{G}_{*}(\mathbb{R} ;]-a, a[)\right.$ e $f$ não é uma função inversível. De fato, para verificar que $\hat{f} \in \mathcal{E}_{M}[\mathbb{R} ; \mathbb{R}]$ basta observar que

$$
|\widehat{f}(\varepsilon, x)|=\left|\int_{0}^{x} \exp \left(\frac{-s^{2}}{\varepsilon}\right) d s\right|=\left|\sqrt{\varepsilon} \int_{0}^{x / \sqrt{\varepsilon}} \exp \left(-t^{2}\right) d t\right| \leq a \sqrt{\varepsilon} \leq \frac{a}{2},
$$

para todo $\left.(\varepsilon, x) \in] 0, \frac{1}{4}\right] \times \mathbb{R}$, que se $n \in \mathbb{N}^{*}$, então $\widehat{f}^{(n)}(\varepsilon, x)$ é soma de produtos de elementos do conjunto

$$
\left\{\exp \left(\frac{-x^{2}}{\varepsilon}\right)\right\} \cup\{x,-1\} \cup\left\{\frac{1}{\varepsilon^{j}}: j \in \mathbb{N}\right\}
$$

e que $0<\exp \left(\frac{-x^{2}}{\varepsilon}\right) \leq 1$, para todo $\left.\left.(\varepsilon, x) \in\right] 0,1\right] \times \mathbb{R}$. De (1) é claro que $f \in$ $\mathcal{G}_{*}(\mathbb{R} ;]-a, a[)$. Como $\hat{f}(\varepsilon,$.$) é uma função estritamente crescente, \lim _{x \uparrow \infty} \hat{f}(\varepsilon, x)=a \sqrt{\varepsilon}$ e $\lim _{x \downarrow-\infty} \hat{f}(\varepsilon, x)=-a \sqrt{\varepsilon}$, temos que $\left.\hat{f}(\varepsilon,).(\mathbb{R})=\right]-a \sqrt{\varepsilon}, a \sqrt{\varepsilon}[$ para todo $\left.\varepsilon \in] 0,1\right]$, e portanto $\left.\hat{f}([0,1] \times \mathbb{R})=\cup_{\varepsilon \in] 0,1]}\right]-a \sqrt{\varepsilon}, a \sqrt{\varepsilon}[=]-a, a\left[\right.$. De $J \widehat{f}(\varepsilon, x)=\exp \left(\frac{-x^{2}}{\varepsilon}\right) \neq 0$ para todo $(\varepsilon, x) \in] 0,1] \times \mathbb{R}$ e

$$
\lim _{\varepsilon \downarrow 0}\left|\frac{\varepsilon^{N}}{J \hat{f}(\varepsilon, 1)}\right|=\lim _{\varepsilon \downarrow 0}\left|\varepsilon^{N} \exp \left(\frac{1}{\varepsilon}\right)\right|=\infty, \text { para todo } N \in \mathbb{N},
$$

tem-se que $\frac{1}{J \hat{f}}$ não satisfaz 1.1.3.ii para $\alpha=n=0$ e $K=\{1\}$, e assim $\frac{1}{J \widehat{f}} \notin \mathcal{E}_{M}[\mathbb{R} ; \mathbb{R}]$. Esse fato juntamente com 1.1.30 garante que $J f$ não tem inverso multiplicativo, e portanto $f$ não é uma função inversível (1.2.12).

O resultado abaixo, além de responder parcialmente a pergunta que foi feita após 1.2.10, será uma ferramenta importante no capítulo 2 .

1.2.15 Teorema. Sejam $\Omega$ e $\Omega^{\prime}$ abertos de $\mathbb{R}^{n}$ e $f \in \mathcal{G}_{*}\left(\Omega ; \Omega^{\prime}\right)$ tal que existem um representante $\hat{f}$ de $f$ e $\tau \in] 0,1]$ satisfazendo:

(i) $\{\widehat{f}(\varepsilon, x): x \in \Omega\}=\Omega^{\prime}$, para todo $\left.\varepsilon \in\right] 0, \tau[$;

(ii) $\hat{f}(\varepsilon,$.$) é uma aplicação inversível com aplicação inversa g_{\varepsilon}$, para todo $\left.\varepsilon \in\right] 0, \tau[$; 
(iii) dado qualquer $K^{\prime} \subset \subset \Omega^{\prime}$, existem $K \subset \subset \Omega$ e $\left.\eta \in\right] 0, \tau[$ tais que

$$
\left\{g_{\varepsilon}(y):(\varepsilon, y) \in\right] 0, \eta\left[\times K^{\prime}\right\} \subset K \text {; }
$$

(iv) $J \widehat{f}(] 0, \tau[\times \Omega) \subset \mathbb{R}^{*}$.

Seja $\hat{g}$ a aplicação definida em $] 0,1] \times \Omega^{\prime}$ e com valores em $\Omega$ tal que $\hat{g}(\varepsilon,)=.g_{\varepsilon}$ para todo $\varepsilon \in] 0, \tau\left[\right.$ e $\hat{g}(\varepsilon,)=.g_{\frac{\tau}{2}}$ para todo $\varepsilon \in[\tau, 1]$. São equivalentes as seguintes afirmações:

(a) $J f$ tem inverso multiplicativo em $\mathcal{G}(\Omega ; \mathbb{R})$;

(b) $\hat{g}$ pertence a $\mathcal{E}_{M}\left[\Omega^{\prime} ; \mathbb{R}^{n}\right]$;

(c) $f$ é uma aplicação inversível e a sua aplicação inversa é a classe de $\hat{g}$ em $\mathcal{G}\left(\Omega^{\prime} ; \mathbb{I} R^{n}\right)$.

(Convém observar que a condição 1.2.15.iv é equivalente a afirmar que $\widehat{g}_{\varepsilon} \in \mathcal{C}^{\infty}\left(\Omega^{\prime} ; \Omega\right)$, para todo $\varepsilon \in] 0, \tau[$.)

Demonstração. Drovaremos, em primeiro lugar, que (a) implica (b).

Sejam $\hat{f}=\widehat{f}\left(\varepsilon, x_{1}, x_{2}, \ldots, x_{n}\right)$ e $\hat{g}=\hat{g}\left(\varepsilon, y_{1}, y_{2}, \ldots, y_{n}\right)$.

Suponhamos $\hat{f}=\left(\widehat{f}_{1}, \widehat{f}_{2}, \ldots, \widehat{f}_{n}\right)$ e $\hat{g}=\left(\widehat{g}_{1}, \hat{g}_{2}, \ldots, \widehat{g}_{n}\right)$.

Para $\varepsilon \in] 0, \eta\left[\right.$ temos, por (iv) e pelo Teorema da Função Inversa, que $g_{\varepsilon} \in \mathcal{C}^{\infty}\left(\Omega^{\prime} ; \Omega\right)$. Portanto $\hat{g}(\varepsilon,$.$) pertence a \mathcal{C}^{\infty}\left(\Omega^{\prime} ; \Omega\right)$, para todo $\left.\left.\varepsilon \in\right] 0,1\right]$.

Sejam $K^{\prime} \subset \subset \Omega^{\prime}$ e $\alpha \in \mathbb{N}^{n}$. Provaremos que existem $N \in \mathbb{N}, c>0$ e $\left.\left.\eta_{1} \in\right] 0,1\right]$ tais que

$$
\left.\sup \left\{\left|\partial^{\alpha} \widehat{g}_{l}(\varepsilon, y)\right|: y \in K^{\prime} \text { e } 1 \leq l \leq n\right\} \leq c \varepsilon^{-N} \text {, para todo } \varepsilon \in\right] 0, \eta_{1}[,
$$

e assim teremos (b).

Para demonstrar (1) tomemos $K \subset \subset \Omega$ e $\eta \in] 0, \tau[$ como em (iii), isto é

$$
\{\widehat{g}(\varepsilon, y):(\varepsilon, y) \in] 0, \eta\left[\times K^{\prime}\right\} \subset K .
$$

Portanto para $|\alpha|=0$ basta escolher $N=0, \eta_{1}=\eta$ e $c=\sup \{\|x\|: x \in K\}$.

Suponhamos $|\alpha| \geq 1$. Provaremos (1), neste caso, usando o Princípio de Indução Finita sobre $|\alpha|$. 
Para $\varepsilon \in] 0, \eta\left[\right.$ sabemos que $\hat{f}(\varepsilon, \hat{g}(\varepsilon,))=.\hat{1}_{\Omega^{\prime}}$, e assim $d \widehat{g}(\varepsilon,$.$) é a matriz inversa de$ $d \widehat{f}(\varepsilon, .)_{\hat{g}(\varepsilon, .)}$. Portanto para todo $1 \leq j, k \leq n$ temos que

$$
\frac{\partial \hat{g}_{k}}{\partial y_{j}}(\varepsilon, y)=\frac{1}{J \hat{f}(\varepsilon, \hat{g}(\varepsilon, y))} a_{j k}
$$

onde $a_{j k}$ é soma de produtos de elementos do conjunto

$$
\left\{\frac{\partial \widehat{f}_{i}}{\partial x_{s}}(\varepsilon, \hat{g}(\varepsilon, y)): 1 \leq i, s \leq n\right\} \cup\{1,-1\}
$$

Usando que $J f$ tem inverso multiplicativo em $\mathcal{G}(\Omega ; \mathbb{R})$, existem, por (iv) e por 1.1.31, $\left.\eta_{2} \in\right] 0, \eta[$ e uma função $\mu$ definida em $\left.] 0,1\right]$ e com valores em $\mathbb{R}_{+}^{*}$ tais que $\frac{1}{\mu} \in \mathcal{E}_{M}(\mathbb{R})$ e

$$
0<\mu(\varepsilon) \leq \inf \{|J \hat{f}(\varepsilon, x)|: x \in K\} \text {, para todo } \varepsilon \in] 0, \eta_{2}[\text {. }
$$

Como $\frac{1}{\mu} \in \mathcal{E}_{M}(\mathbb{R})$ e $\hat{f}_{i} \in \mathcal{E}_{M}[\Omega ; \mathbb{R}]$, para todo $1 \leq i \leq n$, existem $\bar{N} \in \mathbb{N}, \bar{c}>0$ e $\left.\eta_{3} \in\right] 0, \eta_{2}[$ tais que se $\varepsilon \in] 0, \eta_{3}[$, então

$$
0<\frac{1}{\mu(\varepsilon)} \leq \bar{c} \varepsilon^{-\bar{N}}
$$

$\sup \left\{\left|\partial^{\gamma} \widehat{f}_{i}(\varepsilon, x)\right|: x \in K, 1 \leq i \leq n, \gamma \in \mathbb{N}^{n}\right.$ e $\left.|\gamma| \leq|\alpha|+1\right\} \leq \bar{c} \varepsilon^{-\bar{N}}$

Portanto para todo $\varepsilon \in] 0, \eta_{3}[$ temos, por (2), (4) e (5), que

e, de (2) e (6), que

$$
\sup \left\{\frac{1}{|J \widehat{f}(\varepsilon, \hat{g}(\varepsilon, y))|}: y \in K^{\prime}\right\} \leq \frac{1}{\mu(\varepsilon)} \leq \bar{c} \varepsilon^{-\bar{N}}
$$

$$
\sup \left\{\left|\partial^{\gamma} \widehat{f}_{i}(\varepsilon, \hat{g}(\varepsilon, y))\right|: y \in K^{\prime}, 1 \leq i \leq n, \gamma \in \mathbb{N}^{n} \text { e }|\gamma| \leq|\alpha|+1\right\} \leq \bar{c} \varepsilon^{-\bar{N}} .
$$

Utilizando (3), (7) e (8) para $|\gamma|=1$, concluímos que (1) é verdadeira se $|\alpha|=1$.

Suponhamos $|\alpha|>1$ e seja $\nu \in \mathbb{N}^{n} \operatorname{com}|\nu|=|\alpha|+1$.

Provaremos que (1) é verdadeira para $\nu$, admitindo, por hipótese de indução, que a afirmação (1) é verdadeira com $\beta$ no lugar de $\alpha$, sendo $\beta \in \mathbb{N}^{n}$ e $|\beta| \leq|\nu|-1=|\alpha|$.

Pela hipótese de indução, existem $N_{1} \in \mathbb{N}, c_{1}>0$ e $\left.\eta_{4} \in\right] 0, \eta_{3}[$ tais que

$$
\sup \left\{\left|\partial^{\beta} \widehat{g}_{l}(\varepsilon, y)\right|: y \in K^{\prime}, 1 \leq l \leq n, \beta \in \mathbb{N}^{n} \mathrm{e}|\beta| \leq|\alpha|\right\} \leq c_{1} \varepsilon^{-N_{1}}
$$

Para $k \in\{1,2, \ldots, n\}$ existem $j \in\{1,2, \ldots, n\}$ e $\tilde{\gamma} \in \mathbb{N}^{n}$ com $|\tilde{\gamma}|=|\alpha|$ tais que

$$
\partial^{\nu} \widehat{g}_{k}(\varepsilon, y)=\partial^{\tilde{\gamma}} \frac{\partial \widehat{g}_{k}}{\partial y_{j}}(\varepsilon, y),
$$


e assim, de (3), temos que

$$
\partial^{\nu} \widehat{g}_{k}(\varepsilon, y)=\partial^{\dot{\gamma}}\left(\frac{1}{J \widehat{f}(\varepsilon, \widehat{g}(\varepsilon, y))} a_{j k}\right) .
$$

Desenvolvendo o segundo membro de (10) pela fórmula de Leibiniz, observando que $\partial^{\bar{\gamma}}\left(\frac{1}{J \widehat{f}(\varepsilon, \widehat{g}(\varepsilon, y)}\right)$ é uma soma de termos do tipo $r(J \widehat{f}(\varepsilon, \widehat{g}(\varepsilon, y)))^{-p} b(\varepsilon, y)$, onde $r$ é um número inteiro, $p \in \mathbb{N}^{*}$ e $b(\varepsilon, y)$ é soma de produtos de elementos da reunião dos conjuntos

$$
\begin{gathered}
\left\{\left|\partial^{\gamma} \widehat{f}_{i}(\varepsilon, \hat{g}(\varepsilon, y))\right|: \gamma \in \mathbb{N}^{n} \text { com }|\gamma| \leq|\alpha|+1 \text { e } 1 \leq i \leq n\right\} \\
\left\{\partial^{\lambda} \hat{g}_{i}(\varepsilon, y): \lambda \in \mathbb{N}^{n} \text { com }|\lambda| \leq|\alpha| \text { e } 1 \leq i \leq n\right\}
\end{gathered}
$$

e usando (7), (8) e (9) para $1 \leq k \leq n$ prova-se que (1) é verdadeira para $\nu$.

Portanto (a) implica (b).

Usando (b), (i), (ii), (iii) e 1.2.9, obtemos (c).

De 1.2.12 concluímos que (c) implica (a).

Portanto (a), (b) e (c) são equivalentes. //

Analisando a prova de que 1.2.15.a implica 1.2.15.b obtivemos os seguintes lemas que nos serão úteis.

1.2.16 Lema. Sejam $\Omega$ e $\Omega^{\prime}$ abertos de $\mathbb{R}^{n}, U$ aberto de $\Omega, V$ aberto de $\Omega^{\prime}$ e $f$, $\widehat{f}=\left(\widehat{f}_{1}, \ldots, \hat{f}_{n}\right), \tau$ e $\hat{g}=\left(\widehat{g}_{1}, \ldots, \widehat{g}_{n}\right)$ como em 1.2.15. Se

(i) $\widehat{g}(] 0, \tau[\times V) \subset U$;

(ii) $\inf \{|J \hat{f}(\varepsilon, x)|:(\varepsilon, x) \in] 0, \tau[\times U\}>0$;

(iii) existe $M>0$ tal que para todo $\alpha \in \mathbb{N}^{n}$ com $|\alpha|=1$ tem-se que

$$
\left.\left|\partial^{\alpha} \hat{f}_{i}(\varepsilon, x)\right| \leq M, \text { para todo }(\varepsilon, x) \in\right] 0, \tau[\times U \text { e } 1 \leq i \leq n
$$

entâo existe $\bar{M}>0$ tal que para todo $\gamma \in \mathbb{N}^{n}$ com $|\gamma|=1$ tem-se que

$$
\left.\left|\partial^{\gamma} \widehat{g}_{i}(\varepsilon, y)\right| \leq \bar{M}, \text { para todo }(\varepsilon, y) \in\right] 0, \tau[\times V \text { e } 1 \leq i \leq n
$$


Demonstração. Basta usar a afirmação (3) da prova de 1.2 .15 e as afirmações (i), (ii) e (iii) deste lema. //

1.2.17 Lema. Sejam $\Omega$ e $\Omega^{\prime}$, abertos de $\mathbb{R}^{n}$ e $f=\left(f_{1}, \ldots, f_{n}\right), \hat{f}, \tau$ e $\hat{g}=\left(\widehat{g}_{1}, \ldots, \widehat{g}_{n}\right)$ como em 1.2.15. Se

(i) $\partial^{\alpha} f_{i} \in \mathcal{G}_{*}\left(\Omega ; \mathbb{R}^{n}\right), \quad$ para todo $\alpha \in \mathbb{N}^{n}$ com $|\alpha|=1 \quad$ e $1 \leq i \leq n$;

(ii) dado qualquer $K \subset \subset \Omega$ tem-se $\inf \{|J \widehat{f}(\varepsilon, x)|:(\varepsilon, x) \in] 0, \tau[\times K\}>0$, entâo $\hat{g} \in \mathcal{E}_{M}\left[\Omega^{\prime} ; \mathbb{R}^{n}\right]$ e, se $g=\left(g_{1}, \ldots, g_{n}\right)$ é a classe de $\hat{g}$ em $\mathcal{G}\left(\Omega^{\prime} ; \mathbb{R}^{n}\right)$ tem-se que

$$
\partial^{\gamma} g_{i} \in \mathcal{G}_{*}\left(\Omega^{\prime} ; \mathbb{R}\right), \quad \text { para todo } \gamma \in \mathbb{N}^{n} \text { com }|\gamma|=1 \text { e } 1 \leq i \leq n
$$

Demonstração. Usando 1.1.32 temos que 1.2.15.a é verdadeira e portanto $\hat{g} \in \mathcal{E}_{M}\left[\Omega^{\prime} ; \mathbb{R}^{n}\right]$ (1.2.15). Para concluir a prova dado qualquer $K^{\prime} \subset \subset \Omega^{\prime}$ basta usar as afirrnações (2) e (3) da demonstração de 1.2 .15 e as asserções (i) e (ii), deste lema, para o compacto de $\Omega$ que foi determinado em (2). //

Na proposição abaixo utilizaremos 1.2 .15 para provar que uma certa função generalizada é inversível.

1.2.18 Proposição. Sejam h uma função definida em ]0,1] e com valores em $\mathbb{R}$ tal que $h(] 0,1]) \subset\left[\frac{1}{2}, 1\right]$ e $\widehat{f}$ a função definida em $\left.\left.] 0,1\right] \times\right] 0,1[$ por

$$
\widehat{f}(\varepsilon, x)=\operatorname{sen}\left(x^{h(\varepsilon)}\right)+x .
$$

\section{Então}

(I) $\widehat{f} \in \mathcal{E}_{M}[] 0,1[; \mathbb{R}]$;

(II) se $f$ é a classe da função $\widehat{f}$ em $\mathcal{G}(] 0,1[; \mathbb{R})$, então $f \in \mathcal{G}_{*}(] 0,1[;] 0, \operatorname{sen}(1)+1[)$ e $f$ é uma funçâo inversível. 
Demonstração. A primeira asserção é de fácil verificação. Provaremos a seguir (II).

Seja $f$ a classe da função $\hat{f}$ em $\mathcal{G}(] 0,1[; \mathbb{R})$. Tomemos $K \subset \subset] 0,1[$ e sejam $a$ e $b$ números reais tais que $K \subset[a, b] \subset] 0,1[$. Então

$$
0<a+\operatorname{sen} a \leq x+\operatorname{sen} x \leq x+\operatorname{sen}\left(x^{h(\varepsilon)}\right) \leq x+\operatorname{sen} 1 \leq b+\operatorname{sen} 1<1+\operatorname{sen} 1
$$

para todo $x \in K$ e $\varepsilon \in] 0,1]$.

Portanto

$$
\{\widehat{f}(\varepsilon, x):(\varepsilon, x) \in] 0,1] \times K\} \subset[a+\operatorname{sen} a, b+\operatorname{sen} 1] \subset \subset] 0,1+\operatorname{sen} 1[
$$

$\mathrm{e} \operatorname{assim} f \in \mathcal{G}_{*}(] 0,1[;] 0,1+\operatorname{sen} 1[)$.

Notemos que $J \widehat{f}(\varepsilon, x)=\cos \left(x^{h(\varepsilon)}\right) \frac{h(\varepsilon)}{x^{1-h(\varepsilon)}}+1>1$, e assim $J \widehat{f}(\varepsilon, x)>1$ para todo $(\varepsilon, x) \in] 0,1] \times] 0,1[$. Portanto $J f$ tem inverso multiplicativo em $\mathcal{G}(] 0,1[; \mathbb{R})(1.1 .32)$.

Fixemos $\varepsilon \in] 0,1]$. Como $\widehat{f}(\varepsilon,$.$) é uma função estritamente crecente, \lim _{x \downarrow 0} \widehat{f}(\varepsilon, x)=0$ e $\lim _{x \uparrow 1} \hat{f}(\varepsilon, x)=1+\operatorname{sen} 1$, concluímos que $\hat{f}(\varepsilon,$.$) admite função inversa g_{\varepsilon}$ definida em ] $0,1+\operatorname{sen} 1[$ e com valores em $] 0,1[$.

Sejam $l_{1}$ e $l_{2}$ as funções definidas em ]0,1[ por

$$
l_{1}(x)=x+\operatorname{sen} x \quad \text { e } \quad l_{2}(x)=x+\operatorname{sen} \sqrt{x} .
$$

Então $l_{1}$ e $l_{2}$ são funções estritamente crescentes em $] 0,1\left[, l_{1} \leq \hat{f}(\varepsilon,.) \leq l_{2}\right.$, para todo $\varepsilon \in] 0,1]$ e $\left.l_{1}(] 0,1[)=l_{2}(] 0,1[)=\right] 0,1+\operatorname{sen}(1)[$.

Seja $\left.K^{\prime} \subset \subset\right] 0,1+\operatorname{sen} 1\left[\right.$ e tomemos $c$ e $d$ números reais tais que $K^{\prime} \subset[c, d] \subset$ ] $0,1+\operatorname{sen} 1[$. Então, para todo $\varepsilon \in] 0,1]$ temos que

$$
\begin{aligned}
\left\{g_{\varepsilon}(y): y \in K^{\prime}\right\} & \subset\{x \in] 0,1\left[: \hat{f}(\varepsilon, x) \in K^{\prime}\right\} \\
& \subset\{x \in] 0,1[: c \leq \widehat{f}(\varepsilon, x) \leq d\} \\
& \left.\left.\subset l_{1}^{-1}(]-\infty, d\right]\right) \cap l_{2}^{-1}([c, \infty[) .
\end{aligned}
$$

Sejam $x_{1}$ e $x_{2}$ pertencentes a ]0,1[ tais que $l_{1}\left(x_{1}\right)=d$ e $l_{2}\left(x_{2}\right)=c$. Usando que $l_{1}$ e $l_{2}$ são funções estritamente crescentes temos que

$$
\left.\left.\left.\left.l_{1}^{-1}(]-\infty, d\right]\right) \subset\right] 0, x_{1}\right] \quad \text { e } \quad l_{2}^{-1}\left(\left[c, \infty[) \subset\left[x_{2}, 1[,\right.\right.\right.
$$


e como $l_{1} \leq l_{2}$ e $c \leq d$ temos que $x_{2} \leq x_{1}$.

Portanto

$$
\left.\left.\left.\left\{g_{\varepsilon}(y):(\varepsilon, y) \in\right] 0,1\right] \times K^{\prime}\right\} \subset\left[x_{2}, x_{1}\right] \subset \subset\right] 0,1[.
$$

Usando 1.2.15 concluímos que $f$ é uma função inversível. //

Completamos o resultado acima exibindo funções $h$ verificando as hipóteses do mesmo.

1.2.19 Exemplo. Em 1.2.18 podemos substituir a função $h$, por exemplo, pela função cosseno, ou pela função $h_{1}(\varepsilon)=\frac{\sqrt{\varepsilon+1}}{2}$ ou ainda pela função

$$
h_{2}(\varepsilon)=\left\{\begin{array}{cc}
\frac{\operatorname{sen} \varepsilon}{\varepsilon} & , \text { se } \varepsilon \in] 0, \tau[ \\
1 & , \text { se } \varepsilon \in[\tau, 1]
\end{array},\right.
$$

onde $\tau \in] 0,1]$ é tal que $\frac{\operatorname{sen} \varepsilon}{\varepsilon}>\frac{1}{2}$, para todo $\left.\varepsilon \in\right] 0, \tau[$.

Quanto ao problema proposto no início desta seção, usando os teoremas 1.2.9 e 1.2.15, concluímos o seguinte:

Resposta: Se $\Omega$ e $\Omega^{\prime}$ são abertos de $\left.\left.\mathbb{R}^{n}, f \in \mathcal{G}_{*}\left(\Omega ; \Omega^{\prime}\right), \tau \in\right] 0,1\right]$ e $\hat{f}$ é um representante de $f$ satisfazendo:

(i) $\{\widehat{f}(\varepsilon, x): x \in \Omega\}=\Omega^{\prime}$, para todo $\left.\varepsilon \in\right] 0, \tau[$;

(ii) $\widehat{f}(\varepsilon,$.$) é uma aplicação inversível com aplicação inversa g_{\varepsilon} \in \mathcal{C}^{\infty}\left(\Omega^{\prime} ; \Omega\right)$, para todo $\varepsilon \in] 0, \tau[$,

e se $\widehat{g}$ é uma aplicação definida em $] 0,1] \times \Omega^{\prime}$ por $\hat{g}(\varepsilon,)=.g_{\varepsilon}$ para todo $\left.\varepsilon \in\right] 0, \tau[e$ $\widehat{g}(\varepsilon,)=.g_{\frac{\tau}{2}}$ para todo $\varepsilon \in[\tau, 1]$, temos que

(I) se $\hat{g} \in \mathcal{E}_{M}\left[\Omega^{\prime} ; \mathbb{R}^{n}\right]$ (1.2.9.iii), então $f$ é uma aplicação inversível se, e somente se, g satisfaz 1.2.15.iii;

(II) se $\widehat{g}$ satisfaz 1.2.15.iii, então $f$ é uma aplicação inversivel se, e somente se, $\widehat{g} \in$ $\mathcal{E}_{M}\left[\Omega^{\prime} ; \mathbb{R}^{n}\right]$. 
Não temos resposta, até o momento, para o caso em que $\widehat{g} \notin \mathcal{E}_{M}\left[\Omega^{\prime} ; \mathbb{R}^{n}\right]$ e não satisfaz 1.2.15.iii.

Uma maneira de resolver as equações de Hamilton-Jacobi, e que será utilizado no capítulo 2, é usar o método das características. Quando usamos este método precisamos determinar a aplicação inversa de uma função $Y \in \mathcal{G}_{*}(I \times \Omega ; W)$ que tem um representante da forma $\widehat{Y}(\varepsilon, t, x)=(t, \hat{g}(\varepsilon, t, x)+x)$, para todo $(\varepsilon, t, x) \in] 0,1] \times I \times \Omega$, sendo $I$ um intervalo aberto de $\mathbb{R}$ com $0 \in I, \Omega$ aberto de $\mathbb{R}^{n}$ e $W$ aberto de $\mathbb{R}^{n+1}$. Por essa razão passaremos a estudar esse tipo de funções. Antes porém apresentaremos um resultado que nos será útil.

1.2.20 Proposição. Sejam $\left.t_{o} \in \mathbb{R}, r>0, I=\right] t_{o}-r, t_{o}+r\left[, \Omega\right.$ um aberto de $\mathbb{R}^{n}, \Omega^{\prime}$ um aberto de $\mathbb{R}^{n+1}$ e $f \in \mathcal{G}_{*}\left(I \times \Omega ; \Omega^{\prime}\right)$. Indicando por $(t, x)=\left(t, x_{1}, \ldots, x_{n}\right)$ um ponto genérico de $I \times \Omega$ tem-se que, se existem $\hat{f}=\left(\hat{f}_{1}, \hat{f}_{2}, \ldots, \hat{f}_{n+1}\right)$ um representante de $f e$ $\tau \in] 0,1]$ satisfazendo:

(i) existem $\bar{M}>0$ e $\eta \in] 0, \tau\left[\right.$ tais que, para todo $\alpha=\left(\alpha_{0}, \alpha_{1}, \ldots, \alpha_{n}\right) \in \mathbb{N}^{n+1}$ com $|\alpha|=1$ ou $0<\alpha_{0} \leq|\alpha|=2$ e $1 \leq i \leq n+1$ tem-se $r \bar{M}<\inf \left\{\left|J \widehat{f}\left(\varepsilon, t_{o}, x\right)\right|:(\varepsilon, x) \in\right] 0, \eta[\times \Omega\}$
$\sup \left\{\left|\partial^{\alpha} \widehat{f}_{i}(\varepsilon, t, x)\right|:(\varepsilon, t, x) \in\right] 0, \eta[\times I \times \Omega\} \leq\left[\frac{\bar{M}}{(n+1)(n+1) !}\right]^{\frac{1}{n+1}}$

(ii) $\{\widehat{f}(\varepsilon, t, x):(t, x) \in I \times \Omega\}=\Omega^{\prime}$, para todo $\left.\varepsilon \in\right] 0, \tau[$;

(iii) $\widehat{f}(\varepsilon,$.$) é uma aplicação inversível com aplicação inversa g_{\varepsilon}$, para todo $\left.\varepsilon \in\right] 0, \tau[$;

(iv) dado qualquer $K^{\prime} \subset \subset \Omega^{\prime}$, existem $K \subset \subset I \times \Omega$ e $\left.\eta_{1} \in\right] 0, \tau[$ tais que

$$
\left\{g_{\varepsilon}(y): y \in K^{\prime} \text { e } \varepsilon \in\right] 0, \eta_{1}[\} \subset K ;
$$

então

(I) $\inf \{|J \widehat{f}(\varepsilon, t, x)|:(\varepsilon, t, x) \in] 0, \eta[\times I \times \Omega\}>0$;

(II) $f$ é uma aplicação inversivel; 
(III) a aplicação moderada $\hat{g}=\left(\widehat{g}_{1}, \ldots, \hat{g}_{n+1}\right)$ definida em $\left.] 0,1\right] \times \Omega^{\prime}$ por $\hat{g}(\varepsilon,)=.g_{\varepsilon}$ para todo $\varepsilon \in] 0, \tau\left[\right.$ e $\hat{g}(\varepsilon,)=.g_{\frac{\tau}{2}}$ para todo $\varepsilon \in[\tau, 1]$, é um representante de $f^{-1}$ e existe $M>0$ tal que $\left|\partial^{\gamma} \widehat{g}_{i}(\varepsilon, y)\right| \leq M$, para todo $\gamma \in \mathbb{N}^{n+1}$ com $|\gamma|=1$, $(\varepsilon, y) \in] 0, \tau\left[\times \Omega^{\prime} \quad\right.$ e $1 \leq i \leq n+1$.

Demonstração. Usando (i) e 1.1.33 obtemos (I) e que $J f$ tem inverso multiplicativo em $\mathcal{G}(I \times \Omega ; \mathbb{R})$, e portanto (II) e (III) são verdadeiras $(1.2 .15$ e 1.2 .16$)$. //

A partir daqui utilizaremos a seguinte notação:

1.2.21 Notação. Seja $a>0$. Denotaremos por $I_{a}$ e por $\overline{I_{a}}$, os intervalos da reta ]$-a, a[e[-a, a]$ respectivamente.

Utilizando 1.2.20 obtemos:

1.2.22 Proposição. Sejam $I$ um intervalo aberto de $\mathbb{R}$ com $0 \in I, \tau \in] 0,1]$ e $\hat{g}=$ $\left(\widehat{g}_{1}, \ldots, \widehat{g}_{n}\right) \in \mathcal{E}_{M}\left[I \times \mathbb{R}^{n} ; \mathbb{R}^{n}\right]$. Denotando por $(t, x)=\left(t, x_{1}, \ldots, x_{n}\right)$ um ponto genérico de $I \times \mathbb{R}^{n}$ tem-se que, se $f$ é a classe, em $\mathcal{G}\left(I \times \mathbb{R}^{n} ; \mathbb{R}^{n+1}\right)$, da aplicação moderada definida em $] 0,1] \times I \times \mathbb{R}^{n}$ por

$$
\widehat{f}(\varepsilon, t, x)=(t, \widehat{g}(\varepsilon, t, x)+x)
$$

e $\hat{g}$ satisfaz

(i) $\hat{g}_{i}(\varepsilon, 0, x)=0, \quad$ para todo $\left.(\varepsilon, x) \in\right] 0, \tau\left[\times \mathbb{R}^{n}\right.$ e $1 \leq i \leq n$;

(ii) existe $M>0$ tal que

$$
\max \left\{\left|\frac{\partial \widehat{g}_{i}}{\partial t}(\varepsilon, t, x)\right|,\left|\frac{\partial^{2} \widehat{g}_{i}}{\partial t^{2}}(\varepsilon, t, x)\right|,\left|\frac{\partial^{2} \widehat{g}_{i}}{\partial x_{j} \partial t}(\varepsilon, t, x)\right|\right\} \leq M,
$$

para todo $(\varepsilon, t, x) \in] 0, \tau\left[\times I \times \mathbb{R}^{n} \quad\right.$ e $1 \leq i, j \leq n$,

então existe $a>0$ com $\overline{I_{a}} \subset I$ e tal que

(I) $\left.f\right|_{I_{a} \times R^{n}} \in \mathcal{G}_{*}\left(I_{a} \times \mathbb{R}^{n} ; I_{a} \times \mathbb{R}^{n}\right)$; 
(II) $\left.f\right|_{I_{a} \times R^{n}}$ é uma aplicação inversível;

(III) $\inf \{|J \widehat{f}(\varepsilon, t, x)|:(\varepsilon, t, x) \in] 0, \tau\left[\times I_{a} \times \mathbb{R}^{n}\right\}>0$;

(IV) $\left.\hat{f}(\varepsilon,)\right|_{.I_{a} \times R^{n}}$ é uma aplicação inversível, para todo $\left.\left.\varepsilon \in\right] 0,1\right]$, a aplicação moderada $\hat{\Gamma}=\left(\widehat{\Gamma}_{0}, \hat{\Gamma}_{1}, \ldots, \hat{\Gamma}_{n}\right)$ definida em $\left.] 0,1\right] \times I_{a} \times \mathbb{R}^{n}$ por $\hat{\Gamma}(\varepsilon,)=.\left(\left.\hat{f}(\varepsilon, .)\right|_{I_{a} \times R^{n}}\right)^{-1}$ para todo $\varepsilon \in] 0, \tau\left[\right.$ e $\widehat{\Gamma}(\varepsilon,)=.\left(\left.\widehat{f}\left(\frac{\tau}{2}, .\right)\right|_{I_{a} \times R^{n}}\right)^{-1}$ para todo $\varepsilon \in[\tau, 1]$, é um representante de $\left(\left.f\right|_{I_{a} \times R^{n}}\right)^{-1}$ e existe $\bar{M}>0$ tal que $\left|\partial^{\alpha} \widehat{\Gamma}_{i}(\varepsilon, t, y)\right| \leq \bar{M}$, para todo $(\varepsilon, t, y) \in] 0, \tau\left[\times I_{a} \times \mathbb{R}^{n}, \alpha \in \mathbb{N}^{n+1} \quad\right.$ com $|\alpha|=1$ e $0 \leq i \leq n$.

Demonstração. Sejam $M$ como em (ii), $a^{*}>0$ com $\overline{I_{a^{*}}} \subset I$ e $a>0$ tal que

$$
a<\min \left\{a^{*}, \frac{1}{M n}, \frac{1}{(n+1)(n+1) ! M^{n+1}}, \frac{n^{n+1}}{(n+1) !(n+1)^{n+2}}\right\} .
$$

Notemos que, se $\varepsilon \in] 0, \tau\left[, 1 \leq i, j \leq n\right.$ e $(t, x) \in I \times \mathbb{R}^{n}$, então, usando o Teorema do Valor Médio, existem $t_{1}$ e $t_{2}$ entre 0 e $t$ tais que

$$
\begin{aligned}
& \widehat{g}_{i}(\varepsilon, t, x)-\widehat{g}_{i}(\varepsilon, 0, x)=\frac{\partial \widehat{g}_{i}}{\partial t}\left(\varepsilon, t_{1}, x\right) t ; \\
& \frac{\partial \widehat{g}_{i}}{\partial x_{j}}(\varepsilon, t, x)-\frac{\partial \widehat{g}_{i}}{\partial x_{j}}(\varepsilon, 0, x)=\frac{\partial^{2} \widehat{g}_{i}}{\partial t \partial x_{j}}\left(\varepsilon, t_{2}, x\right) t,
\end{aligned}
$$

e assim, usando (i) e (ii), temos que

$$
\begin{aligned}
& \left|\widehat{g}_{i}(\varepsilon, t, x)\right| \leq M|t| ; \\
& \left|\frac{\partial \widehat{g}_{i}}{\partial x_{j}}(\varepsilon, t, x)\right| \leq M|t| .
\end{aligned}
$$

Seja $\varphi$ a função contínua definida em $I_{a} \times \mathbb{R}^{n}$ por $\varphi(t, x)=n M|t|+\|x\|$.

Provaremos, em primeiro lugar, a asserção (I).

Seja $K^{\prime} \subset \subset I_{a} \times \mathbb{R}^{n}$ e tomemos $L \subset \subset I_{a}$ e $K \subset \subset \mathbb{R}^{n}$ tais que $K^{\prime} \subset L \times K$. Então, por (1), se $r=\max \{\varphi(s, y):(s, y) \in L \times K\}$ temos

$$
\|\widehat{g}(\varepsilon, t, x)+x\| \leq\|\hat{g}(\varepsilon, t, x)\|+\|x\| \leq n M|t|+\|x\|=\varphi(t, x) \leq r
$$

para todo $(\varepsilon, t, x) \in] 0, \tau[\times L \times K$.

Portanto

$$
\left\{\widehat{f}(\varepsilon, t, x):(t, x) \in K^{\prime} \text { e } \varepsilon \in\right] 0, \tau[\} \subset L \times \overline{B_{r}(0)} \subset \subset I_{a} \times \mathbb{R}^{n}
$$


o que prova (I).

Para provarmos (II), (III) e (IV) utilizaremos 1.2.20.

Fixemos $\varepsilon \in] 0, \tau\left[\right.$. Provaremos que $\left.\hat{f}(\varepsilon,)\right|_{.I_{a} \times R^{n}}$ é uma aplicação bijetora, e assim 1.2.20.ii e 1.2.20.iii estão satisfeitas.

Sejam $(s, y) \in I_{a} \times \mathbb{R}^{n}, d=|| y \|+n M|s|$ e $\quad \psi$ a aplicação definida em $\overline{B_{d}(0)} \subset$ $\mathbb{R}^{n}$ por $\psi(x)=y-\hat{g}(\varepsilon, s, x)$. Então, usando (1), temos

$$
\|\psi(x)\| \leq\|y\|+\|\widehat{g}(\varepsilon, s, x)\| \leq\|y\|+n M|s|=d,
$$

para todo $x \in \overline{B_{d}(0)}$, e assim

$$
\psi\left(\overline{B_{d}(0)}\right) \subset \overline{B_{d}(0)} .
$$

Como $\psi$ é contínua e vale (3) temos, pelo Teorema de Brouwer ([11]), que existe um $\bar{x} \in \overline{B_{d}(0)}$ tal que $\psi(\bar{x})=\bar{x}$, e assim

$$
\hat{f}(\varepsilon, s, \bar{x})=(s, \hat{g}(\varepsilon, s, \bar{x})+\bar{x})=(s, \widehat{g}(\varepsilon, s, \bar{x})+\psi(\bar{x}))=(s, y) .
$$

Portanto $\left.\hat{f}(\varepsilon,)\right|_{.I_{a} \times R^{n}}$ é uma função sobrejetora.

Provaremos, a seguir, que $\left.\hat{f}(\varepsilon,)\right|_{.I_{a} \times R^{n}}$ é uma função injetora.

Sejam $(s, x)$ e $(t, y)$ pertencentes a $I_{a} \times \mathbb{R}^{n}$ e diferentes.

Se $s \neq t$ é claro que $\hat{f}(\varepsilon, s, x) \neq \widehat{f}(\varepsilon, t, y)$.

Suponhamos $s=t$ e seja $j \in\{1, \ldots, n\}$ tal que

$$
0<\max \left\{\left|x_{i}-y_{i}\right|: 1 \leq i \leq n\right\}=\left|x_{j}-y_{j}\right|
$$

Então, usando o Teorema do Valor Médio, existe $z$ no segmento de extremidades $x$ e $y$ tal que

$$
\widehat{g}_{j}(\varepsilon, s, x)+x_{j}-\widehat{g}_{j}(\varepsilon, s, y)-y_{j}=\sum_{i=1}^{n} \frac{\partial \widehat{g}_{j}}{\partial x_{i}}(\varepsilon, s, z)\left(x_{i}-y_{i}\right)+x_{j}-y_{j},
$$

e assim, por (2), temos

$$
\begin{aligned}
\left|\hat{g}_{j}(\varepsilon, s, x)+x_{j}-\hat{g}_{j}(\varepsilon, s, y)-y_{j}\right| & \geq\left|x_{j}-y_{j}\right|-\sum_{i=1}^{n} M|s|\left|x_{i}-y_{i}\right| \\
& \geq\left|x_{j}-y_{j}\right|-n M a\left|x_{j}-y_{j}\right| \\
& \geq\left|x_{j}-y_{j}\right|(1-n M a)>0,
\end{aligned}
$$


donde concluimos que $\hat{f}(\varepsilon, s, x) \neq \widehat{f}(\varepsilon, s, y)$.

Portanto $\left.\hat{f}(\varepsilon,)\right|_{.I_{a} \times R^{n}}$ é uma função injetora.

Provaremos agora que as asserções 1.2.20.i e 1.2.20.iv são verdadeiras.

Seja $\widehat{f}_{j}$, para $0 \leq j \leq n$, a função definida em $\left.] 0,1\right] \times I_{a} \times \mathbb{R}^{n}$ por

$$
\widehat{f}_{j}(\varepsilon, s, x)=s \text { se } j=0 \quad \text { e } \quad \hat{f}_{j}(\varepsilon, s, x)=\hat{g}_{j}(\varepsilon, s, x)+x_{j} \text { se } j \neq 0 .
$$

Notemos que $\hat{f}=\left(\hat{f}_{0}, \hat{f}_{1}, \ldots, \widehat{f}_{n}\right)$ e que, por (i),

$$
|J \hat{f}(\varepsilon, 0, x)|=1, \text { para todo }(\varepsilon, x) \in] 0, \tau\left[\times \mathbb{R}^{n}\right. \text {. }
$$

Sejam $\beta=\left(\beta_{0}, \beta_{1}, \ldots, \beta_{n}\right) \in \mathbb{N}^{n+1}$ e $p=\max \left\{\frac{n+1}{n}, M\right\}$.

Como $(n+1)(n+1) ! p^{n+1}<\frac{1}{a}$ existe $\bar{M}>0$ tal que $(n+1)(n+1) ! p^{n+1}<\bar{M}<\frac{1}{a}$, e assim se $|\beta|=1$ ou $0<\beta_{0} \leq|\beta|=2$ temos, por (ii) e (2), que

$$
\left|\partial^{\beta} \widehat{f}_{j}(\varepsilon, s, x)\right| \leq \max \{1+M a, M\}<\max \left\{\frac{n+1}{n}, M\right\}=p<\left(\frac{\bar{M}}{(n+1)(n+1) !}\right)^{\frac{1}{n+1}},
$$

para todo $0 \leq j \leq n$ e $(\varepsilon, s, x) \in] 0, \tau\left[\times I_{a} \times \mathbb{R}^{n}\right.$, o que prova a segunda desigualdade de 1.2.20.i

Notemos também que

$$
a \bar{M}<1=\inf \left\{|J \hat{f}(\varepsilon, 0, x)|: x \in \mathbb{R}^{n} \text { e } \varepsilon \in\right] 0, \tau[\}
$$

o que prova a primeira desigualdade de 1.2.20.i.

Provaremos agora que vale 1.2.20.iv.

Sejam $K^{\prime} \subset \subset I_{a} \times \mathbb{R}^{n}$ e tomemos $L \subset \subset I_{a}$ e $K \subset \subset \mathbb{R}^{n}$ tais que $K^{\prime} \subset L \times K$.

Fixemos $\varepsilon \in] 0, \tau\left[\right.$ e seja $h_{\varepsilon}$ a aplicação inversa de $\left.\hat{f}(\varepsilon,)\right|_{.I_{a} \times R^{n}}$. Então para todo $(s, y) \in K^{\prime}$ temos que $h_{\varepsilon}(s, y)=(s, x)$ onde $\hat{f}(\varepsilon, s, x)=(s, \widehat{g}(\varepsilon, s, x)+x)=(s, y)$, e assim, usando (1), obtemos

$$
\begin{aligned}
\|x\| & \leq\|x+\hat{g}(\varepsilon, s, x)\|+\|\widehat{g}(\varepsilon, s, x)\| \\
& \leq\|y\|+n M|s| \\
& \leq \sup \{\|z\|: z \in K\}+n M a .
\end{aligned}
$$


Portanto, se $r_{1}=\sup \{\|z\|: z \in K\}+n M a$, temos que

$$
\left\{h_{\varepsilon}(s, y):(s, y) \in K^{\prime} \text { e } \varepsilon \in\right] 0, \tau[\} \subset L \times \overline{B_{r_{1}}(0)} \subset \subset I_{a} \times \mathbb{R}^{n}
$$

Portanto 1.2.20.iv é verdadeira, e assim concluímos (II), (III) e (IV). //

Completamos o resultado acima exibindo uma função $\hat{g}$ que verifica as hipóteses do mesmo.

1.2.23 Exemplo. Sejam $h$ uma função definida em ]0,1] e com valores em $\mathbb{R}$ e $\varphi$ e $\psi$ pertencentes a $\mathcal{C}^{\infty}(\mathbb{R} ; \mathbb{R})$ tais que $\psi(0)=0$ e $h, \varphi, \psi, \varphi^{\prime}, \psi^{\prime}$ e $\psi^{\prime \prime}$ são funções limitadas. Se $b>0$ e $\hat{g}=\left(\widehat{g}_{1}, \ldots, \widehat{g}_{n}\right) \in \mathcal{E}_{M}\left[I_{b} \times \mathbb{R}^{n} ; \mathbb{R}^{n}\right]$ é tal que $\hat{g}_{i}$ é uma das funções

$$
\begin{gathered}
\hat{l}_{1}\left(\varepsilon, t, x_{1}, \ldots, x_{n}\right)=t \varphi\left(h(\varepsilon) \sum_{j=1}^{n} x_{j}\right) ; \\
\hat{l}_{2}\left(\varepsilon, t, x_{1}, \ldots, x_{n}\right)=\psi\left(t \varphi\left(h(\varepsilon) x_{j}\right)\right), \text { sendo } 1 \leq j \leq n,
\end{gathered}
$$

para todo $1 \leq i \leq n$, então $\hat{g}$ satisfaz as hipóteses de 1.2 .22 .

(Podemos, por exemplo, usar as funções $\Phi_{1}(x)=\operatorname{sen} x, \Phi_{2}(x)=\operatorname{arctg} x$ para $\varphi$ ou $\psi$, e a função $\Phi_{3}(x)=\cos x$ para $\varphi$.)

Em 1.2.22 as componentes da aplicação $\widehat{g}$ pertencem a $\mathcal{E}_{M}\left[I \times \mathbb{R}^{n} ; \mathbb{R}\right]$. Se as componentes de $\widehat{g}$ pertencem a $\mathcal{E}_{M}[I \times \mathbb{R} ; \mathbb{R}]$ obtemos:

1.2.24 Proposição. Sejam I um intervalo aberto de $\mathbb{R}, \tau \in] 0,1]$ e $\hat{g}_{i} \in \mathcal{E}_{M}[I \times \mathbb{R} ; \mathbb{R}]$, para todo $1 \leq i \leq n$. Denotando por $(t, y)$ um ponto genérico de $I \times \mathbb{R}$ tem-se que, se

(i) existem $l \in \mathcal{C}(I ; \mathbb{R})$ e $\left(a_{1}, \ldots, a_{n}\right) \in \mathbb{R}^{n}$ tais que

$$
\left.\left|\widehat{g}_{i}\left(\varepsilon, t, a_{i}\right)\right| \leq l(t), \text { para todo }(\varepsilon, t) \in\right] 0, \tau[\times I \text { e } 1 \leq i \leq n
$$

(ii) existem $l_{1} \in \mathcal{C}(I ; \mathbb{R})$ e $l_{2} \in \mathcal{C}(I ; \mathbb{R})$ tais que $l_{1}^{-1}(]-1, \infty[) \neq \emptyset$ e

$$
\left.l_{1}(t) \leq \frac{\partial \widehat{g}_{i}}{\partial y}(\varepsilon, t, y) \leq l_{2}(t), \text { para todo }(\varepsilon, t, y) \in\right] 0, \tau[\times I \times \mathbb{R} \text { e } 1 \leq i \leq n,
$$


e se $J$ é um intervalo aberto de $\mathbb{R}$ com $J \subset l_{1}^{-1}(]-1, \infty[) \subset I, \hat{f}$ é a aplicação moderada definida em $] 0,1] \times J \times \mathbb{R}^{n}$ e com valores em $\mathbb{R}^{n+1}$ dada por

$$
\widehat{f}\left(\varepsilon, t, x_{1}, \ldots, x_{n}\right)=\left(t, \widehat{g}_{1}\left(\varepsilon, t, x_{1}\right)+x_{1}, \ldots, \widehat{g}_{n}\left(\varepsilon, t, x_{n}\right)+x_{n}\right)
$$

e fé a classe de $\hat{f}$ em $\mathcal{G}\left(J \times \mathbb{R}^{n} ; \mathbb{R}^{n+1}\right)$, então

(I) $f \in \mathcal{G}_{*}\left(J \times \mathbb{R}^{n} ; J \times \mathbb{R}^{n}\right)$;

(II) $f$ é uma aplicação inversivel;

(III) dado qualquer $K^{\prime} \subset \subset J \times \mathbb{R}^{n}$ tem-se

$$
\inf \left\{J \hat{f}(\varepsilon, s, x):(s, x) \in K^{\prime} \text { e } \varepsilon \in\right] 0, \tau[\}>0 ;
$$

(IV) $\hat{f}(\varepsilon,$.$) é uma aplicação inversível para todo \varepsilon \in] 0,1]$, a aplicação moderada $\widehat{\Gamma}$ definida em $] 0,1] \times J \times \mathbb{R}^{n}$ por $\widehat{\Gamma}(\varepsilon,)=.(\widehat{f}(\varepsilon, .))^{-1}$ para todo $\left.\varepsilon \in\right] 0, \tau[e$ $\widehat{\Gamma}(\varepsilon,)=.\left(\widehat{f}\left(\frac{\tau}{2}, .\right)\right)^{-1}$ para todo $\varepsilon \in[\tau, 1]$, é um representante de $f^{-1}$.

Demonstração. Sejam $l, l_{1}, l_{2}$ e $\left(a_{1}, \ldots, a_{n}\right)$ como em (i) e (ii). Provaremos, em primeiro lugar, que (I) é verdadeira.

Seja $K^{\prime} \subset \subset J \times \mathbb{R}^{n}$ e tomemos $L \subset \subset J$ tal que $K^{\prime} \subset L \times \mathbb{R}^{n}$.

Fixemos $\varepsilon \in] 0, \tau\left[\right.$ e $i \in\{1, \ldots, n\}$, e seja $\left(s, x_{1}, \ldots, x_{n}\right) \in K^{\prime}$. Então usando o Teorema do Valor Médio, existe $\overline{\omega_{i}}$ entre $x_{i}$ e $a_{i}$ tal que

$$
\widehat{g}_{i}\left(\varepsilon, s, x_{i}\right)+x_{i}=\widehat{g}_{i}\left(\varepsilon, s, a_{i}\right)+\frac{\partial \widehat{g}_{i}}{\partial y}\left(\varepsilon, s, \overline{\omega_{i}}\right)\left(x_{i}-a_{i}\right)+x_{i}
$$

e assim, por (i) e (ii), temos

$$
\left|\widehat{g}_{i}\left(\varepsilon, s, x_{i}\right)+x_{i}\right| \leq l(s)+\left(\left|l_{1}(s)\right|+\left|l_{2}(s)\right|\right)\left|x_{i}-a_{i}\right|+\left|x_{i}\right|
$$

Sejam $x_{o}=\left(a_{1}, \ldots, a_{n}\right)$ e $\varphi$ a aplicação definida em $I \times \mathbb{R}^{n}$ por

$$
\varphi(t, y)=l(t)+\left(\left|l_{1}(t)\right|+\left|l_{2}(t)\right|\right)\left\|y-x_{o}||+\right\| y \|
$$


que é contínua em $I \times \mathbb{R}^{n}$, e seja $r=\sup \left\{\varphi(t, y):(t, y) \in K^{\prime}\right\}$. Então

$$
\begin{aligned}
\left\|\left(\hat{g}_{1}\left(\varepsilon, s, x_{1}\right)+x_{1}, \ldots, \hat{g}_{n}\left(\varepsilon, s, x_{n}\right)+x_{n}\right)\right\| & \leq \sum_{i=1}^{n}\left(l(s)+\left(\left|l_{1}(s)\right|+\left|l_{2}(s)\right|\right)\left|x_{i}-a_{i}\right|+\left|x_{i}\right|\right) \\
& \leq \sum_{i=1}^{n} \varphi\left(s, x_{1}, \ldots, x_{n}\right) \leq r n
\end{aligned}
$$

para todo $\varepsilon \in] 0, \tau\left[\mathrm{e}\left(s, x_{1}, \ldots, x_{n}\right) \in K^{\prime}\right.$, e assim

$$
\left\{\widehat{f}\left(\varepsilon, s, x_{1}, \ldots, x_{n}\right): \varepsilon \in\right] 0, \tau\left[\text { e }\left(s, x_{1}, \ldots, x_{n}\right) \in K^{\prime}\right\} \subset L \times \overline{B_{r n}(0)} \subset \subset J \times \mathbb{R}^{n}
$$

o que prova (I).

Para provarmos (II) e (IV) utilizaremos 1.2 .15 e para (III) utilizaremos (ii).

Provaremos agora que $\hat{f}(\varepsilon,$.$) é uma aplicação bijetora para todo \varepsilon \in] 0, \tau[$, e assim 1.2.15.i e 1.2.15.ii estão satisfeitas.

Fixemos $\varepsilon \in] 0, \tau\left[\right.$ e seja $\left(t, y_{1}, \ldots, y_{n}\right) \in J \times \mathbb{R}^{n}$. Se $j \in\{1, \ldots, n\}$ e $r \in \mathbb{R}$, então, pelo Teorema do Valor Médio, existe $\bar{r}$ entre 0 e $r$ tal que

$$
\widehat{g}_{j}(\varepsilon, t, r)+r=\widehat{g}_{j}(\varepsilon, t, 0)+\frac{\partial \widehat{g}_{j}}{\partial y}(\varepsilon, t, \bar{r}) r+r=r\left(1+\frac{\partial \widehat{g}_{j}}{\partial y}(\varepsilon, t, \bar{r})\right)+\widehat{g}_{j}(\varepsilon, t, 0)
$$

e assim usando (ii) temos que

$$
\begin{aligned}
& \widehat{g}_{j}(\varepsilon, t, r)+r \geq r\left(1+l_{1}(t)\right)+\widehat{g}_{j}(\varepsilon, t, 0), \text { se } r \geq 0 ; \\
& \hat{g}_{j}(\varepsilon, t, r)+r \leq r\left(1+l_{1}(t)\right)+\widehat{g}_{j}(\varepsilon, t, 0), \text { se } r<0,
\end{aligned}
$$

e assim, observando que $1+l_{1}(t)>0$, concluímos que

$$
\lim _{r \uparrow \infty}\left(\widehat{g}_{j}(\varepsilon, t, r)+r\right)=\infty \text { e } \lim _{r \downarrow-\infty}\left(\widehat{g}_{j}(\varepsilon, t, r)+r\right)=-\infty
$$

e como a função $\psi_{j}$ definida por $\psi_{j}(r)=\widehat{g}_{j}(\varepsilon, t, r)+r$ é contínua, existe $x_{j} \in \mathbb{R}$ com $\psi_{j}\left(x_{j}\right)=y_{j}$.

$$
\begin{aligned}
& \text { Portanto }\left(t, x_{1}, \ldots, x_{n}\right) \in J \times \mathbb{R}^{n} \mathrm{e} \\
& \qquad \hat{f}\left(\varepsilon, t, x_{1}, \ldots, x_{n}\right)=\left(t, \psi_{1}\left(x_{1}\right), \ldots, \psi_{n}\left(x_{n}\right)\right)=\left(t, y_{1}, \ldots, y_{n}\right),
\end{aligned}
$$

provando que $\widehat{f}(\varepsilon,$.$) é uma aplicação sobrejetora.$ 
Sejam $\left(s, x_{1}, \ldots, x_{n}\right)$ e $\left(t, y_{1}, \ldots, y_{n}\right)$ pertencentes a $J \times \mathbb{R}^{n}$ e diferentes.

Se $s \neq t$, então $\hat{f}\left(\varepsilon, s, x_{1}, \ldots, x_{n}\right) \neq \hat{f}\left(\varepsilon, t, y_{1}, \ldots, y_{n}\right)$.

Suponhamos $s=t$. Então existe $j \in\{1, \ldots, n\} \operatorname{com} x_{j} \neq y_{j}$ e podemos supor $x_{j}>y_{j}$. Usando o Teorema do Valor Médio e (ii) temos que

$$
\begin{aligned}
\hat{g}_{j}\left(\varepsilon, s, x_{j}\right)+x_{j}-\hat{g}_{j}\left(\varepsilon, s, y_{j}\right)-y_{j} & =\left(\frac{\partial \widehat{g}_{j}}{\partial y}\left(\varepsilon, s, \bar{\omega}_{j}\right)+1\right)\left(x_{j}-y_{j}\right) \\
& \geq\left(l_{1}(s)+1\right)\left(x_{j}-\hat{y}_{j}\right)>0,
\end{aligned}
$$

para algum $\bar{\omega}_{j}$ entre $x_{j}$ e $y_{j}$, e assim $\hat{f}\left(\varepsilon, s, x_{1}, \ldots, x_{n}\right) \neq \hat{f}\left(\varepsilon, s, y_{1}, \ldots, y_{n}\right)$.

Portanto $\hat{f}(\varepsilon,$.$) é uma aplicação injetora.$

Provaremos, a seguir, que valem 1.2.15.iv e 1.2.15.a.

Notemos que

$$
J \widehat{f}\left(\varepsilon, s, x_{1}, \ldots, x_{n}\right)=\prod_{i=1}^{n}\left(\frac{\partial \widehat{g}_{i}}{\partial y}\left(\varepsilon, s, x_{i}\right)+1\right)
$$

e como $\frac{\partial \widehat{g}_{i}}{\partial y}\left(\varepsilon, s, x_{i}\right)+1 \geq l_{1}(s)+1>0$ para todo $\left.\left(\varepsilon, s, x_{i}\right) \in\right] 0, \tau[\times J \times \mathbb{R}$ temos que

$$
\left.J \hat{f}\left(\varepsilon, s, x_{1}, \ldots, x_{n}\right)>0 \text { para todo }\left(\varepsilon, s, x_{1}, \ldots, x_{n}\right) \in\right] 0, \tau\left[\in J \times \mathbb{R}^{n},\right.
$$

e assim 1.2.15.iv é verdadeira.

Tomemos $K^{\prime} \subset \subset J \times \mathbb{R}^{n}$ e seja $L \subset \subset J$ tal que $K^{\prime} \subset L \times \mathbb{R}^{n}$. Então $J \widehat{f}\left(\varepsilon, s, x_{1}, \ldots, x_{n}\right)=\prod_{i=1}^{n}\left(\frac{\partial \widehat{g}_{i}}{\partial y}\left(\varepsilon, s, x_{i}\right)+1\right) \geq \prod_{i=1}^{n}\left(l_{1}(s)+1\right) \geq\left(\min \left\{l_{1}(t): t \in L\right\}+1\right)^{n}>0$ para todo $\varepsilon \in] 0, \tau\left[\right.$ e $\left(s, x_{1}, \ldots, x_{n}\right) \in K^{\prime}$, e assim vale (III) e por 1.1 .32 concluímos que a asserção 1.2.15.a é verdadeira.

Portanto para concluir a prova basta provar que vale 1.2.15.iii.

Seja $K^{\prime} \subset \subset J \times \mathbb{R}^{n}$ e tomemos $L^{\prime} \subset \subset J \operatorname{com} K^{\prime} \subset L^{\prime} \times \mathbb{R}^{n}$.

Fixemos $\varepsilon \in] 0, \tau\left[\right.$ e seja $h_{\varepsilon}$ a aplicação inversa de $\hat{f}(\varepsilon,$.$) . Então para \left(t, y_{1}, \ldots, y_{n}\right) \in K^{\prime}$ temos $h_{\varepsilon}\left(t, y_{1}, \ldots, y_{n}\right)=\left(t, x_{1}, \ldots, x_{n}\right)$ onde

$$
\widehat{f}\left(\varepsilon, t, x_{1}, \ldots, x_{n}\right)=\left(t, \widehat{g}_{1}\left(\varepsilon, t, x_{1}\right)+x_{1}, \ldots, \widehat{g}_{n}\left(\varepsilon, t, x_{n}\right)+x_{n}\right)=\left(t, y_{1}, \ldots, y_{n}\right)
$$


e assim, usando o Teorema do Valor Médio, para todo $i \in\{1, \ldots, n\}$ existe $\overline{\omega_{i}}$ entre $x_{i}$ e $a_{i}$ tal que

$$
x_{i}=y_{i}-\hat{g}_{i}\left(\varepsilon, t, x_{i}\right)=y_{i}-\widehat{g}_{i}\left(\varepsilon, t, a_{i}\right)-\frac{\partial \hat{g}_{i}}{\partial y}\left(\varepsilon, t, \bar{\omega}_{i}\right)\left(x_{i}-a_{i}\right),
$$

e portanto

$$
x_{i}\left(1+\frac{\partial \widehat{g}_{i}}{\partial y}\left(\varepsilon, t, \bar{\omega}_{i}\right)\right)=y_{i}-\widehat{g}_{i}\left(\varepsilon, t, a_{i}\right)+\frac{\partial \widehat{g}_{i}}{\partial y}\left(\varepsilon, t, \bar{\omega}_{i}\right) a_{i} .
$$

Como $1+\frac{\partial \widehat{g}_{i}}{\partial y}\left(\varepsilon, t, \bar{\omega}_{i}\right) \geq 1+l_{1}(t)>0$ temos, por (i) e (ii), que

$$
\left|x_{i}\right| \leq \frac{1}{1+l_{1}(t)}\left(\left|y_{i}\right|+l(t)+\left(\left|l_{1}(t)\right|+\left|l_{2}(t)\right|\right)\left|a_{i}\right|\right) .
$$

Sejam $x_{o}=\left(a_{1}, \ldots, a_{n}\right), b=\max \left\{\|z\|: z \in K^{\prime}\right\}, p=\min \left\{1+l_{1}(s): s \in L^{\prime}\right\}>0$ e $q=\max \left\{l(s)+\left(\left|l_{1}(s)\right|+\left|l_{2}(s)\right|\right)|| x_{o}||: s \in L^{\prime}\right\}$. Então se $d=\frac{n}{p}(b+q)$ temos, por (1), que

$$
\left\{h_{\varepsilon}\left(t, y_{1}, \ldots, y_{n}\right):\left(t, y_{1}, \ldots, y_{n}\right) \in K^{\prime} \text { e } \varepsilon \in\right] 0, \tau[\} \subset L \times \overline{B_{d}(0)} \subset \subset J \times \mathbb{R}^{n}
$$

e assim 1.2.15.iii é verdadeira.

Portanto, por 1.2.15, obtemos (II) e (IV). //

Para completar o resultado acima apresentamos em 1.2.25 funções que podem ser usadas para $\hat{g}_{i}$, sendo $1 \leq i \leq n$.

1.2.25 Exemplo. Seja $h$ uma função definida em ]0,1] e com valores em $\mathbb{R}$ e $\varphi$ e $\psi$ pertencentes a $\mathcal{C}^{\infty}(\mathbb{R} ; \mathbb{R})$ tais que $\varphi(0)=\psi(0)=0$ e $h, \varphi^{\prime}$ e $\psi^{\prime}$ são funçôes limitadas. Entâo as funçôes moderadas definidas em $] 0,1] \times \mathbb{R} \times \mathbb{R}$ por

$$
\widehat{g}_{1}(\varepsilon, t, y)=t \varphi(h(\varepsilon) y) \quad \text { e } \quad \hat{g}_{2}(\varepsilon, t, y)=t \varphi\left(\frac{1}{\varepsilon} \psi(\varepsilon y)\right)
$$

satisfazem 1.2.24.i para $l=0$ e $a_{1}=a_{2}=0$, e verificam 1.2.24.ii para $l_{1}=-l_{2}=-M^{2}|t|$ para todo $t \in \mathbb{R}$, sendo $M \geq 1 \operatorname{com} h(] 0,1]) \cup \varphi^{\prime}(\mathbb{R}) \cup \psi^{\prime}(\mathbb{R}) \subset[-M, M]$.

(As funções $\Phi_{1}(x)=x, \Phi_{2}(x)=\operatorname{sen} x, \Phi_{3}(x)=\operatorname{arctg} x$ e $\Phi_{4}(x)=\int_{0}^{x} \cos \left(t^{2}\right) d t$ podem ser usadas para $\varphi$ ou $\psi$.) 
A seguir apresentamos um resultado para o caso em que $\Omega=\mathbb{R}_{+}^{*}$.

1.2.26 Proposição. Sejam I um intervalo aberto de $\mathbb{R}$ com $0 \in I, l \in \mathcal{G}_{*}\left(\mathbb{R}_{+}^{*} ; \mathbb{R}_{+}^{*}\right), \hat{l}$ um representante de $l$ satisfazendo:

(i) $\left.\hat{l}(] 0,1] \times \mathbb{R}_{+}^{*}\right) \subset \mathbb{R}_{+}^{*}$;

(ii) $\hat{l}^{\prime}(\varepsilon, x) \geq 0, \quad$ para todo $\left.\left.(\varepsilon, x) \in\right] 0,1\right] \times \mathbb{R}_{+}^{*}$;

(iii) existe $g \in \mathcal{C}\left(\mathbb{R}_{+}^{*} ; \mathbb{R}\right)$, função estritamente crescente e tal que

$$
\left.\left.\lim _{x \downarrow 0} g(x)=0 \quad \text { e } 0<\widehat{l}(\varepsilon, x) \leq g(x), \quad \text { para todo }(\varepsilon, x) \in\right] 0,1\right] \times \mathbb{R}_{+}^{*}
$$

e seja $h \in \mathcal{C}^{\infty}\left(I \times \mathbb{R}_{+}^{*} ; \mathbb{R}\right)$ tal que

(iv) $h(t,$.$) é uma função estritamente crescente, para todo t \in I$;

(v) $\lim _{x \downarrow 0} h(t, x)=0, \quad$ para todo $t \in I$;

(vi) dado qualquer $J \subset \subset I$ existe $c>0$ tal que

$$
h(t, x) \leq h(c, x), \quad \text { para todo }(t, x) \in J \times \mathbb{R}_{+}^{*} .
$$

Se $\hat{f}$ é a aplicação moderada definida em $] 0,1] \times I \times \mathbb{R}_{+}^{*}$ por

$$
\widehat{f}(\varepsilon, t, x)=(t, h(t, \widehat{l}(\varepsilon, x))+x)
$$

e f é a classe de $\hat{f}$ em $\mathcal{G}\left(I \times \mathbb{R}_{+}^{*} ; \mathbb{R}^{2}\right)$, então

(I) $f \in \mathcal{G}_{*}\left(I \times \mathbb{R}_{+}^{*} ; I \times \mathbb{R}_{+}^{*}\right)$;

(II) $\left.\inf \{J \hat{f}(\varepsilon, t, x):(\varepsilon, t, x) \in] 0,1] \times I \times \mathbb{R}_{+}^{*}\right\} \geq 1$;

(III) $f$ é uma aplicação inversível;

(IV) $\widehat{f}(\varepsilon,$.$) é uma aplicação inversível, para todo \varepsilon \in] 0,1]$ e a aplicação moderada $\widehat{\Gamma}$ definida em $] 0,1] \times I \times \mathbb{R}_{+}^{*}$ por $\widehat{\Gamma}(\varepsilon,)=.(\widehat{f}(\varepsilon, .))^{-1}$, para todo $\left.\left.\varepsilon \in\right] 0,1\right]$, é um representante de $f^{-1}$.

(Convém observar que a hipótese (i) é usada somente para definir a aplicação $\hat{f}$.) 
Demonstração. Notemos, em primeiro lugar, que usando (iii) temos

$$
\left.\left.\lim _{x \downarrow 0} \widehat{l}(\varepsilon, x)=0, \text { para todo } \varepsilon \in\right] 0,1\right]
$$

e de (ii) e do fato que g é uma função estritamente crescente temos que

$$
\text { existem } \left.\left.\lim _{x \uparrow \infty} \widehat{l}(\varepsilon, x) \text { e } \lim _{x \uparrow \infty} g(x), \text { para todo } \varepsilon \in\right] 0,1\right],
$$

sendo esses limites finitos ou não.

Denotando por $(t, x)$ um ponto genérico de $\mathbb{R} \times \mathbb{R}_{+}^{*}$ e usando (iv) e (v) temos que

$$
\begin{aligned}
& \frac{\partial h}{\partial x}(t, x) \geq 0, \quad \text { para todo }(t, x) \in I \times \mathbb{R}_{+}^{*}, \\
& h(t, x) \geq 0, \quad \text { para todo }(t, x) \in I \times \mathbb{R}_{+}^{*} .
\end{aligned}
$$

Para (I) tomemos $K^{\prime} \subset \subset I \times \mathbb{R}_{+}^{*}$ e sejam $L \subset \subset I$ e $K \subset \subset \mathbb{R}_{+}^{*}$ tais que $K^{\prime} \subset L \times K$.

Como $l \in \mathcal{G}_{*}\left(\mathbb{R}_{+}^{*} ; \mathbb{R}_{+}^{*}\right)$ existem $K_{1} \subset \subset \mathbb{R}_{+}^{*}$ e $\left.\left.\eta \in\right] 0,1\right]$ tais que $\hat{l}(] 0, \eta[\times K) \subset K_{1}$.

Seja $a>0$ tal que $K \subset[a, \infty[$. Então, usando (iii), (iv) e (4) temos que

$$
0<a \leq x \leq h(t, \widehat{l}(\varepsilon, x))+x \leq h(t, g(x))+x
$$

para todo $(\varepsilon, t, x) \in] 0, \eta[\times L \times K$, e assim se $\bar{c}=\max \{h(t, g(x))+x:(t, x) \in L \times K\}$, temos

$$
\widehat{f}(] 0, \eta\left[\times K^{\prime}\right) \subset L \times[a, \bar{c}] \subset \subset \mathbb{R} \times \mathbb{R}_{+}^{*},
$$

e $\operatorname{assim}(\mathrm{I})$ é verdadeira.

De (ii) e (3) temos que

$$
\left.\left.J \widehat{f}(\varepsilon, t, x)=\frac{\partial h}{\partial x}(t, \widehat{l}(\varepsilon, x)) \widehat{l}^{\prime}(\varepsilon, x)+1 \geq 1, \text { para todo }(\varepsilon, t, x) \in\right] 0,1\right] \times I \times \mathbb{R}_{+}^{*},
$$

o que prova (II).

Provaremos a seguir, usando 1.2.15, as asserções (III) e (IV) .

Fixemos $\varepsilon \in] 0,1]$.

Provaremos que $\hat{f}(\varepsilon,$.$) é uma aplicação bijetora.$

Sejam $(s, x)$ e $(t, y)$ pertencentes a $I \times \mathbb{R}_{+}^{*}$. Se $s \neq t$ é claro que $\hat{f}(\varepsilon, s, x) \neq \hat{f}(\varepsilon, t, y)$. Suponhamos $s=t$, e portanto $x \neq y$.

Seja $\omega$ a função definida em $\mathbb{R}_{+}^{*}$ por 


$$
\omega(z)=h(s, \hat{l}(\varepsilon, z))+z .
$$

Então, usando (ii) e (3), temos

$$
\omega^{\prime}(z)=\frac{\partial h}{\partial x}(s, \widehat{l}(\varepsilon, z)) \widehat{l}^{\prime}(\varepsilon, z)+1>0, \text { para todo } z>0,
$$

e portanto $\omega$ é uma função estritamente crescente, e como $x \neq y$ temos que

$$
\widehat{f}(\varepsilon, s, x)=(s, \omega(x)) \neq(s, \omega(y))=\widehat{f}(\varepsilon, s, y) .
$$

Portanto $\hat{f}(\varepsilon,$.$) é uma aplicação injetora.$

Sejam $(s, y) \in I \times \mathbb{R}_{+}^{*}$ e $\omega$ como em (5). Então, por (iv), (v), (1) e (2) temos

$$
\lim _{z \downarrow 0} \omega(z)=0 \quad \text { e } \quad \lim _{z \uparrow \infty} \omega(z)=\infty
$$

e como $\omega$ é uma função estritamente crescente concluímos que $\omega\left(\mathbb{R}_{+}^{*}\right)=\mathbb{R}_{+}^{*}$, e assim existe $\bar{x}>0$ tal que $\omega(\bar{x})=y$.

Portanto

$$
\widehat{f}(\varepsilon, s, \bar{x})=(s, h(s, \widehat{l}(\varepsilon, \bar{x}))+\bar{x})=(s, \omega(\bar{x}))=(s, y),
$$

e $\operatorname{assim} \hat{f}(\varepsilon,$.$) é uma aplicação sobrejetora.$

Portanto estão satisfeitas as asserções 1.2.15.i e 1.2.15.ii.

Fixemos $\varepsilon \in] 0,1]$ e seja $g_{\varepsilon}$ a aplicação inversa de $\widehat{f}(\varepsilon,$.$) .$

Para verificar 1.2.15.iii tomemos $K^{\prime} \subset \subset I \times \mathbb{R}_{+}^{*}$ e sejam $L \subset \subset I$ e $K \subset \subset \mathbb{R}_{+}^{*}$ tais que $K^{\prime} \subset L \times K$.

Usando (vi) e o fato que $K \subset \subset \mathbb{R}_{+}^{*}$ podemos tomar $a_{1}>0, b_{1}>0$ e $c_{1}>0$ tais que $K \subset\left[a_{1}, b_{1}\right]$ e $h(t, x) \leq h\left(c_{1}, x\right)$ para todo $(t, x) \in L \times \mathbb{R}_{+}^{*}$. Então, por (iii), (iv) e (4), temos

$$
x \leq h(t, \widehat{l}(\varepsilon, x))+x \leq h(t, g(x))+x \leq h\left(c_{1}, g(x)\right)+x,
$$

para todo $(t, x) \in L \times \mathbb{R}_{+}^{*}$.

Seja $\varphi$ a função definida em $\mathbb{R}_{+}^{*}$ por $\varphi(x)=h\left(c_{1}, g(x)\right)+x$. Então, por (iii), (iv), (v) e (2) temos que $\varphi$ é uma função estritamente crescente,

$$
\lim _{x \downarrow 0} \varphi(x)=0 \quad \text { e } \quad \lim _{x \uparrow \infty} \varphi(x)=\infty
$$


e $\operatorname{assim} \varphi\left(\mathbb{R}_{+}^{*}\right)=\mathbb{R}_{+}^{*}$.

Portanto, por (6), temos que

$$
\begin{aligned}
\left\{g_{\varepsilon}(s, y):(s, y) \in K^{\prime}\right\} & \subset\left\{(s, x) \in L \times \mathbb{R}_{+}^{*}: \hat{f}(\varepsilon, s, x) \in K^{\prime}\right\} \\
& \subset\left\{(s, x) \in L \times \mathbb{R}_{+}^{*}: a_{1} \leq h(s, \widehat{l}(\varepsilon, x))+x \leq b_{1}\right\} \\
& \left.\subset L \times(]-\infty, b_{1}\right] \cap \varphi^{-1}\left(\left[a_{1}, \infty[)\right) .\right.
\end{aligned}
$$

Como $\varphi\left(\mathbb{R}_{+}^{*}\right)=\mathbb{R}_{+}^{*}$ e $\varphi$ é uma função estritamente crescente, existe $d_{1}>0$ tal que $\varphi^{-1}\left(\left[a_{1}, \infty[)=\left[d_{1}, \infty\left[\right.\right.\right.\right.$ e $d_{1} \leq \varphi\left(d_{1}\right)=a_{1}<b_{1}$.

Portanto

$$
\left\{g_{\varepsilon}(s, y):(s, y) \in K^{\prime}\right\} \subset L \times\left[d_{1}, b_{1}\right] \subset \subset I \times \mathbb{R}_{+}^{*} .
$$

Como $d_{1}$ e $b_{1}$ não dependem de $\left.\left.\varepsilon \in\right] 0,1\right]$ temos que

$$
\left.\left.\left\{g_{\varepsilon}(s, y):(s, y) \in K^{\prime} \text { e } \varepsilon \in\right] 0,1\right]\right\} \subset L \times\left[d_{1}, b_{1}\right] \subset \subset I \times \mathbb{R}_{+}^{*},
$$

o que prova 1.2.15.iii.

De (II) e 1.1.32 temos que são verdadeiras 1.2.15.iv e 1.2.15.a.

Portanto (III) e (IV) são verdadeiras(1.2.15). //

Para completar o resultado acima apresentaremos, a seguir, algumas funções que satisfazem as hipóteses do mesmo.

1.2.27 Exemplo. Sejam $p \in \mathbb{N}^{*}$, h uma função definida em $\left.\left.\left.] 0,1\right] \operatorname{com} h(] 0,1\right]\right) \subset\left[\frac{1}{2}, 1\right]$ $e \varphi \in \mathcal{C}^{\infty}\left(\mathbb{R}_{+}^{*} ; \mathbb{R}_{+}^{*}\right) \operatorname{com} \varphi^{\prime}\left(\mathbb{R}_{+}^{*}\right) \subset \mathbb{R}_{+}^{*} e \lim _{x \downarrow 0} \varphi(x)=0$. Se $\hat{l}_{1}, \hat{l}_{2}$ e $\hat{l}_{3}$ são as funções moderadas definidas em $] 0,1] \times \mathbb{R}_{+}^{*}$ por

$$
\hat{l}_{1}(\varepsilon, x)=h(\varepsilon) \varphi(x) \quad, \quad \hat{l}_{2}(\varepsilon, x)=\varphi\left(\left(h(\varepsilon) x^{p}\right)\right) \quad \text { e } \hat{l}_{3}(\varepsilon, x)=x^{h(\varepsilon)}
$$

e $l_{j}$ é a classe de $\hat{l}_{j}$ em $\mathcal{G}\left(\mathbb{R}_{+}^{*} ; \mathbb{R}\right)$, para todo $1 \leq j \leq 3$, então $l_{j} \in \mathcal{G}_{*}\left(\mathbb{R}_{+}^{*} ; \mathbb{R}_{+}^{*}\right)$ e $\hat{l}_{j}$ satisfaz de 1.2.26.i até 1.2.26.iii, para todo $1 \leq j \leq 3$. As funções definidas em $\mathbb{R} \times \mathbb{R}_{+}^{*}$ por

$$
h_{1}(t, x)=\ln \left(x t^{2}+1\right) \quad \text { e } \quad h_{2}(t, x)=t^{2} \operatorname{arctg} x
$$


pertencem a $\mathcal{C}^{\infty}\left(\mathbb{R} \times \mathbb{R}_{+}^{*} ; \mathbb{R}\right)$ e satisfazem de 1.2.26.iv até 1.2.26.vi.

(Convém observar que $\widehat{l}_{3}(\varepsilon, x) \leq g(x)$, sendo $g(x)=\sqrt{x}$ se $0<x \leq 1$ e $g(x)=x$ se $x>1$. As funções $\Phi_{1}(x)=x^{r}$, sendo $r \in \mathbb{R}, \Phi_{2}(x)=\ln (1+x)$ e $\Phi_{3}(x)=\int_{0}^{x} \exp \left(-t^{2}\right) d t$ podem, por exemplo, ser usadas para $\varphi$.)

Analisando a prova de 1.2.26 obtemos o seguinte resultado:

1.2.28 Proposição. Sejam $I$ um intervalo aberto de $\mathbb{R}$ com $0 \in I, 1 \leq k \leq n, l_{k} \in$ $\mathcal{G}_{*}\left(\mathbb{R}_{+}^{*} ; \mathbb{R}_{+}^{*}\right), \hat{l}_{k}$ um representante de $l_{k}$ e $h_{k} \in \mathcal{C}^{\infty}\left(I \times \mathbb{R}_{+}^{*} ; \mathbb{R}\right)$ tais que

(i) $\hat{l}_{k}$ satisfaz de 1.2.26.i até 1.2.26.iii (substituindo $l$ por $l_{k}$ );

(ii) $h_{k}$ satisfaz de 1.2.26.iv até 1.2.26.vi (substituindo $h$ por $h_{k}$ ).

Se $\hat{f}$ é a aplicação moderada definida em $] 0,1 \times I \times \mathbb{R}_{+}^{* n}$ por

$$
\widehat{f}\left(\varepsilon, t, x_{1}, \ldots, x_{n}\right)=\left(t, h_{1}\left(t, \hat{l}_{1}\left(\varepsilon, x_{1}\right)\right)+x_{1}, \ldots, h_{n}\left(t, \widehat{l}_{n}\left(\varepsilon, x_{n}\right)\right)+x_{n}\right)
$$

e f é a classe de $\hat{f}$ em $\mathcal{G}\left(I \times \mathbb{R}_{+}^{* n} ; \mathbb{R}^{n+1}\right)$, entâo

(I) $f \in \mathcal{G}_{*}\left(I \times \mathbb{R}_{+}^{* n} ; I \times \mathbb{R}_{+}^{* n}\right)$;

(II) $\left.\left.\inf \left\{J \widehat{f}\left(\varepsilon, t, x_{1}, \ldots, x_{n}\right):\left(\varepsilon, t, x_{1}, \ldots, x_{n}\right) \in\right] 0,1\right] \times I \times \mathbb{R}_{+}^{* n}\right\} \geq 1$;

(III) $f$ é uma aplicação inversível;

(IV) $\widehat{f}(\varepsilon,$.$) é uma aplicação inversível para todo \varepsilon \in] 0,1]$ e a aplicação moderada $\widehat{\Gamma}$ definida em $] 0,1] \times I \times \mathbb{R}_{+}^{* n}$ por $\Gamma(\varepsilon,)=.(\widehat{f}(\varepsilon, .))^{-1}$ para todo $\left.\left.\varepsilon \in\right] 0,1\right]$, é um representante de $f^{-1} \cdot / /$

A seguir apresentamos um resultado para o caso em que $\Omega=] 0,1[$.

1.2.29 Proposição. Sejam I um intervalo aberto de $\mathbb{R}$ com $0 \in I, l \in \mathcal{G}_{*}(] 0,1[;] 0,1[)$, $\hat{l}$ um representante de $l$ satisfazendo: 
(i) $\hat{l}(] 0,1] \times] 0,1[) \subset] 0,1[$;

(ii) $\widehat{l}^{\prime}(\varepsilon, x) \geq 0$, para todo $\left.\left.\left.(\varepsilon, x) \in\right] 0,1\right] \times\right] 0,1[$;

(iii) existem $\varphi_{1}$ e $\varphi_{2}$ pertencentes a $\mathcal{C}([0,1] ; \mathbb{R})$ tais que

$$
\begin{gathered}
\varphi_{1}(0)=\varphi_{2}(0)=0 \quad ; \quad \varphi_{1}(1)=\varphi_{2}(1)=1 ; \\
\left.\left.\left.0 \leq \varphi_{1}(x) \leq \widehat{l}(\varepsilon, x) \leq \varphi_{2}(x) \leq 1, \quad \text { para todo }(\varepsilon, x) \cong\right] 0,1\right] \times\right] 0,1[,
\end{gathered}
$$

e seja $h \in \mathcal{C}^{\infty}(] 0,1[; \mathbb{R}) \cap \mathcal{C}([0,1] ; \mathbb{R})$ tal que

(iv) $h(0)=0$ e $h^{\prime}(x) \geq 0$, para todo $\left.x \in\right] 0,1[$.

Se $W=\left\{(t, y) \in I \times \mathbb{R}: 0<y<1+t^{2} h(1)\right\}, \hat{f}$ é a aplica̧̧ão moderada definida em ]0,1] $\times I \times] 0,1[$ por

$$
\widehat{f}(\varepsilon, t, x)=\left(t, t^{2} h(\widehat{l}(\varepsilon, x))+x\right)
$$

e f é a classe de $\hat{f}$ em $\mathcal{G}(I \times] 0,1\left[; \mathbb{R}^{2}\right)$, então

(I) $f \in \mathcal{G}_{*}(I \times] 0,1[; W)$;

(II) $\inf \{J \widehat{f}(\varepsilon, t, x):(\varepsilon, t, x) \in] 0,1] \times I \times] 0,1[\} \geq 1$;

(III) $f$ é uma aplicação inversivel;

(IV) $\widehat{f}(\varepsilon$, .) é uma aplicação inversível para todo $\varepsilon \in] 0,1]$ e a aplicação moderada $\widehat{\Gamma}$ definida em $] 0,1] \times W$ por $\hat{\Gamma}(\varepsilon,)=.(\widehat{f}(\varepsilon, .))^{-1}$ para todo $\left.\left.\varepsilon \in\right] 0,1\right]$, é um representante de $f^{-1}$.

(Convém observar que a hipótese (i) é utilizada somente para definir a aplicação $\widehat{f}$.)

Demonstraçấo. Notemos, em primeiro lugar, que, por (iii), temos

$$
\left.\left.\lim _{x \downarrow 0} \hat{l}(\varepsilon, x)=0 \quad \text { e } \lim _{x \uparrow 1} \hat{l}(\varepsilon, x)=1 \text {, para todo } \varepsilon \in\right] 0,1\right] .
$$

Provaremos a seguir (I).

Seja $\left.K^{\prime} \subset \subset I \times\right] 0,1[$ e tomemos $L \subset \subset I$ e $K \subset \subset] 0,1\left[\right.$ tais que $K^{\prime} \subset L \times K$. 
Como $l \in \mathcal{G}_{*}(] 0,1[;] 0,1[)$ existem $\left.K_{1} \subset \subset\right] 0,1[$ e $\left.\eta \in] 0,1\right]$ tais que $\hat{l}(] 0, \eta[\times K) \subset$ $K_{1}$. Então

$$
\{\hat{f}(\varepsilon, t, x):(\varepsilon, t, x) \in] 0, \eta\left[\times K^{\prime}\right\} \subset\left\{\left(t, t^{2} h(z)+x\right):(t, x, z) \in L \times K \times K_{1}\right\} .
$$

Seja $\psi$ a função definida em $I \times] 0,1[\times] 0,1[$ por

$$
\psi(t, x, z)=\left(t, t^{2} h(z)+x\right) .
$$

Então $\psi \in \mathcal{C}(I \times] 0,1[\times] 0,1[; \mathbb{R})$ e como, por (iv),

$$
0<t^{2} h(z)+x \leq t^{2} h(1)+x<t^{2} h(1)+1
$$

temos que $\psi(I \times] 0,1[\times] 0,1[) \subset W$ e portanto, de $(2)$, temos que

$$
\{\widehat{f}(\varepsilon, t, x):(\varepsilon, t, x) \in] 0, \eta\left[\times K^{\prime}\right\} \subset \psi\left(L \times K \times K_{1}\right) \subset \subset W
$$

e $\operatorname{assim}(\mathrm{I})$ é verdadeira.

De (ii) e (iv) concluímos que

$$
\left.\left.\left.J \widehat{f}(\varepsilon, t, x)=t^{2} h^{\prime}(\widehat{l}(\varepsilon, x)) \widehat{l}^{\prime}(\varepsilon, x)+1 \geq 1, \text { para todo }(\varepsilon, t, x) \in\right] 0,1\right] \times I \times\right] 0,1[
$$

o que prova (II).

Provaremos a seguir, usando 1.2.15, as asserções (III) e (IV) .

Fixemos $\varepsilon \in] 0,1]$.

Provaremos que $\hat{f}(\varepsilon,$.$) é uma aplicação bijetora. Para isto, dado t \in I$, seja $l_{t}$ a função definida em ]0,1[ por

$$
l_{t}(x)=x+t^{2} h(\widehat{l}(\varepsilon, x)) .
$$

Então, por (ii), (iv) e (1), temos que $l_{t}$ é uma função estritamente crescente, $\lim _{x \downarrow 0} l_{t}(x)=0$ e $\lim _{x \uparrow 1} l_{t}(x)=1+t^{2} h(1)$, e assim $\left.l_{t}(] 0,1[)=\right] 0,1+t^{2} h(1)[$.

Sejam $(s, x)$ e $(t, y)$ pertencentes a $I \times] 0,1[$. Se $s \neq t$ é claro que $\hat{f}(\varepsilon, s, x) \neq$ $\widehat{f}(\varepsilon, t, y)$. Suponhamos $s=t$, e portanto $x \neq y$.

Como $x \neq y$ e $l_{t}$ é uma função estritamente crescente temos que

$$
\widehat{f}(\varepsilon, t, x)=\left(t, t^{2} h(\widehat{l}(\varepsilon, x))+x\right)=\left(t, l_{t}(x)\right) \neq\left(t, l_{t}(y)\right)=\widehat{f}(\varepsilon, t, y) .
$$


Portanto $\hat{f}(\varepsilon,$.$) é uma aplicação injetora.$

Seja $(t, y) \in W$. Então $0<y<1+t^{2} h(1)$, e portanto $y \in l_{t}(] 0,1[)$. Como $l_{t}$ é contínua em ]0,1[, existe $x \in] 0,1\left[\right.$ tal que $l_{t}(x)=y$, e assim

$$
\widehat{f}(\varepsilon, t, x)=\left(t, l_{t}(x)\right)=(t, y)
$$

ou seja $\hat{f}(\varepsilon,$.$) é uma aplicação sobrejetora.$

Portanto estão satisfeitas as asserções 1.2.15.i e 1.2.15.ii.

Fixemos $\varepsilon \in] 0,1]$ e seja $g_{\varepsilon}$ a aplicação inversa de $\widehat{f}(\varepsilon,$.$) .$

Para verificar 1.2.15.iii tomemos $K^{\prime} \subset \subset W$.

Como $K^{\prime} \subset \subset W$, existe uma seqüência finita de compactos $\left(L_{j}\right)_{1 \leq j \leq k}$, sendo $L_{j}=$ $\left[a_{j}, b_{j}\right] \times\left[c_{j}, d_{j}\right]$, tal que $K^{\prime} \subset \cup_{j=1}^{k} L_{j} \subset W$, e portanto temos que

$$
\begin{aligned}
\left\{g_{\varepsilon}(s, y):(s, y) \in K^{\prime}\right\} & \subset \cup_{j=1}^{k}\left\{g_{\varepsilon}(s, y):(s, y) \in L_{j}\right\} \\
& \subset \cup_{j=1}^{k}\left\{(s, x) \in\left[a_{j}, b_{j}\right] \times\right] 0,1\left[: \widehat{f}(\varepsilon, s, x) \in L_{j}\right\} \\
& \subset \cup_{j=1}^{k}\{(s, x) \in I \times] 0,1\left[: s \in\left[a_{j}, b_{j}\right] \mathrm{e} c_{j} \leq x+s^{2} h(\hat{l}(\varepsilon, x)) \leq d_{j}\right\} .
\end{aligned}
$$

Fixemos $j \in\{1, \ldots, k\}$ e sejam $\varphi_{1}$ e $\varphi_{2}$ como em (iii) e $\omega_{1}$ e $\omega_{2}$ as funções definidas em $I \times] 0,1[$ por

$$
\omega_{1}(t, x)=\left(t, t^{2} h\left(\varphi_{1}(x)\right)+x\right) \quad \text { e } \quad \omega_{2}(t, x)=\left(t, t^{2} h\left(\varphi_{2}(x)\right)+x\right) .
$$

Então, usando (iii) e (iv), temos que

$$
\begin{gathered}
\left.0<t^{2} h\left(\varphi_{i}(x)\right)+x \leq t^{2} h(1)+x<t^{2} h(1)+1, \text { para todo } x \in\right] 0,1[\text { e } 1 \leq i \leq 2 ; \\
\lim _{x \downarrow 0} \omega_{i}(t, x)=(t, 0) \quad \text { e } \quad \lim _{x \uparrow 1} \omega_{i}(t, x)=\left(t, 1+t^{2} h(1)\right), \text { para todo } t \in I \text { e } 1 \leq i \leq 2,
\end{gathered}
$$

e portanto $\omega_{1}(I \times] 0,1[)=\omega_{2}(I \times] 0,1[)=W$.

Seja $A_{j}=\{(s, x) \in I \times] 0,1\left[: s \in\left[a_{j}, b_{j}\right]\right.$ e $\left.c_{j} \leq x+s^{2} h(\widehat{l}(\varepsilon, x)) \leq d_{j}\right\}$.

Como temos, por (iii) e (iv),

$$
\left.x+t^{2} h\left(\varphi_{1}(x)\right) \leq x+t^{2} h(\widehat{l}(\varepsilon, x)) \leq x+t^{2} h\left(\varphi_{2}(x)\right), \text { para todo }(t, x) \in I \times\right] 0,1[,
$$


concluímos que

$$
\left.A_{j} \subset \omega_{2}^{-1}\left(\left[a_{j}, b_{j}\right] \times\left[c_{j}, \infty[) \cap \omega_{1}^{-1}\left(\left[a_{j}, b_{j}\right] \times\right]-\infty, d_{j}\right]\right) \subset\left[a_{j}, b_{j}\right] \times\right] 0,1[.
$$

Sejam

$$
\begin{gathered}
\left.\left.M_{j}=\sup \{x \in] 0,1\left[: \text { existe } s \in \mathbb{R} \operatorname{com}(s, x) \in \omega_{1}^{-1}\left(\left[a_{j}, b_{j}\right] \times\right]-\infty, d_{j}\right]\right)\right\} ; \\
m_{j}=\inf \{x \in] 0,1\left[: \text { existe } s \in \mathbb { R } \operatorname { c o m } ( s , x ) \in \omega _ { 2 } ^ { - 1 } \left(\left[a_{j}, b_{j}\right] \times\left[c_{j}, \infty[)\right\} .\right.\right.
\end{gathered}
$$

Então $M_{j} \leq 1$ e $m_{j} \geq 0$. Provaremos que $M_{j}<1$ e que $m_{j}>0$.

Suponhamos, por absurdo, $M_{j}=1$. Então existe uma seqüência $\left(s_{n}, x_{n}\right)_{n \in N}$ com $\left.\left.\left(s_{n}, x_{n}\right) \in \omega_{1}^{-1}\left(\left[a_{j}, b_{j}\right] \times\right]-\infty, d_{j}\right]\right)$ e tal que $\lim _{n \uparrow \infty} x_{n}=1$. Como $s_{n} \in\left[a_{j}, b_{j}\right]$ para todo $n \in \mathbb{N}$, e $\left[a_{j}, b_{j}\right] \subset \subset \mathbb{R}$, existe uma subseqüência $\left(s_{n_{k}}\right)_{k \in N}$ que converge para algum $s \in\left[a_{j}, b_{j}\right]$. Então

$$
x_{n_{k}}+s_{n_{k}}^{2} h\left(\varphi_{1}\left(x_{n_{k}}\right)\right) \leq d_{j} \quad \text { e } \quad \lim _{k \uparrow \infty}\left(x_{n_{k}}+s_{n_{k}}^{2} h\left(\varphi_{1}\left(x_{n_{k}}\right)\right)\right)=1+s^{2} h(1)
$$

e portanto $d_{j} \geq 1+s^{2} h(1)$, o que é um absurdo, pois $\left(s, d_{j}\right) \in W$. De modo análogo prova-se que $m_{j}>0$.

Portanto

$\omega_{2}^{-1}\left(\left[a_{j}, b_{j}\right] \times\left[c_{j}, \infty[) \cap \omega_{1}^{-1}\left(\left[a_{j}, b_{j}\right] \times\right]-\infty, d_{j}\right]\right) \subset\left(\left[a_{j}, b_{j}\right] \times\left[m_{j}, 1[) \cap\left(\left[a_{j}, b_{j}\right] \times\right] 0, M_{j}\right]\right)$, e assim (convencionando que $\left[m_{j}, M_{j}\right]=\emptyset$ se $m_{j}>M_{j}$ ) concluímos que $A_{j} \subset\left[a_{j}, b_{j}\right] \times$ $\left[m_{j}, M_{j}\right]$.

Portanto

$$
\left.\left\{g_{\varepsilon}(s, x):(s, y) \in K^{\prime}\right\} \subset \cup_{j=1}^{k} A_{j} \subset \cup_{j=1}^{k}\left(\left[a_{j}, b_{j}\right] \times\left[m_{j}, M_{j}\right]\right) \subset \subset I \times\right] 0,1[,
$$

o que prova 1.2.15.iii.

De (II) e 1.1.32 temos que são verdadeiras 1.2.15.iv e 1.2.15.a, e assim concluímos, por 1.2 .15$, (III) e (IV). //

Completamos o resultado acima apresentando funções que satisfazem as hipóteses do mesmo. 
1.2.30 Exemplo. Sejam $p \in N^{*}$ e $\mu$ e $\lambda$ funções definidas em ]0,1] e com valores em $\mathbb{R}$ tais que $\left.\left.\mu(] 0,1]) \cup \lambda(] 0,1]) \subset] 0,1], \frac{1}{\lambda} \in \mathcal{E}_{M}(\mathbb{R}) e \inf \{\mu(\varepsilon): \varepsilon \in] 0,1\right]\right\}>0$. Se $\hat{l}_{1}$ e $\hat{l}_{2}$ são as funções moderadas definidas em ]0,1]×]0,1[ por

$$
\hat{l}_{1}(\varepsilon, x)=x^{\mu(\varepsilon)} \quad \text { e } \quad \hat{l}_{2}(\varepsilon, x)=1+\lambda(\varepsilon)-\sqrt{(\lambda(\varepsilon))^{2}+(\lambda(\varepsilon)+1)^{2}-(\lambda(\varepsilon)+x)^{2}}
$$

e $l_{j}$ a classe de $\hat{l}_{j}$ em $\mathcal{G}(] 0,1[; \mathbb{R})$, para todo $1 \leq j \leq 2$, então $l_{j} \in \mathcal{G}_{*}(] 0,1[;] 0,1[) e$ $\hat{l}_{j}$ satisfaz de 1.2.29.i até 1.2.29.iii, para todo $1 \leq j \leq 2$. As funçôes definidas em $[0,1]$ por

$$
h_{1}(x)=\operatorname{sen}\left(x^{p}\right), \quad h_{2}(x)=\ln \left(1+x^{p}\right) \quad \text { e } \quad h_{3}(x)=x^{p}
$$

pertencem a $\mathcal{C}^{\infty}(] 0,1[; \mathbb{R}) \times \mathcal{C}([0,1] ; \mathbb{R})$ e satisfazem 1.2.29.iv.

(Convém observar que a função $\widehat{l}_{2}-\hat{1}$ foi estudada em 1.2.7.)

Analisando a prova de 1.2 .29 obtemos o seguinte resultado.

1.2.31 Proposição. Sejam I um intervalo aberto de $\mathbb{R}$ com $0 \in I, 1 \leq k \leq n, l_{k} \in$ $\mathcal{G}_{*}(] 0,1[;] 0,1[), \hat{l}_{k}$ um representante de $l_{k}$ e $h_{k} \in \mathcal{C}^{\infty}(] 0,1[; \mathbb{R}) \cap \mathcal{C}([0,1] ; \mathbb{R})$ tais que

(i) $\hat{l}_{k}$ satisfaz de 1.2.29.i até 1.2.29.iii (substituindo $\hat{l}$ por $\hat{l}_{k}$ );

(ii) $h_{k}$ satisfaz 1.2.29.iv (substituindo $h$ por $h_{k}$ ).

Se $W=\left\{\left(t, y_{1}, \ldots, y_{n}\right) \in I \times \mathbb{R}^{n}: 0<y_{j}<1+t^{2} h_{j}(1)\right.$, para todo $\left.1 \leq j \leq n\right\}$, $\hat{f}$ é $a$ aplicação moderada definida em $] 0,1] \times I \times] 0,1\left[{ }^{n}\right.$ por

$$
\widehat{f}\left(\varepsilon, t, x_{1}, \ldots, x_{n}\right)=\left(t, t^{2} h_{1}\left(\widehat{l}_{1}\left(\varepsilon, x_{1}\right)\right)+x_{1}, \ldots, t^{2} h_{n}\left(\widehat{l}_{n}\left(\varepsilon, x_{n}\right)\right)+x_{n}\right)
$$

e fé a classe de $\widehat{f}$ em $\mathcal{G}(I \times] 0,1\left[{ }^{n} ; \mathbb{R}^{n+1}\right)$, então

(I) $\left.f \in \mathcal{G}_{*}(I \times] 0,1{ }^{n} ; W\right)$;

(II) $\left.\left.\left.\left.\inf \left\{J \hat{f}\left(\varepsilon, t, x_{1}, \ldots, x_{n}\right):\left(\varepsilon, t, x_{1}, \ldots, x_{n}\right) \in\right] 0,1\right] \times I \times\right] 0,1\right]^{n}\right\} \geq 1$;

(III) $f$ é uma aplicação inversível; 
(IV) $\widehat{f}(\varepsilon,$.$) é uma aplicação inversível para todo \varepsilon \in] 0,1]$ e a aplicação moderada $\widehat{\Gamma}$ definida em $] 0,1] \times W$ por $\hat{\Gamma}(\varepsilon,)=.(\widehat{f}(\varepsilon, .))^{-1}$ para todo $\left.\left.\varepsilon \in\right] 0,1\right]$, é um representante de $f^{-1}$. // 


\section{Capítulo 2}

\section{Equação de Hamilton-Jacobi :}

\section{Existência de soluções}

Para facilitar a escrita do problema que iremos estudar, e que será chamado problema HJ, convencionaremos que

(1) $\widehat{\pi}$ denotará a função moderada definida em $] 0,1] \times \mathbb{R} \times \mathbb{R}^{n}$ por $\widehat{\pi}\left(\varepsilon, t, x_{1}, \ldots, x_{n}\right)=t$;

(2) $\pi$ denotará, por abuso de notação, não somente a classe de $\hat{\pi}$ em $\mathcal{G}\left(\mathbb{R}^{n+1} ; \mathbb{R}\right)$ como também a classe de $\left.\widehat{\pi}\right|_{[0,1] \times W}$ em $\mathcal{G}(W ; \mathbb{R})$, sendo $W$ um aberto qualquer de $\mathbb{R}^{n+1}$

(3) $\widehat{\pi}_{j}$, para todo $1 \leq j \leq n$, denotará a função moderada definida em $\left.] 0,1\right] \times \mathbb{R} \times \mathbb{R}^{n}$ por $\hat{\pi}_{j}\left(\varepsilon, t, x_{1}, \ldots, x_{n}\right)=x_{j}$,

(4) $\pi_{j}$ denotará, por abuso de notação, não somente a classe de $\hat{\pi}_{j}$ em $\mathcal{G}\left(\mathbb{R}^{n+1} ; \mathbb{R}\right)$ como também a classe de $\left.\widehat{\pi}_{j}\right|_{j 0,1] \times W}$ em $\mathcal{G}(W ; \mathbb{R})$, para todo $1 \leq j \leq n$, sendo $W$ um aberto qualquer de $\mathbb{R}^{n+1}$.

Convencionaremos também que, se $W$ é um aberto de $\mathbb{R}^{n+1}, u \in \mathcal{G}(W ; \mathbb{R}),(t, x)=$ $\left(t, x_{1}, \ldots, x_{n}\right)$ denota um ponto genérico de $\mathbb{R} \times \mathbb{R}^{n}, \quad \alpha=\left(\alpha_{0}, \alpha_{1}, \ldots, \alpha_{n}\right) \in \mathbb{N}^{n+1}$ com $|\alpha|=1$ e $1 \leq j \leq n$, então 


$$
\begin{aligned}
& \frac{\partial u}{\partial t} \text { denotará a função } \partial^{\alpha} u \text { se } \alpha_{0}=1 ; \\
& \frac{\partial u}{\partial x_{j}} \text { denotará a função } \partial^{\alpha} u \text { se } \alpha_{j}=1 .
\end{aligned}
$$

O problema que estudamos e que, sob certas hipóteses, obtivemos em 2.1.2 uma solução é o seguinte:

Problema $\mathbf{H J}$ : Dados quaisquer I intervalo aberto de $\mathbb{R}$ com $0 \in I, \Omega$ e $\Omega^{\prime}$ abertos de $\mathbb{R}^{n}, H \in \mathcal{G}\left(I \times \Omega \times \Omega^{\prime} ; \mathbb{R}\right)$ e $f \in \mathcal{G}(\Omega ; \mathbb{R})$, determinar um aberto $W$ de $I \times \Omega$ com $V=\left\{z \in \mathbb{R}^{n}:(0, z) \in W\right\} \neq \emptyset$ e uma função $u \in \mathcal{G}(W ; \mathbb{R})$ satisfazendo:

(i) $\left(\frac{\partial u}{\partial x_{1}}, \ldots, \frac{\partial u}{\partial x_{n}}\right) \in \mathcal{G}_{*}\left(W ; \Omega^{\prime}\right)$;

(ii) $\frac{\partial u}{\partial t}+H \circ\left(\pi, \pi_{1}, \ldots, \pi_{n}, \frac{\partial u}{\partial x_{1}}, \ldots, \frac{\partial u}{\partial x_{n}}\right)=0 \quad$ em $\mathcal{G}(W ; \mathbb{R})$;

(iii) $\left.u\right|_{\{0\} \times V}:=\left.f\right|_{V} \quad$ em $\mathcal{G}(V ; \mathbb{R})$.

Dizemos que $u \in \mathcal{G}(W ; \mathbb{R})$ é uma solução para o problema $\mathbf{H J}$ em $\mathcal{G}(W ; \mathbb{R})$ se, e somente se, $u$ satisfaz (i), (ii) e (iii).

No caso clássico, isto é, quando $H$ e $f$ são funções de classe $\mathcal{C}^{\infty}$, a equação (ii) é chamada equação de Hamilton-Jacobi e é escrita da seguinte forma:

$$
\frac{\partial u}{\partial t}+H\left(t, x_{1}, \ldots, x_{n}, \frac{\partial u}{\partial x_{1}}, \ldots, \frac{\partial u}{\partial x_{n}}\right)=0
$$

A equação de Hamilton-Jacobi é uma equação diferencial parcial de primeira ordem, não linear, onde não aparece a função incógnita $u$. Esta equação aparece freqüentemente na Mecânica Analítica, como por exemplo o caso do oscilador harmônico, cuja equação de Hamilton-Jacobi é:

$$
\frac{1}{2 m}\left(\frac{\partial u}{\partial x}\right)^{2}+\frac{k x^{2}}{2}+\frac{\partial u}{\partial t}=0
$$

sendo $k$ uma constante e $x$ a abscissa da partícula no instante $t$. 
O problema $\mathbf{H J}$, no caso clássico, sempre tem uma solução. Um método utilizado para encontrar essa solução é o método das características([4]). Este método consiste em transformar o problema HJ que é um problema de equações diferenciais parciais em um problema de equações diferenciais ordinárias. Em 2.1.2 procuramos adaptar o método das características para o caso em que $H$ e $f$ são funções generalizadas.

\subsection{Um teorema de existência de soluções para a equação de Hamilton-Jacobi}

Com o objetivo de facilitar a leitura dos resultados que apresentaremos faremos mais algumas notações e uma definição.

Se $I$ é um intervalo aberto de $\mathbb{R}$ e $\Omega$ e $\Omega^{\prime}$ são abertos de $\mathbb{R}^{n}$, então convencionaremos, como no caso clássico, o seguinte:

(1) se $x=\left(x_{1}, \ldots, x_{n}\right)$ denota um ponto genérico de $\mathbb{R}^{n}, f \in \mathcal{G}(\Omega ; \mathbb{R}), \hat{f}$ é um representante de $f$ e $\alpha=\left(\alpha_{1}, \ldots, \alpha_{n}\right) \in \mathbb{I}^{n}$ com $|\alpha|=1$, então

$$
\begin{aligned}
& \frac{\partial f}{\partial x_{j}} \text { denotará a função } \partial^{\alpha} f \text { se } \alpha_{j}=1 \text { e } 1 \leq j \leq n ; \\
& \frac{\partial \widehat{f}}{\partial x_{j}} \text { denotará a função } \partial^{\alpha} \widehat{f} \text { se } \alpha_{j}=1 \text { e } 1 \leq j \leq n ; \\
& \nabla f \text { denotará a aplicação }\left(\frac{\partial f}{\partial x_{1}}, \ldots, \frac{\partial f}{\partial x_{n}}\right) ; \\
& \nabla \widehat{f} \text { denotará a aplicação }\left(\frac{\partial \widehat{f}}{\partial x_{1}}, \ldots, \frac{\partial \widehat{f}}{\partial x_{n}}\right) ;
\end{aligned}
$$

(2) se $(t, x)=\left(t, x_{1}, \ldots, x_{n}\right)$ denota um ponto genérico de $\mathbb{R} \times \mathbb{R}^{n}$ e $g=\left(g_{1}, \ldots, g_{k}\right) \in$ $\mathcal{G}\left(I \times \Omega ; \mathbb{R}^{k}\right)$, então

$$
\frac{\partial g}{\partial t} \text { denotará a aplicação }\left(\frac{\partial g_{1}}{\partial t}, \ldots, \frac{\partial g_{k}}{\partial t}\right)
$$


(3) se $(t, x, p)=\left(t, x_{1}, \ldots, x_{n}, p_{1}, \ldots, p_{n}\right)$ denota um ponto genérico de $\mathbb{R} \times \mathbb{R}^{n} \times \mathbb{R}^{n}$, $H \in \mathcal{G}\left(I \times \Omega \times \Omega^{\prime} ; \mathbb{R}\right), \widehat{H}$ é um representante de $H, \gamma=\left(\alpha_{0}, \alpha_{1}, \ldots, \alpha_{n}, \beta_{1}, \ldots, \beta_{n}\right) \in$ $\mathbb{N}^{2 n+1}$ com $|\gamma|=1$ e $1 \leq j \leq n$, então

$$
\begin{aligned}
& \frac{\partial H}{\partial t} \text { denotará a função } \partial^{\gamma} H \text { se } \alpha_{0}=1 ; \\
& \frac{\partial \widehat{H}}{\partial t} \text { denotará a função } \partial^{\gamma} \widehat{H} \text { se } \alpha_{0}=1 ; \\
& \frac{\partial H}{\partial x_{j}} \text { denotará a função } \partial^{\gamma} H \text { se } \alpha_{j}=1 ; \\
& \frac{\partial \widehat{H}}{\partial x_{j}} \text { denotará a função } \partial^{\gamma} \widehat{H} \text { se } \alpha_{j}=1 ; \\
& \frac{\partial H}{\partial p_{j}} \text { denotará a função } \partial^{\gamma} H \text { se } \beta_{j}=1 ; \\
& \frac{\partial \widehat{H}}{\partial p_{j}} \text { denotará a função } \partial^{\gamma} \widehat{H} \text { se } \beta_{j}=1 ; \\
& \frac{\partial H}{\partial x} \text { denotará a aplicação }\left(\frac{\partial H}{\partial x_{1}}, \ldots, \frac{\partial H}{\partial x_{n}}\right) ; \\
& \frac{\partial \widehat{H}}{\partial x} \text { denotará a aplicação }\left(\frac{\partial \widehat{H}}{\partial x_{1}}, \ldots, \frac{\partial \widehat{H}}{\partial x_{n}}\right) ; \\
& \frac{\partial H}{\partial p} \text { denotará a aplicação }\left(\frac{\partial H}{\partial p_{1}}, \ldots, \frac{\partial H}{\partial p_{n}}\right) ; \\
& \frac{\partial \widehat{H}}{\partial p} \text { denotará a aplicação }\left(\frac{\partial \widehat{H}}{\partial p_{1}}, \ldots, \frac{\partial \widehat{H}}{\partial p_{n}}\right) .
\end{aligned}
$$

Aqui também usaremos a notação dada em 1.1 .10 e 1.2.21.

2.1.1 Definição. Sejam I um intervalo aberto de $\mathbb{R}$ com $0 \in I, \Omega$ e $\Omega^{\prime}$ abertos de $\mathbb{R}^{n}$, $H \in \mathcal{G}\left(I \times \Omega \times \Omega^{\prime} ; \mathbb{R}\right)$ e $f \in \mathcal{G}(\Omega ; \mathbb{R})$. Se $J$ é um intervalo aberto de $\mathbb{R}$ com $0 \in J \subset I$, $W$ um aberto de $\mathbb{R}^{n+1}$ com $V=\left\{z \in \mathbb{R}^{n}:(0, z) \in W\right\} \neq \emptyset$ e $W \subset I \times \Omega$, então denotaremos por

$$
\mathcal{S}\left(I, \Omega, \Omega^{\prime}, H, f, J, W\right)
$$


o conjunto dos pares $(X, P)$ para os quais são verdadeiras as seguintes asserções:

(i) $X \in \mathcal{G}_{*}(J \times V ; \Omega) \quad$ e $P \in \mathcal{G}_{*}\left(J \times V ; \Omega^{\prime}\right)$;

(ii) $(X, P)$ é uma solução do sistema:

$$
\begin{aligned}
& \frac{\partial X}{\partial s}=\frac{\partial H}{\partial p} \circ(\pi, X, P) \quad \text { em } \mathcal{G}\left(J \times V ; \mathbb{R}^{n}\right) \\
& \frac{\partial P}{\partial s}=-\frac{\partial H}{\partial x} \circ(\pi, X, P) \quad \text { em } \mathcal{G}\left(J \times V ; \mathbb{R}^{n}\right) \text {; }
\end{aligned}
$$

(iii) $(X, P)$ satisfaz as condições:

$$
\begin{aligned}
& \left.X\right|_{\{0\} \times V}=1_{V} \quad \text { em } \quad \mathcal{G}\left(V ; \mathbb{R}^{n}\right), \\
& \left.P\right|_{\{0\} \times V}=\left.\nabla f\right|_{V} \quad \text { em } \\
& \mathcal{G}\left(V ; \mathbb{R}^{n}\right) ;
\end{aligned}
$$

(iv) a função $Y=(\pi, X)$ pertence a $\mathcal{G}_{*}(J \times V ; W)$ e é uma aplicação inversível.

O sistema dado em (ii) é chamado, na Teoria Clássica, de sistema Hamiltoniano.

A solução que encontramos para o problema $\mathbf{H J}$ é o seguinte teorema:

2.1.2 Teorema. Sejam I um intervalo aberto de $\mathbb{R}$ com $0 \in I, \Omega$ e $\Omega^{\prime}$ abertos de $\mathbb{R}^{n}, H \in \mathcal{G}\left(I \times \Omega \times \Omega^{\prime} ; \mathbb{R}\right)$ e $f \in \mathcal{G}(\Omega ; \mathbb{R})$. Se $J$ é um intervalo aberto de $\mathbb{R}$ com $0 \in J \subset I, W$ um aberto de $\mathbb{R}^{n+1}$ com $V=\left\{z \in \mathbb{R}^{n}:(0, z) \in W\right\} \neq \emptyset$ e $W \subset I \times \Omega$ e $\mathcal{S}\left(I, \Omega, \Omega^{\prime}, H, f, J, W\right) \neq \emptyset$, então o problema $\mathbf{H J}$ tem uma solução em $\mathcal{G}(W ; \mathbb{R})$.

Demonstração. Denotaremos por $(s, r)=\left(s, r_{1}, \ldots, r_{n}\right)$ um ponto genérico de $J \times V$ e por $(t, x, p)=\left(t, x_{1}, \ldots, x_{n}, p_{1}, \ldots, p_{n}\right)$ um ponto genérico de $I \times \Omega \times \Omega^{\prime}$.

Sejam $X=\left(X_{1}, \ldots, X_{n}\right)$ e $P=\left(P_{1}, \ldots, P_{n}\right)$ tais que $(X, P) \in \mathcal{S}\left(I, \Omega, \Omega^{\prime}, H, f, J, W\right)$, $\hat{f}$ um representante de $f, \hat{l}$ um representante da função generalizada

$$
-H \circ(\pi, X, P)+\sum_{j=1}^{n}\left(\frac{\partial H}{\partial p_{j}} \circ(\pi, X, P)\right) P_{j},
$$

que pode ser definida por 2.1.1.i, $\widehat{U} \in \mathcal{E}_{M}[J \times V ; \mathbb{R}]$ definida por

$$
\widehat{U}\left(\varepsilon, s, r_{1}, \ldots, r_{n}\right)=\widehat{f}\left(\varepsilon, r_{1}, \ldots, r_{n}\right)+\int_{0}^{s} \hat{l}\left(\varepsilon, t, r_{1}, \ldots, r_{n}\right) d t
$$


e seja $U$ a classe de $\widehat{U}$ em $\mathcal{G}(J \times V ; \mathbb{R})$. Então temos que

$$
\begin{aligned}
& \frac{\partial U}{\partial \mathcal{S}}=-H \circ(\pi, X, P)+\sum_{j=1}^{n}\left(\frac{\partial H}{\partial p_{j}} \circ(\pi, X, P)\right) P_{j} ; \\
& \left.U\right|_{\{0\} \times V}=\left.f\right|_{V} \text { em } \mathcal{G}(V ; \mathbb{R}) .
\end{aligned}
$$

Como $(X, P) \in \mathcal{S}\left(I, \Omega, \Omega^{\prime}, H, f, J, W\right)$ sabemos que

$$
\begin{aligned}
& X \in \mathcal{G}_{*}(J \times V ; \Omega) \text { e } P \in \mathcal{G}_{*}\left(J \times V ; \Omega^{\prime}\right) \\
& \frac{\partial X}{\partial s}=\frac{\partial H}{\partial p} \circ(\pi, X, P) \quad \text { em } \mathcal{G}\left(J \times V ; \mathbb{R}^{n}\right) \\
& \frac{\partial P}{\partial s}=-\frac{\partial H}{\partial x} \circ(\pi, X, P) \quad \text { em } \mathcal{G}\left(J \times V ; \mathbb{R}^{n}\right) \\
& \left.X\right|_{\{0\} \times V}=1_{V} \quad \text { em } \mathcal{G}\left(V ; \mathbb{R}^{n}\right) ; \\
& \left.P\right|_{\{0\} \times V}=\left.\nabla f\right|_{V} \quad \text { em } \mathcal{G}\left(V ; \mathbb{R}^{n}\right) ; \\
& Y=(\pi, X) \in \mathcal{G}_{*}(J \times V ; W) \text { e é uma aplicação inversível. }
\end{aligned}
$$

Seja $u=U \circ Y^{-1}$. Provaremos que

$$
\begin{aligned}
& \left.u\right|_{\{0\} \times V}=\left.f\right|_{V} \quad \text { em } \mathcal{G}(V ; \mathbb{R}) \\
& \left(\frac{\partial u}{\partial x_{1}}, \ldots, \frac{\partial u}{\partial x_{n}}\right) \in \mathcal{G}_{*}\left(W ; \Omega^{\prime}\right) ; \\
& \frac{\partial u}{\partial t}+H \circ\left(\pi, \pi_{1}, \ldots, \pi_{n}, \frac{\partial u}{\partial x_{1}}, \ldots, \frac{\partial u}{\partial x_{n}}\right)=0 \text { em } \mathcal{G}(W ; \mathbb{R}),
\end{aligned}
$$

e assim $u$ será uma solução para o problema $\mathbf{H J}$ em $\mathcal{G}(W ; \mathbb{R})$.

Seja $h=\left(h_{0}, h_{1}, \ldots, h_{n}\right)=Y^{-1} \in \mathcal{G}_{*}(W ; J \times V)$. Então por 1.1.25.II e 1.1.26, temos que

e portanto

$$
\begin{aligned}
\left(\left.\pi\right|_{\{0\} \times V},\left.\pi_{1}\right|_{\{0\} \times V}, \ldots,\left.\pi_{n}\right|_{\{0\} \times V}\right) & =\left.\left(\pi, \pi_{1}, \ldots, \pi_{n}\right)\right|_{\{0\} \times V}=\left.(Y \circ h)\right|_{\{0\} \times V} \\
& =\left.(\pi \circ h, X \circ h)\right|_{\{0\} \times V} \\
& =\left(\left.h_{0}\right|_{\{0\} \times V}, X \circ\left(\left.h\right|_{\{0\} \times V}\right)\right),
\end{aligned}
$$

$$
\begin{aligned}
& \left.h_{0}\right|_{\{0\} \times V}=\left.\pi\right|_{\{0\} \times V}=0 \text { em } \mathcal{G}(V ; \mathbb{R}) ; \\
& \left(\left.\pi_{1}\right|_{\{0\} \times V}, \ldots,\left.\pi_{n}\right|_{\{0\} \times V}\right)=X \circ\left(\left.h\right|_{\{0\} \times V}\right) \text { em } \mathcal{G}\left(V ; \mathbb{R}^{n}\right),
\end{aligned}
$$


e assim, por (6) e 1.1.27, temos que

$$
\begin{aligned}
\left(\left.\pi_{1}\right|_{\{0\} \times V}, \ldots,\left.\pi_{n}\right|_{\{0\} \times V}\right) & =X \circ\left(\left.h\right|_{\{0\} \times V}\right) \\
& =X \circ\left(\left(\left.h_{0}\right|_{\{0\} \times V},\left.h_{1}\right|_{\{0\} \times V}, \ldots,\left.h_{n}\right|_{\{0\} \times V}\right)\right) \\
& =X \circ\left(0,\left.h_{1}\right|_{\{0\} \times V}, \ldots,\left.h_{n}\right|_{\{0\} \times V}\right) \\
& =\left.X\right|_{\{0\} \times V} \circ\left(\left.h_{1}\right|_{\{0\} \times V}, \ldots,\left.h_{n}\right|_{\{0\} \times V}\right) \\
& =\left(\left.i_{1}\right|_{\{0\} \times V}, \ldots,\left.h_{n}\right|_{\{0\} \times V}\right) .
\end{aligned}
$$

Portanto, de (2), 1.1.25.II e de 1.1.27, temos que

$$
\begin{aligned}
\left.u\right|_{\{0\} \times V} & =\left.\left(U \circ Y^{-1}\right)\right|_{\{0\} \times V}=\left.(U \circ h)\right|_{\{0\} \times V}=U \circ\left(\left.h\right|_{\{0\} \times V}\right) \\
& =U \circ\left(\left.h_{0}\right|_{\{0\} \times V},\left.h_{1}\right|_{\{0\} \times V}, \ldots,\left.h_{n}\right|_{\{0\} \times V}\right) \\
& =U \circ\left(0,\left.\pi_{1}\right|_{\{0\} \times V}, \ldots,\left.\pi_{n}\right|_{\{0\} \times V}\right) \\
& =\left.U\right|_{\{0\} \times V} \circ 1_{V}=\left.U\right|_{\{0\} \times V}=\left.f\right|_{V},
\end{aligned}
$$

o que prova (8).

Como $u=U \circ Y^{-1}$ temos que $U=u \circ Y$, e assim

$$
\begin{aligned}
& \frac{\partial U}{\partial s}=\frac{\partial u}{\partial t} \circ Y+\sum_{j=1}^{n}\left(\frac{\partial u}{\partial x_{j}} \circ Y\right) \frac{\partial X_{j}}{\partial s} \\
& \frac{\partial U}{\partial r_{i}}=\sum_{j=1}^{n}\left(\frac{\partial u}{\partial x_{j}} \circ Y\right) \frac{\partial X_{j}}{\partial r_{i}}, \text { para todo } 1 \leq i \leq n .
\end{aligned}
$$

De (1), (4) e (11) concluímos que

$$
\left(\frac{\partial u}{\partial t} \circ Y\right)+H \circ(\pi, X, P)+\sum_{j=1}^{n}\left(\frac{\partial u}{\partial x_{j}} \circ Y-P_{j}\right) \frac{\partial X_{j}}{\partial s}=0 .
$$

Provaremos a seguir que

$$
\frac{\partial U}{\partial r_{i}}=\sum_{j=1}^{n} P_{j} \frac{\partial X_{j}}{\partial r_{i}}, \text { para todo } 1 \leq i \leq n .
$$

Fixemos $i \in\{1,2, \ldots, n\}$, e seja

$$
g=\frac{\partial U}{\partial r_{i}}-\sum_{j=1}^{n} P_{j} \frac{\partial X_{j}}{\partial r_{i}} \quad \text { em } \mathcal{G}(J \times V ; \mathbb{R}) .
$$

Usando (1), (4) e (5), temos que 


$$
\begin{aligned}
\frac{\partial g}{\partial s}= & \frac{\partial^{2} U}{\partial s \partial r_{i}}-\sum_{j=1}^{n} P_{j} \frac{\partial^{2} X_{j}}{\partial s \partial r_{i}}-\sum_{j=1}^{n} \frac{\partial P_{j}}{\partial s} \frac{\partial X_{j}}{\partial r_{i}} \\
= & \frac{\partial}{\partial r_{i}}\left(-H \circ(\pi, X, P)+\sum_{j=1}^{n}\left(\frac{\partial H}{\partial p_{j}} \circ(\pi, X, P)\right) P_{j}\right)-\sum_{j=1}^{n} P_{j} \frac{\partial}{\partial r_{i}}\left(\frac{\partial H}{\partial p_{j}} \circ(\pi, X, P)\right)+ \\
& +\sum_{j=1}^{n}\left(\frac{\partial H}{\partial x_{j}} \circ(\pi, X, P)\right) \frac{\partial X_{j}}{\partial r_{i}} \\
= & \frac{\partial}{\partial r_{i}}(-H \circ(\pi, X, P))+\sum_{j=1}^{n} \frac{\partial}{\partial r_{i}}\left(\frac{\partial H}{\partial p_{j}} \circ(\pi, X, P)\right) P_{j}+\sum_{j=1}^{n}\left(\frac{\partial H}{\partial p_{j}} \circ(\pi, X, P)\right) \frac{\partial P_{j}}{\partial r_{i}}+ \\
& -\sum_{j=1}^{n} P_{j} \frac{\partial}{\partial r_{i}}\left(\frac{\partial H}{\partial p_{j}} \circ(\pi, X, P)\right)+\sum_{j=1}^{n}\left(\frac{\partial H}{\partial x_{j}} \circ(\pi, X, P)\right) \frac{\partial X_{j}}{\partial r_{i}},
\end{aligned}
$$

e portanto $\frac{\partial g}{\partial s}=0$ em $\mathcal{G}(J \times V ; \mathbb{R})$.

De (2), (6), (7) e 1.1.25.III temos que

$$
\begin{aligned}
\left.g\right|_{\{0\} \times V} & =\left.\frac{\partial U}{\partial r_{i}}\right|_{\{0\} \times V}-\left.\left.\sum_{j=1}^{n} P_{j}\right|_{\{0\} \times V} \frac{\partial X_{j}}{\partial r_{i}}\right|_{\{0\} \times V} \\
& =\left.\frac{\partial f}{\partial r_{i}}\right|_{V}-\left.\sum_{j=1}^{n} \frac{\partial f}{\partial r_{j}}\right|_{V} \frac{\partial}{\partial r_{i}}\left(\left.X_{j}\right|_{\{0\} \times V}\right) \\
& =\left.\frac{\partial f}{\partial r_{i}}\right|_{V}-\left.\frac{\partial f}{\partial r_{i}}\right|_{V}=0 .
\end{aligned}
$$

Como $\frac{\partial g}{\partial s}=0$ em $\mathcal{G}(J \times V ; \mathbb{R})$ e $\left.g\right|_{\{0\} \times V}=0$ em $\mathcal{G}(V ; \mathbb{R})$ concluímos, por 1.1.15, que $g=0$ em $\mathcal{G}(J \times V ; \mathbb{R})$, e assim (14) é verdadeira.

De (12), (13) e (14) obtemos o seguinte sistema:

$$
\begin{aligned}
& {\left[\left(\frac{\partial u}{\partial t} \circ Y\right)+H \circ(\pi, X, P)\right]+\left[\left(\frac{\partial u}{\partial x_{1}} \circ Y\right)-P_{1}\right] \frac{\partial X_{1}}{\partial s}+\ldots+\left[\left(\frac{\partial u}{\partial x_{n}} \circ Y\right)-P_{n}\right] \frac{\partial X_{n}}{\partial s}=0} \\
& {\left[\left(\frac{\partial u}{\partial x_{1}} \circ Y\right)-P_{1}\right] \frac{\partial X_{1}}{\partial r_{1}}+\ldots+\left[\left(\frac{\partial u}{\partial x_{n}} \circ Y\right)-P_{n}\right] \frac{\partial X_{n}}{\partial r_{1}}=0} \\
& {\left[\left(\frac{\partial u}{\partial x_{1}} \circ Y\right)-P_{1}\right] \frac{\partial X_{1}}{\partial r_{n}}+\ldots+\left[\left(\frac{\partial u}{\partial x_{n}} \circ Y\right)-P_{n}\right] \frac{\partial X_{n}}{\partial r_{n}}=0}
\end{aligned}
$$

Usando que $Y$ é uma aplicação inversível temos, por 1.2.12, que $J Y$ tem inverso 
multiplicativo em $\mathcal{G}(J \times V ; \mathbb{R})$, e assim por 1.1.34 concluímos que o sistema acima admite somente a solução trivial, isto é,

$$
\begin{aligned}
& \frac{\partial u}{\partial t} \circ Y=-H \circ(\pi, X, P) \\
& \frac{\partial u}{\partial x_{j}} \circ Y=P_{j}, \quad \text { para todo } 1 \leq j \leq n .
\end{aligned}
$$

Portanto, usando (3) e que $Y^{-1} \in \mathcal{G}_{*}(W ; J \times V)$ temos, por 1.1 .26 e 1.1 .28 , que

$$
\left(\frac{\partial u}{\partial x_{1}}, \ldots, \frac{\partial u}{\partial x_{n}}\right)=P \circ Y^{-1} \in \mathcal{G}_{*}\left(W ; \Omega^{\prime}\right),
$$

o que prova (9). Notemos também que

o que prova $(10) \cdot / /$

$$
\begin{aligned}
\frac{\partial u}{\partial t} & =-H \circ(\pi, X, P) \circ Y^{-1}=-H \circ\left(\pi, X, \frac{\partial u}{\partial x_{1}} \circ Y, \ldots, \frac{\partial u}{\partial x_{n}} \circ Y\right) \circ Y^{-1} \\
& =-H \circ\left(Y, \frac{\partial u}{\partial x_{1}} \circ Y, \ldots, \frac{\partial u}{\partial x_{n}} \circ Y\right) \circ Y^{-1} \\
& =-H \circ\left(\pi, \pi_{1}, \ldots, \pi_{n}, \frac{\partial u}{\partial x_{1}}, \ldots, \frac{\partial u}{\partial x_{n}}\right),
\end{aligned}
$$

Como uma conseqüência da demonstração do resultado anterior temos o seguinte:

2.1.3 Proposição. Sejam $I, \Omega, \Omega^{\prime}, H, f, J, V$ e $W$ como em 2.1.2. Se $(X, P) \in$ $\mathcal{S}\left(I, \Omega, \Omega^{\prime}, H, f, J, W\right), \quad Y=(\pi, X), \quad P=\left(P_{1}, \ldots, P_{n}\right)$ e $U \in \mathcal{G}(J \times V ; \mathbb{R})$ é tal que

$$
\frac{\partial U}{\partial s}=-H \circ(\pi, X, P)+\sum_{j=1}^{n}\left(\frac{\partial H}{\partial p_{j}} \circ(\pi, X, P)\right) P_{j} \quad \text { e }\left.\quad U\right|_{\{0\} \times V}=\left.f\right|_{V},
$$

onde $(t, x, p)=\left(t, x_{1}, \ldots, x_{n}, p_{1}, \ldots, p_{n}\right)$ denota um ponto genérico de $I \times \Omega \times \Omega^{\prime}$, entâo

(I) $u=U \circ Y^{-1}$ é uma solução para o problema $\mathbf{H J}$ em $\mathcal{G}(W ; \mathbb{R})$;

(II) denotando por $(t, x)=\left(t, x_{1}, \ldots, x_{n}\right)$ um ponto genérico de $W$, tem-se que

$$
\left(\frac{\partial u}{\partial x_{1}}, \ldots, \frac{\partial u}{\partial x_{n}}\right)=P \circ Y^{-1} . / /
$$


Veremos na seção 3.3 que, sob certas condições,

$$
\operatorname{card} \mathcal{S}\left(I, \Omega, \Omega^{\prime}, H, f, J, W\right) \leq 1
$$

Com o resultado anterior vimos que para obter uma solução para o problema $\mathbf{H J}$ em $\mathcal{G}(W ; \mathbb{R})$ é suficiente ter $\mathcal{S}\left(I, \Omega, \Omega^{\prime}, H, f, J, W\right) \neq \emptyset$. Veremos a seguir que isto sempre ocorre quando $H$ e $f$ são funções $\mathcal{C}^{\infty}$. Aqui estamos usando a seguinte notação:

Se $\Omega$ é um aberto de $\mathbb{R}^{n}, p \in \mathbb{N}^{*}, l=\left(l_{1}, \ldots, l_{p}\right)$ é tal que $l_{i} \in \mathcal{C}^{\infty}(\Omega ; \mathbb{R})$ para todo $1 \leq i \leq p$ e $\hat{g}$ é a função moderada definida em $] 0,1] \times \Omega$ por $\widehat{g}(\varepsilon, x)=l(x)$, então ainda denotaremos por $l$ a classe $\operatorname{de} \hat{g} \operatorname{em~} \mathcal{G}\left(\Omega ; \mathbb{R}^{p}\right)$.

2.1.4 Proposição. Sejam I um intervalo aberto de $\mathbb{R}$ com $0 \in I, \Omega$ e $\Omega^{\prime}$ abertos de $\mathbb{R}^{n}, H \in \mathcal{C}^{\infty}\left(I \times \Omega \times \Omega^{\prime} ; \mathbb{R}\right)$ e $f \in \mathcal{C}^{\infty}(\Omega ; \mathbb{R})$. Então dado $x_{o} \in \Omega$ existem $V$ um aberto de $\mathbb{R}^{n}$ com $x_{o} \in V \subset \Omega, W$ um aberto de $\mathbb{R}^{n+1}$ com $W \subset I \times \Omega$ e de modo que $V=\left\{z \in \mathbb{R}^{n}:(0, z) \in W\right\} \neq \emptyset$ e existe um intervalo aberto $J$ de $\mathbb{R}$ com $0 \in J \subset I$ tais que $\mathcal{S}\left(I, \Omega, \Omega^{\prime}, H, f, J, W\right) \neq \emptyset$.

Demonstração. Denotaremos por $(s, r)=\left(s, r_{1}, \ldots, r_{n}\right)$ um ponto genérico de $\mathbb{R}^{n+1}$ e por $(t, x, p)=\left(t, x_{1}, \ldots, x_{n}, p_{1}, \ldots, p_{n}\right)$ um ponto genérico de $\mathbb{R}^{2 n+1}$.

Seja $x_{\circ} \in \Omega$. Então da teoria clássica de equações diferenciais ordinárias, existem $a>0, V_{1}$ aberto de $\mathbb{R}^{n}$ com $x_{o} \in V_{1}$ e uma aplicação $(X, P)$, sendo $X=\left(X_{1}, \ldots, X_{n}\right)$ e $P=\left(P_{1}, \ldots, P_{n}\right)$, definida em $I_{a} \times V_{1} \subset I \times \Omega$ tal que

$$
\begin{aligned}
& X_{i} \quad \text { e } \quad P_{i} \quad \text { pertencem a } \mathcal{C}^{\infty}\left(I_{a} \times V_{1} ; \mathbb{R}\right), \text { para todo } 1 \leq i \leq n ; \\
& X\left(I_{a} \times V_{1}\right) \subset \Omega \quad \text { e } \quad P\left(I_{a} \times V_{1}\right) \subset \Omega^{\prime} ; \\
& \frac{\partial X}{\partial s}(s, r)=\frac{\partial H}{\partial p}(s, X(s, r), P(s, r)) \quad \text { e } \quad \frac{\partial P}{\partial s}(s, r)=-\frac{\partial H}{\partial x}(s, X(s, r), P(s, r)) ; \\
& \left.X\right|_{\{0\} \times V_{1}}=\left.1\right|_{V_{1}} \quad \text { e }\left.\quad P\right|_{\{0\} \times V_{1}}=\left.\nabla f\right|_{V_{1}} .
\end{aligned}
$$

Seja $Y=(\pi, X)$.

Como $\operatorname{det}\left(\mathrm{d} Y_{\left(0, x_{o}\right)}\right)=\operatorname{det}\left(\frac{\partial X_{i}}{\partial r_{j}}\left(0, x_{o}\right)\right)_{1 \leq i, j \leq n}=1 \neq 0$, existem, pelo Teorema da Função Inversa, um aberto $W_{1}$ de $\mathbb{R}^{n+1} \operatorname{com}\left(0, x_{o}\right) \in W_{1} \subset I_{a} \times V_{1}$ e um aberto $W_{2}$ de 
$\mathbb{R}^{n+1}$ com $\left(0, x_{o}\right)=Y\left(0, x_{o}\right) \in W_{2} \subset Y\left(I_{a} \times V_{1}\right)$ tal que $\left.Y\right|_{W_{1}}$ é um difeomorfismo de classe $\mathcal{C}^{\infty}$ sobre $W_{2}$.

Usando que $\left(0, x_{0}\right) \in W_{1}$ existem um intervalo aberto $J$ de $\mathbb{R}$ e um aberto $V$ de $\mathbb{R}^{n}$ tais que $\left(0, x_{o}\right) \in J \times V \subset W_{1} \subset I_{a} \times V_{1}$.

Como $\left.Y\right|_{W_{1}}$ é um difeomorfismo temos que $Y(J \times V)$ é um aberto de $\mathbb{R}^{n+1}$.

Seja $W=Y(J \times V) \subset J \times X(J \times V) \subset I \times \Omega$. Notemos que,

$$
\text { se } \quad A=\left\{z \in \mathbb{R}^{n}:(0, z) \in W\right\}, \quad \text { então } \quad A=V \text {. }
$$

De fato, se $z \in A$, então $(0, z) \in W$ e assim existe $(a, b) \in J \times V$ tal que $(a, X(a, b))=$ $Y(a, b)=(0, z)$, e portanto $a=0$ e $X(0, b)=z$, e assim de (4) concluímos que $z=$ $X(0, b)=b \in V$, e portanto $A \subset V$. Se $z \in V$ temos, por (4), que $Y(0, z)=(0, X(0, z))=$ $(0, z)$ e $\operatorname{assim}(0, z) \in Y(J \times V)=W$, e portanto $V \subset A$.

Usando (1), (2), (3), (4), que $\left.Y\right|_{J \times V}$ é uma aplicação inversível e que $V=\left\{z \in \mathbb{R}^{n}\right.$ : $(0, z) \in W\}$ concluímos que $\left(\left.X\right|_{J \times V},\left.P\right|_{J \times V}\right) \in \mathcal{S}\left(I, \Omega, \Omega^{\prime}, H, f, J, W\right)$. //

Para dar exemplos de funções $H$ e $f$ generalizadas para as quais

$$
\mathcal{S}\left(I, \Omega, \Omega^{\prime}, H, f, J, W\right) \neq \emptyset
$$

(e portanto o problema HJ admite uma solução), precisamos, em primeiro lugar, resolver o sistema 2.1.1.ii com a condição 2.1.1.iii e depois verificar se 2.1.1.iv está satisfeita. Daremos a seguir alguns exemplos onde $\frac{\partial H}{\partial x}$ é um vetor generalizado ou $\frac{\partial H}{\partial x}=0$ (e portanto $\Omega=\mathbb{R}^{n}$ ), pois neste caso o sistema 2.1.1.ii com a condição 2.1.1.iii é mais fácil de resolver, e assim basta garantir que 2.1.1.iv é verdadeira. Veremos também que a solução encontrada para o problema HJ possui, sob algumas condições, uma certa propriedade. Soluções para o problema HJ com essa propriedade serão estudadas na seção 3.3.

2.1.5 Proposição. Sejam I um intervalo aberto de $\mathbb{R}$ com $0 \in I, f \in \mathcal{G}\left(\mathbb{R}^{n} ; \mathbb{R}\right)$ e $\mu=\left(\mu_{1}, \ldots, \mu_{n}\right) \in\left(\mathcal{E}_{M}(\mathbb{R})\right)^{n}$ tais que

(i) $\nabla f \in \mathcal{G}_{*}\left(\mathbb{R}^{n} ; \mathbb{R}^{n}\right)$; 
(ii) $\mu$ é uma aplicação limitada, isto é, $\mu_{i}$ é uma função limitada no sentido usual, para todo $1 \leq i \leq n$.

Denotando por $r=\left(r_{1}, \ldots, r_{n}\right)$ um ponto genérico de $\mathbb{R}^{n} e(t, p)=\left(t, p_{1}, \ldots, p_{n}\right)$ um ponto genérico de $\mathbb{R}^{n+1}$ tem-se que, se $\hat{f}$ é um representante de $f, \widehat{h} \in \mathcal{E}_{M}\left[I \times \mathbb{R}^{n} ; \mathbb{R}\right], M>0$ e $\tau \in] 0,1]$ são tais que

(iii) $\left|\frac{\partial^{2} \hat{f}}{\partial r_{j} \partial r_{i}}(\varepsilon, r)\right| \leq M, \quad$ para todo $\left.(\varepsilon, r) \in\right] 0, \tau\left[\times \mathbb{R}^{n} \quad\right.$ e $1 \leq i, j \leq n$;

(iv) $\max \left\{\left|\frac{\partial \widehat{h}}{\partial p_{i}}(\varepsilon, t, p)\right|,\left|\frac{\partial^{2} \widehat{h}}{\partial t \partial p_{i}}(\varepsilon, t, p)\right|,\left|\frac{\partial^{2} \widehat{h}}{\partial p_{j} \partial p_{i}}(\varepsilon, t, p)\right|\right\} \leq M$, para todo $(\varepsilon, t, p) \in] 0, \tau\left[\times I \times \mathbb{R}^{n}\right.$ e $1 \leq i, j \leq n$,

e se $H$ é a classe em $\mathcal{G}\left(I \times \mathbb{R}^{n} \times \mathbb{R}^{n} ; \mathbb{R}\right)$ da função moderada $\widehat{H}$ definida em $\left.] 0,1\right] \times I \times$ $\mathbb{R}^{n} \times \mathbb{R}^{n}$ por

$$
\widehat{H}\left(\varepsilon, t, x_{1}, \ldots, x_{n}, p_{1}, \ldots, p_{n}\right)=\widehat{h}\left(\varepsilon, t, p_{1}, \ldots, p_{n}\right)+\sum_{i=1}^{n} \mu_{i}(\varepsilon) x_{i}
$$

então existe $a>0$ com $\overline{I_{a}} \subset I$ e $\mathcal{S}\left(I, \mathbb{R}^{n}, \mathbb{R}^{n}, H, f, I_{a}, I_{a} \times \mathbb{R}^{n}\right) \neq \emptyset$, e em conseqüência existe $u \in \mathcal{G}\left(I_{a} \times \mathbb{R}^{n} ; \mathbb{R}\right)$ tal que

(I) u é uma solução para o problema $\mathbf{H J}$ em $\mathcal{G}\left(I_{a} \times \mathbb{R}^{n} ; \mathbb{R}\right)$.

Se $(t, x)=\left(t, x_{1}, \ldots, x_{n}\right)$ denota um ponto genérico de $I_{a} \times \mathbb{R}^{n}$, então u também satisfaz

(II) existem $\widehat{v}=\left(\widehat{v}_{1}, \ldots, \widehat{v}_{n}\right)$ um representante de $\left(\frac{\partial u}{\partial x_{1}}, \ldots, \frac{\partial u}{\partial x_{n}}\right)$ e $\bar{M}>0$ tais que $\left|\frac{\partial \hat{v}_{i}}{\partial x_{j}}(\varepsilon, t, x)\right| \leq \bar{M}, \quad$ para todo $\left.(\varepsilon, t, x) \in\right] 0, \tau\left[\times I_{a} \times \mathbb{R}^{n} \quad e \quad 1 \leq i, j \leq n\right.$.

Demonstração. Provaremos, em primeiro lugar, que $\mathcal{S}\left(I, \mathbb{R}^{n}, \mathbb{R}^{n}, H, f, I_{a}, I_{a} \times \mathbb{R}^{n}\right) \neq \emptyset$ para algum $a>0$, e com o auxílio de 2.1 .3 obteremos (I).

Denotaremos por $(t, x, p)=\left(t, x_{1}, \ldots, x_{n}, p_{1}, \ldots, p_{n}\right)$ um ponto genérico de $\mathbb{R}^{2 n+1}$ e por $(s, r)=\left(s, r_{1}, \ldots, r_{n}\right)$ ou por $(t, p)=\left(t, p_{1}, \ldots, p_{n}\right)$ um ponto genérico de $\mathbb{R}^{n+1}$.

Sejam $\widehat{X}=\left(\widehat{X}_{1}, \ldots, \widehat{X}_{n}\right)$ a aplicação definida em $\left.] 0,1\right] \times I \times \mathbb{R}^{n}$ por 


$$
\widehat{X}(\varepsilon, s, r)=r+\int_{0}^{s} \frac{\partial \widehat{H}}{\partial p}(\varepsilon, t, r, \nabla \widehat{f}(\varepsilon, r)-\mu(\varepsilon) t) d t,
$$

que por (i) e (ii) é moderada, e $\widehat{P}=\left(\widehat{P}_{1}, \ldots, \widehat{P}_{n}\right)$ e $\widehat{g}=\left(\widehat{g}_{1}, \ldots, \widehat{g}_{n}\right)$ as aplicações moderadas definidas em $] 0,1] \times I \times \mathbb{R}^{n}$ por

$$
\widehat{P}(\varepsilon, s, r)=\nabla \widehat{f}(\varepsilon, r)-\mu(\varepsilon) s
$$

e

$$
\widehat{g}(\varepsilon, s, r)=\widehat{X}(\varepsilon, s, r)-r=\int_{0}^{s} \frac{\partial \widehat{H}}{\partial p}(\varepsilon, t, r, \nabla \widehat{f}(\varepsilon, r)-\mu(\varepsilon) t) d t .
$$

Notemos que

$$
\begin{aligned}
& \frac{\partial \widehat{g}_{i}}{\partial s}(\varepsilon, s, r)=\frac{\partial \widehat{h}}{\partial p_{i}}(\varepsilon, s, \nabla \widehat{f}(\varepsilon, r)-\mu(\varepsilon) s) ; \\
& \frac{\partial^{2} \widehat{g}_{i}}{\partial s^{2}}(\varepsilon, s, r)=\frac{\partial^{2} \widehat{h}}{\partial t \partial p_{i}}(\varepsilon, s, \nabla \widehat{f}(\varepsilon, r)-\mu(\varepsilon) s)-\sum_{j=1}^{n} \frac{\partial^{2} \widehat{h}}{\partial p_{j} \partial p_{i}}(\varepsilon, s, \nabla \widehat{f}(\varepsilon, r)-\mu(\varepsilon) s) \mu_{j}(\varepsilon) ; \\
& \frac{\partial^{2} \widehat{g}_{i}}{\partial r_{k} \partial s}(\varepsilon, s, r)=\sum_{j=1}^{n} \frac{\partial^{2} \widehat{h}}{\partial p_{j} \partial p_{i}}(\varepsilon, s, \nabla \widehat{f}(\varepsilon, r)-\mu(\varepsilon) s) \frac{\partial^{2} \widehat{f}}{\partial r_{k} \partial r_{j}}(\varepsilon, r) ;
\end{aligned}
$$

para todo $(\varepsilon, s, r) \in] 0,1] \times I \times \mathbb{R}^{n}$ e $1 \leq i, k \leq n$, e assim, usando (iii) e (iv), temos que

$$
\begin{aligned}
& \left|\frac{\partial \widehat{g}_{i}}{\partial s}(\varepsilon, s, r)\right| \leq M \\
& \left.\left.\left|\frac{\partial^{2} \widehat{g}_{i}}{\partial s^{2}}(\varepsilon, s, r)\right| \leq M+n M\{\|\mu(\varepsilon)\|: \varepsilon \in] 0,1\right]\right\} ; \\
& \left|\frac{\partial^{2} \widehat{g}_{i}}{\partial r_{k} \partial s}(\varepsilon, s, r)\right| \leq n M^{2},
\end{aligned}
$$

para todo $(\varepsilon, s, r) \in] 0, \tau\left[\times I \times \mathbb{R}^{n}\right.$ e $1 \leq i, k \leq n$.

Sejam $\hat{Y}$ a aplicação moderada definida em $] 0,1] \times I \times \mathbb{R}^{n}$ por $\hat{Y}=(\widehat{\pi}, \widehat{X})$ e $Y$ a classe de $\hat{Y}$ em $\mathcal{G}\left(I \times \mathbb{R}^{n} ; \mathbb{R}^{n+1}\right)$.

Como $\widehat{g}_{i}(\varepsilon, 0, r)=0$, para todo $\left.\left.(\varepsilon, r) \in\right] 0,1\right] \times \mathbb{R}^{n}$ e $1 \leq i \leq n, \mu$ é uma função limitada e valem (1), (2) e (3) temos, por 1.2.22, que existe $a>0$ tal que $\overline{I_{a}} \subset I$,

$\left.Y\right|_{I_{a} \times R^{n}} \in \mathcal{G}_{*}\left(I_{a} \times \mathbb{R}^{n} ; I_{a} \times \mathbb{R}^{n}\right) ;$

$\left.Y\right|_{I_{a} \times R^{n}}$ é uma aplicação inversível;

$$
c=\inf \{|J \hat{Y}(\varepsilon, s, r)|:(\varepsilon, s, r) \in] 0, \tau\left[\times I_{a} \times \mathbb{R}^{n}\right\}>0 ;
$$


existe um representante $\widehat{\Gamma}=\left(\widehat{\Gamma}_{0}, \widehat{\Gamma}_{1}, \ldots, \widehat{\Gamma}_{n}\right)$ de $\left(\left.Y\right|_{I_{a} \times R^{n}}\right)^{-1}$ tal que

$$
\left.\widehat{\Gamma}(\varepsilon, .)=\left(\left.\hat{Y}(\varepsilon, .)\right|_{I_{a} \times R^{n}}\right)^{-1}, \text { para todo } \varepsilon \in\right] 0, \tau[;
$$

existe $M_{1}>0$ tal que, se $0 \leq i \leq n$, então

$\left|\partial^{\alpha} \widehat{\Gamma}_{i}(\varepsilon, t, x)\right| \leq M_{1}$, para todo $\left.(\varepsilon, t, x) \in\right] 0, \tau\left[\times I_{a} \times \mathbb{R}^{n}\right.$ e $\alpha \in \mathbb{N}^{n+1}$ com $|\alpha|=1$.

Sejam $X$ a classe de $\widehat{X}$ e $P$ a classe de $\widehat{P}$ em $\mathcal{G}\left(I \times \mathbb{R}^{n} ; \mathbb{R}^{n}\right)$. Então, por (i), (ii) e (iv), temos que

$$
\left.X\right|_{I_{a} \times R^{n}} \text { e }\left.P\right|_{I_{a} \times R^{n}} \text { pertencem a } \mathcal{G}_{*}\left(I_{a} \times \mathbb{R}^{n} ; \mathbb{R}^{n}\right)
$$

e como $\left(\left.X\right|_{I_{a} \times R^{n}},\left.P\right|_{I_{a} \times R^{n}}\right)$ satisfaz 2.1.1.ii e 2.1.1.iii e

$\left.Y\right|_{I_{a} \times R^{n}}=\left(\left.\pi\right|_{I_{a} \times R^{n}},\left.X\right|_{I_{a} \times R^{n}}\right) \in \mathcal{G}_{*}\left(I_{a} \times \mathbb{R}^{n} ; I_{a} \times \mathbb{R}^{n}\right)$ é uma aplicação inversível, temos que

$$
\left(\left.X\right|_{I_{a} \times R^{n}},\left.P\right|_{I_{a} \times R^{n}}\right) \in \mathcal{S}\left(I, \mathbb{R}^{n}, \mathbb{R}^{n}, H, f, I_{a}, I_{a} \times \mathbb{R}^{n}\right),
$$

e assim, por 2.1.3, temos que $u=U \subset\left(\left.Y\right|_{I_{a} \times R^{n}}\right)^{-1}$ é uma solução para o problema $\mathbf{H J}$ em $\mathcal{G}\left(I_{a} \times \mathbb{R}^{n} ; \mathbb{R}\right)$ e $\left(\frac{\partial u}{\partial x_{1}}, \ldots, \frac{\partial u}{\partial x_{n}}\right)=P$ ○ $\left(\left.Y\right|_{I_{a} \times R^{n}}\right)^{-1}$, onde $U$ é como em $2.1 .3 \mathrm{e}$ $(t, x)=\left(t, x_{1}, \ldots, x_{n}\right)$ denota um ponto genérico de $I_{a} \times \mathbb{R}^{n}$.

Portanto (I) é verdadeira.

Seja $\widehat{v}=\widehat{P} \circ \widehat{\Gamma}$, sendo $\widehat{\Gamma}$ como em (5). Então $\widehat{v}=\left(\widehat{v}_{1}, \ldots, \widehat{v}_{n}\right)$ é um representante de $\left(\frac{\partial u}{\partial x_{1}}, \ldots, \frac{\partial u}{\partial x_{n}}\right)$. Provaremos que $\hat{v}$ satisfaz (II) e assim concluiremos a prova.

Notemos que, se $1 \leq i, j \leq n$, então

$$
\begin{aligned}
\frac{\partial \widehat{v}_{i}}{\partial x_{j}}(\varepsilon, t, x) & =\frac{\partial}{\partial x_{j}}\left(\widehat{P}_{i} \circ \widehat{\Gamma}\right)(\varepsilon, t, x) \\
& =\frac{\partial \hat{P}_{i}}{\partial s}(\varepsilon, \widehat{\Gamma}(\varepsilon, t, x)) \frac{\partial \widehat{\Gamma}_{0}}{\partial x_{j}}(\varepsilon, t, x)+\sum_{k=1}^{n} \frac{\partial \widehat{P}_{i}}{\partial r_{k}}(\varepsilon, \widehat{\Gamma}(\varepsilon, t, x)) \frac{\partial \widehat{\Gamma}_{k}}{\partial x_{j}}(\varepsilon, t, x),
\end{aligned}
$$

e assim

$$
\frac{\partial \widehat{v}_{i}}{\partial x_{j}}(\varepsilon, t, x)=-\mu_{i}(\varepsilon) \frac{\partial \widehat{\Gamma}_{0}}{\partial x_{j}}(\varepsilon, t, x)+\sum_{k=1}^{n} \frac{\partial^{2} \widehat{f}}{\partial r_{k} \partial r_{i}}(\varepsilon, \widehat{\Gamma}(\varepsilon, t, x)) \frac{\partial \widehat{\Gamma}_{k}}{\partial x_{j}}(\varepsilon, t, x),
$$

para todo $(\varepsilon, t, x) \in] 0, \tau\left[\times I_{a} \times \mathbb{R}^{n}\right.$.

Usando (6), (7), (ii) e (iii) concluímos (II). // 
A seguir apresentamos algumas funções que satisfazem as hipóteses de 2.1.5.

2.1.6 Exemplo. Sejam $b>0,1 \leq j \leq n, \nu_{j}$ e $\tilde{\nu}_{j}$ funções definidas em ]0,1] e com valores em $\mathbb{R}$ e $\varphi_{j}, \psi_{j}$ e $\Phi_{j}$ funçôes pertencentes a $\mathcal{C}^{\infty}(\mathbb{R} ; \mathbb{R})$ tais que $\nu_{j}, \tilde{\nu}_{j}, \varphi_{j}, \psi_{j}$, $\psi_{j}^{\prime}$ e $\Phi_{j}$ são funções limitadas em $\mathbb{R}$. Então a função moderada $\hat{f}$ definida em $\left.] 0,1\right] \times \mathbb{R}^{n}$ por uma das funções

$$
\begin{aligned}
& \hat{l}_{1}\left(\varepsilon, r_{1}, \ldots, r_{n}\right)=\sum_{j=1}^{n} \int_{0}^{r_{j}}\left(\int_{0}^{\lambda} \varphi_{j}\left(\nu_{j}(\varepsilon) y\right) d y\right) d \lambda ; \\
& \hat{l}_{2}\left(\varepsilon, r_{1}, \ldots, r_{n}\right)=\sum_{j=1}^{n} \nu_{j}(\varepsilon) \int_{0}^{r_{j}}\left(\int_{0}^{\lambda} \varphi_{j}(y) d y\right) d \lambda ;
\end{aligned}
$$

satisfaz 2.1.5.iii, a classe de $\hat{f}$ em $\mathcal{G}\left(\mathbb{R}^{n} ; \mathbb{R}\right)$ satisfaz 2.1.5.i e a função moderada $\hat{h}$ definida em $] 0,1] \times I_{b} \times \mathbb{R}^{n}$ por

$$
\widehat{h}\left(\varepsilon, t, p_{1}, \ldots, p_{n}\right)=\sum_{j=1}^{n} t \int_{0}^{p_{j}} \psi_{j}\left(\tilde{\nu}_{j}(\varepsilon) y\right) d y
$$

ou por, se $\psi_{j}^{\prime \prime}$ é uma função limıitada para todo $1 \leq j \leq n$,

$$
\widehat{h}\left(\varepsilon, t, p_{1}, \ldots, p_{n}\right)=\sum_{i=1}^{n} \psi_{j}\left(\tilde{\nu}_{j}(\varepsilon)\left(t+p_{j}\right)\right)
$$

ou por, se $\left.\left.\inf \left\{\left|\tilde{\nu}_{j}(\varepsilon)\right|: \varepsilon \in\right] 0,1\right]\right\}>0$ e $\Phi_{j} \in L_{1}(\mathbb{R})$, para todo $1 \leq j \leq n$,

$$
\widehat{h}\left(\varepsilon, t, p_{1}, \ldots, p_{n}\right)=\sum_{j=1}^{n} t \int_{0}^{p_{j}}\left(\int_{0}^{\lambda} \Phi_{j}\left(\tilde{\nu}_{j}(\varepsilon) y\right) d y\right) d \lambda
$$

satisfaz 2.1.5.iv (substituindo $I$ por $I_{b}$ ).

(As funções $g_{1}(x)=1, g_{2}(x)=\operatorname{arctg} x$ e $g_{3}(x)=\cos x$ podem, por exemplo, ser usadas para $\varphi_{j}$ ou $\psi_{j}$. A função $g_{4}(x)=\operatorname{sen}\left(x^{2}\right)$ pode ser usada para $\varphi_{j}$ e $g_{5}(x)=\exp \left(-x^{2}\right)$ para $\left.\Phi_{j}.\right)$

Quando $I$ é um intervalo aberto de $\mathbb{R}$ com $0 \in I$ e $\frac{\partial H}{\partial x}=0$ em $\mathcal{G}\left(I \times \mathbb{R}^{n} \times \mathbb{R}^{n} ; \mathbb{R}^{n}\right)$, além de 2.1.5 obtivemos, para resolver o problema $\mathbf{H J}$, neste caso, a seguinte proposição: 
2.1.7 Proposição. Sejam I um intervalo aberto de $\mathbb{R}$ com $0 \in I,\left(\widehat{h}_{i}\right)_{1 \leq i \leq n}$ e $\left(\widehat{f}_{i}\right)_{1 \leq i \leq n}$ seqüências finitas de elementos de, respectivamente, $\mathcal{E}_{M}[I \times \mathbb{R} ; \mathbb{R}]$ e $\mathcal{E}_{M}[\mathbb{R} ; \mathbb{R}], \widehat{H}$ a função moderada definida em $] 0,1] \times I \times \mathbb{R}^{n} \times \mathbb{R}^{n}$ por

$$
\widehat{H}\left(\varepsilon, t, x_{1}, \ldots, x_{n}, p_{1}, \ldots, p_{n}\right)=\sum_{i=1}^{n} \widehat{h}_{i}\left(\varepsilon, t, p_{i}\right)
$$

$H$ a classe de $\widehat{H}$ em $\mathcal{G}\left(I \times \mathbb{R}^{n} \times \mathbb{R}^{n} ; \mathbb{R}\right), \hat{f}$ a funçấo moderada definida em $\left.] 0,1\right] \times \mathbb{R}^{n}$ por

$$
\widehat{f}\left(\varepsilon, r_{1}, \ldots, r_{n}\right)=\sum_{i=1}^{n} \widehat{f}_{i}\left(\varepsilon, r_{i}\right),
$$

$f$ a classe de $\hat{f}$ em $\mathcal{G}\left(\mathbb{R}^{n} ; \mathbb{R}\right)$ e $\left.\left.\tau \in\right] 0,1\right]$. Denotando por $(t, y)$ um ponto genérico de $I \times \mathbb{R}$ tem-se que, se

(i) $\nabla f \in \mathcal{G}_{*}\left(\mathbb{R}^{n} ; \mathbb{R}^{n}\right)$;

(ii) existem $\varphi \in \mathcal{C}(I ; \mathbb{R}) \quad e\left(a_{1}, \ldots, a_{n}\right) \in \mathbb{R}^{n}$ tais que

$$
\left.\left|\frac{\partial \hat{h}_{i}}{\partial y}\left(\varepsilon, t, \frac{\partial \hat{f}_{i}}{\partial y}\left(\varepsilon, a_{i}\right)\right)\right| \leq \varphi(t), \quad \text { para todo }(\varepsilon, t) \in\right] 0, \tau[\times I \quad \text { e } \quad 1 \leq i \leq n ;
$$

(iii) existem a e $b$ números reais tais que

$$
\left.a \leq \frac{\partial^{2} \widehat{h}_{i}}{\partial y^{2}}\left(\varepsilon, t, \frac{\partial \widehat{f}_{i}}{\partial y}(\varepsilon, y)\right) \frac{\partial^{2} \widehat{f}_{i}}{\partial y^{2}}(\varepsilon, y) \leq b, \text { para todo }(\varepsilon, t, y) \in\right] 0, \tau[\times I \times \mathbb{R} \text { e } 1 \leq i \leq n
$$

e se a e b são como em (iii) e $\psi$ é a função definida em $\mathbb{R}$ por $\psi(s)=a s$, se $s>0$ e $\psi(s)=b s$, se $s \leq 0$, então dado qualquer intervalo aberto $J$ de $\mathbb{R}$ com $0 \in J \subset$ $\psi^{-1}(]-1, \infty[) \cap I$, tem-se que $\mathcal{S}\left(I, \mathbb{R}^{n}, \mathbb{R}^{n}, H, f, J, J \times \mathbb{R}^{n}\right) \neq \emptyset$, e em conseqüência existe $u \in \mathcal{G}\left(J \times \mathbb{R}^{n} ; \mathbb{R}\right)$ tal que

(I) u é uma solução para o problema $\mathbf{H J}$ em $\mathcal{G}\left(J \times \mathbb{R}^{n} ; \mathbb{R}\right)$.

Denotando por $(t, x)=\left(t, x_{1}, \ldots, x_{n}\right)$ um ponto genérico de $J \times \mathbb{R}^{n}$ tem-se que u também satisfaz

(II) se $\left(\frac{\partial^{2} f}{\partial r_{1}^{2}}, \ldots, \frac{\partial^{2} f}{\partial r_{n}^{2}}\right) \in \mathcal{G}_{*}\left(\mathbb{R}^{n} ; \mathbb{R}^{n}\right)$, então $\frac{\partial^{2} u}{\partial x_{i} \partial x_{j}} \in \mathcal{G}_{*}\left(J \times \mathbb{R}^{n} ; \mathbb{R}\right)$, para todo $1 \leq i, j \leq n$. 
Demonstração. Provaremos, em primeiro lugar, que $\mathcal{S}\left(I, \mathbb{R}^{n}, \mathbb{R}^{n}, H, f, J, J \times \mathbb{R}^{n}\right) \neq \emptyset$ e com o auxílio de 2.1 .3 obteremos (I).

Denotaremos por $(s, r)=\left(s, r_{1}, \ldots, r_{n}\right)$ um ponto genérico de $\mathbb{R}^{n+1}, r=\left(r_{1}, \ldots, r_{n}\right)$ um ponto genérico de $\mathbb{R}^{n}$ e por $(s, y)$ um ponto genérico de $I \times \mathbb{R}$.

Sejam $\widehat{X}=\left(\widehat{X}_{1}, \ldots, \widehat{X}_{n}\right)$ a aplicação definida em $\left.] 0,1\right] \times I \times \mathbb{R}^{n}$ por

$$
\widehat{X}(\varepsilon, s, r)=r+\int_{0}^{s} \frac{\partial \widehat{H}}{\partial p}(\varepsilon, t, r, \nabla \widehat{f}(\varepsilon, r)) d t
$$

que por (i) é moderada, $\widehat{P}=\left(\widehat{P}_{1}, \ldots, \widehat{P}_{n}\right)$ a aplicação moderada definida em $\left.] 0,1\right] \times I \times \mathbb{R}^{n}$ por

$$
\widehat{P}(\varepsilon, s, r)=\nabla \widehat{f}(\varepsilon, r)
$$

e $\hat{g}_{j}$ a função definida em $\left.] 0,1\right] \times I \times \mathbb{R}$ por

$$
\widehat{g}_{j}(\varepsilon, s, y)=\widehat{X}_{j}(\varepsilon, s, r)-r_{j}=\int_{0}^{s} \frac{\partial \widehat{h}_{j}}{\partial y}\left(\varepsilon, t, \frac{\partial \widehat{f}_{j}}{\partial y}(\varepsilon, y)\right) d t,
$$

sendo $1 \leq j \leq n$.

$\operatorname{Sejam} \varphi, a$ e $b$ como em (ii) e (iii).

Notemos que,

$$
\left|\widehat{g}_{i}\left(\varepsilon, s, a_{i}\right)\right| \leq\left|\int_{0}^{s}\right| \frac{\partial \widehat{h}_{i}}{\partial y}\left(\varepsilon, t, \frac{\partial \widehat{f}_{i}}{\partial y}\left(\varepsilon, a_{i}\right)\right)|d t| \leq\left|\int_{0}^{s} \varphi(t) d t\right|,
$$

para todo $(\varepsilon, s) \in] 0, \tau[\times I$ e $1 \leq i \leq n$, e que

$$
\frac{\partial \widehat{g}_{i}}{\partial y}(\varepsilon, s, y)=\int_{0}^{s} \frac{\partial^{2} \widehat{h}_{i}}{\partial y^{2}}\left(\varepsilon, t, \frac{\partial \widehat{f}_{i}}{\partial y}(\varepsilon, y)\right) \frac{\partial^{2} \widehat{f}_{i}}{\partial y^{2}}(\varepsilon, y) d t
$$

para todo $(\varepsilon, s, y) \in] 0, \tau[\times I \times \mathbb{R}$ e $\quad 1 \leq i \leq n$.

Sejam $\Phi, \Phi_{1}$ e $\Phi_{2}$ as funções definidas em I e com valores em $\mathbb{R}$ dadas por

$$
\begin{gathered}
\Phi(s)=\left|\int_{0}^{s} \varphi(t) d t\right| \quad, \quad \Phi_{1}=\left.\psi\right|_{I} \quad \text { e } \\
\Phi_{2}(s)=b s, \text { se } s \in\left[0, \infty\left[\cap I \quad \text { e } \quad \Phi_{2}(s)=a s, \text { se } s \in\right]-\infty, 0[\cap I .\right.
\end{gathered}
$$

Então $\Phi, \Phi_{1}$ e $\Phi_{2} \in \mathcal{C}(I ; \mathbb{R})$ e temos, por (2) e (iii), que

$$
\left.\Phi_{1}(s) \leq \frac{\partial \hat{g}_{i}}{\partial y}(\varepsilon, s, y) \leq \Phi_{2}(s), \quad \text { para todo }(\varepsilon, s, y) \in\right] 0, \tau[\times I \times \mathbb{R} \text { e } 1 \leq i \leq n .
$$


Como $J \subset \psi^{-1}(]-1, \infty[) \cap I=\Phi_{1}^{-1}(]-1, \infty[)$ e valem (1) e (3) temos por 1.2.24 que, se $\widehat{Y}$ é a aplicação moderada definida em $] 0,1] \times I \times \mathbb{R}^{n}$ por

$$
\hat{Y}\left(\varepsilon, s, r_{1}, \ldots, r_{n}\right)=\left(s, \widehat{g}_{1}\left(\varepsilon, s, r_{1}\right)+r_{1}, \ldots, \widehat{g}_{n}\left(\varepsilon, s, r_{n}\right)+r_{n}\right)=\left(s, \widehat{X}\left(\varepsilon, s, r_{1}, \ldots, r_{n}\right)\right)
$$

e $Y$ é a classe de $\widehat{Y}$ em $\mathcal{G}\left(I \times \mathbb{R}^{n} ; \mathbb{R}^{n+1}\right)$, então

$$
\begin{aligned}
& \left.Y\right|_{J \times R^{n}} \in \mathcal{G}_{*}\left(J \times \mathbb{R}^{n} ; J \times \mathbb{R}^{n}\right) ; \\
& \left.Y\right|_{J \times R^{n}} \text { é uma aplicação inversível; }
\end{aligned}
$$

se $L \subset \subset J$ e $K \subset \subset \mathbb{R}^{n}$, então $\inf \{J \hat{Y}(\varepsilon, s, r):(\varepsilon, s, r) \in] 0, \tau[\times J \times K\}>0$;

existe um representante $\widehat{\Gamma}=\left(\widehat{\Gamma}_{0}, \widehat{\Gamma}_{1}, \ldots, \widehat{\Gamma}_{n}\right)$ de $\left(\left.Y\right|_{J \times R^{n}}\right)^{-1}$ tal que

$$
\left.\widehat{\Gamma}(\varepsilon, .)=\left(\left.\hat{Y}(\varepsilon, .)\right|_{J \times R^{n}}\right)^{-1} \text {, para todo } \varepsilon \in\right] 0, \tau[\text {. }
$$

Sejam $X$ a classe $\widehat{X}$ e $P$ a classe de $\widehat{P}$ em $\mathcal{G}\left(I \times \mathbb{R}^{n} ; \mathbb{R}^{n}\right)$. Então, por (i) é claro que $\left.P\right|_{J \times R^{n}}$ pertence a $\mathcal{G}_{*}\left(J \times \mathbb{R}^{n} ; \mathbb{R}^{n}\right)$. Provaremos a seguir que $X \in \mathcal{G}_{*}\left(I \times \mathbb{R}^{n} ; \mathbb{R}^{n}\right)$.

Seja $K^{\prime} \subset \subset I \times \mathbb{R}^{n}$ e tomemos $L$ um intervalo fechado de $\mathbb{R}$ e $d>0$ tais que $0 \in L \subset I$ e $K^{\prime} \subset L \times{\overline{I_{d}}}^{n}$.

Usando o Teorema do Valor Médio, (ii) e (iii) temos que, se $\varepsilon \in] 0, \tau[$, então dados $(t, y) \in L \times \bar{I}_{d}$ e $1 \leq i \leq n$, existe $\omega_{i}$ entre $a_{i}$ e $y$ tal que

$$
\begin{aligned}
\left|\frac{\partial \hat{h}_{i}}{\partial y}\left(\varepsilon, t, \frac{\partial \widehat{f}_{i}}{\partial y}(\varepsilon, y)\right)\right| & =\left|\frac{\partial \widehat{h}_{i}}{\partial y}\left(\varepsilon, t, \frac{\partial \widehat{f}_{i}}{\partial y}\left(\varepsilon, a_{i}\right)\right)+\frac{\partial^{2} \widehat{h}_{i}}{\partial y^{2}}\left(\varepsilon, t, \frac{\partial \widehat{f}_{i}}{\partial y}\left(\varepsilon, \omega_{i}\right)\right) \frac{\partial^{2} \widehat{f}_{i}}{\partial y^{2}}\left(\varepsilon, \omega_{i}\right)\left(y-a_{i}\right)\right| \\
& \leq \varphi(t)+\left|y-a_{i}\right| \max \{|a|,|b|\} .
\end{aligned}
$$

$$
\left|\frac{\partial \widehat{h}_{i}}{\partial y}\left(\varepsilon, t, \frac{\partial \widehat{f}_{i}}{\partial y}(\varepsilon, y)\right)\right| \leq \max \{\varphi(s): s \in L\}+\left(d+\left|a_{i}\right|\right) \max \{|a|,|b|\},
$$

para todo $(\varepsilon, t, y) \in] 0, \tau\left[\times L \times \overline{I_{d}}\right.$ e $1 \leq i \leq n$.

Seja $c=\max \{|t|: t \in L\}\left[\max \{\varphi(t): t \in L\}+\left(d+\|\left(a_{1}, \ldots, a_{n}\right)||\right) \max \{|a|,|b|\}\right]$. Então, por (8), temos que

$$
\left.\left|\widehat{g}_{i}(\varepsilon, s, y)\right| \leq c, \quad \text { para todo }(\varepsilon, s, y) \in\right] 0, \tau\left[\times L \times \overline{I_{d}} \quad \text { e } \quad 1 \leq i \leq n\right.
$$

Usando que $K^{\prime} \subset L \times{\overline{I_{d}}}^{n}$ e (9) concluímos que

$$
\left\{\widehat{X}\left(\varepsilon, s, r_{1}, \ldots, r_{n}\right):\left(\varepsilon, s, r_{1}, \ldots, r_{n}\right) \in\right] 0, \tau\left[\times K^{\prime}\right\} \subset \overline{B_{n(c+d)}(0)} \subset \subset \mathbb{R}^{n}
$$


Portanto $X \in \mathcal{G}_{*}\left(I \times \mathbb{R}^{n} ; \mathbb{R}^{n}\right)$, e assim $\left.X\right|_{J \times R^{n}} \in \mathcal{G}_{*}\left(J \times \mathbb{R}^{n} ; \mathbb{R}^{n}\right)$.

Como $\left.X\right|_{J \times R^{n}}$ e $\left.P\right|_{J \times R^{n}}$ pertencem a $\mathcal{G}_{*}\left(J \times \mathbb{R}^{n} ; \mathbb{R}^{n}\right),\left(\left.X\right|_{J \times R^{n}},\left.P\right|_{J \times R^{n}}\right)$ satisfaz 2.1.1.ii e 2.1.1.iii e valem (4) e (5) temos que

$$
\left(\left.X\right|_{J \times R^{n}},\left.P\right|_{J \times R^{n}}\right) \in \mathcal{S}\left(I, \mathbb{R}^{n}, \mathbb{R}^{n}, H, f, J, J \times \mathbb{R}^{n}\right),
$$

e assim, por 2.1.3 temos que $u=U \circ\left(\left.Y\right|_{J \times R^{n}}\right)^{-1}$ é uma solução para o problema HJ em $\mathcal{G}\left(J \times \mathbb{R}^{n} ; \mathbb{R}\right)$ e $\left(\frac{\partial u}{\partial x_{1}}, \ldots, \frac{\partial u}{\partial x_{n}}\right)=P \circ\left(\left.Y\right|_{J \times R^{n}}\right)^{-1}$, onde $l$ é como em $2.1 .3 \mathrm{e}$ $(t, x)=\left(t, x_{1}, \ldots, x_{n}\right)$ denota um ponto genérico de $J \times \mathbb{R}^{n}$.

Portanto (I) é verdadeira. Provaremos a seguir (II).

Seja $\hat{v}=\hat{P} \circ \widehat{\Gamma}$, sendo $\widehat{\Gamma}$ como em (7). Então $\widehat{v}=\left(\widehat{v}_{1}, \ldots, \widehat{v}_{n}\right)$ é um representante de $\left(\frac{\partial u}{\partial x_{1}}, \ldots, \frac{\partial u}{\partial x_{n}}\right)$.

Suponhamos $\widehat{Y}=\left(\widehat{Y}_{0}, \widehat{Y}_{1}, \ldots, \widehat{Y}_{n}\right)$ e sejam $\Phi_{1}$ e $\Phi_{2}$ definidas anteriormente (antes da afirmação (3)). Então, usando (3), temos que

$$
\begin{aligned}
& \left|\frac{\partial \widehat{Y}_{0}}{\partial s}\left(\varepsilon, s, r_{1}, \ldots, r_{n}\right)\right|=1 ; \quad ; \frac{\partial \widehat{Y}_{0}}{\partial r_{j}}\left(\varepsilon, s, r_{1}, \ldots, r_{n}\right) \mid=0 ; \\
& \left|\frac{\partial \widehat{Y}_{i}}{\partial s}\left(\varepsilon, s, r_{1}, \ldots, r_{n}\right)\right|=\left|\frac{\partial \widehat{g}_{i}}{\partial s}\left(\varepsilon, s, r_{i}\right)\right|=\left|\frac{\partial \hat{h}_{i}}{\partial y}\left(\varepsilon, s, \frac{\partial \widehat{f}_{i}}{\partial y}\left(\varepsilon, r_{i}\right)\right)\right| ; \\
& \left|\frac{\partial \widehat{Y}_{i}}{\partial r_{j}}\left(\varepsilon, s, r_{1}, \ldots, r_{n}\right)\right| \leq 1+\left|\frac{\partial \widehat{g}_{i}}{\partial y}\left(\varepsilon, s, r_{i}\right)\right| \leq 1+\max \left\{\left|\Phi_{1}(s)\right|,\left|\Phi_{2}(s)\right|\right\},
\end{aligned}
$$

para todo $\left.\left(\varepsilon, s, r_{1}, \ldots, r_{n}\right) \in\right] 0, \tau\left[\times J \times \mathbb{R}^{n}\right.$.

Portanto, usando que (8) é verdadeira para $L \times \overline{I_{d}}$, sendo $L \subset \subset J$ e $d>0$, concluímos que

$\partial^{\alpha} Y_{i} \in \mathcal{G}_{*}\left(J \times \mathbb{R}^{n} ; \mathbb{R}^{n+1}\right)$, para todo $\alpha \in \mathbb{N}^{n+1}$ com $|\alpha|=1$ e $0 \leq i \leq n$

Notemos que, se $1 \leq i, j \leq n$ e $(\varepsilon, t, x) \in] 0, \tau\left[\times J \times \mathbb{R}^{n}\right.$, então

$$
\frac{\partial \widehat{v}_{i}}{\partial x_{j}}(\varepsilon, t, x)=\frac{\partial}{\partial x_{j}}\left(\widehat{P}_{i} \circ \widehat{\Gamma}\right)(\varepsilon, t, x)=\frac{\partial^{2} \widehat{f}_{i}}{\partial y^{2}}\left(\varepsilon, \widehat{\Gamma}_{i}(\varepsilon, t, x)\right) \frac{\partial \widehat{\Gamma}_{i}}{\partial x_{j}}(\varepsilon, t, x) \text {. }
$$

Usando (i), (6), (10), 1.2.17 e (11) obtemos (II). //

A seguir apresentamos algumas funções para as quais podemos aplicar o resultado anterior. 
2.1.8 Exemplo. Sejam $I$ um intervalo aberto de $\mathbb{R}$ com $0 \in I, \mu, \nu$ e $\tilde{\nu}$ funções definidas em $] 0,1]$ e com valores em $\mathbb{R}$ e $\psi, \Psi$ e $\Phi$ funçôes pertencentes a $\mathcal{C}^{\infty}(\mathbb{R} ; \mathbb{R})$ tais que $\mu, \nu, \tilde{\nu}, \psi, \Psi$ e $\Phi$ sâo funçôes limitadas. Então as funçôes moderadas definidas em $] 0,1] \times \mathbb{R}$ por

$$
\hat{g}(\varepsilon, y)=\int_{0}^{y}\left(\int_{0}^{\lambda} \Psi(\nu(\varepsilon) x) d x\right) d \lambda \quad e \quad \hat{l}(\varepsilon, y)=\nu(\varepsilon) \int_{0}^{y}\left(\int_{0}^{\lambda} \Psi(x) d x\right) d \lambda
$$

podem ser usadas para $\hat{f}_{i}$ em 2.1 .7 e a função modercáa definida em $\left.] 0,1\right] \times I \times \mathbb{R}$ por

$$
\widehat{h}(\varepsilon, t, y)=\psi(\mu(\varepsilon) t) \int_{0}^{y}\left(\int_{0}^{\lambda} \Phi(\tilde{\nu}(\varepsilon) x) d x\right) d \lambda
$$

ou por, se $\bar{I} \subset \subset \mathbb{R}$,

$$
\widehat{h}(\varepsilon, t, y)=t \mu(\varepsilon) \int_{0}^{y}\left(\int_{0}^{\lambda} \Phi(\tilde{\nu}(\varepsilon) x) d x\right) d \lambda
$$

pode ser usada para $\widehat{h}_{i}$ em 2.1.7.

De fato, basta tomar $a_{i}=0, \varphi=0$ e observar que existe $M>0$ tal que

$$
\max \left\{\left|\frac{\partial^{2} \widehat{h}}{\partial y^{2}}(\varepsilon, t, y)\right|,\left|\frac{\partial^{2} \widehat{g}}{\partial y^{2}}(\varepsilon, y)\right|,\left|\frac{\partial^{2} \widehat{l}}{\partial y^{2}}(\varepsilon, y)\right|\right\} \leq M,
$$

para todo $(\varepsilon, t, y) \in] 0,1] \times I \times \mathbb{R}$.

(As funções $g_{1}(x)=1, g_{2}(x)=\operatorname{arctg} x, g_{3}(x)=\exp \left(-x^{2}\right)$ e $g_{4}(x)=\operatorname{sen}\left(x^{2}\right)$ podem, por exemplo, ser usadas para $\psi, \Psi$ ou $\Phi$.)

Utilizaremos 1.2.28 para apresentar, a seguir, um exemplo em que $\Omega^{\prime}=\mathbb{R}_{+}^{* n}$ e $\frac{\partial H}{\partial x}=0$ em $\mathcal{G}\left(I \times \mathbb{R}^{n} \times \mathbb{R}_{+}^{* n} ; \mathbb{R}^{n}\right)$.

2.1.9 Proposição. Sejam $I$ um intervalo aberto de $\mathbb{R}$ com $0 \in I, 1 \leq k \leq n, l_{k} \in$ $\mathcal{G}_{*}\left(\mathbb{R}_{+}^{*} ; \mathbb{R}_{+}^{*}\right), \hat{l}_{k}$ um representante de $l_{k}, h_{k} \in \mathcal{C}^{\infty}\left(I \times \mathbb{R}_{+}^{*} ; \mathbb{R}\right)$ e $\mu_{k}$ uma função definida em ]0,1] e com valores em $\mathbb{R}$ tais que

(i) $\hat{l}_{k}$ satisfaz de 1.2.26.i até 1.2.26.iii (substituindo $\hat{l}$ por $\hat{l}_{k}$ );

(ii) $h_{k}$ satisfaz de 1.2.26.iv até 1.2.26.vi (substituindo $h$ por $h_{k}$ ); 
(iii) $h_{k}(0,)=$.0 ;

(iv) existem números reais $a_{k}$ e $b_{k}$ tais que $\left.\left.\mu_{k}(] 0,1\right]\right) \subset\left[a_{k}, b_{k}\right] \subset \mathbb{R}_{+}^{*}$.

Se $\widehat{H}$ é a função moderada definida em $] 0,1] \times I \times \mathbb{R}^{n} \times \mathbb{R}_{+}^{* n}$ por

$$
\widehat{H}\left(\varepsilon, t, x_{1}, \ldots, x_{n}, p_{1}, \ldots, p_{n}\right)=\sum_{k=1}^{n} \int_{1}^{p_{k}} \frac{\partial h_{k}}{\partial t}\left(t, \mu_{k}(\varepsilon) y\right) d y,
$$

$H$ a slasse da função $\widehat{H}$ em $\mathcal{G}\left(I \times \mathbb{R}^{n} \times \mathbb{R}_{+}^{* n} ; \mathbb{R}\right), \hat{f}$ a função moderada definida ern ] $0,1] \times \mathbb{R}_{+}^{* n}$ por

$$
\widehat{f}\left(\varepsilon, r_{1}, \ldots, r_{n}\right)=\sum_{k=1}^{n} \int_{1}^{r_{k}} \hat{l}_{k}(\varepsilon, y) d y
$$

e f a classe de $\hat{f}$ em $\mathcal{G}\left(\mathbb{R}_{+}^{* n} ; \mathbb{R}\right)$. Então $\mathcal{S}\left(I, \mathbb{R}^{n}, \mathbb{R}_{+}^{* n}, H, f, I, I \times \mathbb{R}_{+}^{* n}\right) \neq \emptyset$, e em conseqüência existe $u \in \mathcal{G}\left(I \times \mathbb{R}_{+}^{* n} ; \mathbb{R}\right)$ tal que

(I) u é uma solução para o problema $\mathbf{H J}$ em $\mathcal{G}\left(I \times \mathbb{R}_{+}^{* n} ; \mathbb{R}\right)$.

Denotando por $(t, x)=\left(t, x_{1}, \ldots, x_{n}\right)$ um ponto genérico de $\mathbb{R} \times \mathbb{R}_{+}^{* n}$ tem-se que

(II) se $l_{k}^{\prime} \in \mathcal{G}_{*}\left(\mathbb{R}_{+}^{*} ; \mathbb{R}\right)$ para todo $1 \leq k \leq n$, então $\frac{\partial^{2} u}{\partial x_{j} \partial x_{i}} \in \mathcal{G}_{*}\left(I \times \mathbb{R}_{+}^{* n} ; \mathbb{R}\right)$, para todo $1 \leq i, j \leq n$.

Demonstração. Provaremos, em primeiro lugar, que $\mathcal{S}\left(I, \mathbb{R}^{n}, \mathbb{R}_{+}^{* n}, H, f, I, I \times \mathbb{R}_{+}^{* n}\right) \neq$ $\emptyset$ e com o auxílio de 2.1.3 obteremos (I).

Fixemos $1 \leq k \leq n$.

Usando que $\widehat{l}_{k}$ satisfaz 1.2.26.i e (iv) podemos definir a aplicação $\widehat{X}_{k}$ em ]0,1] $\times I \times$ $\mathbb{R}_{+}^{* n}$ por

$$
\widehat{X}_{k}\left(\varepsilon, s, r_{1}, \ldots, r_{n}\right)=r_{k}+h_{k}\left(s, \mu_{k}(\varepsilon) \widehat{l}_{k}\left(\varepsilon, r_{k}\right)\right) .
$$

Seja $\hat{P}_{k}$ a aplicação moderada definida em $\left.] 0,1\right] \times I \times \mathbb{R}_{+}^{* n}$ por

$$
\widehat{P}_{k}\left(\varepsilon, s, r_{1}, \ldots, r_{n}\right)=\widehat{l}_{k}\left(\varepsilon, r_{k}\right) .
$$

Usando que $l_{k} \in \mathcal{G}_{*}\left(\mathbb{R}_{+}^{*} ; \mathbb{R}_{+}^{*}\right), h_{k} \in \mathcal{C}^{\infty}\left(I \times \mathbb{R}_{+}^{*} ; \mathbb{R}\right)$ e (iv) é fácil verificar que $\widehat{X}_{k} \in \mathcal{E}_{M}\left[I \times \mathbb{R}_{+}^{* n} ; \mathbb{R}\right] ;$ 
se $X_{k}$ é a classe de $\widehat{X}_{k}$ em $\mathcal{G}\left(I \times \mathbb{R}_{+}^{* n} ; \mathbb{R}\right)$, então $X_{k} \in \mathcal{G}_{*}\left(I \times \mathbb{R}_{+}^{* n} ; \mathbb{R}\right)$;

se $P_{k}$ é a classe de $\hat{P}_{k}$ em $\mathcal{G}\left(I \times \mathbb{R}_{+}^{* n} ; \mathbb{R}\right)$, então $P_{k} \in \mathcal{G}_{*}\left(I \times \mathbb{R}_{+}^{* n} ; \mathbb{R}_{+}^{*}\right)$;

se $\widehat{\psi}_{k}$ é a função moderada definida em $\left.] 0,1\right] \times \mathbb{R}_{+}^{*}$ por

$$
\widehat{\psi}_{k}(\varepsilon, y)=\mu_{k}(\varepsilon) \widehat{l}_{k}(\varepsilon, y)
$$

e se $\psi_{k}$ é a classe de $\widehat{\psi}_{k}$ em $\mathcal{G}\left(\mathbb{R}_{+}^{*} ; \mathbb{R}\right)$, então

$$
\left.\left.\widehat{\psi}_{k}(] 0,1\right] \times \mathbb{R}_{+}^{*}\right) \subset \mathbb{R}_{+}^{*} \quad \text { e } \quad \psi_{k} \in \mathcal{G}_{*}\left(\mathbb{R}_{+}^{*} ; \mathbb{R}_{+}^{*}\right)
$$

Sejam $\widehat{X}=\left(\widehat{X}_{1}, \ldots, \widehat{X}_{n}\right)$ e $\widehat{P}=\left(\widehat{P}_{1}, \ldots, \widehat{P}_{n}\right), \widehat{Y}=(\widehat{\pi}, \widehat{X}), X$ a classe de $\widehat{X}$ e $P$ a classe de $\widehat{P}$ em $\mathcal{G}\left(I \times \mathbb{R}_{+}^{* n} ; \mathbb{R}^{n}\right)$. Então

$$
X \in \mathcal{G}_{*}\left(I \times \mathbb{R}_{+}^{* n} ; \mathbb{R}^{n}\right) \quad \text { e } \quad P \in \mathcal{G}_{*}\left(I \times \mathbb{R}_{+}^{* n} ; \mathbb{R}_{+}^{* n}\right) .
$$

Usando (i) e (iv) concluímos, para todo $1 \leq k \leq n$, que

$$
\left.\left.\widehat{\psi}_{k}^{\prime}(\varepsilon, y) \geq 0 \quad \text { para todo }(\varepsilon, y) \in\right] 0,1\right] \times \mathbb{R}_{+}^{*}
$$

existe $g_{k} \in \mathcal{C}\left(\mathbb{R}_{+}^{*} ; \mathbb{I} i\right)$ função estritamente crescente e tal que

$$
\left.\left.\lim _{x \downarrow 0} g_{k}(x)=0 \text { e } 0<\mu_{k}(\varepsilon) \widehat{l}_{k}(\varepsilon, y) \leq b_{k} \widehat{l}(\varepsilon, y) \leq b_{k} g_{k}(y), \text { para todo }(\varepsilon, y) \in\right] 0,1\right] \times \mathbb{R}_{+}^{*}
$$

e assim se $\varphi_{k}=b_{k} g_{k}$ temos que

$$
\varphi_{k} \in \mathcal{C}\left(\mathbb{R}_{+}^{*} ; \mathbb{R}\right) \text { e } \varphi_{k} \text { é uma função estritamente crescente; }
$$

$$
\left.\left.\lim _{x \downarrow 0} \varphi_{k}(x)=0 \quad \text { e } \quad 0<\widehat{\psi}_{k}(\varepsilon, y) \leq \varphi_{k}(y) \text {, para todo }(\varepsilon, y) \in\right] 0,1\right] \times \mathbb{R}_{+}^{*} .
$$

Como

$\widehat{Y}\left(\varepsilon, s, r_{1}, \ldots, r_{n}\right)=\left(s, \widehat{X}\left(\varepsilon, s, r_{1}, \ldots, r_{n}\right)\right)=\left(s, h_{1}\left(s, \widehat{\psi}_{1}\left(\varepsilon, r_{1}\right)\right)+r_{1}, \ldots, h_{n}\left(s, \widehat{\psi}_{n}\left(\varepsilon, r_{n}\right)\right)+r_{n}\right)$, para todo $\left.\left.\left(\varepsilon, s, r_{1}, \ldots, r_{n}\right) \in\right] 0,1\right] \times I \times \mathbb{R}_{+}^{* n}$, temos, por (1), (3), (4), (5), (ii) e 1.2 .28 , que

$$
\begin{gathered}
Y \in \mathcal{G}_{*}\left(I \times \mathbb{R}_{+}^{* n} ; I \times \mathbb{R}_{+}^{* n}\right) ; \\
Y \text { é uma aplicação inversível; } \\
\left.\left.\inf \left\{J \hat{Y}\left(\varepsilon, s, r_{1}, \ldots, r_{n}\right):\left(\varepsilon, s, r_{1}, \ldots, r_{n}\right) \in\right] 0,1\right] \times I \times \mathbb{R}_{+}^{* n}\right\} \geq 1 ;
\end{gathered}
$$


existe um representante $\widehat{\Gamma}=\left(\widehat{\Gamma}_{0}, \widehat{\Gamma}_{1}, \ldots, \widehat{\Gamma}_{n}\right)$ de $Y^{-1}$ tal que

$$
\left.\left.\widehat{\Gamma}(\varepsilon, .)=(\hat{Y}(\varepsilon, .))^{-1} \text {, para todo } \varepsilon \in\right] 0,1\right] \text {. }
$$

Seja $\Gamma$ a classe de $\hat{\Gamma}$ em $\mathcal{G}\left(I \times \mathbb{R}_{+}^{* n} ; \mathbb{R}^{n+1}\right)$. Então, por (7), temos que $\Gamma \in \mathcal{G}_{*}(I \times$ $\left.\mathbb{R}_{+}^{* n} ; I \times \mathbb{R}_{+}^{* n}\right)$.

Usando as definições de $\widehat{X}$ e $\widehat{P}$ e (iii) é fácil verificar que $(X, P)$ satisfaz 2.1.1.ii e 2.1.1.iii, e assim ut:ilizando (2), (6) e (7) concluímos que

$$
(X, P) \in \mathcal{S}\left(I, \mathbb{R}^{n}, \mathbb{R}_{+}^{* n}, H, f, I, I \times \mathbb{R}_{+}^{* n}\right)
$$

e assim, por 2.1.3, temos que

$$
\begin{aligned}
& u=U \circ Y^{-1} \text { é uma solução para o problema } \mathbf{H J} \text { em } \mathcal{G}\left(I \times \mathbb{R}_{+}^{* n} ; \mathbb{R}\right) ; \\
& \left(\frac{\partial u}{\partial x_{1}}, \ldots, \frac{\partial u}{\partial x_{n}}\right)=P \circ Y^{-1},
\end{aligned}
$$

onde $U$ é como em 2.1.3 e $(t, x)$ denota um ponto genérico de $\mathbb{R} \times \mathbb{R}_{+}^{* n}$, e portanto (I) é verdadeira.

Provaremos a seguir (II).

Seja $\widehat{v}=\hat{P} \circ \hat{\Gamma}$, sendo $\widehat{\Gamma}$ como em (9). Então $\widehat{v}$ é um representante de $\left(\frac{\partial u}{\partial x_{1}}, \ldots, \frac{\partial u}{\partial x_{n}}\right)$. Suponhamos $\hat{Y}=\left(\widehat{Y}_{0}, \widehat{Y}_{1}, \ldots, \widehat{Y}_{n}\right)$. Então

$$
\begin{aligned}
& \left|\frac{\partial \widehat{Y}_{0}}{\partial s}\left(\varepsilon, s, r_{1}, \ldots, r_{n}\right)\right|=1 \quad ; \quad\left|\frac{\partial \widehat{Y}_{0}}{\partial r_{j}}\left(\varepsilon, s, r_{1}, \ldots, r_{n}\right)\right|=0 \\
& \left|\frac{\partial \widehat{Y}_{i}}{\partial s}\left(\varepsilon, s, r_{1}, \ldots, r_{n}\right)\right|=\left|\frac{\partial h_{i}}{\partial t}\left(s, \widehat{\psi}_{i}\left(\varepsilon, r_{i}\right)\right)\right| \\
& \left|\frac{\partial \widehat{Y}_{i}}{\partial r_{j}}\left(\varepsilon, s, r_{1}, \ldots, r_{n}\right)\right| \leq 1+\left|\frac{\partial h_{i}}{\partial y}\left(s, \widehat{\psi}_{i}\left(\varepsilon, r_{i}\right)\right) \mu_{i}(\varepsilon) \widehat{l}_{i}^{\prime}\left(\varepsilon, r_{i}\right)\right|
\end{aligned}
$$

para todo $\left.\left.\left(\varepsilon, s, r_{1}, \ldots, r_{n}\right) \in\right] 0,1\right] \times I \times \mathbb{R}_{+}^{* n}$ e $1 \leq i, j \leq n$.

Portanto, usando (1), (iv) e que $l_{k}^{\prime} \in \mathcal{G}_{*}\left(\mathbb{R}_{+}^{*} ; \mathbb{R}\right)$ e $h_{k} \in \mathcal{C}^{\infty}\left(I \times \mathbb{R}_{+}^{*} ; \mathbb{R}\right)$, para todo $1 \leq k \leq n$, temos que

$$
\partial^{\alpha} Y_{i} \in \mathcal{G}_{*}\left(I \times \mathbb{R}_{+}^{* n} ; \mathbb{R}\right) \text {, para todo } \alpha \in \mathbb{N}^{n+1} \text { com }|\alpha|=1 \text { e } 0 \leq i \leq n,
$$

e assim, por (8), (10) e 1.2.17, concluímos que se $\Gamma=\left(\Gamma_{0}, \ldots, \Gamma_{n}\right)=Y^{-1}$, então 
$\partial^{\gamma} \Gamma_{i} \in \mathcal{G}_{*}\left(I \times \mathbb{R}_{+}^{* n} ; \mathbb{R}\right)$, para todo $\gamma \in \mathbb{N}^{n+1}$ com $|\gamma|=1$ e $0 \leq i \leq n$.

Notemos que

$$
\left|\frac{\partial \widehat{v}}{\partial x_{i}}(\varepsilon, t, x)\right|=\left|\frac{\partial}{\partial x_{i}}\left(\hat{P}_{i} \circ \widehat{\Gamma}\right)(\varepsilon, t, x)\right|=\widehat{l}_{i}^{\prime}\left(\varepsilon, \widehat{\Gamma}_{i}(\varepsilon, t, x)\right) \frac{\partial \widehat{\Gamma}_{i}}{\partial x_{i}}(\varepsilon, t, x),
$$

para todo $(\varepsilon, t, x) \in] 0,1] \times I \times \mathbb{R}_{+}^{* n}$ e $1 \leq i \leq n$.

Usando (11), (12) e que $\Gamma \in \mathcal{G}_{*}\left(I \times \mathbb{R}_{+}^{* n} ; I \times \mathbb{R}_{+}^{* n}\right)$ e $l_{i}^{\prime} \in \mathcal{G}_{*}\left(\mathbb{R}_{+}^{*} ; \mathbb{R}\right)$, para todo $1 \leq i \leq n$, obtemos (II). //

2.1.10 Observação. As funções $\hat{l}_{i}$ e $\hat{h}_{i}$ apresentadas em 1.2 .27 satisfazem as hipóteses de 2.1.9 (inclusive a hipótese da asserção 2.1.9.II . )

Para finalizar este capítulo utilizaremos 1.2.31 para apresentar um exemplo em que $\left.\Omega^{\prime}=\right] 0,1\left[{ }^{n}\right.$ e $\frac{\partial H}{\partial x}=0$ em $\mathcal{G}\left(I \times \mathbb{R}^{n} \times\right] 0,1\left[{ }^{n} ; \mathbb{R}^{n}\right)$.

2.1.11 Proposição. Sejam $I$ um intervalo aberto de $\mathbb{R}$ com $0 \in I, 1 \leq k \leq n, l_{k} \in$ $\mathcal{G}_{*}(] 0,1[;] 0,1[), \hat{l}_{k}$ um representante de $l_{k}$ e $h_{k} \in \mathcal{C}^{\infty}(] 0,1[; \mathbb{R}) \cap \mathcal{C}([0,1] ; \mathbb{R})$ tais que

(i) $\hat{l}_{k}$ satisfaz de 1.2.29.i até 1.2.29.iii (substituindo $\hat{l}$ por $\hat{l}_{k}$ );

(ii) $h_{k}$ satisfaz 1.2.29.iv (substituindo $h$ por $h_{k}$ ).

Se $W=\left\{\left(t, y_{1}, \ldots, y_{n}\right) \in I \times \mathbb{R}^{n}: 0<y_{k}<1+t^{2} h_{k}(1)\right.$, para todo $\left.1 \leq k \leq n\right\}$, $V=\left\{y \in \mathbb{R}^{n}:(0, y) \in W\right\}, \widehat{H}$ é a função moderada definida em $\left.\left.] 0,1\right] \times I \times \mathbb{R}^{n} \times\right] 0,1\left[{ }^{n}\right.$ por

$$
\widehat{H}\left(\varepsilon, t, x_{1}, \ldots, x_{n}, p_{1}, \ldots, p_{n}\right)=2 t \sum_{k=1}^{n} \int_{\frac{1}{2}}^{p_{k}} h_{k}(y) d y,
$$

$H$ a classe da função $\widehat{H}$ em $\mathcal{G}\left(I \times \mathbb{R}^{n} \times\right] 0,1\left[{ }^{n} ; \mathbb{R}\right), \hat{f}$ a função moderada definida em ] $0,1] \times] 0,1\left[{ }^{n}\right.$ por

$$
\widehat{f}\left(\varepsilon, r_{1}, \ldots, r_{n}\right)=\sum_{k=1}^{n} \int_{\frac{1}{2}}^{r_{k}} \widehat{l}_{k}(\varepsilon, y) d y
$$

e $f$ a classe de $\hat{f}$ em $\mathcal{G}(] 0,1\left[{ }^{n} ; \mathbb{R}\right)$. Então $\mathcal{S}\left(I, \mathbb{R}^{n},\right] 0,1\left[{ }^{n}, H, f, I, W\right) \neq \emptyset$, e em conseqüência existe $u \in \mathcal{G}(W ; \mathbb{R})$ tal que 
(I) u é uma solução para o probema $\mathbf{H J}$ em $\mathcal{G}(W ; \mathbb{R})$.

Denotando por $(t, x)=\left(t, x_{1}, \ldots, x_{n}\right)$ um ponto genérico de $\mathbb{R}^{n+1}$ tem-se que

(II) se $l_{k}^{\prime} \in \mathcal{G}_{*}(] 0,1[; \mathbb{R})$ para todo $1 \leq k \leq n$, então $\frac{\partial^{2} u}{\partial x_{j} \partial x_{i}} \in \mathcal{G}_{*}(W ; \mathbb{R})$, para todo $1 \leq i, j \leq n$.

Demonstração. Provaremos, em primeiro lugar, que

$$
\mathcal{S}\left(I, \mathbb{R}^{n},\right] 0,1\left[{ }^{n}, H, f, I, W\right) \neq \emptyset
$$

e com o auxílio de 2.1 .3 obteremos (I).

Fixemos $1 \leq k \leq n$.

Usando que $\widehat{l}_{k}$ satisfaz 1.2.29.i podemos definir a aplicação $\widehat{X}_{k}$ em $\left.\left.] 0,1\right] \times I \times\right] 0,1{ }^{n}$ por

$$
\widehat{X}_{k}\left(\varepsilon, s, r_{1}, \ldots, r_{n}\right)=r_{k}+s^{2} h_{k}\left(\widehat{l}_{k}\left(\varepsilon, r_{k}\right)\right)
$$

Seja $\widehat{P}_{k}$ a aplicação moderada definida em $\left.\left.] 0,1\right] \times I \times\right] 0,1\left[{ }^{n}\right.$ por

$$
\widehat{P}_{k}\left(\varepsilon, s, r_{1}, \ldots, r_{n}\right)=\widehat{l}_{k}\left(\varepsilon, r_{k}\right)
$$

Usando que $l_{k} \in \mathcal{G}_{*}(] 0,1[;] 0,1[)$ e $h_{k} \in \mathcal{C}^{\infty}(] 0,1[; \mathbb{R})$ é fácil verificar que $\widehat{X}_{k} \in \mathcal{E}_{M}[I \times] 0,1\left[{ }^{n} ; \mathbb{R}\right]$

se $X_{k}$ é a classe de $\widehat{X}_{k}$ em $\mathcal{G}(I \times] 0,1\left[{ }^{n} ; \mathbb{R}\right)$, então $X_{k} \in \mathcal{G}_{*}(I \times] 0,1\left[{ }^{n} ; \mathbb{R}\right)$;

se $P_{k}$ é a classe de $\widehat{P}_{k}$ em $\mathcal{G}(I \times] 0,1\left[{ }^{n} ; \mathbb{R}\right)$, então $P_{k} \in \mathcal{G}_{*}(I \times] 0,1\left[{ }^{n} ;\right] 0,1[)$;

Sejam $\widehat{X}=\left(\widehat{X}_{1}, \ldots, \widehat{X}_{n}\right), \widehat{P}=\left(\widehat{P}_{1}, \ldots, \widehat{P}_{n}\right), \widehat{Y}=(\widehat{\pi}, \widehat{X}), X$ a classe de $\widehat{X}$ e $P$ a clase de $\widehat{P}$ em $\mathcal{G}(I \times] 0,1\left[{ }^{n} ; \mathbb{R}^{n}\right)$ e $Y$ a classe de $\hat{Y}$ em $\mathcal{G}(I \times] 0,1\left[{ }^{n} ; \mathbb{R}^{n+1}\right)$. Então

$$
X \in \mathcal{G}_{*}(I \times] 0,1\left[{ }^{n} ; \mathbb{R}^{n}\right) \quad \text { e } \quad P \in \mathcal{G}_{*}(I \times] 0,1\left[{ }^{n} ;\right] 0,1\left[{ }^{n}\right) .
$$

Como

$\hat{Y}\left(\varepsilon, s, r_{1}, \ldots, r_{n}\right)=\left(s, \widehat{X}\left(\varepsilon, s, r_{1}, \ldots, r_{n}\right)\right)=\left(s, s^{2} h_{1}\left(\widehat{l}_{1}\left(\varepsilon, r_{1}\right)\right)+r_{1}, \ldots, s^{2} h_{n}\left(\widehat{l}_{n}\left(\varepsilon, r_{n}\right)\right)+r_{n}\right)$ 
para todo $\left.\left.\left.\left(\varepsilon, s, r_{1}, \ldots, r_{n}\right) \in\right] 0,1\right] \times I \times\right] 0,1\left[{ }^{n}\right.$, temos, por (i), (ii) e 1.2 .31 , que

$$
\begin{aligned}
& Y \in \mathcal{G}_{*}(I \times] 0,1\left[{ }^{n} ; W\right) \\
& Y \text { é uma aplicação inversível; }
\end{aligned}
$$

$$
\left.\left.\inf \left\{J \widehat{Y}\left(\varepsilon, s, r_{1}, \ldots, r_{n}\right):\left(\varepsilon, s, r_{1}, \ldots, r_{n}\right) \in\right] 0,1\right] \times I \times\right] 0,1\left[{ }^{n}\right\} \geq 1
$$

existe um representante $\widehat{\Gamma}=\left(\widehat{\Gamma}_{0}, \widehat{\Gamma}_{1}, \ldots, \widehat{\Gamma}_{n}\right)$ de $Y^{-1}$ tal que

$$
\left.\left.\widehat{\Gamma}(\varepsilon, .)=(\widehat{Y}(\varepsilon, .))^{-1} \text {, para todo } \varepsilon \in\right] 0,1\right] \text {. }
$$

Seja $\Gamma$ a classe de $\widehat{\Gamma}$ em $\mathcal{G}\left(W ; \mathbb{R}^{n+1}\right)$. Então, por $(3)$, temos que $\Gamma \in \mathcal{G}_{*}(W ; I \times] 0,1\left[{ }^{n}\right)$. Usando as definições de $\widehat{X}$ e $\widehat{P}$ é fácil verificar que $(X, P)$ satisfaz 2.1.1.ii e 2.1.1.iii, e assim utilizando (1), (2) e (3) concluímos que

$$
(X, P) \in \mathcal{S}\left(I, \mathbb{R}^{n},\right] 0,1\left[{ }^{n}, H, f, I, W\right)
$$

e assim, por 2.1.3 temos que $u=U \circ Y^{-1}$ é uma solução para o probleme $\mathbf{H J}$ em $\mathcal{G}(W ; \mathbb{R})$ e $\left(\frac{\partial u}{\partial x_{1}}, \ldots, \frac{\partial u}{\partial x_{n}}\right)=P \circ Y^{-1}$, onde $U$ é como em 2.1 .3 e $(t, x)$ denota um ponto genérico de $\mathbb{R}^{n+1}$, e portanto (I) é verdadeira.

Provaremos a seguir (II).

Seja $\widehat{v}=\widehat{P} \circ \widehat{\Gamma}$, sendo $\widehat{\Gamma}$ como em (5). Então $\widehat{v}$ é um representante de $\left(\frac{\partial u}{\partial x_{1}}, \ldots, \frac{\partial u}{\partial x_{n}}\right)$.

Suponhamos $\hat{Y}=\left(\widehat{Y}_{0}, \widehat{Y}_{1}, \ldots, \widehat{Y}_{n}\right)$. Então

$$
\begin{aligned}
& \left|\frac{\partial \widehat{Y}_{0}}{\partial s}\left(\varepsilon, s, r_{1}, \ldots, r_{n}\right)\right|=1 ; \quad\left|\frac{\partial \widehat{Y}_{0}}{\partial r_{j}}\left(\varepsilon, s, r_{1}, \ldots, r_{n}\right)\right|=0 ; \\
& \left|\frac{\partial \widehat{Y}_{i}}{\partial s}\left(\varepsilon, s, r_{1}, \ldots, r_{n}\right)\right|=\left|2 s h_{k}\left(\widehat{l}_{k}\left(\varepsilon, r_{k}\right)\right)\right| ; \\
& \left|\frac{\partial \widehat{Y}_{i}}{\partial r_{j}}\left(\varepsilon, s, r_{1}, \ldots, r_{n}\right)\right| \leq 1+s^{2}\left|h_{k}^{\prime}\left(l_{k}\left(\varepsilon, r_{k}\right)\right) \widehat{l}_{k}^{\prime}\left(\varepsilon, r_{k}\right)\right|,
\end{aligned}
$$

para todo $\left.\left.\left.\left(\varepsilon, s, r_{1}, \ldots, r_{n}\right) \in\right] 0,1\right] \times I \times\right] 0,1\left[{ }^{n} \quad\right.$ e $1 \leq i, j \leq n$.

Portanto, usando que $l_{k}^{\prime} \in \mathcal{G}_{*}(] 0,1[; \mathbb{R})$ e $h_{k} \in \mathcal{C}^{\infty}(] 0,1[; \mathbb{R})$ para todo $1 \leq k \leq n$, temos que

$$
\partial^{\alpha} Y_{i} \in \mathcal{G}_{*}(I \times] 0,1\left[{ }^{n} ; \mathbb{R}\right) \text {, para todo } \alpha \in \mathbb{N}^{n+1} \text { com }|\alpha|=1 \text { e } 0 \leq i \leq n,
$$


e assim, por (4), (6) e 1.2.17, concluímos que

$\partial^{\gamma} \Gamma_{i} \in \mathcal{G}_{*}(W ; \mathbb{R})$, para todo $\gamma \in \mathbb{N}^{n+1}$ com $|\gamma|=1$ e $0 \leq i \leq n$.

Notemos ainda que

$$
\frac{\partial \widehat{v}}{\partial x_{i}}(\varepsilon, t, x)=\frac{\partial}{\partial x_{i}}\left(\hat{P}_{i} \circ \Gamma\right)(\varepsilon, t, x)=\widehat{l}_{i}^{\prime}\left(\varepsilon, \widehat{\Gamma}_{i}(\varepsilon, t, x)\right) \frac{\partial \Gamma_{i}}{\partial x_{i}}(\varepsilon, t, x),
$$

para todo $(\varepsilon, t, x) \in] 0,1] \times W$.

Usando (7), (8), que $\Gamma \in \mathcal{G}_{*}(W ; \mathbb{R} \times] 0,1\left[{ }^{n}\right)$ e que $l_{i}^{\prime} \in \mathcal{G}_{*}(] 0,1[; \mathbb{R})$, para todo $1 \leq i \leq n$, obtemos (II). //

2.1.12 Observação. As funções $\hat{l}_{i}$ e $\widehat{h}_{i}$ apresentadas em 1.2.30 satisfazem as hipóteses de 2.1.11.

Para apresentar exemplos de funções $H$ e $f$, para as quais $\mathcal{S}\left(I, \Omega, \Omega^{\prime}, H, f, J, W\right) \neq \emptyset$ (e portanto o problema HJ tem solução), sem a condição $\frac{\partial H}{\partial x}=0$ (ver 3.3.1) precisamos saber um pouco mais sobre equações diferenciais ordinárias no contexto das funções generalizadas. É isto que faremos no próximo capítulo. 


\section{Capítulo 3}

\section{Equação de Hamilton-Jacobi : Unicidade de soluções}

Em 2.1.2 construímos uma solução para o problema HJ com o auxílio de uma aplicação generalizada cuja derivada na primeira variável era uma solução de um certo sistema de equações diferenciais ordinárias que envolvia funções generalizadas. O estudo de funções com essa propriedade está na seção 3.2 .

Em 3.3 obteremos, utilizando os resultados apresentados em 3.2, mais exemplos de funções generalizadas $H$ e $f$ para as quais o problema $\mathbf{H J}$ tem solução e estabeleceremos alguns resultados sobre a unicidade de soluções.

$\mathrm{Na}$ seção 3.1 apresentaremos alguns resultados sobre a existência e unicidade de soluções para um sistema de equações diferenciais ordinárias no contexto das funções generalizadas de Colombeau que, apesar de não ser usado neste trabalho, pensamos ter algum interesse em um estudo introdutório de E.D.O. envolvendo funções generalizadas. Assim a leitura da seção 3.1 não é necessária para a compreensão do restante do capítulo 3 e de sua integração com o capítulo 2 .

Em todo o capítulo utilizaremos a notação apresentada em 3.1.8 e as definições 3.1.1 e 3.1.4. 


\subsection{Equações diferenciais ordinárias : existência e unicidade de soluções}

Quando estudamos, no caso clássico, um problema de E.D.O. com uma condição inicial dada começamos analisando o seguinte problema: Dados $\Omega$ um aberto de $\mathbb{R}^{n}$, I um intervalo aberto de $\mathbb{R}, t_{o} \in I, x_{o} \in \mathbb{R}^{n}$ e $f \in \mathcal{C}\left(I \times \Omega ; \mathbb{R}^{n}\right)$, existem a $>0$ e uma aplicação $u \in \mathcal{C}(] t_{o}-a, t_{o}+a\left[; \mathbb{R}^{n}\right)$ satisfazendo $\left[t_{o}-a, t_{o}+a\right] \subset I e$

$$
u^{\prime}(t)=f(t, u(t)) \quad u\left(t_{0}\right)=x_{0} \quad ?
$$

Em caso afirmativo a aplicação u é única? Existe uma solução de (1) que é uma soluçâo maximal (isto é, o domínio de definição da solução é maximal) ?

Nesta seção analisaremos o problema acima admitindo $f \in \mathcal{G}\left(I \times \Omega ; \mathbb{R}^{n}\right)$ e $x_{\circ}$ um vetor generalizado. Neste caso, as equações que aparecem em (1) serão escritas na forma

$$
u^{\prime}=f \circ\left(1_{t_{o}-a, t_{o}+a[}, u\right) \quad e \quad u\left(t_{o}\right)=x_{\circ},
$$

sendo $u \in \mathcal{G}_{*}(] t_{o}-a, t_{o}+a[; \Omega)$.

Para facilitar a escrita de (2) escreveremos $\pi_{*}$ no lugar de $1_{J}$ (definido antes de 1.2.1), sendo $J$ qualquer intervalo aberto de $\mathbb{R}$. Portanto (2) será escrita na forma

$$
u^{\prime}=f \circ\left(\pi_{*}, u\right) \quad u\left(t_{o}\right)=x_{o} .
$$

Os principais resultados desta seção são $3.1 .9,3.1 .13$ e 3.1 .16 .

3.1.1 Definição. Seja $\Omega$ um aberto de $\mathbb{R}^{n}$. Dizemos que

(i) $\hat{f} \in \mathcal{E}_{M}\left[\Omega ; \mathbb{R}^{p}\right]$ é uma aplicação limitada em $\Omega$ se, e somente se, existem $M>0$ e $\eta \in] 0,1]$ tais que $\|\widehat{f}(\varepsilon, x)\| \leq M$, para todo $(\varepsilon, x) \in] 0, \eta[\times \Omega$;

(ii) $\widehat{f} \in \mathcal{E}_{M}\left[\Omega ; \mathbb{R}^{p}\right]$ tem a propriedade (CLL) (crescimento logarítmico local) em $\Omega$ se, e somente se, dado qualquer $K \subset \subset \Omega$, existem $N \in \mathbb{N}, c>0$ e $\eta \in] 0,1]$ tais que $\|\widehat{f}(\varepsilon, x)\| \leq \ln \left(c \varepsilon^{-N}\right)$, para todo $\left.(\varepsilon, x) \in\right] 0, \eta[\times K$; 
(iii) $f \in \mathcal{G}\left(\Omega ; \mathbb{R}^{p}\right)$ tem a propriedade (CLL) em $\Omega$ se, e somente se, todo representante de $f$ tem a propriedade (CLL) em $\Omega$.

Convém observar que fixados $N \in \mathbb{N}$ e $c>0$ existe $\bar{\eta} \in] 0,1\left[\right.$ tal que $c \varepsilon^{-N}>1$, para todo $\varepsilon \in] 0, \bar{\eta}\left[\right.$ e portanto podemos escrever $\ln \left(c \varepsilon^{-N}\right)$ e ainda temos $\ln \left(c \varepsilon^{-N}\right)>0$, para todo $\varepsilon \in] 0, \bar{\eta}[$.

Denotamos por $\mathcal{E}_{M, b[}\left[\Omega ; \mathbb{R}^{p}\right]$ o subconjunto de $\mathcal{E}_{M}\left[\Omega ; \mathbb{R}^{p}\right]$ forrnado pelas aplicações limitadas em $\Omega$ e por $\mathcal{E}_{M, l o g}\left[\Omega ; \mathbb{R}^{p}\right]$ o subconjunto de $\mathcal{E}_{M}\left[\Omega ; \mathbb{R}^{p}\right]$ formado pelas aplicações que têm a propriedade (CLL) em $\Omega$.

Na seção 3.3 utilizaremos a seguinte proposição:

3.1.2 Proposição. Seja $\Omega$ um aberto de $\mathbb{R}^{n}$. As seguintes asserções são verdadeiras:

(I) $\mathcal{E}_{M, l o g}\left[\Omega ; \mathbb{R}^{p}\right]$ é um $\mathbb{R}$-espaço vetorial;

(II) $\mathcal{E}_{M, b}\left[\Omega ; \mathbb{R}^{p}\right] \subset \mathcal{E}_{M, \log }\left[\Omega ; \mathbb{R}^{p}\right]$;

(III) se $\hat{f} \in \mathcal{E}_{M, l o g}\left[\Omega ; \mathbb{R}^{p}\right]$ e $\hat{g} \in \mathcal{E}_{M, b}\left[\Omega ; \mathbb{R}^{p}\right]$, então $\hat{f} \hat{g} \in \mathcal{E}_{M, l o g}\left[\Omega ; \mathbb{R}^{p}\right]$;

(IV) $\mathcal{N}\left[\Omega ; \mathbb{R}^{p}\right] \subset \mathcal{E}_{M, l o g}\left[\Omega ; \mathbb{R}^{p}\right]$

Demonstração. Basta usar as propriedades da função logarítmica e observar, para (II), que se $a>0$, então $a=\ln \left(\varepsilon^{-0}\right.$ expa) e, para (IV), que existe $\left.\eta \in\right] 0,1[$ tal que $\varepsilon \leq-\ln \varepsilon$ para todo $\varepsilon \in] 0, \eta[. / /$

De 3.1.2 obtemos:

3.1.3 Observação. Sejam $\Omega$ um aberto de $\mathbb{R}^{n}$ e $f \in \mathcal{G}\left(\Omega ; \mathbb{R}^{p}\right)$. Então $f$ tem a propriedade (CLL) em $\Omega$ se, e somente se, existe um representante de $f$ que tem a propriedade (CLL) $e m \Omega$.

O subespaço vetorial de $\mathcal{G}\left(\Omega ; \mathbb{R}^{p}\right)$ formado pelas aplicações que tem a propriedade $(\mathrm{CLL})$ em $\Omega$ será denotado por $\mathcal{G}_{\text {log }}\left(\Omega ; \mathbb{R}^{p}\right)$, generalização natural do que foi feito em [3]. 
Definiremos, a seguir, quando que uma função pertencente a $\mathcal{E}_{M}\left[\Omega ; \mathbb{R}^{p}\right] \cup \mathcal{E}_{M}[I \times$ $\left.\Omega ; \mathbb{R}^{p}\right] \cup \mathcal{E}_{M}\left[I \times \Omega \times \Omega^{\prime} ; \mathbb{R}^{p}\right]$ tem a propriedade (LLL) (localmente logaritmicamente lipschitziana).

3.1.4 Definição. Sejam I um intervalo aberto de $\mathbb{R}, \Omega$ um aberto de $\mathbb{R}^{n}, \Omega^{\prime}$ um aberto de $\mathbb{R}^{m}$ e $\hat{f}=\left(\widehat{f}_{1}, \ldots, \hat{f}_{p}\right)$. Dizemos que

(i) $\hat{f} \in \mathcal{E}_{M}\left[\Omega ; \mathbb{R}^{p}\right]$ tem a propriedade (LLL) em $\Omega$ se, e somente se, dados quaisquer $K \subset \subset \Omega$ e $\alpha \in \mathbb{N}^{n}$, existem $N \in \mathbb{N}, c>0$ e $\left.\left.\eta \in\right] 0,1\right]$ tais que

$$
\begin{aligned}
& \left|\widehat{f}_{i}(\varepsilon, x)-\widehat{f}_{i}(\varepsilon, y)\right| \leq \ln \left(c \varepsilon^{-N}\right)\|x-y\| ; \\
& \left|\partial^{\alpha} \widehat{f}_{i}(\varepsilon, x)-\partial^{\alpha} \widehat{f}_{i}(\varepsilon, y)\right| \leq c \varepsilon^{-N}\|x-y\|,
\end{aligned}
$$

para todo $(\varepsilon, x, y) \in] 0, \eta[\times K \times K$ e $1 \leq i \leq p$;

(ii) $\hat{f} \in \mathcal{E}_{M}\left[I \times \Omega ; \mathbb{R}^{p}\right]$ tem a propriedade (LLL) em $(I, \Omega)$ se, e somente se, dados quaisquer $J \subset \subset I, K \subset \subset \Omega$ e $\alpha \in \mathbb{N}^{n+1}$, existem $N \in \mathbb{N}, c>0$ e $\left.\left.\eta \in\right] 0,1\right]$ tais que

$$
\begin{aligned}
& \left|\hat{f}_{i}(\varepsilon, t, x)-\widehat{f}_{i}(\varepsilon, t, y)\right| \leq \ln \left(c \varepsilon^{-N}\right)|| x-y \| ; \\
& \left|\partial^{\alpha} \hat{f}_{i}(\varepsilon, t, x)-\partial^{\alpha} \hat{f}_{i}(\varepsilon, t, y)\right| \leq c \varepsilon^{-N}\|x-y\|,
\end{aligned}
$$

para todo $(\varepsilon, t, x, y) \in] 0, \eta[\times J \times K \times K$ e $1 \leq i \leq p$;

(iii) $\widehat{f} \in \mathcal{E}_{M}\left[I \times \Omega \times \Omega^{\prime} ; \mathbb{R}^{p}\right]$ tem a propriedade (LLL) em $\left(I, \Omega, \Omega^{\prime}\right)$ se, e somente se, dados quaisquer $J \subset \subset I, K \subset \subset \Omega, K^{\prime} \subset \subset \Omega^{\prime}$ e $\alpha \in \mathbb{N}^{n+m+1}$, existem $N \in \mathbb{N}$, $c>0$ e $\eta \in] 0,1]$ tais que

$$
\begin{aligned}
& \left|\widehat{f}_{i}(\varepsilon, t, x, y)-\widehat{f}_{i}(\varepsilon, t, z, y)\right| \leq \ln \left(c \varepsilon^{-N}\right)\|x-z\| \\
& \left|\partial^{\alpha} \widehat{f}_{i}(\varepsilon, t, x, y)-\partial^{\alpha} \widehat{f}_{i}(\varepsilon, t, z, y)\right| \leq c \varepsilon^{-N}\|x-z\|,
\end{aligned}
$$

para todo $(\varepsilon, t, x, z, y) \in] 0, \eta\left[\times J \times K \times K \times K^{\prime}\right.$ e $1 \leq i \leq p$;

(iv) $f \in \mathcal{G}\left(W ; \mathbb{R}^{p}\right)$, sendo $W=\Omega$ ou $W=I \times \Omega$ ou $W=I \times \Omega \times \Omega^{\prime}$, tem a propriedade (LLL) em $\Omega$ ou em $(I, \Omega)$ ou em $\left(I, \Omega, \Omega^{\prime}\right)$ se, e somente se, todo 
representante de $f$ tem a propriedade (LLL) em $\Omega$ ou em $(I, \Omega)$ ou em $\left(I, \Omega, \Omega^{\prime}\right)$, respectivamente.

Usando a definição acima obtemos:

3.1.5 Proposição. Sejam I um intervalo aberto de $\mathbb{R}, \Omega$ um aberto de $\mathbb{R}^{n}$ e $\Omega^{\prime}$ um aberto de $\mathbb{R}^{m}$. Se $W=\Omega$ e $A=\Omega$ ou $W=I \times \Omega$ e $A=(I, \Omega)$ ou $W=I \times \Omega \times \Omega^{\prime}$ e $A=\left(I, \Omega, \Omega^{\prime}\right)$ tem-se, denotando por

$$
\mathcal{E}_{M, L i p, A}\left[W ; \mathbb{R}^{p}\right]
$$

o subconjunto de $\mathcal{E}_{M}\left[W ; \mathbb{R}^{p}\right]$ formado pelas aplicações que têm a propriedade (LLL) em A, que as seguintes asserções são verdadeiras:

(I) $\mathcal{E}_{M, L i p, A}\left[W ; \mathbb{R}^{p}\right]$ é um $\mathbb{R}$-espaço vetorial;

(II) se $\Omega$ é um aberto convexo de $\mathbb{R}^{n}$, então $\mathcal{N}\left[W ; \mathbb{R}^{p}\right] \subset \mathcal{E}_{M, L i p, A}\left[W ; \mathbb{R}^{p}\right]$.

Demonstração. A asserção (I) é imediata. Provaremos (II) admitindo $W=\Omega$ (os outros casos têm prova análoga).

Denotaremos por $x=\left(x_{1}, \ldots, x_{n}\right)$ um ponto genérico de $\mathbb{R}^{n}$.

Sejam $K \subset \subset \Omega, \alpha \in \mathbb{N}^{n}$ e $K_{1}$ a envoltória convexa de $K$.

Como $\hat{f} \in \mathcal{N}\left[W ; \mathbb{R}^{p}\right]$ e $K_{1} \subset \subset \Omega$ existem $c>0$ e $\left.\left.\eta \in\right] 0,1\right]$ tais que $\left|\frac{\partial\left(\partial^{\alpha} \widehat{f}_{i}\right)}{\partial x_{j}}(\varepsilon, x)\right| \leq c \varepsilon$, para todo $\left.(\varepsilon, x) \in\right] 0, \eta\left[\times K_{1}, 1 \leq i \leq p\right.$ e $1 \leq j \leq n$.

Fixemos $\varepsilon \in] 0, \eta[$ e $1 \leq i \leq p$. Então dados quaisquer $x, y \in K$ existe $z$ no segmento de extremidades $x$ e $y$ tal que

$$
\partial^{\alpha} \widehat{f}_{i}(\varepsilon, x)-\partial^{\alpha} \widehat{f}_{i}(\varepsilon, y)=\sum_{j=1}^{n} \frac{\partial\left(\partial^{\alpha} \widehat{f}_{i}\right)}{\partial x_{j}}(\varepsilon, z)\left(x_{j}-y_{j}\right) .
$$

Portanto, por (1) e usando que $K_{1}$ é convexo, temos que $\left|\partial^{\alpha} \widehat{f}_{i}(\varepsilon, x)-\partial^{\alpha} \widehat{f}_{i}(\varepsilon, y)\right| \leq n c \varepsilon|| x-y \|$, para todo $\left.(\varepsilon, x, y) \in\right] 0, \eta[\times K \times K$ e $1 \leq i \leq p$. 
Sejam $N \in \mathbb{N} \operatorname{com} N>n c$ e $\left.\eta_{1} \in\right] 0, \eta[$ tal que $\varepsilon \leq-\ln \varepsilon$ para todo $\varepsilon \in] 0, \eta_{1}[$. Então

$\left|\partial^{\alpha} \widehat{f}_{i}(\varepsilon, x)-\partial^{\alpha} \widehat{f}_{i}(\varepsilon, y)\right| \leq-n c \ln \varepsilon\|x-y\| \leq-N \ln \varepsilon\|x-y\| \leq \ln \left(\varepsilon^{-N}\right)\|x-y\| \leq \varepsilon^{-N}\|x-y\|$, para todo $(\varepsilon, x, y) \in] 0, \eta_{1}[\times K \times K$ e $1 \leq i \leq p$.

Portanto $\hat{f}$ tem a propriedade (LLL) em $A$. //

Quando $\Omega$ é um aberto convexo obtemos o seguinte resultado:

3.1.6 Proposição. Sejam I um intervalo aberto de $\mathbb{R}, \Omega$ um aberto convexo de $\mathbb{R}^{n} e$ $\Omega^{\prime}$ um aberto de $\mathbb{R}^{m}$. São verdadeiras as seguintes asserções:

(I) $\hat{f} \in \mathcal{E}_{M}\left[\Omega ; \mathbb{R}^{p}\right]$ tem a propriedade (LLL) em $\Omega$ se, e somente se, dado qualquér $K \subset \subset \Omega$, existem $N \in \mathbb{N}, c>0$ e $\eta \in] 0,1]$ tais que

$$
\left|\widehat{f}_{i}(\varepsilon, x)-\widehat{f}_{i}(\varepsilon, y)\right| \leq \ln \left(c \varepsilon^{-N}\right)|| x-y \| ;
$$

para todo $(\varepsilon, x, y) \in] 0, \eta[\times K \times K$ e $1 \leq i \leq p$;

(II) $\widehat{f} \in \mathcal{E}_{M}\left[I \times \Omega ; \mathbb{R}^{p}\right]$ tem a propriedade (LLL) em $(I, \Omega)$ se, e somente se, dados quaisquer $J \subset \subset I$ e $K \subset \subset \Omega$, existem $N \in \mathbb{N}, c>0$ e $\eta \in] 0,1]$ tais que

$$
\left|\widehat{f}_{i}(\varepsilon, t, x)-\widehat{f}_{i}(\varepsilon, t, y)\right| \leq \ln \left(c \varepsilon^{-N}\right)\|x-y\| ;
$$

para todo $(\varepsilon, t, x, y) \in] 0, \eta[\times J \times K \times K$ e $1 \leq i \leq p$;

(III) $\widehat{f} \in \mathcal{E}_{M}\left[I \times \Omega \times \Omega^{\prime} ; \mathbb{R}^{p}\right]$ tem a propriedade (LLL) em $\left(I, \Omega, \Omega^{\prime}\right)$ se, e somente se, dados quaisquer $J \subset \subset I, K \subset \subset \Omega$ e $K^{\prime} \subset \subset \Omega^{\prime}$, existem $N \in \mathbb{N}, c>0$ e $\eta \in] 0,1]$ tais que

$$
\left|\widehat{f}_{i}(\varepsilon, t, x, y)-\widehat{f}_{i}(\varepsilon, t, z, y)\right| \leq \ln \left(c \varepsilon^{-N}\right)|| x-z \| ;
$$

para todo $(\varepsilon, t, x, z, y) \in] 0, \eta\left[\times J \times K \times K \times K^{\prime}\right.$ e $1 \leq i \leq p$.

Demonstração. Análoga à de 3.1 .5 , observando que, como $\widehat{f} \in \mathcal{E}_{M}\left[W ; \mathbb{R}^{p}\right]$, sendo $W$ como em 3.1.5, podemos substituir (1) por: existem $N \in \mathbb{N}, c>0$ e $\eta \in] 0,1]$ tais que $\left|\frac{\partial\left(\partial^{\alpha} \widehat{f}_{i}\right)}{\partial x_{j}}(\varepsilon, x)\right| \leq c \varepsilon^{-N}$ para todo $\left.(\varepsilon, x) \in\right] 0, \eta\left[\times K_{1}, 1 \leq i \leq p\right.$ e $1 \leq j \leq n . / /$ 
De 3.1.5.II obtemos:

3.1.7 Observação. Sejam I um intervalo aberto de $\mathbb{R}, \Omega$ um aberto de $\mathbb{R}^{n}$ e $\Omega^{\prime}$ um aberto de $\mathbb{R}^{m}$. Se $W$ e $A$ são como em 3.1 .5 e se $\Omega$ é convexo, então $f \in \mathcal{G}\left(W ; \mathbb{R}^{p}\right)$ tem a propriedade (LLL) em A se, e somente se, existe um representante de $f$ que tem a propriedade (LLL) em $A$.

3.1.8 Notação. Sejam $t_{0} \in \mathbb{R}$ e $a>0$. Denotaremos por $I_{a}\left(t_{0}\right)$ e $\overline{I_{a}\left(t_{0}\right)}$ os intervalos da reta dados, respectivamente, por $] t_{o}-a, t_{o}+a\left[e\left[t_{0}-a, t_{o}+a\right]\right.$. Quando $t_{o}=0$ faremos como em 1.2.21, isto é, escreveremos $I_{a} e \overline{I_{a}}$ no lugar de $I_{a}(0)$ e $\overline{I_{a}(0)}$, respectivamente.

3.1.9 Teorema(Existência). Sejam $\Omega$ um aberto de $\mathbb{R}^{n}$, I um intervalo aberto de $\mathbb{R}$, $t_{o} \in I$ e $x_{o} \in \overline{\mathbb{R}}^{n}$ tal que existe um representante $\widehat{x}_{o}$ de $x_{o}$ satisfazendo:

(i) existem $K \subset \subset \Omega$ e $\tau \in] 0,1]$ tais que $\widehat{x}_{o}(] 0, \tau[) \subset K$.

Se $f \in \mathcal{G}_{*}\left(I \times \Omega ; \mathbb{R}^{n}\right)$, então existem $a>0$ com $\overline{I_{a}\left(t_{o}\right)} \subset I$ e uma aplicação $u \in$ $\mathcal{G}\left(I_{a}\left(t_{o}\right) ; \mathbb{R}^{n}\right)$ tais que

(I) $u \in \mathcal{G}_{*}\left(I_{a}\left(t_{o}\right) ; \Omega\right)$;

(II) $u^{\prime}=f \circ\left(\pi_{*}, u\right)$ em $\mathcal{G}\left(I_{a}\left(t_{o}\right) ; \mathbb{R}^{n}\right)$;

(III) $u\left(t_{o}\right)=x_{o}$.

Demonstração. Seja $\hat{f}=\left(\hat{f}_{1}, \ldots, \widehat{f}_{n}\right)$ um representante de $f$ e sejam $K \subset \subset \Omega$ e $\left.\left.\tau \in\right] 0,1\right]$ como em (i), e tomemos $V$ um aberto de $\Omega \operatorname{com} K \subset V \subset \bar{V} \subset \subset \Omega$ e $a^{*}>0$ tal que $\overline{I_{a^{*}}\left(t_{o}\right)} \subset I$.

Usando que $f \in \mathcal{G}_{*}\left(I \times \Omega ; \mathbb{R}^{n}\right)$ existem $M>0$ e $\left.\eta \in\right] 0, \tau[$ tais que

$$
\|\hat{f}(\varepsilon, t, y)\| \leq M, \text { para todo } \varepsilon \in] 0, \eta\left[\mathrm{e}(t, y) \in \overline{I_{a^{*}}\left(t_{o}\right)} \times \bar{V}\right. \text {. }
$$

Sejam $b$ a distância de $K$ a $\Omega \backslash V$ e $a>0 \operatorname{com} a<\min \left\{a^{*}, \frac{b}{M}\right\}$. 
Fixemos $\varepsilon \in] 0, \eta[$ e seja $T$ a função

$$
T: g \in \mathcal{C}\left(\overline{I_{a}\left(t_{o}\right)} ; \overline{B_{b}\left(\widehat{x}_{o}(\varepsilon)\right)}\right) \longmapsto T g \in \mathcal{C}\left(\overline{I_{a}\left(t_{o}\right)} ; \mathbb{R}^{n}\right)
$$

onde $T g$ é a função definida por

$$
(T g)(t)=\widehat{x}_{o}(\varepsilon)+\int_{t_{o}}^{t} \widehat{f}(\varepsilon, s, g(s)) d s .
$$

Notemos que, se $t \in \overline{I_{a}\left(t_{o}\right)}$, então

$$
\left\|(T g)(t)-\hat{x}_{o}(\varepsilon)\right\| \leq\left|\int_{t_{o}}^{t}\|\hat{f}(\varepsilon, s, g(s))\| d s\right| \leq M\left|t-t_{o}\right| \leq M a<b,
$$

$\mathrm{e} \operatorname{assim}(T g)(t) \in \overline{B_{b}\left(\widehat{x}_{o}(\varepsilon)\right)} \subset \bar{V}$.

Portanto

$$
T: \mathcal{C}\left(\overline{I_{a}\left(t_{o}\right)} ; \overline{B_{b}\left(\widehat{x}_{o}(\varepsilon)\right)}\right) \longmapsto \mathcal{C}\left(\overline{I_{a}\left(t_{o}\right)} ; \overline{B_{b}\left(\widehat{x}_{o}(\varepsilon)\right)}\right)
$$

Notemos também que, se

$$
L=\sup \left\{\left|\partial^{\alpha} \hat{f}_{i}(\varepsilon, s, x)\right|:(s, x) \in \overline{I_{a}\left(t_{o}\right)} \times \overline{B_{b}\left(\widehat{x}_{o}(\varepsilon)\right)}, \alpha \in \mathbb{N}^{n+1},|\alpha|=1 \text { e } 1 \leq i \leq n\right\}
$$

então, pela Desigualdade do Valor Médio, temos que

$$
\|\widehat{f}(\varepsilon, t, y)-\widehat{f}(\varepsilon, t, z)\| \leq n^{2} L\|y-z\|,
$$

para todo $(t, y, z) \in \overline{I_{a}\left(t_{o}\right)} \times \overline{B_{b}\left(\hat{x}_{o}(\varepsilon)\right)} \times \overline{B_{b}\left(\widehat{x}_{o}(\varepsilon)\right)}$.

Como vale (2) existe $m \in \mathbb{N}$ tal que $T^{m}$ é uma contração([11]-pag 58), e assim como $\mathcal{C}\left(\overline{I_{a}\left(t_{o}\right)} ; \overline{B_{b}\left(\widehat{x}_{o}(\varepsilon)\right)}\right)$ munido da distância

$$
d(h, g)=\|h-g\|=\sup \left\{\|h(t)-g(t)\|: t \in \overline{I_{a}\left(t_{o}\right)}\right\}
$$

é um espaço métrico completo, temos que $T$ tem um único ponto fixo que chamaremos de $u_{\varepsilon}$.

Portanto, dado $\varepsilon \in] 0, \eta\left[\right.$ existe $u_{\varepsilon} \in \mathcal{C}\left(\overline{I_{a}\left(t_{o}\right)} ; \overline{B_{b}\left(\widehat{x}_{o}(\varepsilon)\right)}\right)$ tal que

$$
u_{\varepsilon}(t)=\widehat{x}_{o}(\varepsilon)+\int_{t_{o}}^{t} \hat{f}\left(\varepsilon, s, u_{\varepsilon}(s)\right) d s, \text { para todo } t \in I_{a}\left(t_{o}\right),
$$

e como $\widehat{f}(\varepsilon,.) \in \mathcal{C}^{\infty}\left(I_{a}\left(t_{o}\right) \times \Omega ; \mathbb{R}^{n}\right)$ temos que $u_{\varepsilon} \in \mathcal{C}^{\infty}\left(I_{a}\left(t_{o}\right) ; \mathbb{R}^{n}\right)$, para todo $\left.\varepsilon \in\right] 0, \eta[$. 
Seja $\widehat{u}$ a aplicação definida em $] 0,1] \times I_{a}\left(t_{o}\right)$ e com valores em $\mathbb{R}^{n}$ dada por

$$
\widehat{u}(\varepsilon, t)=\left\{\begin{array}{ll}
u_{\varepsilon}(t) & \text { se } \varepsilon \in] 0, \eta[ \\
u_{\frac{\eta}{2}}(t) & \text { se } \varepsilon \in[\eta, 1]
\end{array} .\right.
$$

Então

$$
\left.\widehat{u}(] 0,1] \times I_{a}\left(t_{o}\right)\right) \subset \cup_{\varepsilon \in] 0, \eta[} \overline{B_{b}\left(\hat{x}_{o}(\varepsilon)\right)} \subset \bar{V} \subset \subset \Omega
$$

e ainda, para todo $\varepsilon \in] 0,1]$, temos que $\hat{\imath}(\varepsilon,.) \in \mathcal{C}^{\infty}\left(I_{a}\left(t_{o}\right) ; \mathbb{R}^{n}\right)$,

$$
\begin{aligned}
& \widehat{u}(\varepsilon, t)=\widehat{x}_{o}(\varepsilon)+\int_{t_{o}}^{t} \widehat{f}(\varepsilon, s, \widehat{u}(\varepsilon, s)) d s, \text { para todo } t \in I_{a}\left(t_{o}\right), \\
& \widehat{u}\left(\varepsilon, t_{o}\right)=\widehat{x}_{o}(\varepsilon) \text { e } \widehat{u}^{\prime}(\varepsilon, .)=\widehat{f}(\varepsilon, ., \widehat{u}(\varepsilon, .)) .
\end{aligned}
$$

Provaremos, a seguir, que $\widehat{u}=\left(\widehat{u}_{1}, \ldots, \widehat{u}_{n}\right) \in \mathcal{E}_{M}\left[I_{a}\left(t_{0}\right) ; \mathbb{R}^{n}\right]$.

Sejam $J \subset \subset I_{a}\left(t_{o}\right)$ e $m \in \mathbb{N}$. Provaremos, usando o Princípio de Indução Finita sobre $m$, que existem $N \in \mathbb{I}, c>0$ e $\bar{\eta} \in] 0, \eta[$ tais que

$$
\left.\left|\widehat{u}_{i}^{(m)}(\varepsilon, t)\right| \leq c \varepsilon^{-N} \text {, para todo }(\varepsilon, t) \in\right] 0, \bar{\eta}[\times J \quad \text { e } 1 \leq i \leq n .
$$

Se $m=0$, então por (1), (4) e (i), basta tomar $N=0, \bar{\eta}=\eta$ e $c=M a+\sup \{\|z\|$ : $z \in K\}$, sendo $K$ como em (i).

Se $m=1$, então por (1) e (5), basta escolher $N=0, \bar{\eta}=\eta$ e $c=M$.

Seja $m \geq 2$. Suponhamos (6) verdadeira com $s$ no lugar de $m$, sendo $s \in \mathbb{N}$ e $s \leq m-1$. Provaremos que (6) vale para $m$.

Como $\hat{f} \in \mathcal{E}_{M}\left[I_{a}\left(t_{o}\right) \times \Omega ; \mathbb{R}^{n}\right]$ e $J \times \bar{V} \subset \subset I_{a}\left(t_{o}\right) \times \Omega$, existem $N_{1} \in \mathbb{N}, c_{1}>0$ e $\left.\eta_{1} \in\right] 0, \eta[$ tais que, se $1 \leq i \leq n$, então

$\left|\partial^{\gamma} \widehat{f}_{i}(\varepsilon, t, y)\right| \leq c_{1} \varepsilon^{-N_{1}}$, para todo $\left.(\varepsilon, t, y) \in\right] 0, \eta_{1}\left[\times J \times \bar{V}\right.$ e $\gamma \in \mathbb{N}^{n+1}$ com $|\gamma| \leq m-1$, e portanto, por (3), temos que, se $1 \leq i \leq n$ e $\gamma \in \mathbb{N}^{n+1}$, então $\left|\partial^{\gamma} \widehat{f}_{i}(\varepsilon, t, \widehat{u}(\varepsilon, t))\right| \leq c_{1} \varepsilon^{-N_{1}}$, para todo $\left.(\varepsilon, t) \in\right] 0, \eta_{1}[\times J$ e $|\gamma| \leq m-1$.

Usando a hipótese de indução, existem $N_{2} \in \mathbb{I}, c_{2}>0$ e $\left.\eta_{2} \in\right] 0, \eta_{1}[$ tais que $\left|\widehat{u}_{i}^{(s)}(\varepsilon, t)\right| \leq c_{2} \varepsilon^{-N_{2}}$, para todo $\left.(\varepsilon, t) \in\right] 0, \eta_{2}[\times J, 1 \leq i \leq n$ e $s \in \mathbb{N}$ com $s \leq m-1$.

Fixemos $\varepsilon \in] 0, \eta_{2}[$ e sejam 


$$
A=\left\{\partial^{\gamma} \widehat{f}_{i}(\varepsilon, t, \widehat{u}(\varepsilon, t)): t \in J, 1 \leq i \leq n \text { e } \gamma \in \mathbb{N}^{n+1} \operatorname{com}|\gamma| \leq m-1\right\}
$$

$\mathrm{e}$

$$
B=\left\{\widehat{u}_{i}^{(s)}(\varepsilon, t): t \in J, 1 \leq i \leq n \text { e } s \in \mathbb{N} \operatorname{com} s \leq m-1\right\}
$$

De (5) temos que, se $1 \leq j \leq n$, então

$$
\widehat{u}_{j}^{(m)}=\left(\widehat{f}_{j}(\varepsilon, ., \widehat{u}(\varepsilon, .))\right)^{(m-1)},
$$

e $\operatorname{assim} \widehat{u}_{j}^{(m)}(\varepsilon, t)$ é soma de produtos de elementos que estão em $A \cup B$, e portanto, usando (7) e (8), existem $N_{3} \in \mathbb{N}$ e $c_{3}>0$ tais que

$$
\left.\left|\widehat{u}_{j}^{(m)}(\varepsilon, t)\right| \leq c_{3} \varepsilon^{-N_{3}} \text { para todo }(\varepsilon, t) \in\right] 0, \eta_{3}[\times J \text { e } 1 \leq j \leq n
$$

o que prova (6) para $m$.

Portanto $\widehat{u} \in \mathcal{E}_{M}\left[I_{a}\left(t_{o}\right) ; \mathbb{R}^{n}\right]$.

Seja $u$ a classe de $\widehat{u} \operatorname{em~} \mathcal{G}\left(I_{a}\left(t_{o}\right) ; \mathbb{R}^{n}\right)$.

Para obter (I) basta usar (3), e por (5) concluímos (II) e (III). //

\subsubsection{Observação. A condição 3.1.9.i é necessária.}

De fato, se existe $u \in \mathcal{G}_{*}\left(I_{a}\left(t_{o}\right) ; \Omega\right)$ com $u\left(t_{o}\right)=x_{o}$, então existe um representante $\widehat{u}$ de $u$ como em 1.1.18.i e existe $h \in(\mathcal{N}(\mathbb{R}))^{n} \operatorname{com} \widehat{u}\left(\varepsilon, t_{o}\right)=\widehat{x}_{o}(\varepsilon)+h(\varepsilon)$ para todo $\left.\left.\varepsilon \in\right] 0,1\right]$, e assim, dados $\left\{t_{o}\right\} \subset \subset I_{a}\left(t_{o}\right)$ e $q=1$, existem $K_{1} \subset \subset \Omega, c>0$ e $\left.\left.\eta_{1} \in\right] 0,1\right]$ tais que

$$
\begin{aligned}
& \left\{\widehat{u}\left(\varepsilon, t_{o}\right)=\hat{x}_{o}(\varepsilon)+h(\varepsilon): \varepsilon \in\right] 0, \eta_{1}[\} \subset K_{1} \\
& \|h(\varepsilon)\| \leq c \varepsilon, \text { para todo } \varepsilon \in] 0, \eta_{1}[.
\end{aligned}
$$

Sejam $V$ aberto de $\Omega \operatorname{com} K_{1} \subset V \subset \bar{V} \subset \subset \Omega$ e $d$ a distância de $K_{1}$ a $\Omega \backslash V$. Então

$$
K_{1} \subset \cup_{x \in K_{1}} B_{\frac{d}{8}}(x) \subset \cup_{x \in K_{1}} B_{\frac{d}{2}}(x) \subset \Omega .
$$

Como $K_{1}$ é um compacto existem $y_{1}, \ldots, y_{s}$ em $K_{1}$ tais que

$$
K_{1} \subset \cup_{i=1}^{s} B_{\frac{d}{8}}\left(y_{i}\right) \subset \cup_{i=1}^{s} \overline{B_{\frac{d}{4}}\left(y_{i}\right)} \subset \cup_{i=1}^{s} B_{\frac{d}{2}}\left(y_{i}\right) \subset \Omega .
$$

Sejam $K=\cup_{i=1}^{s} \overline{B_{\frac{d}{4}}\left(y_{i}\right)} \subset \subset \Omega$ e $\eta=\min \left\{\eta_{1}, \frac{d}{8 c}\right\}$. Então, por (1) e (3), dado $\left.\varepsilon \in\right] 0, \eta[$ 
existe $j \in\{1, \ldots, s\}$ tal que $\widehat{x}_{o}(\varepsilon)+h(\varepsilon) \in B_{\frac{d}{8}}\left(y_{j}\right)$, e assim, usando (2), temos que $\left\|\widehat{x}_{o}(\varepsilon)-y_{j}\right\|=\left\|\widehat{x}_{o}(\varepsilon)+h(\varepsilon)-y_{j}-h(\varepsilon)\right\| \leq\left\|\widehat{x}_{o}(\varepsilon)+h(\varepsilon)-y_{j}\right\|+\|h(\varepsilon)\|<\frac{d}{8}+c \varepsilon \leq \frac{d}{4}$. Portanto $\left\{\widehat{x}_{o}(\varepsilon): \varepsilon \in\right] 0, \eta[\} \subset \cup_{i=1}^{s} \overline{B_{\frac{d}{4}}\left(y_{i}\right)}=K \subset \subset \Omega$.

Para obter um teorema de unicidade utilizaremos o Lema de Gronwall que afirma o seguinte:

3.1.11 Lema(Lema de Gronwall). Sejam $I=[a, b]$ um intervalo fechado de $\mathbb{R}, t_{0} \in I$, $u \in \mathcal{C}(I ; \mathbb{R})$ e $\alpha$ e $\beta$ pertencentes a $\mathbb{R}_{+}^{*}$ tais que

$$
|u(t)| \leq\left|\int_{t_{o}}^{t}(\alpha|u(s)|+\beta) d s\right|, \text { para todo } t \in[a, b] .
$$

Então

$$
|u(t)| \leq \frac{\beta}{\alpha} \max \left\{\exp \left(\alpha\left(b-t_{o}\right)\right)-1, \exp \left(\alpha\left(t_{o}-a\right)\right)-1\right\}, \text { para todo } t \in[a, b] .
$$

Demonstração. Ver [12], página 39, escolhendo para $v$ a função $v(t)=\int_{t_{o}}^{t}(\alpha|u(s)|+\beta) d s$ e dividindo a prova em dois casos. No primeiro caso, $t \geq t_{o}$, fazer como [12], e no segundo caso, $t \leq t_{o}$, substituir, em [12], $\exp (-\alpha t)$ por $\exp (\alpha t) \cdot / /$

Além do Lema de Gronwall utilizaremos o seguinte resultado:

3.1.12 Lema. Sejam $\Omega$ um aberto de $\mathbb{R}^{n}, K \subset \subset \Omega$ e $\left(\widehat{g}_{i}\right)_{i \in N^{*}} e\left(\widehat{h}_{i}\right)_{i \in N^{*}}$ seqüências de elementos de $\mathcal{E}_{M}[\Omega ; \mathbb{R}]$ tais que, dados quaisquer $i \in \mathbb{N}^{*}$ e $p \in \mathbb{N}$, existem $c>0$ e $\eta \in] 0,1]$ satisfazendo

$$
\left.\left|\widehat{g}_{i}(\varepsilon, x)-\widehat{h}_{i}(\varepsilon, x)\right| \leq c \varepsilon^{p}, \text { para todo }(\varepsilon, x) \in\right] 0, \eta[\times K
$$

Então para todo $A \subset \mathbb{N}^{*}$ finito tem-se que, dado qualquer $q \in \mathbb{N}$, existem $\bar{c}>0 e$ $\bar{\eta} \in] 0,1]$ satisfazendo

$$
\left.\left|\prod_{i \in A} \hat{g}_{i}(\varepsilon, x)-\prod_{i \in A} \widehat{h}_{i}(\varepsilon, x)\right| \leq \bar{c} \varepsilon^{q}, \text { para todo }(\varepsilon, x) \in\right] 0, \bar{\eta}[\times K
$$


Demonstração. Seja $A \subset \mathbb{N}^{*}$. Provaremos o resultado usando o Princípio de Indução Finita sobre a $\operatorname{card} A$.

Se $\operatorname{card} A=1$, então o resultado é imediato.

Suponhamos $\operatorname{card} A=k$ e que o lema seja verdadeiro com $B$ no lugar de $A$, sendo $B$ um subconjunto finito de $\mathbb{N}^{*} \operatorname{com} \operatorname{card} B<k$.

Notemos que se $A=\left\{i_{1}, \ldots, i_{k}\right\} \subset \mathbb{N}^{*}$ e $B=\left\{i_{2}, \ldots, i_{k}\right\}$, então

$$
\prod_{j \in A} \widehat{g}_{j}-\prod_{j \in A} \hat{h}_{j}=\left(\hat{g}_{i_{1}}-\widehat{h}_{i_{1}}\right) \prod_{j \in B} \hat{g}_{j}+\widehat{h}_{i_{1}}\left(\prod_{j \in B} \hat{g}_{j}-\prod_{j \in B} \hat{h}_{j}\right)
$$

Tomemos $q \in \mathbb{N}$. Como $\widehat{g}_{j} \in \mathcal{E}_{M}[\Omega ; \mathbb{R}]$ para todo $j \in \mathbb{N}^{*}$ e $\mathcal{E}_{M}[\Omega ; \mathbb{R}]$ é uma álgebra temos que $\prod_{j \in B} \hat{g}_{j} \in \mathcal{E}_{M}[\Omega ; \mathbb{R}]$.

Como $\prod_{j \in B}^{j \in B} \widehat{g}_{j} \in \mathcal{E}_{M}[\Omega ; \mathbb{R}]$ e $\widehat{h}_{i_{1}} \in \mathcal{E}_{M}[\Omega ; \mathbb{R}]$ existem $N_{1} \in \mathbb{N}, c_{1}>0$ e $\left.\left.\eta_{1} \in\right] 0,1\right]$ tais que

$$
\left|\prod_{j \in B} \widehat{g}_{j}(\varepsilon, x)\right| \leq c_{1} \varepsilon^{-N_{1}} \quad \text { e } \quad\left|\widehat{h}_{i_{1}}(\varepsilon, x)\right| \leq c_{1} \varepsilon^{-N_{1}}
$$

para todo $(\varepsilon, x) \in] 0, \eta_{1}[\times K$.

Usando a hipótese e a hipótese de indução temos que, dado $p \in \mathbb{N} \operatorname{com} p>q+N_{1}$, existem $c_{2}>0$ e $\left.\eta_{2} \in\right] 0, \eta_{1}[$ tais que

$$
\left|\widehat{g}_{i_{1}}(\varepsilon, x)-\widehat{h}_{i_{1}}(\varepsilon, x)\right| \leq c_{2} \varepsilon^{p} \quad \text { e }\left|\prod_{j \in B} \hat{h}_{j}(\varepsilon, x)-\prod_{j \in B} \hat{g}_{j}(\varepsilon, x)\right| \leq c_{2} \varepsilon^{p}
$$

para todo $(\varepsilon, x) \in] 0, \eta_{2}[\times K$.

Portanto tomando $\bar{c}=2 c_{1} c_{2}$ e $\bar{\eta}=\eta_{2}$ temos, por (1), (2) e (3) que

$$
\begin{aligned}
\left|\prod_{j \in A} \hat{g}_{j}(\varepsilon, x)-\prod_{j \in A} \hat{h}_{j}(\varepsilon, x)\right| \leq & \left|\widehat{g}_{i_{1}}(\varepsilon, x)-\hat{h}_{i_{1}}(\varepsilon, x)\right| \prod_{j \in B}\left|\widehat{g}_{j}(\varepsilon, x)\right|+ \\
& +\left|\widehat{h}_{i_{1}}(\varepsilon, x)\right|\left|\prod_{j \in B} \widehat{g}_{j}(\varepsilon, x)-\prod_{j \in B} \widehat{h}_{j}(\varepsilon, x)\right| \\
\leq & c_{2} \varepsilon^{p} c_{1} \varepsilon^{-N_{1}}+c_{1} \varepsilon^{-N 1} c_{2} \varepsilon^{p} \\
\leq & 2 c_{1} c_{2}\left(\varepsilon^{N_{1}+q} \varepsilon^{-N_{1}}\right) \leq \bar{c} \varepsilon^{q}
\end{aligned}
$$

para todo $(\varepsilon, x) \in] 0, \bar{\eta}[\times K . / /$

No próximo resultado apresentamos um teorema de unicidade de soluções para um 
sistema de E.D.O. no contexto das funções generalizadas.

3.1.13 Teorema(Unicidade). Sejam $\Omega$ um aberto de $\mathbb{R}^{n}$, I um intervalo aberto de $\mathbb{R}$, $t_{o} \in I, x_{o} \in \overline{\mathbb{R}}^{n}, f \in \mathcal{G}\left(I \times \Omega ; \mathbb{R}^{n}\right)$ e $\hat{f}$ um representante de $f$ satisfazendo:

(i) $\hat{f}$ tem a propriedade (LLL) em $(I, \Omega)$.

Se existem u e $v$ pertencentes a $\mathcal{G}_{*}(I ; \Omega)$ tais que

(ii) $u^{\prime}=f \circ\left(\pi_{*}, u\right) \quad e \quad u\left(t_{o}\right)=x_{o}$;

(iii) $v^{\prime}=f \circ\left(\pi_{*}, v\right) \quad e \quad v\left(t_{o}\right)=x_{o}$,

então $u=v$.

Demonstração. Sejam $\left(I_{j}\right)_{j \in N}$ uma seqüência exaustiva de compactos para $I$ com $t_{o} \in$ $\cap_{j \in N} I_{j}$ e $I_{j}$ intervalo para todo $j \in \mathbb{N}, \widehat{u}$ um representante de $u$ e $\hat{v}$ um representante de $v$.

Fixemos $j \in \mathbb{N}$. Provaremos que $\left.(\widehat{v}-\widehat{u})\right|_{I_{j}} \in \mathcal{N}\left[\check{I}_{j} ; \mathbb{R}^{n}\right]$, e $\operatorname{assim} u=v$ (1.1.20.I).

Como $u \in \mathcal{G}_{*}(I ; \Omega), v \in \mathcal{G}_{*}(I ; \Omega)$ e $I_{j} \subset \subset I$, existem $K_{j} \subset \subset \Omega$ e $\left.\left.\eta_{j} \in\right] 0,1\right]$ tais que

$$
\widehat{u}(] 0, \eta_{j}\left[\times I_{j}\right) \cup \widehat{v}(] 0, \eta_{j}\left[\times I_{j}\right) \subset K_{j} .
$$

Usando (ii), (iii), (1) e 1.1.21.II existem $\widehat{g}=\left(\widehat{g}_{1}, \ldots, \widehat{g}_{n}\right) \in \mathcal{N}\left[\stackrel{\circ}{I}_{j} ; \mathbb{R}^{n}\right]$ e $h=\left(h_{1}, \ldots h_{n}\right) \in$ $(\mathcal{N}(\mathbb{R}))^{n}$ tais que

$$
\widehat{g}(\varepsilon, t)=\widehat{v}^{\prime}(\varepsilon, t)-\widehat{f}(\varepsilon, t, \widehat{v}(\varepsilon, t))-\widehat{u}^{\prime}(\varepsilon, t)+\widehat{f}(\varepsilon, t, \widehat{u}(\varepsilon, t))
$$

e

$$
h(\varepsilon)=\widehat{v}\left(\varepsilon, t_{o}\right)-\widehat{u}\left(\varepsilon, t_{o}\right),
$$

para todo $t \in \stackrel{\circ}{I}_{j}$ e $\left.\quad \varepsilon \in\right] 0, \eta_{j}[$.

Portanto para todo $t \in \stackrel{\circ}{I}_{j}$ e $\left.\varepsilon \in\right] 0, \eta_{j}[$, tem-se que

$$
\begin{aligned}
\widehat{v}(\varepsilon, t)-\widehat{u}(\varepsilon, t) & =\widehat{v}\left(\varepsilon, t_{o}\right)-\widehat{u}\left(\varepsilon, t_{o}\right)+\int_{t_{o}}^{t}(\widehat{f}(\varepsilon, s, \widehat{v}(\varepsilon, s))-\widehat{f}(\varepsilon, s, \widehat{u}(\varepsilon, s))) d s+\int_{t_{o}}^{t} \widehat{g}(\varepsilon, s) d s \\
& =h(\varepsilon)+\int_{t_{o}}^{t}(\widehat{f}(\varepsilon, s, \widehat{v}(\varepsilon, s))-\widehat{f}(\varepsilon, s, \widehat{u}(\varepsilon, s))) d s+\int_{t_{o}}^{t} \widehat{g}(\varepsilon, s) d s .
\end{aligned}
$$


Seja $J \subset \subset \stackrel{\circ}{I}_{j}$. Provaremos que,

se $m \in \mathbb{N}$, então dado $q \in \mathbb{N}$ existem $c>0$ e $\eta \in] 0,1]$ tais que

$\left|\widehat{v}_{i}^{m}(\varepsilon, t)-\widehat{u}_{i}^{m}(\varepsilon, t)\right| \leq c \varepsilon^{q}$, para todo $\left.t \in J, \varepsilon \in\right] 0, \eta[$ e $1 \leq i \leq n$.

Provaremos (2) usando o Princípio de Indução Finita sobre $m$. Antes porém faremos algumas considerações.

Sejam $a$ e $b$ números reais tais que $J \cup\left\{t_{o}\right\} \subset[a, b] \subset \subset \stackrel{\circ}{I}_{j}$.

Como $\hat{f}=\left(\widehat{f}_{1}, \ldots, \widehat{f}_{n}\right) \in \mathcal{E}_{M}\left[I \times \Omega ; \mathbb{R}^{n}\right]$ e vale (i) podemos, para $K_{j}$ como em (1) e $m \in \mathbb{N}$, encontrar $N \in \mathbb{N}, \bar{c}>0$ e $\tau \in] 0, \eta_{j}[$ tais que

$$
\begin{gathered}
\left|\widehat{f}_{i}(\varepsilon, t, y)-\hat{f}_{i}(\varepsilon, t, z)\right| \leq \ln \left(\bar{c} \varepsilon^{-N}\right)\|y-z\|, \\
\left|\partial^{\beta} \widehat{f}_{i}(\varepsilon, t, y)\right| \leq \bar{c} \varepsilon^{-N} \text { e }\left|\partial^{\beta} \widehat{f}_{i}(\varepsilon, t, y)-\partial^{\beta} \widehat{f}_{i}(\varepsilon, t, z)\right| \leq \bar{c} \varepsilon^{-N}|| y-z \|,
\end{gathered}
$$

para todo $\varepsilon \in] 0, \tau\left[,(t, y, z) \in[a, b] \times K_{j} \times K_{j}, \beta \in \mathbb{N}^{n+1}\right.$ com $|\beta| \leq m$ e $1 \leq i \leq n$.

Portanto, usando (1), temos que

$$
\begin{gathered}
\left|\widehat{f}_{i}(\varepsilon, t, \widehat{u}(\varepsilon, t))-\widehat{f}_{i}(\varepsilon, t, \widehat{v}(\varepsilon, t))\right| \leq \ln \left(\bar{c} \varepsilon^{-N}\right)|| \widehat{u}(\varepsilon, t)-\widehat{v}(\varepsilon, t) \|, \\
\left|\partial^{\beta} \widehat{f}_{i}(\varepsilon, t, \widehat{u}(\varepsilon, t))\right| \leq \bar{c} \varepsilon^{-N}, \quad\left|\partial^{\beta} \widehat{f}_{i}(\varepsilon, t, \widehat{v}(\varepsilon, t))\right| \leq \bar{c} \varepsilon^{-N}
\end{gathered}
$$

e

$$
\left|\partial^{\beta} \widehat{f}_{i}(\varepsilon, t, \widehat{u}(\varepsilon, t))-\partial^{\beta} \widehat{f}_{i}(\varepsilon, t, \widehat{v}(\varepsilon, t))\right| \leq \bar{c} \varepsilon^{-N}\|\widehat{u}(\varepsilon, t)-\widehat{v}(\varepsilon, t)\|,
$$

para todo $\varepsilon \in] 0, \tau\left[, t \in[a, b], \beta \in \mathbb{N}^{n+1}\right.$ com $|\beta| \leq m$ e $1 \leq i \leq n$.

Seja $N_{1} \in \mathbb{N} \operatorname{com} N_{1}>\max \left\{N n\left(b-t_{o}\right), N n\left(t_{o}-a\right)\right\}$.

Como $h \in\left(\mathcal{N}\left(\mathbb{R}^{n}\right)\right)^{n}$ e $\hat{g} \in \mathcal{N}\left[i_{j} ; \mathbb{R}^{n}\right]$, dado $q \in \mathbb{N}$, existem $\bar{c}_{1}>0$ e $\left.\tau_{1} \in\right] 0, \tau[$ tais que

$$
\left|h_{i}(\varepsilon)\right| \leq \bar{c}_{1} \varepsilon^{q+N_{1}} \leq \bar{c}_{1} \varepsilon^{q} \quad \text { e } \quad\left|\widehat{g}_{i}^{(s)}(\varepsilon, t)\right| \leq \bar{c}_{1} \varepsilon^{q+N_{1}} \leq \bar{c}_{1} \varepsilon^{q},
$$

para todo $\varepsilon \in] 0, \tau_{1}[, t \in[a, b], s \in \mathbb{N}$ com $s \leq m$ e $1 \leq i \leq n$.

Seja $\left.\tau_{2} \in\right] 0, \tau_{1}\left[\right.$ tal que $0<\frac{n \ln \left(\bar{c} \varepsilon^{-N}\right)+1}{\ln \left(\bar{c} \varepsilon^{-N}\right)}<2 n$, para todo $\left.\varepsilon \in\right] 0, \tau_{2}[$. Provaremos, a seguir, a afirmação (2).

Suponhamos $m=0$ e tomemos $q \in \mathbb{N}$. 
Fixemos $\varepsilon \in] 0, \tau_{2}[$.

Como

$$
\widehat{v}(\varepsilon, t)-\widehat{u}(\varepsilon, t)=h(\varepsilon)+\int_{t_{o}}^{t}(\hat{f}(\varepsilon, s, \widehat{v}(\varepsilon, s))-\hat{f}(\varepsilon, s, \widehat{u}(\varepsilon, s))+\widehat{g}(\varepsilon, s)) d s,
$$

para todo $t \in[a, b]$, e valem (3) e (6), temos que

$$
\begin{aligned}
\|\widehat{v}(\varepsilon, t)-\widehat{u}(\varepsilon, t)-h(\varepsilon)\| \leq & \left|\int_{t_{o}}^{t}(\|\widehat{f}(\varepsilon, s, \widehat{v}(\varepsilon, s))-\widehat{f}(\varepsilon, s, \widehat{u}(\varepsilon, s))\|+|| \widehat{g}(\varepsilon, s) \|) d s\right| \\
\leq & \left|\int_{t_{o}}^{t} \sum_{i=1}^{n}\left(\left|\hat{f}_{i}(\varepsilon, s, \widehat{v}(\varepsilon, s))-\widehat{f_{i}}(\varepsilon, s, \widehat{u}(\varepsilon, s))\right|+\left|\widehat{g}_{i}(\varepsilon, s)\right|\right) d s\right| \\
\leq & \left|\int_{t_{o}}^{t}\left(n \ln \left(\bar{c} \varepsilon^{-N}\right)\|\widehat{v}(\varepsilon, s)-\widehat{u}(\varepsilon, s)\|+n \bar{c}_{1} \varepsilon^{q+N_{1}}\right) d s\right| \\
\leq & \mid \int_{t_{o}}^{t}\left(n \ln \left(\bar{c} \varepsilon^{-N}\right)\|\widehat{v}(\varepsilon, s)-\widehat{u}(\varepsilon, s)-h(\varepsilon)\|+\right. \\
& \left.+n \ln \left(\bar{c} \varepsilon^{-N}\right)\|h(\varepsilon)\|+n \bar{c}_{1} \varepsilon^{q+N_{1}}\right) d s \mid \\
\leq & \mid \int_{t_{o}}^{t}\left(n \ln \left(\bar{c} \varepsilon^{-N}\right)|| \widehat{v}(\varepsilon, s)-\widehat{u}(\varepsilon, s)-h(\varepsilon) \|+\right. \\
& \left.+\left(n \ln \left(\bar{c} \varepsilon^{-N}\right)+1\right) n \bar{c}_{1} \varepsilon^{q+N_{1}}\right) d s \mid,
\end{aligned}
$$

para todo $t \in[a, b]$.

Portanto, pelo Lema de Gronwall (3.1.11), concluímos que, se $S$ e $d$ são dados por

$$
\begin{aligned}
& S=\max \left\{\exp \left(n \ln \left(\bar{c} \varepsilon^{-N}\right)\left(b-t_{o}\right)\right)-1, \exp \left(n \ln \left(\bar{c} \varepsilon^{-N}\right)\left(t_{o}-a\right)\right)-1\right\} ; \\
& \bar{d}=\max \left\{\bar{c}^{n\left(b-t_{o}\right)}, \bar{c}^{n\left(t_{o}-a\right)}\right\},
\end{aligned}
$$

então

$$
\begin{aligned}
\|\hat{v}(\varepsilon, t)-\widehat{u}(\varepsilon, t)-h(\varepsilon)\| & \leq \frac{\left(n \ln \left(\bar{c} \varepsilon^{-N}\right)+1\right) n \bar{c}_{1} \varepsilon^{q+N_{1}}}{n \ln \left(\bar{c} \varepsilon^{-N}\right)} S \\
& \leq \frac{\left(n \ln \left(\bar{c} \varepsilon^{-N}\right)+1\right) \bar{c}_{1} \varepsilon^{q+N_{1}}}{\ln \left(\bar{c} \varepsilon^{-N}\right)}\left(\bar{d} \varepsilon^{-N_{1}}-1\right) \\
& \leq \frac{\left(n \ln \left(\bar{c} \varepsilon^{-N}\right)+1\right) \bar{c}_{1} \varepsilon^{q+N_{1}}}{\ln \left(\bar{c} \varepsilon^{-N}\right)} \bar{d}^{-N_{1}} \leq 2 n \bar{c}_{1} \bar{d} \varepsilon^{q},
\end{aligned}
$$

para todo $t \in[a, b]$, e assim, por (6),

$$
\|\widehat{v}(\varepsilon, t)-\widehat{u}(\varepsilon, t)\| \leq\|h(\varepsilon)\|+2 n \bar{c}_{1} \bar{d}_{\varepsilon^{q}} \leq n \bar{c}_{1} \varepsilon^{q}+2 n \bar{c}_{1} \bar{d} \varepsilon^{q} \leq\left(n \bar{c}_{1}+2 n \bar{c}_{1} \bar{d}\right) \varepsilon^{q},
$$

para todo $t \in[a, b]$. 
Como $\left|\widehat{v}_{i}(\varepsilon,)-.\widehat{u}_{i}(\varepsilon,).\right| \leq\|\widehat{v}(\varepsilon,)-.\widehat{u}(\varepsilon,)$.$\| temos que$

$\left|\widehat{v}_{i}(\varepsilon, t)-\widehat{u}_{i}(\varepsilon, t)\right| \leq\left(n \bar{c}_{1}+2 n \bar{c}_{1} \bar{d}\right) \varepsilon^{q}$, para todo $\left.(\varepsilon, t) \in\right] 0, \tau_{2}[\times[a, b]$ e $1 \leq i \leq n$,

o que prova (2) para $m=0$.

Suponhamos $m>0$ e (2) verdadeiro com $s$ no lugar de $m$, sendo $s \in \mathbb{N}$ e $s<m$. Provaremos que vale (2j para $m$.

Seja $q \in \mathbb{N}$.

Usando que $\widehat{u}$ e $\widehat{v}$ pertencem a $\mathcal{E}_{M}\left[I ; \mathbb{R}^{n}\right]$, existem $\left.\bar{c}_{2}>0, \tau_{3} \in\right] 0, \tau\left[\right.$ e $N_{2} \in \mathbb{N}$ com $N_{2}>\max \left\{N_{1}, N\right\}$ (onde, como vimos anteriormente, $\tau$ e $N$ são tais que valem (4) e (5), e $\left.N_{1}>\max \left\{N n\left(b-t_{o}\right), N n\left(t_{o}-a\right)\right\}\right)$, satisfazendo

$$
\prod_{(s, k) \in \Lambda \times A}\left|\widehat{u}_{k}^{(s)}(\varepsilon, t)\right| \leq \bar{c}_{2} \varepsilon^{-N_{2}} \quad \text { e } \prod_{(s, k) \in \Lambda \times A}\left|\widehat{v}_{k}^{(s)}(\varepsilon, t)\right| \leq \bar{c}_{2} \varepsilon^{-N_{2}},
$$

para todo $\Lambda \subset\{0,1, \ldots, m-1\}, A \subset\{1, \ldots, n\}$ e $(\varepsilon, t) \in] 0, \tau_{3}[\times[a, b]$.

Pela hipótese de indução sabemos que (2) é verdadeiro para $s \in \mathbb{N}$ com $s<m$ e assim, por 3.1.12, concluímos que, dado $2 N_{2}+q$, existem $\bar{c}_{3}>0$ e $\left.\tau_{4} \in\right] 0, \tau_{3}$ [ tais que

$$
\left|\prod_{(s, k) \in \Lambda \times A} \widehat{u}_{k}^{(s)}(\varepsilon, t)-\prod_{(s, k) \in \Lambda \times A} \widehat{v}_{k}^{(s)}(\varepsilon, t)\right| \leq \bar{c}_{3} \varepsilon^{2 N_{2}+q},
$$

para todo $\Lambda \subset\{0,1, \ldots, m-1\}, A \subset\{1, \ldots, n\}$ e $(\varepsilon, t) \in] 0, \tau_{4}[\times[a, b]$.

Fixemos $\varepsilon \in] 0, \tau_{4}[$ e $i \in\{1, \ldots, n\}$.

De (7) temos que

$$
\widehat{v}_{i}(\varepsilon, t)-\widehat{u}_{i}(\varepsilon, t)=h_{i}(\varepsilon)+\int_{t_{o}}^{t}\left(\widehat{f}_{i}(\varepsilon, s, \widehat{v}(\varepsilon, s))-\widehat{f}_{i}(\varepsilon, s, \widehat{u}(\varepsilon, s))+\widehat{g}_{i}(\varepsilon, s)\right) d s
$$

e portanto, se

$$
\widehat{w}_{1}(\varepsilon, t)=\widehat{f}_{i}(\varepsilon, t, \widehat{v}(\varepsilon, t)) \quad \text { e } \quad \widehat{w}_{2}(\varepsilon, t)=\widehat{f}_{i}(\varepsilon, t, \widehat{u}(\varepsilon, t)),
$$

então

$$
\widehat{v}_{i}^{(m)}(\varepsilon, t)-\widehat{u}_{i}^{(m)}(\varepsilon, t)=\widehat{g}_{i}^{(m-1)}(\varepsilon, t)+\widehat{w}_{1}^{(m-1)}(\varepsilon, t)-\widehat{w}_{2}^{(m-1)}(\varepsilon, t), \text { para todo } t \in[a, b] .
$$

Notemos que $\widehat{w}_{1}^{(m-1)}(\varepsilon, t)-\widehat{w}_{2}^{(m-1)}(\varepsilon, t)=\bar{a}+\bar{b}$, sendo 


$$
\bar{a}=\partial^{\gamma} \widehat{f}_{i}(\varepsilon, t, \widehat{v}(\varepsilon, t))-\partial^{\gamma} \widehat{f}_{i}(\varepsilon, t, \widehat{u}(\varepsilon, t)), \operatorname{com} \gamma=(m-1,0, \ldots, 0) \in \mathbb{N}^{n+1}
$$

e $\bar{b}$ soma de elementos do tipo

$$
a_{\beta \Lambda A}=\partial^{\beta} \widehat{f}_{i}(\varepsilon, t, \widehat{v}(\varepsilon, t)) \prod_{(s, k) \in \Lambda \times A} \widehat{v}_{k}^{(s)}(\varepsilon, t)-\partial^{\beta} \widehat{f}_{i}(\varepsilon, t, \widehat{u}(\varepsilon, t)) \prod_{(s, k) \in \Lambda \times A} \widehat{u}_{k}^{(s)}(\varepsilon, t),
$$

$\operatorname{com} \beta \in \mathbb{N}^{n+1},|\beta|<m, \Lambda \subset\{1, \ldots, m-1\}$ e $A \subset\{1, \ldots, n\}$.

Usando que (11) pode ser escrita como $b_{\beta \Lambda A}+c_{\beta \Lambda A}$, sendo

$$
b_{\beta \Lambda A}=\left[\partial^{\beta} \widehat{f}_{i}(\varepsilon, t, \widehat{v}(\varepsilon, t))-\partial^{\beta} \hat{f}_{i}(\varepsilon, t, \widehat{u}(\varepsilon, t))\right] \prod_{(s, k) \in \Lambda \times A} \widehat{v}_{k}^{(s)}(\varepsilon, t)
$$

$\mathrm{e}$

$$
c_{\beta \Lambda A}=\partial^{\beta} \widehat{f}_{i}(\varepsilon, t, \widehat{u}(\varepsilon, t))\left[\prod_{(s, k) \in \Lambda \times A} \widehat{v}_{k}^{(s)}(\varepsilon, t)-\prod_{(s, k) \in \Lambda \times A} \widehat{u}_{k}^{(s)}(\varepsilon, t)\right]
$$

e que valem (5) e (9), temos que

$$
\begin{aligned}
|\bar{a}| & \leq \bar{c} \varepsilon^{-N}\|\widehat{v}(\varepsilon, t)-\widehat{u}(\varepsilon, t)\| \\
& \leq \sum_{i=1}^{n} \bar{c} \varepsilon^{-N}\left|\widehat{v}_{i}(\varepsilon, t)-\widehat{u}_{i}(\varepsilon, t)\right| \\
& \leq n \bar{c}_{3} \bar{c} \varepsilon^{-N} \varepsilon^{2 N_{2}+q} \\
& \leq n \bar{c}_{3} \bar{c} \varepsilon^{q}
\end{aligned}
$$

e, por (4), (5), (8) e (9) temos que

$$
\begin{aligned}
\left|a_{\beta \Lambda A}\right| & \leq\left|b_{\beta \Lambda A}\right|+\left|c_{\beta \Lambda A}\right| \\
& \leq \bar{c} \varepsilon^{-N} \| \widehat{v}(\varepsilon, t)-\widehat{u}(\varepsilon, t)|| \bar{c}_{2} \varepsilon^{-N_{2}}+\bar{c}_{3} \bar{c} \varepsilon^{-N} \varepsilon^{2 N_{2}+q} \\
& \leq \sum_{i=1}^{n} \bar{c} \varepsilon^{-N}\left|\widehat{v}_{i}(\varepsilon, t)-\widehat{u}_{i}(\varepsilon, t)\right| \bar{c}_{2} \varepsilon^{-N_{2}}+\bar{c}_{3} \bar{c} \varepsilon^{N_{2}+q} \\
& \leq\left(n \bar{c}_{2} \bar{c}_{3} \bar{c} \varepsilon^{-N}+\bar{c}_{3} \bar{c}\right) \varepsilon^{N_{2}+q} \\
& \leq\left(n \bar{c}_{2} \bar{c}_{3} \bar{c}+\bar{c}_{3} \bar{c}\right) \varepsilon^{q}
\end{aligned}
$$

para todo $t \in[a, b]$.

Portanto existe $\bar{c}_{4}>0$ tal que

$$
\left|\widehat{w}_{1}^{(m-1)}(\varepsilon, t)-\widehat{w}_{2}^{(m-1)}(\varepsilon, t)\right| \leq \bar{c}_{4} \varepsilon^{q},
$$

para todo $(\varepsilon, t) \in] 0, \tau_{4}[\times[a, b]$. 
Como

$$
\left|\widehat{v}_{i}^{(m)}(\varepsilon, t)-\widehat{u}_{i}^{(m)}(\varepsilon, t)\right| \leq\left|\widehat{g}_{i}^{(m-1)}(\varepsilon, t)\right|+\left|\widehat{w}_{1}^{(m-1)}(\varepsilon, t)-\widehat{w}_{2}^{(m-1)}(\varepsilon, t)\right|
$$

temos, por (6) e (12), que existe $\bar{c}_{5}>0$ tal que

$$
\left.\left|\widehat{v}_{i}^{(m)}(\varepsilon, t)-\widehat{u}_{i}^{(m)}(\varepsilon, t)\right| \leq \bar{c}_{5} \varepsilon^{q}, \text { para todo } t \in[a, b] \text { e } \varepsilon \in\right] 0, \tau_{4}[
$$

Portanto, como $1 \leq i \leq n$ é arbitrário, temos que (2) é verdadeiro para $m$, e assim $\left.\left(\widehat{u}_{i}-\widehat{v}_{i}\right)\right|_{I_{j}} \in \mathcal{N}\left[\stackrel{\circ}{I}_{j} ; \mathbb{R}\right]$ para todo $1 \leq i \leq n$, provando que $\left.(\widehat{u}-\widehat{v})\right|_{I_{j}} \in \mathcal{N}\left[\stackrel{\circ}{I}_{j} ; \mathbb{R}^{n}\right] . / /$

Com 3.1.9 e 3.1 .13 obtemos o seguinte teorema:

3.1.14 Teorema(Existência e Unicidade). Sejam $\Omega$ um aberto de. $\mathbb{R}^{n}$, I um intervalo aberto de $\mathbb{R}, t_{o} \in I, x_{o} \in \overline{\mathbb{R}}^{n}, f \in \mathcal{G}\left(I \times \Omega ; \mathbb{R}^{n}\right), \widehat{x}_{o}$ um representante de $x_{o}$ e $\hat{f}$ um representante de $f$ satisfazendo:

(i) existe $K \subset \subset \Omega$ e $\tau \in] 0,1]$ tais que $\widehat{x}_{o}(] 0, \tau[) \subset K$;

(ii) $\hat{f}$ tem a propriedade (LLL) em $(I, \Omega)$.

Se $f \in \mathcal{G}_{*}\left(I \times \Omega ; \mathbb{R}^{n}\right)$, então existe $a>0$ com $\overline{I_{a}\left(t_{o}\right)} \subset I$ tal que existe uma única função $u \in \mathcal{G}_{*}\left(I_{a}\left(t_{o}\right) ; \Omega\right)$ satisfazendo

$$
u^{\prime}=f \circ\left(\pi_{*}, u\right) \quad \text { e } \quad u\left(t_{0}\right)=x_{0} \cdot / /
$$

A definição abaixo é análoga à usual da teoria de E.D.O.

3.1.15 Definição. Sejam $\Omega$ um aberto de $\mathbb{R}^{n}, I$ um intervalo aberto de $\mathbb{R}, f \in \mathcal{G}(I \times$ $\left.\Omega ; \mathbb{R}^{n}\right), V$ um intervalo aberto de $\mathbb{R}$ com $V \subset I$ e $u \in \mathcal{G}_{*}(V ; \Omega)$ uma solução da equação

$$
w^{\prime}=f \circ\left(\pi_{*}, w\right) .
$$

Dizemos que $v \in \mathcal{G}_{*}(J ; \Omega)$ é um prolongamento de u se, e somente se, $v$ é uma solução da equação acima e $J$ é um intervalo aberto de $\mathbb{R} \operatorname{com} V \neq J, V \subset J \subset I$ e $\left.v\right|_{V}=u$. 
Dizemos que u é uma solução maximal de (1) se, e somente se, u não admite um prolongamento.

O próximo resultado garante, sob certas hipóteses, a existência de soluções maximais.

3.1.16 Teorema. Sejam $\Omega$ um aberto de $\mathbb{R}^{n}, I$ um intervalo aberto de $\mathbb{R}, t_{o} \in I$, $x_{o} \in \overline{\mathbb{R}}^{n}, f \in \mathcal{G}\left(I \times \Omega ; \mathbb{R}^{n}\right)$, $\widehat{x}_{o}$ um representante de $x_{o}$ e $\widehat{f}$ um representante de $f$ satisfazendo:

(i) existem $K \subset \subset \Omega$ e $\tau \in] 0,1]$ tais que $\widehat{x}_{o}(] 0, \tau[) \subset K$;

(ii) $\hat{f}$ tem a propriedade (LLL) em $(I, \Omega)$.

Se $f \in \mathcal{G}_{*}\left(I \times \Omega ; \mathbb{R}^{n}\right)$, então existe uma única solução maximal $u$ de $w^{\prime}=f \circ\left(\pi_{*}, w\right)$ tal que $u\left(t_{o}\right)=x_{o}$.

Demonstração. Por 3.1.9 existe $V$ intervalo aberto de $\mathbb{R}$ com $t_{0} \in V, V \subset I$ e existe $u \in \mathcal{G}_{*}(V ; \Omega) \operatorname{com} u^{\prime}=f \circ\left(\pi_{*}, u\right)$ e $u\left(t_{o}\right)=x_{o}$.

Seja $J$ a reunião de todos os intervalos abertos, $J_{a}$, de $\mathbb{R}$, contendo $t_{o}$ e contidos em $I$, para os quais existe $u_{a} \in \mathcal{G}_{*}\left(J_{a} ; \Omega\right) \operatorname{com} u_{a}^{\prime}=f \circ\left(\pi_{*}, u_{a}\right)$ e $u_{a}\left(t_{o}\right)=x_{o}$. Portanto podemos escrever $J=\cup_{a \in \Lambda} J_{a}$, para algum $\Lambda$. É claro que $J$ é um intervalo aberto de $\mathbb{R}$, $t_{o} \in J$ e $J \subset I$.

Usando 3.1.13 temos que

$$
\left.u_{a}\right|_{J_{a} \cap J_{b}}=\left.u_{b}\right|_{J_{a} \cap J_{b}}, \text { para todo }(a, b) \in \Lambda \times \Lambda
$$

Usando 1.1.20, existe uma única $u \in \mathcal{G}_{*}(J ; \Omega)$ tal que $\left.u\right|_{J_{a}}=u_{a}$ para todo $a \in \Lambda$.

Provaremos, em primeiro lugar, que $u$ é uma solução de $w^{\prime}=f \circ\left(\pi_{*}, \omega\right)$ e $u\left(t_{o}\right)=x_{o}$.

Seja $g=f \circ\left(\pi_{*}, u\right)$.

Como $a \in \Lambda$ e vale 1.1.24 temos

$$
\left.g\right|_{J_{a}}=\left.\left(f \circ\left(\pi_{*}, u\right)\right)\right|_{J_{a}}=f \circ\left(\left.\pi_{*}\right|_{J_{a}},\left.u\right|_{J_{a}}\right)=f \circ\left(\left.\pi_{*}\right|_{J_{a}}, u_{a}\right)=u_{a}^{\prime}=\left(\left.u\right|_{J_{a}}\right)^{\prime}=\left.u^{\prime}\right|_{J_{a}},
$$


e por 1.1.20.I concluímos que $f \circ\left(\pi_{*}, u\right)=g=u^{\prime}$, e como $u\left(t_{o}\right)=u_{a}\left(t_{o}\right)=x_{o}$, para todo $a \in \Lambda$, temos que $u$ é uma solução maximal.

Provaremos, a seguir, que existe uma única solução maximal.

Sejam $V$ um intervalo aberto de $\mathbb{R} \operatorname{com} t_{o} \in V \subset I$ e $v \in \mathcal{G}_{*}(J ; \Omega)$ uma solução maximal de $w^{\prime}=f \circ\left(\pi_{*}, w\right)$ com $v\left(t_{o}\right)=x_{o}$. De 3.1.13 temos que $\left.u\right|_{J \cap V}=\left.v\right|_{J \cap V}$ e como $u$ e $v$ não admitem prolongamentos temos que $J=V$, e portanto $u=v \cdot / /$

Faremos, a seguir, um breve comentário sobre E.D.O. de ordem n no contexto das funções generalizadas de Colombeau.

Sejam $\Omega$ um aberto de $\mathbb{R}^{n}, I$ um intervalo aberto de $\mathbb{R}, t_{o} \in I, \widehat{f} \in \mathcal{E}_{M}[I \times \Omega ; \mathbb{R}], \hat{g}$ a aplicação moderada definida em $] 0,1] \times I \times \Omega$ por

$$
\hat{g}\left(\varepsilon, t, y_{1}, \ldots, y_{n}\right)=\left(y_{2}, \ldots, y_{n}, \hat{f}\left(\varepsilon, t, y_{1}, \ldots, y_{n}\right)\right)
$$

$f$ a classe de $\hat{f}$ em $\mathcal{G}(I \times \Omega ; \mathbb{R})$ e $g$ a classe de $\hat{g}$ em $\mathcal{G}\left(I \times \Omega ; \mathbb{R}^{n}\right)$.

Consideremos os seguintes problemas:

(1) determinar $u \in \mathcal{G}(I ; \mathbb{R}) \operatorname{com}\left(u, u^{\prime}, \ldots, u^{(n-1)}\right) \in \mathcal{G}_{*}(I ; \Omega)$ e tal que

$$
\left(u\left(t_{o}\right), u^{\prime}\left(t_{o}\right), \ldots, u^{(n-1)}\left(t_{o}\right)\right)=x_{o} \in \overline{\mathbb{R}}^{n} \text { e } u^{(n)}=f \circ\left(\pi_{*}, u, u^{\prime}, \ldots, u^{(n-1)}\right) ;
$$

(2) determinar $v=\left(v_{1}, \ldots, v_{n}\right) \in \mathcal{G}_{*}(I ; \Omega) \operatorname{com} v\left(t_{o}\right)=x_{\circ}$ e $v^{\prime}=g \circ\left(\pi_{*}, v\right)$.

Notemos que para resolver (1) basta resolver (2) e escolher $u=v_{1}$, e que se $u$ é uma solução de (1), então $v=\left(u, u^{\prime}, \ldots, u^{(n-1)}\right)$ é uma solução de (2). Com esta observação e $3.1 .9,3.1 .13,3.1 .14$ e 3.1.16 obtemos resultados para E.D.O. de ordem n, no contexto das funções generalizadas.

Para finalizar esta seção apresentamos a seguir exemplos de funções que satisfazem as hipóteses do teorema de existência e unicidade. 
3.1.17 Exemplo. Sejam I um intervalo aberto de $\mathbb{R}, \Omega$ um aberto convexo de $\mathbb{R}^{n}$, $\varphi=\left(\varphi_{1}, \ldots, \varphi_{n}\right) \in \mathcal{C}^{\infty}\left(I \times \Omega ; \mathbb{R}^{n}\right), \Psi=\left(\Psi_{1}, \ldots, \Psi_{n}\right) \in \mathcal{C}^{\infty}\left(\mathbb{R} ; \mathbb{R}^{n}\right)$ e $\Phi=\left(\Phi_{1}, \ldots, \Phi_{n}\right) \in$ $\mathcal{C}^{\infty}\left(\mathbb{R} ; \mathbb{R}^{n}\right)$ tais que $\Phi_{i}$ e todas as derivadas de $\Phi_{i}$ são funções limitadas, para todo $1 \leq i \leq n$. Então a aplicação $f$ de 3.1 .14 pode ser substituída por uma das classes das aplicaçôes moderadas definidas em $] 0,1] \times I \times \Omega$ por

$$
\begin{gathered}
\widehat{g}(\varepsilon, t, x)=\left(\Psi_{1}\left(\varepsilon \varphi_{1}(t, x)\right), \ldots, \Psi_{\mathrm{n}}\left(\varepsilon \varphi_{n}(t, x)\right)\right) \\
\widehat{h}(\varepsilon, t, x)=\left(\Phi_{1}\left(\ln \left(\frac{1}{\varepsilon}\right) \varphi_{1}(t, x)\right), \ldots, \Phi_{\mathrm{n}}\left(\ln \left(\frac{1}{\varepsilon}\right) \varphi_{n}(t, x)\right)\right) .
\end{gathered}
$$

De fato, denotando por $(t, x)=\left(t, x_{1}, \ldots, x_{n}\right)$ um ponto genérico de $\mathbb{R}^{n+1}$, basta usar $3.1 .6 \mathrm{e}$ observar que, se $\mu$ é a função definida em $] 0,1]$ por $\mu(\varepsilon)=\varepsilon$ ou $\mu(\varepsilon)=\ln \left(\frac{1}{\varepsilon}\right), 1 \leq i \leq n$, $\Gamma=\Psi_{i}$ ou $\Phi_{i}$ e $l$ é a função definida em $\left.] 0,1\right] \times I \times \Omega$ por $l(\varepsilon, t, x)=\Gamma\left(\mu(\varepsilon) \varphi_{i}(t, x)\right)$, então dados $(\varepsilon, t, x)$ e $(\varepsilon, t, y)$ pertencentes a $] 0,1] \times I \times \Omega$ tem-se, usando o Teorema do Valor Médio, que

$$
\begin{aligned}
|l(\varepsilon, t, x)-l(\varepsilon, t, y)| & =\left|\Gamma\left(\mu(\varepsilon) \varphi_{i}(t, x)\right)-\Gamma\left(\mu(\varepsilon) \varphi_{i}(t, y)\right)\right| \\
& \leq \mu(\varepsilon) \sum_{j=1}^{n}\left|\Gamma^{\prime}\left(\mu(\varepsilon) \varphi_{i}(\bar{P})\right) \frac{\partial \varphi_{i}}{\partial x_{j}}(\bar{P})\left(x_{j}-y_{j}\right)\right|
\end{aligned}
$$

onde $\bar{P}$ pertence ao segmento de extremidades $(t, x)$ e $(t, y)$.

(As funções seno e cosseno podem ser usadas, por exemplo, para $\Phi_{i}$.)

\subsection{Equações diferenciais ordinárias : dependência}

\section{das soluções relativamente a parâmetros}

Quando estudamos E.D.O., no caso clássico, após obter teoremas de existência e unicidade de soluções locais para a equação vetorial $w^{\prime}(t)=f(t, w(t))$ com $w\left(t_{o}\right)=x_{0}$ passa-se a analisar o seguinte problema (que é conhecido como dependência contínua das soluções relativamente a parâmetros): Dados $I$ um intervalo aberto de $\mathbb{R}, t_{o} \in I, \Omega$ um aberto de $\mathbb{R}^{n}, \Omega^{\prime}$ um aberto de $\mathbb{R}^{m}, x_{0} \in \mathbb{R}^{n}$ e $f \in \mathcal{C}^{1}\left(I \times \Omega \times \Omega^{\prime} ; \mathbb{R}^{n}\right)$, existem a>0 com $\overline{I_{a}\left(t_{o}\right)} \subset I, W$ um aberto de $\mathbb{R}^{n}$ e $u \in \mathcal{C}\left(I_{a}\left(t_{o}\right) \times W ; \mathbb{R}^{n}\right)$ satisfazendo: 


$$
\frac{\partial u}{\partial t}(t, y)=f(t, u(t, y), y) \quad \text { e } \quad u\left(t_{o}, .\right)=x_{o} \quad ?
$$

Nesta seção analisaremos, em primeiro lugar, o problema acima generalizado, isto é, admitindo $f \in \mathcal{G}\left(I \times \Omega \times \Omega^{\prime} ; \mathbb{R}^{n}\right)$ e $x_{o} \in \overline{\mathbb{R}}^{n}$. Neste caso, as equações que aparecem em (1) são escritas na forma

$$
\frac{\partial u}{\partial t}=f \circ\left(\pi, u, \pi_{1}, \ldots, \pi_{m}\right) \quad \text { e }\left.\quad u\right|_{\left\{t_{o}\right\} \times W}=x_{o}
$$

sendo $u \in \mathcal{G}_{*}\left(I_{a}\left(t_{o}\right) \times W ; \Omega\right)$. As funções $\pi, \pi_{1}, \ldots, \pi_{m}$ foram definidas no início do capítulo 2. A seguir estudaremos (2) substituindo o vetor generalizado $x_{o}$ por uma função generalizada.

Os principais resultados desta seção estão a partir de 3.2.12. Além das notações anteriores, utilizaremos, para facilitar a escrita de alguns dos resultados, a seguinte definição:

3.2.1 Definição. Sejam $\Omega$ um aberto de $\mathbb{R}^{n}$, I um intervalo aberto de $\mathbb{R}, t_{o} \in I, a>0$ com $\overline{I_{a}\left(t_{o}\right)} \subset I, \Omega^{\prime}$ um aberto de $\mathbb{R}^{m}, W$ um aberto de $\Omega^{\prime}$ e $\left.\left.\tau \in\right] 0,1\right]$. Denotaremos por

$$
\mathcal{E}\left(t_{o}, a, \tau, I, \Omega, \Omega^{\prime}, W\right)
$$

o conjunto das ternas $\left(\widehat{x}_{o}, \widehat{f}, \widehat{u}\right)$ tais que

(i) $\widehat{x}_{o}$ é uma aplicação definida em $\left.] 0,1\right]$ e com valores em $\mathbb{R}^{n}$;

(ii) $\hat{f}$ é uma aplicação definida em $] 0,1] \times I \times \Omega \times \Omega^{\prime}$ e com valores em $\mathbb{R}^{n}$;

(iii) $\widehat{u}$ é uma aplicação definida em $] 0,1] \times I_{a}\left(t_{o}\right) \times W$ e com valores em $\mathbb{R}^{n}$;

(iv) $\widehat{f}(\varepsilon,.) \in \mathcal{C}^{\infty}\left(I \times \Omega \times \Omega^{\prime} ; \mathbb{R}^{n}\right)$, para todo $\left.\varepsilon \in\right] 0, \tau[$;

(v) $\widehat{u}(\varepsilon,.) \in \mathcal{C}^{\infty}\left(I_{a}\left(t_{o}\right) \times W ; \mathbb{R}^{n}\right)$, para todo $\left.\varepsilon \in\right] 0, \tau[$;

(vi) existe $K \subset \subset \Omega$ tal que $\widehat{u}(] 0, \tau\left[\times I_{a}\left(t_{o}\right) \times W\right) \subset K \subset \subset \Omega$;

(vii) $\frac{\partial \widehat{u}}{\partial t}(\varepsilon, t, y)=\widehat{f}(\varepsilon, t, \widehat{u}(\varepsilon, t, y), y)$, para todo $\left.(\varepsilon, t, y) \in\right] 0, \tau\left[\times I_{a}\left(t_{o}\right) \times W\right.$;

(viii) $\widehat{u}\left(\varepsilon, t_{o},.\right)=\widehat{x}_{o}(\varepsilon)$, para todo $\left.\varepsilon \in\right] 0, \tau[$. 
Usando a teoria clássica de E.D.O. podemos observar o seguinte:

3.2.2 Ọbservação. A afirmaçấo 3.2.1.v é uma conseqüência de 3.2.1.iv, 3.2.1.vii e 3.2.1.viii. De fato, fixemos $\varepsilon \in] 0, \tau\left[\right.$ e sejam $y_{o}=\widehat{x}_{o}(\varepsilon)$ e $h=\widehat{f}(\varepsilon,$.$) . Então a aplicação v=\widehat{u}(\varepsilon,)$. é uma solução da equação vetorial

$$
\frac{\partial w}{\partial t}(t, y)=h(t, w(t, y), y) \quad \operatorname{com} \quad w\left(t_{o}, .\right)=y_{o},
$$

e sabemos, da teoria clássica de E.D.O.[10], que a solução do problema acima é $\mathcal{C}^{\infty}$ quando $h \in \mathcal{C}^{\infty}$. Portanto $\widehat{u}(\varepsilon,.) \in \mathcal{C}^{\infty}\left(I_{a}\left(t_{o}\right) \times W ; \mathbb{R}^{n}\right)$.

Apesar da observação acima, optamos por manter a asserção 3.2.1.v para facilitar a escrita da prova de alguns dos resultados.

Notemos também que para resolver (2) (definido no início desta seção) basta determinar $\hat{v} \operatorname{com}\left(\hat{x}_{o}, \hat{f}, \widehat{v}\right) \in \mathcal{E}\left(t_{o}, a, \tau, I, \Omega, \Omega^{\prime}, W\right)$, sendo $\hat{f}$ um representante de $f$, e tal que a aplicação $\widehat{u}$ definida em $] 0,1] \times I_{a}\left(t_{o}\right) \times W$ por

$$
\left.\widehat{u}\right|_{] 0, \tau\left[\times I_{a}\left(t_{o}\right) \times W\right.}=\widehat{v} \quad \text { e }\left.\quad \widehat{u}\right|_{[\tau, 1] \times I_{a}\left(t_{o}\right) \times W} \quad \text { é constante }
$$

pertença a $\mathcal{E}_{M}\left[I_{a}\left(t_{o}\right) \times W ; \mathbb{R}^{n}\right]$. De fato, se $u$ é a classe de $\widehat{u}$ em $\mathcal{G}\left(I_{a}\left(t_{o}\right) \times W ; \mathbb{R}^{n}\right)$, então 3.2.1.vi garante que $u \in \mathcal{G}_{*}\left(I_{a}\left(t_{o}\right) \times W ; \Omega\right)$ e com 3.2.1.vii e 3.2.1.viii concluímos que $u$ é uma solução procurada. Veremos a seguir que, sob certas condições, o conjunto $\mathcal{E}\left(t_{o}, a, \tau, I, \Omega, \Omega^{\prime}, W\right)$ será não vazio. Para obter $\widehat{u} \in \mathcal{E}_{M}\left[I_{a}\left(t_{o}\right) \times W ; \mathbb{R}^{n}\right]$ além da condição $\widehat{f} \in \mathcal{E}_{M}\left[I \times \Omega \times \Omega^{\prime} ; \mathbb{R}^{n}\right]$ iremos admitir que $\hat{f}$ tenha mais uma propriedade (3.2.4.iii) e que $\bar{W} \subset \subset \Omega^{\prime}(3.2 \cdot 4 . \mathrm{i})$.

3.2.3 Proposição. Sejam $\Omega$ um aberto de $\mathbb{R}^{n}, I$ um intervalo aberto de $\mathbb{R}, t_{o} \in I, \Omega^{\prime}$ um aberto de $\mathbb{R}^{m}, W$ um aberto de $\Omega^{\prime}$, $\widehat{x}_{o}$ uma aplicação definida em 10,1$]$ e com valores em $\mathbb{R}^{n}$ e $\hat{f}$ uma aplicação definida em $\left.] 0,1\right] \times I \times \Omega \times \Omega^{\prime}$ e com valores em $\mathbb{R}^{n}$ satisfazendo:

(i) $\bar{W} \subset \subset \Omega^{\prime}$; 
(ii) existem $K_{1} \subset \subset \Omega$ e $\left.\left.\tau_{1} \in\right] 0,1\right]$ tais que $\widehat{x}_{o}(] 0, \tau_{1}[) \subset K_{1}$;

(iii) $\hat{f}(\varepsilon,.) \in \mathcal{C}^{1}\left(I \times \Omega \times \Omega^{\prime} ; \mathbb{R}^{n}\right)$, para todo $\left.\left.\varepsilon \in\right] 0,1\right]$;

(iv) dados quaisquer $J \subset \subset I, K \subset \subset \Omega$ e $K^{\prime} \subset \subset \Omega^{\prime}$, existem $\left.\left.\eta \in\right] 0,1\right]$ e $M>0$ tais que $\|\widehat{f}(\varepsilon, t, x, z)\| \leq M$, para todo $(\varepsilon, t, x, z) \in] 0, \eta\left[\times J \times K \times K^{\prime}\right.$.

Então existem $a>0$ com $\left.\left.\overline{I_{a}\left(t_{o}\right)} \subset I, \tau \in\right] 0,1\right]$ e uma aplicação $\widehat{u}$ definida em $\left.] 0,1\right] \times$ $I_{a}\left(t_{o}\right) \times W$ tais que

(I) $\widehat{u}$ satisfaz de 3.2.1.vi até 3.2.1.viii;

(II) se $\hat{f}(\varepsilon,.) \in \mathcal{C}^{\infty}\left(I \times \Omega \times \Omega^{\prime} ; \mathbb{R}^{n}\right)$ para todo $\left.\varepsilon \in\right] 0, \tau\left[\right.$, então $\left(\widehat{x}_{o}, \widehat{f}, \widehat{u}\right) \in$ $\mathcal{E}\left(t_{o}, a, \tau, I, \Omega, \Omega^{\prime}, W\right)$.

Demonstração. Sejam $K_{1}$ e $\tau_{1}$ como em (ii), $V$ um aberto de $\Omega$ tal que $K_{1} \subset V \subset \bar{V} \subset \subset \Omega$ e $d>0$ a distância de $K_{1}$ a $\Omega \backslash V$.

Seja $a^{*}>0$ tal que $\overline{I_{a^{*}}\left(t_{o}\right)} \subset I$. Então usando (iv) existem $\left.\tau \in\right] 0, \tau_{1}[$ e $M>0$ tais que

$$
\|\widehat{f}(\varepsilon, t, x, z)\| \leq M, \text { para todo }(\varepsilon, t, x, z) \in] 0, \tau\left[\times \overline{I_{a^{*}}\left(t_{o}\right)} \times \bar{V} \times \bar{W} .\right.
$$

Seja $a>0 \operatorname{com} a<\min \left\{\frac{d}{M}, a^{*}\right\}$.

Fixemos $\varepsilon \in] 0, \tau[$ e seja $T$ a função

$$
T: g \in \mathcal{C}\left(\overline{I_{a}\left(t_{o}\right)} \times \bar{W} ; \overline{B_{d}\left(\widehat{x}_{o}(\varepsilon)\right)}\right) \longmapsto T g \in \mathcal{C}\left(\overline{I_{a}\left(t_{o}\right)} \times \bar{W} ; \mathbb{R}^{n}\right)
$$

onde $T g$ é a aplicação definida em $\overline{I_{a}\left(t_{o}\right)} \times \bar{W}$ por

$$
(T g)(t, y)=\widehat{x}_{o}(\varepsilon)+\int_{t_{o}}^{t} \widehat{f}(\varepsilon, s, g(s, y), y) d s
$$

Usando (1) temos que

$$
\left\|(T g)(t, y)-\widehat{x}_{o}(\varepsilon)\right\| \leq\left|\int_{t_{o}}^{t}\|\widehat{f}(\varepsilon, s, g(s, y), y)\| d s\right| \leq M\left|t-t_{o}\right| \leq M a<d
$$

e $\operatorname{assim}(T g)(t, y) \in \overline{B_{d}\left(\hat{x}_{o}(\varepsilon)\right)} \subset \bar{V}$, para todo $(t, y) \in \overline{I_{a}\left(t_{o}\right)} \times \bar{W}$. 
Portanto

$$
T: \mathcal{C}\left(\overline{I_{a}\left(t_{o}\right)} \times \bar{W} ; \overline{B_{d}\left(\widehat{x}_{o}(\varepsilon)\right)}\right) \longmapsto \mathcal{C}\left(\overline{I_{a}\left(t_{o}\right)} \times \bar{W} ; \overline{B_{d}\left(\widehat{x}_{o}(\varepsilon)\right)}\right)
$$

Notemos também que, se $L$ é o número dado por $\sup \left\{\left|\partial^{\alpha} \hat{f}_{i}(\varepsilon, s, x, y)\right|:(s, x, y) \in \overline{I_{a}\left(t_{o}\right)} \times \overline{B_{d}\left(\widehat{x}_{o}(\varepsilon)\right)} \times \bar{W}, \alpha \in N^{n+m+1},|\alpha|=1\right.$ e $\left.1 \leq i \leq n\right\}$ que existe por (iii), então, pela Desigualdade do Valor Médio, temos que

$$
\|\widehat{f}(\varepsilon, t, x, y)-\widehat{f}(\varepsilon, t, z, y)\| \leq n^{2} L\|x-z\|,
$$

para todo $(t, x, y, z) \in \overline{I_{a}\left(t_{o}\right)} \times \overline{B_{d}\left(\widehat{x}_{o}(\varepsilon)\right)} \times \bar{W} \times \overline{B_{d}\left(\widehat{x}_{o}(\varepsilon)\right)}$.

Como vale (2) existe $m \in \mathbb{N}$ tal que $T^{m}$ é uma contração (prova análoga à de [11]-página 58), e como $\mathcal{C}\left(\overline{I_{a}\left(t_{o}\right)} \times \bar{W} ; \overline{B_{d}\left(\widehat{x}_{o}(\varepsilon)\right)}\right)$ munido da distância

$$
d(g, h)=\|h-g\|=\sup \left\{\|h(t, y)-g(t, y)\|:(t, y) \in \overline{I_{a}\left(t_{o}\right)} \times \bar{W}\right\},
$$

é um espaço métrico completo, temos que $T$ tem um único ponto fixo que chamaremos de $u_{\varepsilon}$.

Portanto definindo a aplicação $\widehat{u}$ em $] 0,1] \times I_{a}\left(t_{o}\right) \times W$ por

$$
\widehat{u}(\varepsilon, t, y)=\left\{\begin{array}{ll}
u_{\varepsilon}(t, y) & \text { se } \varepsilon \in] 0, \tau[ \\
u_{\frac{\tau}{2}}(t, y) & \text { se } \varepsilon \in[\tau, 1]
\end{array},\right.
$$

e observando que $\left.\widehat{u}(] 0,1] \times I_{a}\left(t_{o}\right) \times W\right)=\cup_{\varepsilon \in] 0,1]} \overline{B_{d}\left(\widehat{x}_{o}(\varepsilon)\right)} \subset \bar{V} \subset \subset \Omega$, temos que $\hat{u}$ satisfaz de 3.2.1.vi até 3.2.1.viii, o que prova (I).

A asserção (II) é uma conseqüência de (I) e de 3.2.2 . //

3.2.4 Proposição. Sejam $\Omega$ um aberto de $\mathbb{R}^{n}$, I um intervalo aberto de $\mathbb{R}, t_{0} \in I$, $a>0$ com $\overline{I_{a}\left(t_{o}\right)} \subset I, \Omega^{\prime}$ um aberto de $\mathbb{R}^{m}, W$ um aberto de $\left.\left.\Omega^{\prime}, \tau \in\right] 0,1\right]$ e $\left(\widehat{x}_{o}, \widehat{f}, \widehat{u}\right) \in$ $\mathcal{E}\left(t_{o}, a, \tau, I, \Omega, \Omega^{\prime}, W\right)$. Denotando por $(t, x, y)=\left(t, x_{1}, \ldots, x_{n}, y_{1}, \ldots, y_{m}\right)$ um ponto genérico de $\mathbb{R} \times \mathbb{R}^{n} \times \mathbb{R}^{m}$ tem-se que, se

(i) $\bar{W} \subset \subset \Omega^{\prime}$; 
(ii) $\hat{f} \in \mathcal{E}_{M}\left[I \times \Omega \times \Omega^{\prime} ; \mathbb{R}^{n}\right]$;

(iii) $\frac{\partial \widehat{f}_{i}}{\partial x_{j}}$ tem a propriedade (CLL) em $I \times \Omega \times \Omega^{\prime}$, para todo $1 \leq i, j \leq n$, então dados quaisquer $J \subset \subset I_{a}\left(t_{o}\right), K^{\prime} \subset \subset W$ e $\beta \in N^{m+1}$, existem $\left.c>0, \eta \in\right] 0, \tau[$ e $N \in \mathbb{N}$ satisfazendo

$$
\left.\left|\partial^{\beta} \widehat{u}_{i}(\varepsilon, t, y)\right| \leq c \varepsilon^{-N}, \quad \text { para todo }(\varepsilon, t, y) \in\right] 0, \eta\left[\times J \times K^{\prime} \text { e } 1 \leq i \leq n .\right.
$$

Demonstração. Denotaremos por $(t, x, y)=\left(t, x_{1}, \ldots, x_{n}, y_{1}, \ldots, y_{m}\right)$ um ponto genérico de $\mathbb{R} \times \mathbb{R}^{n} \times \mathbb{R}^{m}$ e por $(t, y)=\left(t, y_{1}, \ldots, y_{m}\right)$ um ponto genérico de $\mathbb{R} \times \mathbb{R}^{m}$.

Sejam $J \subset \subset I_{a}\left(t_{o}\right)$ e $K^{\prime} \subset \subset W$. Provaremos a proposição usando o Príncipio de Indução Finita sobre $|\beta|$, sendo $\beta \in \mathbb{N}^{m+1}$.

Tomemos $K \subset \subset \Omega$ como em 3.2.1.vi. Então se $\beta \in \mathbb{N}^{m+1}$ e $|\beta|=0$ basta, por 3.2.1.vi, fazer $N=0, \eta=\tau$ e $c=\sup \{\|y\|: y \in K\}$.

Seja $p \in \mathbb{N}$. Suponhamos a proposição verdadeira para todo $\beta \in \mathbb{N}^{m+1}$ com $|\beta| \leq p$.

Seja $\beta \in \mathbb{N}^{m+1}$ com $|\beta|=p+1>0$.

Usando (ii) e (iii) existem $c>0, \eta \in] 0, \tau[$ e $N \in \mathbb{N}$ tais que

$$
\left|\partial^{\alpha} \widehat{f}_{i}(\varepsilon, s, x, y)\right| \leq c \varepsilon^{-N} \quad \text { e } \quad\left|\frac{\partial \widehat{f}_{i}}{\partial x_{j}}(\varepsilon, s, x, y)\right| \leq \ln \left(c \varepsilon^{-N}\right),
$$

para todo $(\varepsilon, s, x, y) \in] 0, \eta\left[\times \overline{I_{a}\left(t_{o}\right)} \times K \times \bar{W}, \alpha \in \mathbb{N}^{n+m+1} \quad\right.$ com $|\alpha| \leq p+1 \quad \mathrm{e}$ $1 \leq i, j \leq n$, e assim usando 3.2.1.vi temos que

$$
\begin{gathered}
\left.\left|\frac{\partial \widehat{f}_{i}}{\partial x_{j}}(\varepsilon, s, \widehat{u}(\varepsilon, s, y), y)\right| \leq \ln \left(c \varepsilon^{-N}\right), \text { para todo }(\varepsilon, s, y) \in\right] 0, \eta\left[\times I_{a}\left(t_{o}\right) \times W\right. \\
\left.\left|\partial^{\alpha} \widehat{f}_{i}(\varepsilon, s, \widehat{u}(\varepsilon, s, y), y)\right| \leq c \varepsilon^{-N}, \quad \text { para todo }(\varepsilon, s, y) \in\right] 0, \eta\left[\times I_{a}\left(t_{o}\right) \times W\right.
\end{gathered}
$$

para todo $\alpha \in \mathbb{N}^{n+m+1}$ com $|\alpha| \leq p+1$ e $1 \leq i, j \leq n$.

Para $\varepsilon \in] 0, \eta[$ sejam

$$
\begin{aligned}
& A_{\varepsilon}=\left\{\partial^{\alpha} \widehat{f}_{k}(\varepsilon, s, \widehat{u}(\varepsilon, s, y), y):(s, y) \in I_{a}\left(t_{o}\right) \times W, \alpha \in \mathbb{N}^{n+m+1} \operatorname{com}|\alpha| \leq p+1 \text { e } 1 \leq k \leq n\right\} \\
& \mathrm{e} \\
& B_{\varepsilon}=\{1\} \cup\left\{\partial^{\gamma} \widehat{u}_{k}(\varepsilon, s, y):(s, y) \in I_{a}\left(t_{o}\right) \times W, \gamma \in \mathbb{N}^{m+1} \operatorname{com}|\gamma| \leq p \text { e } 1 \leq k \leq n\right\} .
\end{aligned}
$$


Suponhamos $\beta=\left(\beta_{0}, \beta_{1}, \ldots, \beta_{m}\right)$ e, em primeiro lugar, $\beta_{0}=0$.

Seja $j \in\{1, \ldots, m\}$ tal que $\beta_{j} \neq 0$. Como $\widehat{u}(\varepsilon,.) \in \mathcal{C}^{\infty}\left(I_{a}\left(t_{o}\right) \times W ; \mathbb{R}^{n}\right)$ temos que

$$
\left.\frac{\partial^{2} \widehat{u}}{\partial y_{j} \partial t}(\varepsilon, .)=\frac{\partial^{2} \widehat{u}}{\partial t \partial y_{j}}(\varepsilon, .), \text { para todo } \varepsilon \in\right] 0, \eta[
$$

e assim por 3.2.1.vii e 3.2.1.viii concluímos que

$$
\frac{\partial \widehat{u}_{i}}{\partial y_{j}}(\varepsilon, t, y)=\int_{t_{o}}^{t}\left(\sum_{k=1}^{n} \frac{\partial \widehat{f}_{i}}{\partial x_{k}}(\varepsilon, s, \widehat{u}(\varepsilon, s, y), y) \frac{\partial \widehat{u}_{k}}{\partial y_{j}}(\varepsilon, s, y)+\frac{\partial \widehat{f}_{i}}{\partial y_{j}}(\varepsilon, s, \widehat{u}(\varepsilon, s, y), y)\right) d s,
$$

para todo $(\varepsilon, t, y) \in] 0, \eta\left[\times I_{a}\left(t_{o}\right) \times W, \quad 1 \leq i \leq n\right.$ e $1 \leq j \leq m$.

Então derivando (3) sob o sinal de integração temos

$$
\partial^{\beta} \widehat{u}_{i}(\varepsilon, t, y)=\partial^{\bar{\beta}}\left(\frac{\partial \widehat{u}_{i}}{\partial y_{j}}(\varepsilon, t, y)\right)=\int_{t_{o}}^{t}\left(l_{1}(\varepsilon, s, y)+l_{2}(\varepsilon, s, y)\right) d s
$$

onde $\bar{\beta}=\beta-e_{j+1}$, sendo $e_{j+1}$ oj+1-ésimo elemento da base canônica de $\mathbb{R}^{m+1}, l_{1}(\varepsilon, s, y)$ é soma de produtos de elementos de $A_{\varepsilon} \cup B_{\varepsilon}$ e

$$
l_{2}(\varepsilon, s, y)=\sum_{k=1}^{n} \frac{\partial \widehat{f}_{i}}{\partial x_{k}}(\varepsilon, s, \widehat{u}(\varepsilon, s, y), y) \partial^{\beta} \widehat{u}_{k}(\varepsilon, s, y),
$$

para todo $(\varepsilon, t, y) \in] 0, \eta\left[\times I_{a}\left(t_{o}\right) \times W, \quad 1 \leq i \leq n\right.$ e $1 \leq j \leq m$.

Sejam $a_{1}$ e $b_{1}$ números reais tais que $J \cup\left\{t_{o}\right\} \subset\left[a_{1}, b_{1}\right] \subset I_{a}\left(t_{o}\right)$.

Usando (2) e a hipótese de indução, existem $c_{1}>0, N_{1} \in \mathbb{I}$ e $\left.\eta_{1} \in\right] 0, \eta[$ tais que

$$
\left.\left|l_{1}(\varepsilon, s, y)\right| \leq c_{1} \varepsilon^{-N_{1}} \text {, para todo }(\varepsilon, s, y) \in\right] 0, \eta_{1}\left[\times\left[a_{1}, b_{1}\right] \times K^{\prime} .\right.
$$

Portanto se $\varepsilon \in] 0, \eta_{1}[$ temos, por (1), que

$$
\begin{aligned}
\left|\partial^{\beta} \widehat{u}_{i}(\varepsilon, t, y)\right| & \leq\left|\int_{t_{o}}^{t}\left(c_{1} \varepsilon^{-N_{1}}+\sum_{k=1}^{n}\left|\frac{\partial \widehat{f}_{i}}{\partial x_{k}}(\varepsilon, s, \widehat{u}(\varepsilon, s, y), y)\right|\left|\partial^{\beta} \widehat{u}_{k}(\varepsilon, s, y)\right|\right) d s\right| \\
& \leq\left|\int_{t_{o}}^{t}\left(c_{1} \varepsilon^{-N_{1}}+n \ln \left(c \varepsilon^{-N}\right)||\left(\partial^{\beta} \widehat{u}_{1}(\varepsilon, s, y), \ldots, \partial^{\beta} \widehat{u}_{n}(\varepsilon, s, y)\right)||\right) d s\right|,
\end{aligned}
$$

e assim denotando por $\partial^{\beta} \widehat{u}$ a n-upla $\left(\partial^{\beta} \widehat{u}_{1}, \ldots, \partial^{\beta} \widehat{u}_{n}\right)$ temos

$$
\left\|\partial^{\beta} \widehat{u}(\varepsilon, t, y)\right\| \leq \sum_{i=1}^{n}\left|\partial^{\beta} \widehat{u}_{i}(\varepsilon, t, y)\right| \leq\left|\int_{t_{o}}^{t}\left(n c_{1} \varepsilon^{-N_{1}}+n^{2} \ln \left(c \varepsilon^{-N}\right)|| \partial^{\beta} \widehat{u}(\varepsilon, s, y) \|\right) d s\right|,
$$

e assim, pelo Lema de Gronwall (3.1.11), concluímos que, se

$$
S=\max \left\{\exp \left(n^{2} \ln \left(c \varepsilon^{-N}\right)\left(b_{1}-t_{o}\right)\right)-1, \exp \left(n^{2} \ln \left(c \varepsilon^{-N}\right)\left(t_{o}-a_{1}\right)\right)-1\right\}
$$


então para todo $(\varepsilon, t, y) \in] 0, \eta_{1}\left[\times\left[a_{1}, b_{1}\right] \times K^{\prime}\right.$ tem-se que

$$
\left\|\partial^{\beta} \widehat{u}(\varepsilon, t, y)\right\| \leq \frac{c_{1} \varepsilon^{-N_{1}}}{n \ln \left(c \varepsilon^{-N}\right)} S .
$$

Sejam $\bar{N} \in \mathbb{N}$ com $\bar{N}>\max \left\{N_{1}, N n^{2}\left(b_{1}-t_{o}\right), N n^{2}\left(t_{o}-a_{1}\right)\right\}$, $\bar{c}=\max \left\{c_{1}, c^{n^{2}\left(b_{1}-t_{o}\right)}, c^{n^{2}\left(t_{o}-a_{1}\right)}\right\}$ e $\left.\eta_{2} \in\right] 0, \eta_{1}\left[\right.$ tal que $\min \left\{\bar{c} \varepsilon^{-\bar{N}}, n \ln \left(c \varepsilon^{-N}\right)\right\}>1$, para todo $\varepsilon \in] 0, \eta_{2}[$.

Então, por (4), tomando $c_{2}=\bar{c}^{2}$ e $N_{2}=2 \bar{N}$ temos, para todo $1 \leq i \leq n$, que

$$
\left.\left|\partial^{\beta} \widehat{u}_{i}(\varepsilon, t, y)\right| \leq\left\|\partial^{\beta} \widehat{u}(\varepsilon, t, y)\right\| \leq c_{2} \varepsilon^{-N_{2}}, \text { para todo }(\varepsilon, t, y) \in\right] 0, \eta_{2}\left[\times\left[a_{1}, b_{1}\right] \times K^{\prime}\right.
$$

o que prova o resultado nesse caso.

Finalmente suponhamos $\beta_{0} \neq 0$. Então para $\left.(\varepsilon, t, y) \in\right] 0, \eta\left[\times I_{a}\left(t_{o}\right) \times W\right.$, temos, fazendo $\bar{\beta}=\left(\beta_{0}-1, \beta_{1}, \ldots, \beta_{m}\right)$ e usando 3.2.1.vii, que

$$
\left|\partial^{\beta} \widehat{u}_{i}(\varepsilon, t, y)\right|=\left|\partial^{\bar{\beta}}\left(\frac{\partial \widehat{u}_{i}}{\partial t}\right)(\varepsilon, t, y)\right|=\left|\partial^{\bar{\beta}}\left(\widehat{f}_{i}(\varepsilon, t, \widehat{u}(\varepsilon, t, y), y)\right)\right|
$$

e assim $\left|\partial^{\beta} \widehat{u}_{i}(\varepsilon, t, y)\right|$ é soma de produtos de elementos de $A_{\varepsilon} \cup B_{\varepsilon}$.

Portanto usando (2) e a hipótese de indução concluímos que a proposição é verdadeira neste caso. //

Com a proposição acima e 3.2.3 apresentaremos em 3.2.5 um teorema de existência de soluções para a equação (2) (definida no início desta seção). Lembramos que a asserção 3.2.3.iv, quando $\hat{f} \in \mathcal{E}_{M}\left[I \times \Omega \times \Omega^{\prime} ; \mathbb{R}^{n}\right]$, é equivalente a $f \in \mathcal{G}_{*}\left(I \times \Omega \times \Omega^{\prime} ; \mathbb{R}^{n}\right)$, sendo $f$ a classe de $\hat{f}$ em $\mathcal{G}\left(I \times \Omega \times \Omega^{\prime} ; \mathbb{R}^{n}\right)$.

3.2.5 Teorema(Existência). Sejam $\Omega$ um aberto de $\mathbb{R}^{n}$, I um intervalo aberto de $\mathbb{R}$, $t_{o} \in I, \Omega^{\prime}$ um aberto de $\mathbb{R}^{m}, W$ um aberto de $\Omega^{\prime}, x_{o} \in \overline{\mathbb{R}}^{n}, f \in \mathcal{G}\left(I \times \Omega \times \Omega^{\prime} ; \mathbb{R}^{n}\right)$, $\widehat{x}_{o}$ um representante de $x_{0}$ e $\hat{f}=\left(\hat{f}_{1}, \ldots, \hat{f}_{n}\right)$ um representante de $f$. Denotando por $(t, x, y)=\left(t, x_{1}, \ldots, x_{n}, y_{1}, \ldots, y_{m}\right)$ um ponto genérico de $I \times \Omega \times \Omega^{\prime}$ tem-se que, se

(i) $\bar{W} \subset \subset \Omega^{\prime}$;

(ii) existem $K_{1} \subset \subset \Omega$ e $\left.\left.\tau_{1} \in\right] 0,1\right]$ tais que $\widehat{x}_{o}(] 0, \tau_{1}[) \subset K_{1}$; 
(iii) $f \in \mathcal{G}_{*}\left(I \times \Omega \times \Omega^{\prime} ; \mathbb{R}^{n}\right)$;

(iv) $\frac{\partial \widehat{f}_{i}}{\partial x_{j}}$ tem a propriedade (CLL) em $I \times \Omega \times \Omega^{\prime}$, para todo $1 \leq i, j \leq n$,

então existem $a>0$ com $\overline{I_{a}\left(t_{o}\right)} \subset I$, uma aplicação $u \in \mathcal{G}\left(I_{a}\left(t_{o}\right) \times W ; \mathbb{R}^{n}\right)$ e um representante $\widehat{u}$ de u satisfazendo:

(I) existe $\tau \in] 0,1] \operatorname{com}\left(\widehat{x}_{o}, \widehat{f}, \widehat{u}\right) \in \mathcal{E}\left(t_{o}, a, \tau, I, \Omega, \Omega^{\prime}, W\right)$;

(II) $u \in \mathcal{G}_{*}\left(I_{a}\left(t_{o}\right) \times W ; \Omega\right)$;

(III) $\frac{\partial u}{\partial t}=f \circ\left(\pi, u, \pi_{1}, \ldots, \pi_{m}\right) \quad$ em $\mathcal{G}\left(I_{a}\left(t_{o}\right) \times W ; \mathbb{R}^{n}\right)$;

(IV) $\left.u\right|_{\left\{t_{o}\right\} \times W}=x_{o}$.

Demonstração. Usando (i), (ii), (iii) e 3.2 .3 existem $a>0, \tau \in] 0,1]$ e uma aplicação $\widehat{v}$ definida em $] 0,1] \times I_{a}\left(t_{o}\right) \times W$ tais que $\left(\widehat{x}_{o}, \widehat{f}, \widehat{v}\right) \in \mathcal{E}\left(t_{o}, a, \tau, I, \Omega, \Omega^{\prime}, W\right)$.

Seja $\widehat{u}$ a aplicação definida em $] 0,1] \times I_{a}\left(t_{o}\right) \times W$ por

$$
\widehat{u}(\varepsilon, t, y)=\left\{\begin{array}{ll}
\widehat{v}(\varepsilon, t, y) & \text { se } \varepsilon \in] 0, \tau[ \\
\widehat{v}\left(\frac{\tau}{2}, t, y\right) & \text { se } \varepsilon \in[\tau, 1]
\end{array} .\right.
$$

Usando (i), (iv) e 3.2.4 e observando que, dado $L \subset \subset I_{a}\left(t_{o}\right) \times W$ existem $J \subset \subset I_{a}\left(t_{o}\right)$ e $K^{\prime} \subset \subset W$ tais que $L \subset J \times K^{\prime}$, temos que $\widehat{u} \in \mathcal{E}_{M}\left[I_{a}\left(t_{o}\right) \times W ; \mathbb{R}^{n}\right]$.

Seja $u$ a classe de $\widehat{u}$ em $\mathcal{G}\left(I_{a}\left(t_{o}\right) \times W ; \mathbb{R}^{n}\right)$. Então a afirmação (I) é imediata e as asserções (II), (III) e (IV) decorrem do fato de que $\left(\widehat{x}_{o}, \widehat{f}, \widehat{v}\right) \in \mathcal{E}\left(t_{o}, a, \tau, I, \Omega, \Omega^{\prime}, W\right)$, isto é, são conseqüências de 3.2.1.vi, 3.2.1.vii e 3.2.1.viii respectivamente. //

A aplicação $\widehat{u}$ encontrada na prova de 3.2 .5 foi construída utilizando uma certa função $\widehat{v}$, sendo que $\left(\widehat{x}_{o}, \widehat{f}, \widehat{v}\right) \in \mathcal{E}\left(t_{o}, a, \tau, I, \Omega, \Omega^{\prime}, W\right)$ e $\left.\widehat{u}\right|_{] 0, \tau\left[\times I_{a}\left(t_{o}\right) \times W\right.}=\widehat{v}$. Portanto para que $\widehat{u}$ tenha certas propriedades basta que $\widehat{v}$ as tenha. Por esta razão iremos ver, a seguir, algumas propriedades dos elementos de $\mathcal{E}\left(t_{o}, a, \tau, I, \Omega, \Omega^{\prime}, W\right)$, que nos serão úteis neste trabalho. 
3.2.6 Proposição. Sejam $p \in N, \Omega$ um aberto de $\mathbb{R}^{n}, I$ um intervalo aberto de $\mathbb{R}$, $t_{o} \in I, a>0$ com $\overline{I_{a}\left(t_{0}\right)} \subset I, \Omega^{\prime}$ um aberto de $\mathbb{R}^{m}, W$ um aberto de $\left.\left.\Omega^{\prime}, \tau \in\right] 0,1\right]$ e $\left(\widehat{x}_{o}, \widehat{f}, \widehat{u}\right) \in \mathcal{E}\left(t_{o}, a, \tau, I, \Omega, \Omega^{\prime}, W\right) . S e$

(i) $\bar{W} \subset \subset \Omega^{\prime}$;

(ii) dados quaisquer $J \subset \subset I, K_{1} \subset \subset \Omega, K^{\prime} \subset \subset \Omega^{\prime}$ e $\alpha \in \mathbb{N}^{n+m+1}$ com $|\alpha| \leq p$, existem $M>0 \in \eta \in] 0, \tau[$ sutisfazendo

$\left|\partial^{\alpha} \hat{f}_{i}(\varepsilon, t, x, y)\right| \leq M$, para todo $\left.(\varepsilon, t, x, y) \in\right] 0, \eta\left[\times J \times K_{1} \times K^{\prime}\right.$ e $1 \leq i \leq n$,

então

(I) dados quaisquer $J_{1} \subset \subset I_{a}\left(t_{o}\right), K^{\prime} \subset \subset W$ e $\beta \in \mathbb{N}^{m+1}$ com $|\beta| \leq p$, existem $\bar{M}>0$ e $\bar{\eta} \in] 0, \tau[$ satisfazendo

$$
\left.\left|\partial^{\beta} \widehat{u}_{i}(\varepsilon, t, y)\right| \leq \bar{M}, \text { para todo }(\varepsilon, t, y) \in\right] 0, \bar{\eta}\left[\times J_{1} \times K^{\prime} \quad e \quad 1 \leq i \leq n ;\right.
$$

(II) se (ii) é verdadeira para todo $\alpha=\left(\alpha_{0}, \alpha_{1}, \ldots, \alpha_{n+m+1}\right) \in \mathbb{N}^{n+m+1}$ com $\alpha_{0}=0 \quad e$ $|\alpha|=1$, então (i) é verdadeira para todo $\beta=\left(\beta_{0}, \beta_{1}, \ldots, \beta_{m}\right) \in \mathbb{N}^{m+1}$ com $\beta_{0}=0$

$e|\beta|=1$.

Demonstração. A prova é análoga à prova de 3.2 .4 , substituindo $\ln \left(c \varepsilon^{-N}\right)$ e $c \varepsilon^{-N}$ nas afirmações, respectivamente, (1) e (2) da demonstração de 3.2.4 por $M$ (que existe pela asserção (ii) desta proposição). //

Se a desigualdade que aparece em 3.2.6.ii for verdadeira em $J \times \Omega \times \Omega^{\prime}$, então obtemos a seguinte proposição :

3.2.7 Proposição. Sejam $p \in \mathbb{N}, \Omega$ um aberto de $\mathbb{R}^{n}, I$ um intervalo aberto de $\mathbb{R}$, $t_{o} \in I, a>0$ com $\overline{I_{a}\left(t_{0}\right)} \subset I, \Omega^{\prime}$ um aberto de $\mathbb{R}^{m}, W$ um aberto de $\left.\left.\Omega^{\prime}, \tau \in\right] 0,1\right] e$ $\left(\widehat{x}_{o}, \widehat{f}, \widehat{u}\right) \in \mathcal{E}\left(t_{o}, a, \tau, I, \Omega, \Omega^{\prime}, W\right)$. Se

(i) dados quaisquer $J \subset \subset I$ e $\alpha \in \mathbb{N}^{n+m+1}$ com $|\alpha| \leq p$, existem $M>0 \quad e$ $\eta \in] 0, \tau[$ satisfazendo 


$$
\left.\left|\partial^{\alpha} \widehat{f}_{i}(\varepsilon, t, x, y)\right| \leq M \text { para todo }(\varepsilon, t, x, y) \in\right] 0, \eta\left[\times J \times \Omega \times \Omega^{\prime} \text { e } 1 \leq i \leq n,\right.
$$

então

(I) dados quaisquer $J_{1} \subset \subset I_{a}\left(t_{0}\right)$ e $\beta \in \mathbb{N}^{m+1}$ com $|\beta| \leq p$, existem $\bar{M}>0$ e $\bar{\eta} \in] 0, \tau[$ satisfazendo

$$
\left.\left|\partial^{\beta} \widehat{u}_{i}(\varepsilon, t, y)\right| \leq \bar{M}, \text { para todo }(\varepsilon, t, y) \in\right] 0, \bar{\eta}\left[\times J_{1} \times W \quad \in 1 \leq i \leq n ;\right.
$$

(II) se (i) é verdadeira para todo $\alpha=\left(\alpha_{0}, \alpha_{1}, \ldots, \alpha_{n+m+1}\right) \in \mathbb{N}^{n+m+1}$ com $\alpha_{0}=0 \quad e$ $|\alpha|=1$, então (I) é verdadeira para todo $\beta=\left(\beta_{0}, \beta_{1}, \ldots, \beta_{m}\right) \in \mathbb{N}^{m+1}$ com $\beta_{0}=0$ e $|\beta|=1$.

Demonstração. A prova é análoga à de 3.2 .4 substituindo $\ln \left(c \varepsilon^{-N}\right)$ e $c \varepsilon^{-N}$ nas afirmações, respectivamente, (1) e (2) da demonstração de 3.2 .4 por $M$ (que existe pela asserção (i) desta proposição quando fazemos $\left.J=\overline{I_{a}\left(t_{0}\right)}\right)$ / /

Com 3.2.5, 3.2.6 e 3.2.7 concluímos que:

3.2.8 Teorema. Sejam $\Omega, I, t_{o}, \Omega^{\prime}, W, x_{o}, f, \hat{x}_{o}$ e $\hat{f}=\left(\hat{f}_{1}, \ldots, \hat{f}_{n}\right)$ satisfazendo de 3.2.5.i até 3.2.5.iv. Denotando por $(t, x, y)=\left(t, x_{1}, \ldots, x_{n}, y_{1}, \ldots, y_{m}\right)$ um ponto genérico de $\mathbb{R}^{n+m+1}$ e por $(t, y)=\left(t, y_{1}, \ldots, y_{m}\right)$ um ponto genérico de $\mathbb{R}^{m+1}$ tem-se que, existem $a>0$ com $\overline{I_{a}\left(t_{o}\right)} \subset I$, uma aplicação $u \in \mathcal{G}\left(I_{a}\left(t_{o}\right) \times W ; \mathbb{R}^{n}\right)$ e um representante $\hat{u}$ de $u$ tais que

(I) as asserçôes 3.2.5.I até 3.2.5.IV são verdadeiras;

(II) se $p \in \mathbb{N}$ e $\partial^{\alpha} f_{i} \in \mathcal{G}_{*}\left(I \times \Omega \times \Omega^{\prime} ; \mathbb{R}\right)$, para todo $\alpha \in \mathbb{N}^{n+m+1}$ com $|\alpha| \leq p$ e $1 \leq i \leq n$, então $\partial^{\beta} u_{i} \in \mathcal{G}_{*}\left(I_{a}\left(t_{o}\right) \times W ; \mathbb{R}\right)$, para todo $\beta \in \mathbb{N}^{m+1}$ com $|\beta| \leq p \quad$ e $1 \leq i \leq n ;$

(III) se $p \in \mathbb{N}$ e para quaisquer $J \subset \subset I$ e $\alpha \in \mathbb{N}^{n+m+1}$ com $|\alpha| \leq p$ tem-se que $\partial^{\alpha} \widehat{f}_{i}$ é uma função limitada em $J \times \Omega \times \Omega^{\prime}$ (ver 3.1.1.i), para todo $1 \leq i \leq n$, 
então para quaisquer $J_{1} \subset \subset I_{a}\left(t_{o}\right)$ e $\beta \in \mathbb{N}^{m+1}$ com $|\beta| \leq p$ tem-se que

$\partial^{\beta} \widehat{u}_{i}$ é uma funçâo limitada em $J_{1} \times W$, para todo $1 \leq i \leq n$;

(IV) se para todo $1 \leq i, k \leq n$ e $1 \leq j \leq m$, tem-se que

$\frac{\partial f_{i}}{\partial x_{k}}$ e $\frac{\partial f_{i}}{\partial y_{j}} \quad$ pertencem a $\mathcal{G}_{*}\left(I \times \Omega \times \Omega^{\prime} ; \mathbb{R}\right)$,

então

$\frac{\partial u_{i}}{\partial y_{j}} \in \mathcal{G}_{*}\left(I_{a}\left(t_{o}\right) \times W ; \mathbb{R}\right)$, para todo $1 \leq i \leq n$ e $1 \leq j \leq m ;$

(V) se para todo $J \subset \subset I, 1 \leq i, k \leq n$ e $1 \leq j \leq m$ tem-se que $\frac{\partial \hat{f}_{i}}{\partial x_{k}}$ e $\frac{\partial \hat{f}_{i}}{\partial y_{j}}$ são funções limitadas em $J \times \Omega \times \Omega^{\prime}$,

então para todo $J_{1} \subset \subset I_{a}\left(t_{o}\right), 1 \leq i \leq n$ e $1 \leq j \leq m$ tem-se que

$\frac{\partial \widehat{u}_{i}}{\partial y_{j}} \quad$ é uma função limitada em $J_{1} \times W \cdot / /$

Sob certas condições, como veremos nos dois próximos resultados, podemos substituir $W$ em 3.2 .8 por $\Omega^{\prime}$.

3.2.9 Teorema. Sejam $\Omega$ um aberto de $\mathbb{R}^{n}, I$ um intervalo aberto de $\mathbb{R}, t_{o} \in I, \Omega^{\prime}$ um aberto de $\mathbb{R}^{m}, x_{o} \in \overline{\mathbb{R}}^{n}, f \in \mathcal{G}\left(I \times \Omega \times \Omega^{\prime} ; \mathbb{R}^{n}\right)$, $\hat{x}_{o}$ um representante de $x_{o} e$ $\widehat{f}=\left(\hat{f}_{1}, \ldots, \hat{f}_{n}\right)$ um representante de $f$. Denotando por $(t, x, y)=\left(t, x_{1}, \ldots, x_{n}, y_{1}, \ldots, y_{m}\right)$ um ponto genérico de $\mathbb{R}^{n+m+1}$ tem-se que, se

(i) existem $K_{1} \subset \subset \Omega$ e $\left.\left.\tau_{1} \in\right] 0,1\right]$ tais que $\widehat{x}_{o}(] 0, \tau_{1}[) \subset K_{1}$;

(ii) $\hat{f}$ é uma aplicação limitada em $I \times \Omega \times \Omega^{\prime}$;

(iii) $\frac{\partial \widehat{f}_{i}}{\partial x_{j}} \quad$ tem a propriedade (CLL) em $I \times \Omega \times \Omega^{\prime}$, para todo $1 \leq i, j \leq n$, então existem $a>0$ com $\overline{I_{a}\left(t_{o}\right)} \subset I$ e uma aplicação $u \in \mathcal{G}\left(I_{a}\left(t_{o}\right) \times \Omega^{\prime} ; \mathbb{R}^{n}\right)$ satisfazendo:

(I) existem $\tau \in] 0,1]$ e $\widehat{u}$ representante de $u \operatorname{com}\left(\widehat{x}_{o}, \widehat{f}, \widehat{u}\right) \in \mathcal{E}\left(t_{o}, a, \tau, I, \Omega, \Omega^{\prime}, \Omega^{\prime}\right)$;

(II) $u \in \mathcal{G}_{*}\left(I_{a}\left(t_{o}\right) \times \Omega^{\prime} ; \Omega\right)$; 
(III) $\frac{\partial u}{\partial t}=f \circ\left(\pi, u, \pi_{1}, \ldots, \pi_{m}\right)$ em $\mathcal{G}\left(I_{a}\left(t_{o}\right) \times \Omega^{\prime} ; \mathbb{R}^{n}\right)$;

(IV) $\left.u\right|_{\left\{t_{0}\right\} \times \Omega^{\prime}}=x_{0} \quad$ em $\mathcal{G}\left(\Omega^{\prime} ; \mathbb{R}^{n}\right)$;

(V) se $p \in N$ e para todo $\alpha \in N^{n+m+1}$ com $|\alpha| \leq p$ tem-se que

$\partial^{\alpha} f_{i} \in \mathcal{G}_{*}\left(I \times \Omega \times \Omega^{\prime} ; \mathbb{R}\right)$, para todo $1 \leq i \leq n$,

então

$\partial^{\beta} u_{i} \in \mathcal{G}_{*}\left(I_{a}\left(t_{o}\right) \times \Omega^{\prime} ; \mathbb{R}\right)$, para todo $\beta \in \mathbb{N}^{m+1}$ com $|\beta| \leq p$ e $1 \leq i \leq n$;

(VI) se $p \in N$ e para todo $\alpha \in N^{n+m+1}$ com $|\alpha| \leq p$ tem-se que

$\partial^{\alpha} \hat{f}_{i}$ é uma função limitada em $J \times \Omega \times \Omega^{\prime}$, para todo $J \subset \subset I$ e $1 \leq i \leq n$, então para todo $\beta \in \mathbb{N}^{m+1}$ com $|\beta| \leq p$ tem-se que

$\partial^{\beta} \widehat{u}_{i}$ é uma função limitada em $J_{1} \times \Omega^{\prime}$, para todo $J_{1} \subset \subset I_{a}\left(t_{o}\right)$ e $1 \leq i \leq n$;

(VII) se para todo $1 \leq i, k \leq n$ e $1 \leq j \leq m$ tem-se que

$\frac{\partial f_{i}}{\partial x_{k}}$ e $\frac{\partial f_{i}}{\partial y_{j}}$ pertencem a $\mathcal{G}_{*}\left(I \times \Omega \times \Omega^{\prime} ; \mathbb{R}\right)$,

então

$\frac{\partial u_{i}}{\partial y_{j}} \in \mathcal{G}_{*}\left(I_{a}\left(t_{o}\right) \times \Omega^{\prime} ; \mathbb{R}\right)$, para todo $1 \leq i \leq n$ e $1 \leq j \leq m ;$

(VIII) se para todo $J \subset \subset I, 1 \leq i, k \leq n$ e $1 \leq j \leq m$ tem-se que

$\frac{\partial \hat{f}_{i}}{\partial x_{k}}$ e $\frac{\partial \hat{f}_{i}}{\partial y_{j}}$ são funções limitadas em $J \times \Omega \times \Omega^{\prime}$,

então para todo $J_{1} \subset \subset I_{a}\left(t_{o}\right), 1 \leq i \leq n$ e $1 \leq j \leq m$ tem-se que

$\frac{\partial \widehat{u}_{i}}{\partial y_{j}} \quad$ é uma função limitada em $J_{1} \times \Omega^{\prime}$.

Demonstraçấo. Sejam $K_{1}$ e $\tau_{1}$ como em (i), $V$ um aberto de $\Omega \operatorname{com} K_{1} \subset V \subset \bar{V} \subset \subset \Omega$, $d>0$ a distância de $K_{1}$ a $\Omega \backslash V$ e, por (ii), $M>0$ e $\left.\tau \in\right] 0, \tau_{1}[$ tais que

$$
\|\widehat{f}(\varepsilon, t, x, y)\| \leq M, \text { para todo }(\varepsilon, t, x, y) \in] 0, \tau\left[\times I \times \Omega \times \Omega^{\prime} .\right.
$$

Sejam $a^{*}>0 \operatorname{com} \overline{I_{a^{*}}\left(t_{0}\right)} \subset I$ e $a>0 \operatorname{com} a<\min \left\{\frac{d}{M}, a^{*}\right\}$. 
Fixemos $\varepsilon \in] 0, \tau[$.

Para cada $y \in \Omega^{\prime}$ sejam $r_{y}>0 \operatorname{com} \overline{B_{r_{y}}(y)} \subset \Omega^{\prime}$ e $T_{y}^{\varepsilon}$ a aplicação $T_{y}^{\varepsilon}: g \in \mathcal{C}\left(\overline{I_{a}\left(t_{o}\right)} \times \overline{B_{r_{y}}(y)} ; \overline{B_{d}\left(\hat{x}_{o}(\varepsilon)\right)}\right) \longmapsto T_{y}^{\varepsilon} g \in \mathcal{C}\left(\overline{I_{a}\left(t_{o}\right)} \times \overline{B_{r_{y}}(y)} ; \overline{B_{d}\left(\hat{x}_{o}(\varepsilon)\right)}\right)$

onde

$$
\left(T_{y}^{\varepsilon} g\right)(i, w)=\widehat{x}_{o}(\varepsilon)+\int_{t_{0}}^{t} \hat{f}(\varepsilon, s, g(s, w), w) d s .
$$

Analisando as provas de 3.2 .5 e 3.2 .3 existe uma aplicação que será denotada por $u_{r_{y}}$ tal que $u_{r_{y}} \in \mathcal{G}\left(I_{a}\left(t_{0}\right) \times B_{r_{y}}(y) ; \mathbb{R}^{n}\right)$, satisfaz de 3.2.5.II até 3.2.5.IV(substituindo $W$ por $\left.B_{r_{y}}(y)\right)$ e ainda existe um representante $\widehat{u}_{r_{y}}$ de $u_{r_{y}}$ que está definido em $\left.] 0,1\right] \times \overline{I_{a}\left(t_{o}\right)} \times$ $\overline{B_{r_{y}}(y)}, \widehat{u}_{r_{y}}(\varepsilon,).\left(\overline{I_{a}\left(t_{o}\right)} \times \overline{B_{r_{y}}(y)}\right) \subset \overline{B_{d}\left(\widehat{x}_{o}(\varepsilon)\right)} \subset \bar{V}, \widehat{u}_{r_{y}}(\varepsilon,$.$) é o único ponto fixo de T_{y}^{\varepsilon}$, para todo $\varepsilon \in] 0, \tau\left[\right.$, e $\left(\widehat{x}_{o}, \widehat{f}, \widehat{u}_{r_{y}}\right) \in \mathcal{E}\left(t_{o}, a, \tau, I, \Omega, \Omega^{\prime}, B_{r_{y}}(y)\right)$.

Notemos que, se $\varepsilon \in] 0, \tau\left[\right.$ e $y, z \in \Omega^{\prime}$ são tais que $K=\overline{B_{r_{y}}(y)} \cap \overline{B_{r_{z}}(z)} \neq \emptyset$ e $T^{\varepsilon}$ é a aplicação

$$
T^{\varepsilon}: g \in \mathcal{C}\left(\overline{I_{a}\left(t_{o}\right)} \times K ; \overline{B_{d}\left(\widehat{x}_{o}(\varepsilon)\right)}\right) \longmapsto T^{\varepsilon} g \in \mathcal{C}\left(\overline{I_{a}\left(t_{o}\right)} \times K ; \overline{B_{d}\left(\widehat{x}_{o}(\varepsilon)\right)}\right)
$$

onde

$$
\left(T^{\varepsilon} g\right)(t, w)=\widehat{x}_{o}(\varepsilon)+\int_{t_{o}}^{t} \widehat{f}(\varepsilon, s, g(s, w), w) d s,
$$

então $T^{\varepsilon}$ tem um único ponto fixo (prova análoga à de [11]-página 58) e $\left.\widehat{u}_{r_{y}}(\varepsilon,)\right|_{.\overline{I_{a}\left(t_{o}\right)} \times K}$ e $\left.\widehat{u}_{r_{z}}(\varepsilon,)\right|_{.\overline{I_{a}\left(t_{o}\right)} \times K}$ são pontos fixos de $T^{\varepsilon}$, e assim

$$
\left.u_{r_{y}}(\varepsilon, .)\right|_{\overline{I_{a}\left(t_{o}\right)} \times K}=\left.u_{r_{z}}(\varepsilon, .)\right|_{\overline{I_{a}\left(t_{o}\right)} \times K} .
$$

Portanto podemos definir a aplicação $\widehat{u} \mathrm{em}] 0,1] \times I_{a}\left(t_{o}\right) \times \Omega^{\prime}$ satisfazendo

$$
\left.\widehat{u}=\widehat{u}_{r_{y}} \text { em }\right] 0, \tau\left[\times I_{a}\left(t_{o}\right) \times B_{r_{y}}(y) \text {, sendo } y \in \Omega^{\prime},\right.
$$

e

$$
\widehat{u} \text { é constante em }[\tau, 1] \times I_{a}\left(t_{o}\right) \times \Omega^{\prime} .
$$

Usando que $\left(\widehat{x}_{o}, \widehat{f}, \widehat{u}_{r_{y}}\right) \in \mathcal{E}\left(t_{o}, a, \tau, I, \Omega, \Omega^{\prime}, B_{r_{y}}(y)\right)$ e que $\widehat{u}_{r_{y}}(\varepsilon,).\left(\overline{I_{a}\left(t_{o}\right)} \times \overline{B_{r_{y}}(y)}\right) \subset$ $\overline{B_{d}\left(\widehat{x}_{o}(\varepsilon)\right)} \subset \bar{V}$, para todo $y \in \Omega^{\prime}$ concluímos que $\hat{u}$ satisfaz (I). 
Notemos que, dado qualquer $K \subset \subset \Omega^{\prime}$ existem $y_{1}, \ldots, y_{s} \in K$ tais que

$$
K \subset \cup_{i=1}^{s} \overline{B_{\frac{1}{2} r_{y_{i}}}\left(y_{i}\right)} \subset \cup_{i=1}^{s} B_{r_{y_{i}}}\left(y_{i}\right) \subset \Omega^{\prime},
$$

e assim, é fácil verificar que $\widehat{u} \in \mathcal{E}_{M}\left[I_{a}\left(t_{o}\right) \times \Omega^{\prime} ; \mathbb{R}^{n}\right]$.

Seja $u$ a classe de $\widehat{u}$ em $\mathcal{G}\left(I_{a}\left(t_{o}\right) \times \Omega^{\prime} ; \mathbb{R}^{n}\right)$. Então, por (I), temos que $u$ satisfaz de (II) até (IV).

De (I) e 3.2 .7 obtemos (VI) e (VIII). Para as asserções (V) e (VII) basta usar (1) e 3.2 .6 para $\left(\widehat{x}_{o}, \hat{f}, \widehat{u}_{r_{y_{i}}}\right) \in \mathcal{E}\left(t_{o}, a, \tau, I, \Omega, \Omega^{\prime}, B_{r_{y_{i}}}\left(y_{i}\right)\right)$, sendo $1 \leq i \leq n$. //

No próximo resultado além de substituir $W$ por $\Omega^{\prime}$, em 3.2.8, iremos trocar $\Omega$ por $\mathbb{R}^{n}$ e assumir que $x_{o}=0$.

3.2.10 Teorema. Sejam I um intervalo aberto de $\mathbb{R}, t_{0} \in I, \Omega^{\prime}$ um aberto de $\mathbb{R}^{m}$, $f \in \mathcal{G}\left(I \times \mathbb{R}^{n} \times \Omega^{\prime} ; \mathbb{R}^{n}\right)$ e $\hat{f}=\left(\hat{f}_{1}, \ldots, \widehat{f}_{n}\right)$ um representante de $f$. Denotando por $(t, x, y)=\left(t, x_{1}, \ldots, x_{n}, y_{1}, \ldots, y_{m}\right)$ um ponto genérico de $I \times \mathbb{R}^{n} \times \Omega^{\prime}$ tem-se que, se

(i) existem $M>0$ e $\tau \in] 0,1]$ tais que $\widehat{f}(] 0, \tau\left[\times I \times \overline{B_{r}(0)} \times \overline{B_{r}(y)}\right) \subset \overline{B_{M r}(0)}$, para todo $y \in \Omega^{\prime}$ e todo $r>0$ com $\overline{B_{r}(y)} \subset \Omega^{\prime}$, sendo $B_{r}(0)$ e $B_{M r}(0)$ bolas abertas em $\mathbb{R}^{n}$ e $B_{r}(y)$ bola aberta em $\mathbb{R}^{m}$;

(ii) $\frac{\partial \hat{f}_{i}}{\partial x_{j}}$ tem a propriedade (CLL) em $I \times \mathbb{R}^{n} \times \Omega^{\prime}$, para todo $1 \leq i, j \leq n$, então existem $a>0$ com $\overline{I_{a}\left(t_{o}\right)} \subset I$, uma aplicação $u \in \mathcal{G}\left(I_{a}\left(t_{o}\right) \times \Omega^{\prime} ; \mathbb{R}^{n}\right)$ e um representante $\widehat{u}$ de $u$ satisfazendo

(I) $\left(\widehat{x}_{o}, \widehat{f}, \widehat{u}\right)$ satisfaz 3.2.1.vii e 3.2.1.viii(substituindo $W$ por $\Omega^{\prime}$ e $\Omega$ por $\left.\mathbb{R}^{n}\right)$ para algum $\left.\left.\tau_{1} \in\right] 0,1\right]$, onde $\widehat{x}_{o}(\varepsilon)=0$, para todo $\left.\left.\varepsilon \in\right] 0,1\right]$;

(II) $u \in \mathcal{G}_{*}\left(I_{a}\left(t_{o}\right) \times \Omega^{\prime} ; \mathbb{R}^{n}\right)$;

(III) $\frac{\partial u}{\partial t}=f \circ\left(\pi, u, \pi_{1}, \ldots, \pi_{m}\right)$ em $\mathcal{G}\left(I_{a}\left(t_{o}\right) \times \Omega^{\prime} ; \mathbb{R}^{n}\right)$;

$\left.(\mathrm{IV}) u\right|_{\left\{t_{o}\right\} \times \Omega^{\prime}}=0 \quad$ em $\mathcal{G}\left(\Omega^{\prime} ; \mathbb{R}^{n}\right)$;

(V) são verdadeiras as asserçôes $3.2 .9 . \mathrm{V}$ e 3.2.9.VII (substituindo $\Omega$ por $\mathbb{R}^{n}$ ). 
Demonstração. Sejam $M$ e $\tau$ como em (i) e tomemos $a^{*}>0 \operatorname{com} \overline{I_{a^{*}\left(t_{o}\right)}} \subset I$.

Fixemos $y \in \Omega^{\prime}$ e seja $r_{y}>0$ com $\overline{B_{r_{y}}(y)} \subset \Omega^{\prime}$. Então fazendo $K_{1}=\{0\}, V=$ $B_{r_{y}}(0), W=B_{r_{y}}(y), d=r_{y}\left(\right.$ distância de $K_{1}$ a $\left.\mathbb{R}^{n} \backslash V\right)$ e usando que, por (i),

$$
\left.\|\widehat{f}(\varepsilon, t, x, z)\| \leq M r_{y} \text {, para todo }(\varepsilon, t, x, z) \in\right] 0, \tau\left[\times \overline{I_{a}\left(t_{o}\right)} \times \bar{V} \times \bar{W}\right.
$$

temos, analisando as provas de 3.2 .5 e 3.2 .3 , que, se $\left.a<\min \left\{\frac{1}{M}, a^{*}\right\}, \varepsilon \in\right] 0, \tau\left[\right.$ e $T_{y}^{\varepsilon}$ é a aplicação

$$
T_{y}^{\varepsilon}: g \in \mathcal{C}\left(\overline{I_{a}\left(t_{o}\right)} \times \overline{B_{r_{y}}(y)} ; \overline{B_{r_{y}}(0)}\right) \longmapsto T_{y}^{\varepsilon} g \in \mathcal{C}\left(\overline{I_{a}\left(t_{o}\right)} \times \overline{B_{r_{y}}(y)} ; \overline{B_{r_{y}}(0)}\right)
$$

onde

$$
\left(T_{y}^{\varepsilon} g\right)(t, w)=\int_{t_{o}}^{t} \widehat{f}(\varepsilon, s, g(s, w), w) d s,
$$

então existe uma aplicação que será denotada por $u_{r_{y}}$ tal que $u_{r_{y}} \in \mathcal{G}\left(I_{a}\left(t_{o}\right) \times B_{r_{y}}(y) ; \mathbb{R}^{n}\right)$, satisfaz de 3.2.5.II até 3.2.5.IV(substituindo $W$ por $B_{r_{y}}(y)$ e $\Omega$ por $\mathbb{R}^{n}$ ) e ainda existe um representante $\hat{u}_{r_{y}}$ de $u_{r_{y}}$ que está definido em $\left.] 0,1\right] \times \overline{I_{a}\left(t_{o}\right)} \times \overline{B_{r_{y}}(y)}, \widehat{u}_{r_{y}}(\varepsilon,).\left(\overline{I_{a}\left(t_{o}\right)} \times\right.$ $\left.\overline{B_{r_{y}}(y)}\right) \subset \overline{B_{r_{y}}(0)}, \widehat{u}_{r_{y}}(\varepsilon,$.$) é o único ponto fixo de T_{y}^{\varepsilon}$, para todo $\left.\varepsilon \in\right] 0, \tau\left[\right.$ e $\left(\widehat{x}_{o}, \widehat{f}, \widehat{u}_{r_{y}}\right) \in$ $\mathcal{E}\left(t_{o}, a, \tau, I, \mathbb{R}^{n}, \Omega^{\prime}, B_{r_{y}}(y)\right)$.

Notemos que, se $\varepsilon \in] 0, \tau\left[\right.$ e $y, z \in \Omega^{\prime}$ são tais que $K=\overline{B_{r_{y}}(y)} \cap \overline{B_{r_{z}}(z)} \neq \emptyset$ e $T^{\varepsilon}$ é a aplicação, admitindo $r_{y}>r_{z}$,

$$
T^{\varepsilon}: g \in \mathcal{C}\left(\overline{I_{a}\left(t_{o}\right)} \times K ; \overline{B_{r_{y}}(0)}\right) \longmapsto T^{\varepsilon} g \in \mathcal{C}\left(\overline{I_{a}\left(t_{o}\right)} \times K ; \overline{B_{r_{y}}(0)}\right)
$$

onde

$$
\left(T^{\varepsilon} g\right)(t, w)=\int_{t_{o}}^{t} \hat{f}(\varepsilon, s, g(s, w), w) d s
$$

então $T^{\varepsilon}$ tem um único ponto fixo e $\left.\widehat{u}_{r_{y}}(\varepsilon,)\right|_{.\overline{I_{a}\left(t_{o}\right)} \times K}$ e $\left.u_{r_{z}}(\varepsilon,)\right|_{.\overline{I_{a}\left(t_{o}\right)} \times K}$ são pontos fixos de $T^{\varepsilon}$, e assim

$$
\left.u_{r_{y}}(\varepsilon, .)\right|_{\overline{I_{a}\left(t_{o}\right)} \times K}=\left.u_{r_{z}}(\varepsilon, .)\right|_{\overline{I_{a}\left(t_{o}\right)} \times K} .
$$

Portanto podemos definir a aplicação $\widehat{u}$ em $] 0,1] \times I_{a}\left(t_{o}\right) \times \Omega^{\prime}$ por

$$
\left.\widehat{u}=\widehat{u}_{r_{y}} \text { em }\right] 0, \tau\left[\times I_{a}\left(t_{o}\right) \times B_{r_{y}}(y) \text {, sendo } y \in \Omega^{\prime},\right.
$$


e

$\widehat{u}$ é constante em $[\tau, 1] \times I_{a}\left(t_{o}\right) \times \Omega^{\prime}$.

Usando que $\left(\widehat{x}_{o}, \hat{f}, \widehat{u}_{r_{y}}\right) \in \mathcal{E}\left(t_{o}, a, \tau, I, \mathbb{R}^{n}, \Omega^{\prime}, B_{r_{y}}(y)\right)$ para todo $y \in \Omega^{\prime}$, concluímos que $\widehat{u}$ satisfaz (I).

Notemos que se $K \subset \subset \Omega^{\prime}$, então existem $y_{1}, \ldots, y_{s} \in K$ tais que

$$
K \subset \cup_{i=1}^{s} \overline{B_{\frac{1}{2} r_{y_{i}}}\left(y_{i}\right)} \subset \cup_{i=1}^{s} B_{r_{y_{i}}}\left(y_{i}\right) \subset \Omega^{\prime},
$$

e assim é fácil verificar que $\widehat{u} \in \mathcal{E}_{M}\left[I_{a}\left(t_{0}\right) \times \Omega^{\prime} ; \mathbb{R}^{n}\right]$.

De (I) concluímos que a classe de $\widehat{u}$ em $\mathcal{G}\left(I_{a}\left(t_{o}\right) \times \Omega^{\prime} ; \mathbb{R}^{n}\right)$ satisfaz (III) e (IV).

Usando (1) e que $\widehat{u}_{r_{y}}(] 0, \eta\left[\times I_{a}\left(t_{0}\right) \times B_{r_{y}}(y)\right) \subset \overline{B_{r_{y}}(0)}$ para todo $y \in \Omega^{\prime}$, concluímos que $u$ satisfaz (II).

Como $\left(\hat{x}_{o}, \widehat{f}, \widehat{u}_{r_{y}}\right) \in \mathcal{E}\left(t_{o}, a, \tau, I, \mathbb{R}^{n}, \Omega^{\prime}, B_{r_{y}}(y)\right)$ para todo $y \in \Omega^{\prime}$ e vale $(1)$ temos, por 3.2.6 aplicado a $\left(\widehat{x}_{o}, \widehat{f}, \widehat{u}_{r_{y}}\right)$, que a asserção $(\mathrm{V})$ é verdadeira. //

Se em 3.2.10 temos $\Omega^{\prime}=\mathbb{R}^{m}$, então podemos enfraquecer a hipótese 3.2.10.i e obtemos o seguinte resultado :

3.2.11 Teorema. Sejam $I$ um intervalo aberto de $\mathbb{R}, t_{0} \in I, f \in \mathcal{G}\left(I \times \mathbb{R}^{n} \times\right.$ $\left.\mathbb{R}^{m} ; \mathbb{R}^{n}\right)$ e $\hat{f}=\left(\widehat{f}_{1}, \ldots, \widehat{f}_{n}\right)$ um representante de $f$. Denotando por $B_{r}$ (respectivamente $B_{r}^{\prime}$ ) a bola aberta de centro 0 e raio $r$ em $\mathbb{R}^{m}$ (respectivamente $\mathbb{R}^{n}$ ) e por $(t, x, y)=$ $\left(t, x_{1}, \ldots, x_{n}, y_{1}, \ldots, y_{m}\right)$ um ponto genérico de $\mathbb{R}^{n+m+1}$ tem-se que, se

(i) existem $M>0$ e $\tau \in] 0,1]$ tais que $\hat{f}(] 0, \tau\left[\times I \times \overline{B_{r}^{\prime}} \times \overline{B_{r}}\right) \subset \overline{B_{r M}^{\prime}}$, para todo $r>0$;

(ii) $\frac{\partial \hat{f}_{i}}{\partial x_{j}}$ tem a propriedade (CLL) em $I \times \mathbb{R}^{n} \times \mathbb{R}^{m}$, para todo $1 \leq i, j \leq n$,

então existem $a>0$ com $\overline{I_{a}\left(t_{o}\right)} \subset I$, uma aplicação $u \in \mathcal{G}\left(I_{a}\left(t_{o}\right) \times \mathbb{R}^{m} ; \mathbb{R}^{n}\right)$ e um representante $\widehat{u}$ de $u$ tais que

(I) são verdadeiras as asserções 3.2.10.I até 3.2.10.V (substituindo $\Omega^{\prime}$ por $\mathbb{R}^{m}$ e $\Omega$ por $\left.\mathbb{R}^{n}\right)$. 
(II) existe $\left.\left.\eta_{1} \in\right] 0,1\right]$ tal que $\widehat{u}(] 0, \eta_{1}\left[\times I_{a}\left(t_{0}\right) \times \overline{B_{r}}\right) \subset \overline{B_{r}^{\prime}}$, para todo $r>0$;

(III) se existem $M_{1}>0$ e $\left.\left.\tau_{1} \in\right] 0,1\right]$ tais que para todo $1 \leq i, k \leq n$ e $1 \leq j \leq m$ tem-se que

$$
\begin{aligned}
& \frac{\partial \hat{f}_{i}}{\partial x_{k}}(] 0, \tau_{1}\left[\times I \times \mathbb{R}^{n} \times \mathbb{R}^{m}\right) \subset\left[-M_{1} ; M_{1}\right] ; \\
& \frac{\partial \widehat{f}_{i}}{\partial y_{j}}(] 0, \tau_{1}\left[\times I \times \overline{B_{r}^{\prime}} \times \overline{B_{r}}\right) \subset\left[-r M_{1}, r M_{1}\right], \text { para todo } r>0,
\end{aligned}
$$

então existem $M_{2}>0$ e $\tau_{2} \in[0,1]$ tais que para todo $1 \leq i \leq n$ e $1 \leq j \leq m$ tem-se que

$$
\frac{\partial \widehat{u}_{i}}{\partial y_{j}}(] 0, \tau_{1}\left[\times I_{a}\left(t_{o}\right) \times \overline{B_{r}}\right) \subset\left[-r M_{2}, r M_{2}\right] \text {, para todo } r>0 .
$$

Demonstração. Sejam $M$ e $\tau$ como em (i) e tomemos $a^{*}>0 \operatorname{com} \overline{I_{a^{*}}\left(t_{o}\right)} \subset I$ e fixemos $r>0$. Fazendo $K_{1}=\{0\}, V=B_{r}^{\prime}, W=B_{r}, d=r\left(\right.$ distância de $K_{1}^{\prime}$ a $\left.\mathbb{R}^{n} \backslash V\right)$ e usando que, por (i),

$$
\|\hat{f}(\varepsilon, t, x, z)\| \leq M r, \text { para todo }(\varepsilon, t, x, z) \in] 0, \eta\left[\times \overline{I_{a^{*}}\left(t_{o}\right)} \times \bar{V} \times \bar{W}\right.
$$

temos, analisando as provas de 3.2 .5 e 3.2 .3 , que, se $\left.a=\min \left\{\frac{1}{M}, a^{*}\right\}, \varepsilon \in\right] 0, \tau\left[\right.$ e $T_{r}^{\varepsilon}$ é a aplicação

$$
T_{r}^{\varepsilon}: g \in \mathcal{C}\left(\overline{I_{a}\left(t_{o}\right)} \times \overline{B_{r}} ; \overline{B_{r}^{\prime}}\right) \longmapsto T_{r}^{\varepsilon} g \in \mathcal{C}\left(\overline{I_{a}\left(t_{o}\right)} \times \overline{B_{r}} ; \overline{B_{r}^{\prime}}\right)
$$

onde

$$
\left(T_{r}^{\varepsilon} g\right)(t, w)=\int_{t_{o}}^{t} \hat{f}(\varepsilon, s, g(s, w), w) d s,
$$

então existe uma aplicação que será denotada por $u_{r}$ tal que $u_{r} \in \mathcal{G}\left(I_{a}\left(t_{o}\right) \times B_{r} ; \mathbb{R}^{n}\right)$, satisfaz de 3.2.5.II até 3.2.5.IV(substituindo $W$ por $B_{r}$ e $\Omega$ por $\mathbb{R}^{n}$ ) e ainda existe um representante $\widehat{u}_{r}$ de $u_{r}$ que está definido em $\left.] 0,1\right] \times \overline{I_{a}\left(t_{o}\right)} \times \overline{B_{r}}, \widehat{u}_{r}(\varepsilon,).\left(\overline{I_{a}\left(t_{o}\right)} \times \overline{B_{r}}\right) \subset \overline{B_{r}^{\prime}}, \widehat{u}_{r}(\varepsilon,$. é o único ponto fixo de $T_{r}^{\varepsilon}$, para todo $\left.\varepsilon \in\right] 0, \tau\left[\right.$ e $\left(\widehat{x}_{o}, \widehat{f}, \widehat{u}_{r}\right) \in \mathcal{E}\left(t_{o}, a, \tau, I, \mathbb{R}^{n}, \mathbb{R}^{m}, B_{r}\right)$.

Portanto podemos definir a aplicação $\widehat{u} \mathrm{em}] 0,1] \times I_{a}\left(t_{o}\right) \times \mathbb{R}^{m}$ por

$$
\begin{aligned}
& \left.\widehat{u}=\widehat{u}_{r} \text { em }\right] 0, \tau\left[\times I_{a}\left(t_{o}\right) \times B_{r}\right. \\
& \widehat{u} \text { é constante em }[\tau, 1] \times I_{a}\left(t_{o}\right) \times \mathbb{R}^{m} .
\end{aligned}
$$


Com prova análoga à de 3.2 .10 temos que $\widehat{u} \in \mathcal{E}_{M}\left[I_{a}\left(t_{o}\right) \times \mathbb{R}^{m} ; \mathbb{R}^{n}\right]$ e que a asserção (I) é verdadeira, sendo $u$ a classe de $\hat{u}$ em $\mathcal{G}\left(I_{a}\left(t_{o}\right) \times \mathbb{R}^{m} ; \mathbb{R}^{n}\right)$.

Provaremos a seguir (II).

Como $\left.\widehat{u}\right|_{0, \tau\left[\times I_{a}\left(t_{o}\right) \times B_{r}\right.}=\widehat{u}_{r}$, para todo $r>0$ temos que

$$
\widehat{u}(] 0, \tau\left[\times I_{a}\left(t_{o}\right) \times B_{r}\right) \subset \overline{B_{r}^{\prime}}, \quad \text { para todo } r>0 .
$$

Fixemos $r>0$. Como $\overline{B_{r}}=\bigcap_{j \in N} B_{r+\frac{1}{3}}$ e vale (1) temos que, se $(t, y) \in I_{a}\left(t_{o}\right) \times \overline{B_{r}}$, então

$$
\left.\|\widehat{u}(\varepsilon, t, y)\| \leq r+\frac{1}{j}, \quad \text { para todo } j \in \mathbb{N} \text { e } \varepsilon \in\right] 0, \tau[,
$$

e assim $\widehat{u}(\varepsilon, t, y) \in \overline{B_{r}^{\prime}}$, para todo $\left.\varepsilon \in\right] 0, \tau[$. Portanto temos que (II) é verdadeira.

Para terminar provaremos (III).

Usando que $\frac{\partial^{2} \widehat{u}_{i}}{\partial t \partial y_{j}}=\frac{\partial^{2} \widehat{u}_{i}}{\partial y_{j} \partial t}$ para todo $1 \leq i \leq n$ e $1 \leq j \leq m$, e as afirmações 3.2.1.vii e 3.2.1.viii temos que

$$
\frac{\partial \widehat{u}_{i}}{\partial y_{j}}(\varepsilon, t, y)=\int_{t_{\circ}}^{t}\left(\sum_{k=1}^{n} \frac{\partial \widehat{f}_{i}}{\partial x_{k}}(\varepsilon, s, \widehat{u}(\varepsilon, s, y), y) \frac{\partial \widehat{u}_{k}}{\partial y_{j}}(\varepsilon, s, y)+\frac{\partial \widehat{f}_{i}}{\partial y_{j}}(\varepsilon, s, \widehat{u}(\varepsilon, s, y), y)\right) d s,
$$

para todo $(\varepsilon, t, y) \in] 0, \tau\left[\times I_{a}\left(t_{o}\right) \times \mathbb{R}^{m}, \quad 1 \leq i \leq n\right.$ e $1 \leq j \leq m$.

Fixemos $r>0,[c, d] \subset I_{a}\left(t_{o}\right)$ com $t_{o} \in[c, d]$ e $1 \leq j \leq m$.

Sejam $M_{1}$ e $\tau_{1}$ como na hipótese de (III), $\tau_{2}=\min \left\{\tau, \tau_{1}\right\}$ e $\frac{\partial \widehat{u}}{\partial y_{j}}$ a n-upla $\left(\frac{\partial \widehat{u}_{1}}{\partial y_{j}}, \ldots, \frac{\partial \widehat{u}_{n}}{\partial y_{j}}\right)$. Então, usando (2) e (II), temos que

$$
\begin{aligned}
\left\|\frac{\partial \widehat{u}}{\partial y_{j}}(\varepsilon, t, z)\right\| & \leq \sum_{i=1}^{n}\left|\frac{\partial \widehat{u}_{i}}{\partial y_{j}}(\varepsilon, t, z)\right| \\
& \leq\left|\int_{t_{o}}^{t}\left(\sum_{k=1}^{n} M_{1}\left|\frac{\partial \widehat{u}_{k}}{\partial y_{j}}(\varepsilon, s, z)\right|+M_{1} r\right) d s\right| \\
& \leq\left|\int_{t_{o}}^{t}\left(n M_{1}|| \frac{\partial \widehat{u}}{\partial y_{j}}(\varepsilon, s, z) \|+M_{1} r\right) d s\right|
\end{aligned}
$$

para todo $(\varepsilon, t, z) \in] 0, \tau_{2}\left[\times[c, d] \times \overline{B_{r}}\right.$, e assim, pelo Lema de Gronwall (3.1.11), concluímos que, se $\bar{c}=\exp \left(a n M_{1}\right)-1$, então

$$
\left\|\frac{\partial \widehat{u}}{\partial y_{j}}(\varepsilon, t, z)\right\| \leq \frac{M_{1} r}{n M_{1}} \max \left\{\exp \left(n M_{1}\left(d-t_{o}\right)\right)-1, \exp \left(n M_{1}\left(t_{o}-c\right)\right)-1\right\} \leq \frac{r}{n} \bar{c},
$$


para todo $(\varepsilon, t, z) \in] 0, \tau_{2}\left[\times[c, d] \times \overline{B_{r}}\right.$.

Como $c$ e $d$ são arbitrários concluímos que (III) é verdadeira. //

Vamos, a partir daqui, procurar substituir o vetor generalizado $x_{0}$ que aparece em 3.2.5.IV por uma aplicação generalizada.

3.2.12 Teorema(Existência). Sejarı $\Omega$ um aberto de $\mathbb{R}^{n}$, I um intervalo aberto de $\mathbb{R}$, $t_{o} \in I, \Omega^{\prime}$ um aberto de $\mathbb{R}^{m}, W$ um aberto de $\Omega^{\prime}, g \in \mathcal{G}\left(W ; \mathbb{R}^{n}\right), \hat{g}$ um representante de $g, f \in \mathcal{G}\left(I \times \Omega \times \Omega^{\prime} ; \mathbb{R}^{n}\right)$ e $\hat{f}=\left(\hat{f}_{1}, \ldots, \hat{f}_{n}\right)$ um representante de $f$. Denotando por $(t, x, y)=\left(t, x_{1}, \ldots, x_{n}, y_{1}, \ldots, y_{m}\right)$ um ponto genérico de $I \times \Omega \times \Omega^{\prime}$ tem-se que, se

\section{(i) $\bar{W} \subset \subset \Omega^{\prime}$;}

(ii) existem $U$ um aberto de $\Omega$ e $\left.\left.\tau_{1} \in\right] 0,1\right]$ tais que $\bar{U} \subset \subset \Omega$ e $\hat{g}(] 0, \tau_{1}[\times W) \subset U$;

(iii) $g \in \mathcal{G}_{*}(W ; U)$;

(iv) $f \in \mathcal{G}_{*}\left(I \times \Omega \times \Omega^{\prime} ; \mathbb{R}^{n}\right)$;

(v) $\frac{\partial \widehat{f}_{i}}{\partial x_{j}}$ tem a propriedade (CLL) em $I \times \Omega \times \Omega^{\prime}$, para todo $1 \leq i, j \leq n$, então existem $a>0$ com $\left.\left.\overline{I_{a}\left(t_{o}\right)} \subset I, \tau \in\right] 0,1\right], u \in \mathcal{G}\left(I_{a}\left(t_{o}\right) \times W ; \mathbb{R}^{n}\right)$ e um representante $\widehat{u}$ de u satisfazendo:

(I) existe $V$ um aberto de $\Omega$ com $\bar{V} \subset \subset \Omega$ e $\widehat{u}(] 0, \tau\left[\times I_{a}\left(t_{o}\right) \times W\right) \subset V$;

(II) $\frac{\partial \widehat{u}}{\partial t}(\varepsilon, t, y)=\widehat{f}(\varepsilon, t, \widehat{u}(\varepsilon, t, y), y)$, para todo $\left.(\varepsilon, t, y) \in\right] 0, \tau\left[\times I_{a}\left(t_{o}\right) \times W\right.$;

(III) $\widehat{u}\left(\varepsilon, t_{o},.\right)=\hat{g}(\varepsilon,$.$\left.) , para todo \varepsilon \in\right] 0, \tau[$;

(IV) $u \in \mathcal{G}_{*}\left(I_{a}\left(t_{o}\right) \times W ; \Omega\right)$;

(V) $\frac{\partial u}{\partial t}=f \circ\left(\pi, u, \pi_{1}, \ldots, \pi_{m}\right)$ em $\mathcal{G}\left(I_{a}\left(t_{o}\right) \times W ; \mathbb{R}^{n}\right)$;

(VI) $\left.u\right|_{\left\{t_{o}\right\} \times W}=g$ em $\mathcal{G}\left(W ; \mathbb{R}^{n}\right)$;

(VII) se $p \in \mathbb{N}$ e para todo $1 \leq i \leq n$ tem-se que $\partial^{\alpha} f_{i} \in \mathcal{G}_{*}\left(I \times \Omega \times \Omega^{\prime} ; \mathbb{R}\right)$, para todo $\alpha \in N^{n+m+1} \quad$ com $|\alpha| \leq p ;$ 
$\partial^{\gamma} g_{i} \in \mathcal{G}_{*}(W ; \mathbb{R})$, para todo $\gamma \in \mathbb{N}^{m}$ com $|\gamma| \leq p$,

então

$\partial^{\beta} u_{i} \in \mathcal{G}_{*}\left(I_{a}\left(t_{o}\right) \times W ; \mathbb{R}\right)$, para todo $\beta \in \mathbb{N}^{m+1}$ com $|\beta| \leq p \quad e \quad 1 \leq i \leq n ;$

(VIII) se $p \in \mathbb{N}$ e para todo $1 \leq i \leq n$ tem-se que

$\partial^{\alpha} \hat{f}_{i}$ é uma função limitada em $J \times \Omega \times \Omega^{\prime}$, para todo $J \subset \subset I \quad e \quad \alpha \in \mathbb{N}^{n+m+1}$ $\operatorname{com}|\alpha| \leq p$;

$\partial^{\gamma} \widehat{g}_{i}$ é uma função limitada em $W$, para todo $\gamma \in \mathbb{N}^{m}$ com $|\gamma| \leq p$,

então para todo $J_{1} \subset \subset I_{a}\left(t_{o}\right)$ e $\beta \in \mathbb{N}^{m+1}$ com $|\beta| \leq p$ tem-se que

$\partial^{\beta} \widehat{u}_{i}$ é uma função limitada em $J_{1} \times W$, para todo $1 \leq i \leq n$;

(IX) se para todo $1 \leq i, k \leq n$ e $1 \leq j \leq m$ tem-se que

$\frac{\partial f_{i}}{\partial x_{k}}$ e $\frac{\partial f_{i}}{\partial y_{j}} \quad$ pertencem a $\mathcal{G}_{*}\left(I \times \Omega \times \Omega^{\prime} ; \mathbb{R}\right) ;$

$\frac{\partial g_{i}}{\partial y_{j}} \in \mathcal{G}_{*}(W ; \mathbb{R})$

então

$\frac{\partial u_{i}}{\partial y_{j}} \in \mathcal{G}_{*}\left(I_{a}\left(t_{o}\right) \times W ; \mathbb{R}\right)$, para todo $1 \leq i \leq n$ e $1 \leq j \leq m ;$

(X) se para todo $1 \leq i, k \leq n$ e $1 \leq j \leq m$ tem-se que

$\frac{\partial \widehat{f}_{i}}{\partial x_{k}}$ e $\frac{\partial \hat{f}_{i}}{\partial y_{j}} \quad$ são funções limitadas em $J \times \Omega \times \Omega^{\prime}$, para todo $J \subset \subset I$;

$\frac{\partial \widehat{g}_{i}}{\partial y_{j}} \quad$ é uma função limitada em $W$,

então para todo $1 \leq i \leq n$ e $1 \leq j \leq m$ tem-se que

$\frac{\partial \widehat{u}_{i}}{\partial y_{j}} \quad$ é uma função limitada em $J_{1} \times W$, para todo $J_{1} \subset \subset I_{a}\left(t_{o}\right)$.

Demonstração. Sejam $U$ e $\tau_{1}$ como em (ii), e tomemos $U_{1}$ e $U_{2}$ abertos de $\Omega$ tais que $\bar{U} \subset U_{1} \subset \overline{U_{1}} \subset U_{2} \subset \overline{U_{2}} \subset \subset \Omega$ e seja $4 d$ a distância de $\bar{U}_{1}$ a $\Omega \backslash U_{2}$.

Como $\overline{U_{1}} \subset \subset \Omega$ existem $x_{1}, \ldots, x_{r} \in \overline{U_{1}}$ tais que

$$
U_{1} \subset \overline{U_{1}} \subset \cup_{i=1}^{r} B_{d}\left(x_{i}\right) \subset \cup_{i=1}^{r} B_{2 d}\left(x_{i}\right) \subset \Omega \text {. }
$$


Notemos que, se $x \in B_{d}(0)$, então $x+z \in \Omega$ para todo $z \in \overline{U_{1}}$ pois, se $z \in \overline{U_{1}}$ existe $i \in\{1, \ldots, r\}$ tal que $z \in B_{d}\left(x_{i}\right)$, e assim

$$
\left\|x+z-x_{i}\right\| \leq\|x\|+\left\|z-x_{i}\right\|<d+d=2 d,
$$

provando que $x+z \in B_{2 d}\left(x_{i}\right) \subset \Omega$.

Portanto podemos definir a aplicação moderada $\widehat{h}=\left(\widehat{h}_{1}, \ldots, \widehat{h}_{n}\right)$ em $\left.] 0,1\right] \times I \times B_{d}(0) \times$ $U_{1} \times \Omega^{\prime}$ por

$$
\widehat{h}(\varepsilon, s, x, z, y)=\widehat{f}(\varepsilon, s, x+z, y) .
$$

Seja $\widehat{x}_{o}$ a aplicação definida em $\left.] 0,1\right]$ por $\widehat{x}_{o}(\varepsilon)=(0, \ldots, 0) \in \mathbb{R}^{n}$.

Denotaremos por $(s, x, z, y)=\left(s, x_{1}, \ldots, x_{n}, z_{1}, \ldots, z_{n}, y_{1}, \ldots, y_{m}\right)$ um ponto genérico de $I \times B_{d}(0) \times\left(U_{1} \times \Omega^{\prime}\right)$.

Vamos, a seguir, verificar que podemos aplicar $3.2 .5\left(\operatorname{com} \widehat{h}, B_{d}(0), U_{1} \times \Omega^{\prime}\right.$ e $U \times W$ no lugar de $\hat{f}, \Omega, \Omega^{\prime}$ e $W$ respectivamente).

Sejam $J \subset \subset I, K \subset \subset B_{d}(0)$ e $L \subset \subset U_{1} \times \Omega^{\prime}$, e tomemos $K_{2} \subset \subset U_{1}$ e $K^{\prime} \subset \subset \Omega^{\prime}$ tais que $L \subset K_{2} \times K^{\prime}$.

Usando que $K+K_{2} \subset \subset \Omega$ e que valem (iv) e (v), existem $\left.\left.M>0, c>0, \eta \in\right] 0,1\right]$ e $N \in \mathbb{N}$ tais que

$$
\|\widehat{f}(\varepsilon, s, \mu, y)\| \leq M \quad \text { e } \quad\left|\frac{\partial \widehat{f}_{i}}{\partial x_{j}}(\varepsilon, s, \mu, y)\right| \leq \ln \left(c \varepsilon^{-N}\right),
$$

para todo $(\varepsilon, s, \mu, y) \in] 0, \eta\left[\times J \times\left(K+K_{2}\right) \times K^{\prime}\right.$ e $1 \leq i, j \leq n$,

e assim

$$
\|\widehat{h}(\varepsilon, s, x, z, y)\| \leq M \quad \text { e } \quad\left|\frac{\partial \hat{h}_{i}}{\partial x_{j}}(\varepsilon, s, x, z, y)\right| \leq \ln \left(c \varepsilon^{-N}\right),
$$

para todo $(\varepsilon, s, x, z, y) \in] 0, \eta\left[\times J \times K \times\left(K_{2} \times K^{\prime}\right)\right.$ e $1 \leq i, j \leq n$.

Portanto $\hat{h}$ satisfaz 3.2.5.iii e 3.2.5.iv.

Como $\widehat{x}_{o}$ é a aplicação nula é claro que vale 3.2.5.ii, e como $\bar{W} \subset \subset \Omega^{\prime}$ e $\bar{U} \subset \subset U_{1}$, temos que $\bar{U} \times \bar{W} \subset \subset U_{1} \times \Omega^{\prime}$, e portanto vale 3.2.5.i.

Portanto, por 3.2.5, existem $\left.\left.a>0 \operatorname{com} \overline{I_{a}\left(t_{o}\right)} \subset I, \tau_{2} \in\right] 0,1\right]$ e uma aplicação $\hat{v} \in \mathcal{E}_{M}\left[I_{a}\left(t_{o}\right) \times U \times W ; \mathbb{R}^{n}\right]$ tal que $\left(\hat{x}_{o}, \hat{h}, \widehat{v}\right) \in \mathcal{E}\left(t_{o}, a, \tau_{2}, I, B_{d}(0), U_{1} \times \Omega^{\prime}, U \times W\right)($ ver 3.2.1) isto é, existe $V_{1}$ aberto de $B_{d}(0)$ tal que 


$$
\widehat{v}(] 0, \tau_{2}\left[\times I_{a}\left(t_{o}\right) \times U \times W\right) \subset V_{1} \subset \overline{V_{1}} \subset \subset B_{d}(0) ;
$$

$\frac{\partial \widehat{v}}{\partial s}(\varepsilon, s, z, y)=\widehat{h}(\varepsilon, s, \widehat{v}(\varepsilon, s, z, y), z, y)$, para todo $\left.(\varepsilon, s, z, y) \in\right] 0, \tau_{2}\left[\times I_{a}\left(t_{o}\right) \times U \times W\right.$

$$
\left.\widehat{v}\left(\varepsilon, t_{o}, .\right)=\widehat{x}_{o}(\varepsilon)=0, \text { para todo } \varepsilon \in\right] 0, \tau_{2}[\text {. }
$$

Seja $\tau=\min \left\{\tau_{1}, \eta, \tau_{2}\right\}$. Por (ii) podemos definir a aplicação $\widehat{u}=\left(\widehat{u}_{1}, \ldots, \widehat{u}_{n}\right)$ em ] $0,1] \times I_{a}\left(t_{o}\right) \times W$ por

$$
\widehat{u}(\varepsilon, t, y)=\left\{\begin{array}{lc}
\widehat{g}(\varepsilon, y)+\widehat{v}(\varepsilon, t, \widehat{g}(\varepsilon, y), y) & \text { se } \varepsilon \in] 0, \tau[ \\
\widehat{g}\left(\frac{\tau}{2}, y\right)+\widehat{v}\left(\frac{\tau}{2}, t, \widehat{g}\left(\frac{\tau}{2}, y\right), y\right) & \text { se } \varepsilon \in[\tau, 1]
\end{array} .\right.
$$

Usando que $\hat{g} \in \mathcal{E}_{M}\left[W ; \mathbb{R}^{n}\right]$, que $\widehat{v} \in \mathcal{E}_{M}\left[I_{a}\left(t_{o}\right) \times U \times W ; \mathbb{R}^{n}\right]$ e que vale (iii) é fácil verificar que $\hat{u} \in \mathcal{E}_{M}\left[I_{a}\left(t_{o}\right) \times W ; \mathbb{R}^{n}\right]$.

Notemos que, se $V=U+V_{1}$ temos que $V=U+V_{1} \subset U+B_{d}(0) \subset \Omega$, e assim $V$ é um aberto de $\Omega$ com $\bar{V} \subset \bar{U}+\overline{V_{1}} \subset \overline{U_{1}}+B_{d}(0) \subset \Omega$, e portanto, por (ii) e (1), temos que

$$
\widehat{u}(] 0, \tau\left[\times I_{a}\left(t_{o}\right) \times W\right) \subset V \subset \bar{V} \subset \subset \Omega,
$$

e assim temos (I) e (IV), sendo $u$ a classe de $\widehat{u}$ em $\mathcal{G}\left(I_{a}\left(t_{o}\right) \times W ; \mathbb{R}^{n}\right)$.

Usando (2) e (3) temos, para todo $\varepsilon \in] 0, \tau\left[\right.$ e $(t, y) \in I_{a}\left(t_{o}\right) \times W$, que

$$
\begin{aligned}
\frac{\partial \widehat{u}}{\partial t}(\varepsilon, t, y) & =\frac{\partial \widehat{v}}{\partial s}(\varepsilon, t, \widehat{g}(\varepsilon, y), y)=\widehat{h}(\varepsilon, t, \widehat{v}(\varepsilon, t, \widehat{g}(\varepsilon, y), y), \widehat{g}(\varepsilon, y), y) \\
& =\widehat{f}(\varepsilon, t, \widehat{v}(\varepsilon, t, \hat{g}(\varepsilon, y), y)+\widehat{g}(\varepsilon, y), y) \\
& =\widehat{f}(\varepsilon, t, \widehat{u}(\varepsilon, t, y), y) ; \\
\widehat{u}\left(\varepsilon, t_{o}, .\right) & =\widehat{g}(\varepsilon, .)+\widehat{v}\left(\varepsilon, t_{o}, \widehat{g}(\varepsilon, .), .\right)=\widehat{g}(\varepsilon, .)+\widehat{x}_{o}(\varepsilon)=\widehat{g}(\varepsilon, .),
\end{aligned}
$$

e portanto as afirmações (II) e (III) são verdadeiras.

De (II) concluímos (V) e a asserção (VI) é uma conseqüência de (III).

Seja $v$ a classe de $\hat{v}$ em $\mathcal{G}\left(I_{a}\left(t_{o}\right) \times U \times W ; \mathbb{R}^{n}\right)$. Então usando $3.2 .8\left(\operatorname{com} \hat{h}, B_{d}(0)\right.$, $U_{1} \times \Omega^{\prime}, U \times W, \widehat{v}$ e $v$ no lugar de $\hat{f}, \Omega, \Omega^{\prime}, W, \widehat{u}$ e $u$ respectivamente ) temos informações sobre $\partial^{\mu} v_{i}$, sendo $\mu \in \mathbb{N}^{n+m+1}$ e $1 \leq i \leq n$. Usando estas informações e 
que dados quaisquer $(\varepsilon, t, y) \in] 0, \tau\left[\times I_{a}\left(t_{o}\right) \times W, 1 \leq i \leq n\right.$ e $\beta \in \mathbb{N}^{m+1}$ tem-se que $\partial^{\beta} \widehat{u}_{i}(\varepsilon, t, y)$ é soma de produtos de elementos de $A_{1} \cup B_{1}$, sendo

$$
A_{1}=\left\{\partial^{\mu} \widehat{v}_{i}(\varepsilon, t, \widehat{g}(\varepsilon, y), y): \mu \in \mathbb{N}^{n+m+1} \operatorname{com}|\mu| \leq|\beta|\right\}
$$

e

$$
B_{1}=\{1\} \cup\left\{\prod_{(\lambda, j) \in \Lambda \times A} \partial^{\lambda} \widehat{g}_{i}(\varepsilon, y): \Lambda \times A \subset\left\{\gamma \in \mathbb{N}^{m}:|\gamma| \leq|\beta|\right\} \times\{1, \ldots, n\}\right\},
$$

concluímos as outras asserções. //

O Teorema acima forneceu, sob certas condições, uma solução para a equação 3.2.12.V, com a condição 3.2.12.VI, em $\mathcal{G}\left(I_{a}\left(t_{o}\right) \times W ; \mathbb{R}^{n}\right)$ sendo $\bar{W} \subset \subset \Omega^{\prime}$. Iremos, a seguir, procurar substituir $W$ por $\Omega^{\prime}$. Estes resultados serão usados na próxima seção.

3.2.13 Teorema. Sejam $\Omega$ um aberto de $\mathbb{R}^{n}, I$ um intervalo aberto de $\mathbb{R}, t_{o} \in I$, $\Omega^{\prime}$ um aberto de $\mathbb{R}^{m}, g=\left(g_{1}, \ldots, g_{n}\right) \in \mathcal{G}\left(\Omega^{\prime} ; \mathbb{R}^{n}\right), f \in \mathcal{G}\left(I \times \Omega \times \Omega^{\prime} ; \mathbb{R}^{n}\right)$, $\hat{g}$ um representante de $g$ e $\hat{f}=\left(\widehat{f}_{1}, \ldots, \widehat{f}_{n}\right)$ um representante de $f$. Denotando por $(t, x, y)=$ $\left(t, x_{1}, \ldots, x_{n}, y_{1}, \ldots, y_{m}\right)$ um ponto genérico de $I \times \Omega \times \Omega^{\prime}$ tem-se que, se

(i) existem $U$ aberto de $\Omega$ e $\left.\left.\tau_{1} \in\right] 0,1\right]$ tais que $\bar{U} \subset \subset \Omega$ e $\widehat{g}(] 0, \tau_{1}\left[\times \Omega^{\prime}\right) \subset U$;

(ii) $g \in \mathcal{G}_{*}\left(\Omega^{\prime} ; U\right)$;

(iii) $\hat{f}$ é uma aplicaçấo limitada em $I \times \Omega \times \Omega^{\prime}$;

(iv) $\frac{\partial \widehat{f}_{i}}{\partial x_{j}}$ tem a propriedade (CLL) em $I \times \Omega \times \Omega^{\prime}$, para todo $1 \leq i, j \leq n$,

então existem $a>0$ com $\left.\left.\overline{I_{a}\left(t_{o}\right)} \subset I, \tau \in\right] 0,1\right], u \in \mathcal{G}\left(I_{a}\left(t_{o}\right) \times \Omega^{\prime} ; \mathbb{R}^{n}\right)$ e um representante $\widehat{u}$ de u satisfazendo:

(I) existe $V$ um aberto de $\Omega$ com $\bar{V} \subset \subset \Omega$ e $\widehat{u}(] 0, \tau\left[\times I_{a}\left(t_{o}\right) \times \Omega^{\prime}\right) \subset V$;

(II) $\frac{\partial \widehat{u}}{\partial t}(\varepsilon, t, y)=\widehat{f}(\varepsilon, t, \widehat{u}(\varepsilon, t, y), y)$, para todo $\left.(\varepsilon, t, y) \in\right] 0, \tau\left[\times I_{a}\left(t_{o}\right) \times \Omega^{\prime}\right.$;

(III) $\widehat{u}\left(\varepsilon, t_{o},.\right)=\widehat{g}(\varepsilon,$.$\left.) , para todo \varepsilon \in\right] 0, \tau[$;

(IV) $u \in \mathcal{G}_{*}\left(I_{a}\left(t_{o}\right) \times \Omega^{\prime} ; \Omega\right)$; 
(V) $\frac{\partial u}{\partial t}=f \circ\left(\pi, u, \pi_{1}, \ldots, \pi_{m}\right)$ em $\mathcal{G}\left(I_{a}\left(t_{0}\right) \times \Omega^{\prime} ; \mathbb{R}^{n}\right)$;

(VI) $\left.u\right|_{\left\{t_{o}\right\} \times \Omega^{\prime}}=g$ em $\mathcal{G}\left(\Omega^{\prime} ; \mathbb{R}^{n}\right)$;

(VII) se $p \in \mathbb{N}$ e para todo $1 \leq i \leq n$ tem-se que

$\partial^{\alpha} f_{i} \in \mathcal{G}_{*}\left(I \times \Omega \times \Omega^{\prime} ; \mathbb{R}\right)$, para todo $\alpha \in \mathbb{N}^{n+m+1}$ com $|\alpha| \leq p$;

$\partial^{\gamma} g_{i} \in \mathcal{G}_{*}\left(\Omega^{\prime} ; \mathbb{R}\right)$, para todo $\gamma \in \mathbb{N}^{m}$ com $|\gamma| \leq p$

então

$\partial^{\beta} u_{i} \in \mathcal{G}_{*}\left(I_{a}\left(t_{o}\right) \times \Omega^{\prime} ; \mathbb{R}\right)$, para todo $\beta \in \mathbb{N}^{m+1}$ com $|\beta| \leq p$ e $1 \leq i \leq n ;$

(VIII) se $p \in \mathbb{N}$ e para todo $1 \leq i \leq n$ tem-se que

$\partial^{\alpha} \hat{f}_{i}$ é uma função limitada em $J \times \Omega \times \Omega^{\prime}$, para todo $J \subset \subset I$ e $\alpha \in \mathbb{N}^{n+m+1}$ com $|\alpha| \leq p ;$

$\partial^{\gamma} \widehat{g}_{i} \quad$ é uma função limitada em $\Omega^{\prime}$, para todo $\gamma \in \mathbb{N}^{m}$ com $|\gamma| \leq p$, então para todo $J_{1} \subset \subset I_{a}\left(t_{o}\right)$ e $\beta \in \mathbb{N}^{m+1}$ com $|\beta| \leq p$ tem-se que

$\partial^{\beta} \widehat{u}_{i}$ é uma função limitada em $J \times \Omega^{\prime}$, para todo $1 \leq i \leq n$;

(IX) se para todo $1 \leq i, k \leq n$ e $1 \leq j \leq m$ tem-se que

$\frac{\partial f_{i}}{\partial x_{k}}$ e $\frac{\partial f_{i}}{\partial y_{j}} \quad$ pertencem a $\mathcal{G}_{*}\left(I \times \Omega \times \Omega^{\prime} ; \mathbb{R}\right) ;$

$\frac{\partial g_{i}}{\partial y_{j}} \in \mathcal{G}_{*}\left(\Omega^{\prime} ; \mathbb{R}\right)$,

então

$\frac{\partial u_{i}}{\partial y_{j}} \in \mathcal{G}_{*}\left(I_{a}\left(t_{o}\right) \times \Omega^{\prime} ; \mathbb{R}\right)$, para todo $1 \leq i \leq n$ e $1 \leq j \leq m ;$

(X) se para todo $1 \leq i, k \leq n$ e $1 \leq j \leq m$ tem-se que

$\frac{\partial \hat{f}_{i}}{\partial x_{k}}$ e $\frac{\partial \hat{f}_{i}}{\partial y_{j}} \quad$ são funções limitadas em $J \times \Omega \times \Omega^{\prime}$, para todo $J \subset \subset I$;

$\frac{\partial \widehat{g}_{i}}{\partial y_{j}}$ é uma função limitada em $\Omega^{\prime}$,

então para todo $J \subset \subset I_{a}\left(t_{o}\right)$ tem-se que 
$\frac{\partial \widehat{u}_{i}}{\partial y_{j}} \quad$ é uma função limitada em $J \times \Omega^{\prime}$, para todo $1 \leq i \leq n$ e $1 \leq j \leq m$.

Demonstração. Basta repetir a prova de 3.2 .12 (substituindo $W$ por $\Omega^{\prime}$ ), observar que (iii) garante que $f \in \mathcal{G}_{*}\left(I \times \Omega \times \Omega^{\prime} ; \mathbb{R}^{n}\right)$, e usar 3.2 .9 no lugar de 3.2 .5 e de 3.2 .8 . //

Quando $\Omega=\mathbb{R}^{n}$ podemos substituir 3.2.13.i e 3.2.13.ii por 3.2.14.i.

3.2.14 Teorema. Sejam I um intervalo aberto de $\mathbb{R}, t_{0} \in I, \Omega^{\prime}$ um aberto de $\mathbb{R}^{m}$, $g \in \mathcal{G}\left(\Omega^{\prime} ; \mathbb{R}^{n}\right), f \in \mathcal{G}\left(I \times \mathbb{R}^{n} \times \Omega^{\prime} ; \mathbb{R}^{n}\right), \widehat{g}$ um representante de $g$ e $\hat{f}=\left(\hat{f}_{1}, \ldots, \hat{f}_{n}\right)$ um representante de $f$. Denotando por $(t, x, y)=\left(t, x_{1}, \ldots, x_{n}, y_{1}, \ldots, y_{m}\right)$ um ponto genérico de $I \times \mathbb{R}^{n} \times \Omega^{\prime}$ tem-se que, se

(i) $g \in \mathcal{G}_{*}\left(\Omega^{\prime} ; \mathbb{R}^{n}\right)$;

(ii) $\widehat{f}$ é uma aplicação limitada $\mathrm{em} I \times \mathbb{R}^{n} \times \Omega^{\prime}$;

(iii) $\frac{\partial \widehat{f}_{i}}{\partial x_{j}}$ tem a propriedade (CLL) em $I \times \mathbb{R}^{n} \times \Omega^{\prime}$, para todo $1 \leq i, j \leq n$, então existem $a>0$ com $\overline{I_{a}\left(t_{o}\right)} \subset I$ e $u \in \mathcal{G}\left(I_{a}\left(t_{o}\right) \times \Omega^{\prime} ; \mathbb{R}^{n}\right)$ e um representante $\hat{u}$ de $u$ tais que, substituindo $\Omega$ por $\mathbb{R}^{n}$ (quando for o caso), as asserções 3.2.13.II até 3.2.13.X são verdadeiras.

Demonstração. Análoga à de 3.2 .12 observando que, neste caso, a aplicação $\widehat{h}$ pode ser definida em $] 0,1] \times I \times \mathbb{R}^{n} \times \mathbb{R}^{n} \times \Omega^{\prime}$, e usar 3.2 .9 no lugar de 3.2 .5 e de 3.2.8. //

Quando $\Omega=\mathbb{R}^{n}$ e $\Omega^{\prime}=\mathbb{R}^{m}$ obtemos o seguinte resultado:

3.2.15 Teorema. Sejam I um intervalo aberto de $\mathbb{R}, t_{o} \in I, g \in \mathcal{G}\left(\mathbb{R}^{m} ; \mathbb{R}^{n}\right), f \in$ $\mathcal{G}\left(I \times \mathbb{R}^{n} \times \mathbb{R}^{m} ; \mathbb{R}^{n}\right)$, $\hat{g}$ um representante de $g$ e $\hat{f}=\left(\hat{f}_{1}, \ldots, \hat{f}_{n}\right)$ um representante de $f$. Denotando por $B_{r}$ (respectivamente $B_{r}^{\prime}$ ) a bola aberta de centro 0 e raio $r$ em $\mathbb{R}^{m}$ (respectivamente $\left.\mathbb{R}^{n}\right)$ e por $(t, x, y)=\left(t, x_{1}, \ldots, x_{n}, y_{1}, \ldots, y_{m}\right)$ um ponto genérico de $I \times \mathbb{R}^{n} \times \mathbb{R}^{m}$ tem-se que, se 
(i) $g \in \mathcal{G}_{*}\left(\mathbb{R}^{m} ; \mathbb{R}^{n}\right)$;

(ii) existem $M>0$ e $\tau \in] 0,1]$ tais que $\hat{f}(] 0, \tau\left[\times I \times \overline{B_{r}^{\prime}} \times \overline{B_{r}}\right) \subset \overline{B_{M r}^{\prime}}$, para todo $r>0$;

(iii) $\frac{\partial \hat{f}_{i}}{\partial x_{j}}$ tem a propriedade (CLL) em $I \times \mathbb{R}^{n} \times \mathbb{R}^{m}$, para todo $1 \leq i, j \leq n$,

então existem $a>0$ com $\overline{I_{a}\left(t_{o}\right)} \subset I$ e $u \in \mathcal{G}\left(I_{a}\left(t_{o}\right) \times \mathbb{R}^{m} ; \mathbb{R}^{n}\right)$ e um representante $\widehat{u}$ de $u$ tais que

(I) substituindo $\Omega$ por $\mathbb{R}^{n}$ (quando for o caso), as asserções 3.2.13.II até 3.2.13.VII e 3.2.13.IX são verdadeiras;

(II) se existem $\tau \in] 0,1]$ e $M>0$ tais que $\widehat{g}(] 0, \tau\left[\times \overline{B_{r}}\right) \subset \overline{B_{r M}^{\prime}}, \quad$ para todo $r>0$, então existem $M_{1}>0$ e $\left.\left.\tau_{1} \in\right] 0,1\right]$ tais que $\widehat{u}(] 0, \tau_{1}\left[\times I_{a}\left(t_{0}\right) \times \overline{B_{r}}\right) \subset \overline{B_{r M_{1}}^{\prime}}$, para todo $r>0 ;$

(III) se existem $M_{2}>0$ e $\left.\left.\tau_{2} \in\right] 0,1\right]$ tais que para todo $1 \leq i, k \leq n$ e $1 \leq j \leq m$ tem-se que

$$
\begin{aligned}
& \frac{\partial \widehat{f}_{i}}{\partial x_{k}}(] 0, \tau_{2}\left[\times I \times \mathbb{R}^{n} \times \mathbb{R}^{m}\right) \subset\left[-M_{2}, M_{2}\right] ; \\
& \frac{\partial \widehat{f}_{i}}{\partial y_{j}}(] 0, \tau_{2}\left[\times I \times \overline{B_{r}^{\prime}} \times \overline{B_{r}}\right) \subset\left[-r M_{2}, r M_{2}\right], \text { para todo } r>0 ; \\
& \frac{\partial \hat{g}_{i}}{\partial y_{j}}(] 0, \tau_{2}\left[\times \mathbb{R}^{m}\right) \subset\left[-M_{2}, M_{2}\right] ; \\
& \widehat{g}(] 0, \tau_{2}\left[\times \overline{B_{r}}\right) \subset \overline{B_{r M_{2}}^{\prime}}, \quad \text { para todo } r>0,
\end{aligned}
$$

então existem $\bar{M}_{3}>0$ e $\left.\left.\tau_{3} \in\right] 0,1\right]$ tais que para todo $1 \leq i \leq n$ e $1 \leq j \leq m$ tem-se que

$$
\frac{\partial \widehat{u}_{i}}{\partial y_{j}}(] 0, \tau_{3}\left[\times I_{a}\left(t_{o}\right) \times \overline{B_{r}}\right) \subset\left[-r M_{3}, r M_{3}\right], \text { para todo } r>M_{2} .
$$

Demonstração. Análoga à de 3.2.12 observando que, neste caso, a aplicação $\widehat{h}$ está definida em $] 0,1] \times I \times \mathbb{R}^{n} \times\left(\mathbb{R}^{n} \times \mathbb{R}^{m}\right)$, substituindo 3.2 .5 e 3.2 .8 por 3.2 .11 e usando que para 
todo $\tau \in] 0,1]$ e $r>0$ tem-se que, se $B_{r}^{\prime \prime}$ denota a bola aberta de centro 0 e raio $r$ em $\mathbb{R}^{n+m}$, então $B_{r}^{\prime \prime} \subset B_{r}^{\prime} \times B_{r}$ e

$$
\begin{aligned}
\hat{h}(] 0, \tau\left[\times I \times \overline{B_{r}^{\prime}} \times \overline{B_{r}^{\prime \prime}}\right) & \subset \hat{f}(] 0, \tau\left[\times I \times\left(\overline{B_{r}^{\prime}}+\overline{B_{r}^{\prime}}\right) \times \overline{B_{r}}\right) \\
& \subset \hat{f}(] 0, \tau\left[\times I \times \overline{B_{2 r}^{\prime}} \times \overline{B_{r}}\right) \\
& \subset \hat{f}(] 0, \tau\left[\times I \times \overline{B_{2 r}^{\prime}} \times \overline{B_{2 r}}\right) \subset \overline{B_{2 r M}^{\prime}} \cdot / /
\end{aligned}
$$

Completamos os dois resultados anteriores com o seguinte exemplo:

3.2.16 Exemplo. Sejam I um intervalo aberto de $\mathbb{R}, \Omega^{\prime}$ um aberto de $\mathbb{R}^{m}, 1 \leq i \leq n$, $\psi_{i} \in \mathcal{C}^{\infty}(\mathbb{R} ; \mathbb{R}), \Psi_{i} \in \mathcal{C}^{\infty}(\mathbb{R} ; \mathbb{R}), \varphi_{i} \in \mathcal{C}^{\infty}\left(I \times \mathbb{R}^{n} \times \Omega^{\prime} ; \mathbb{R}\right)$ e $\mu_{i}$ uma funçâo definida em [0,1] e com valores em $\mathbb{R}$ tais que $\mu_{i}, \psi_{i}, \Psi_{i}$ e todas as derivadas de $\Psi_{i}$ são funções limitadas. Se $\hat{f}=\left(\widehat{f}_{1}, \ldots, \hat{f}_{n}\right)$ é a aplicação moderada definida em $\left.] 0,1\right] \times I \times \mathbb{R}^{n} \times \Omega^{\prime}$, onde $\widehat{f}_{i}$ é uma das funções

$$
\hat{l}_{1}(\varepsilon, t, x, y)=\psi_{i}\left(\mu_{i}(\varepsilon) \varphi_{i}(t, x, y)\right) \quad e \quad \hat{l}_{2}(\varepsilon, t, x, y)=\Psi_{i}\left(\ln \left(\frac{1}{\varepsilon}\right) \varphi_{i}(t, x, y)\right),
$$

para todo $1 \leq i \leq n$, então $\hat{f}$ satisfaz 3.2.14.ii e 3.2.14.iii..

Se $\Omega=\mathbb{R}^{n}, \Omega^{\prime}=\mathbb{R}^{m}$, $\nu_{i}$ é uma função definida em ]0,1] e com valores em $\mathbb{R}$ limitada, para todo $1 \leq i \leq n e(t, x, p)=\left(t, x_{1}, \ldots, x_{n}, y_{1}, \ldots, y_{m}\right)$ denota um ponto genérico de $\mathbb{R}^{n+m+1}$, então a aplicação moderada, $\hat{f}=\left(\hat{f}_{1}, \ldots, \hat{f}_{n}\right)$, definida em $\left.] 0,1\right] \times \mathbb{R}^{n} \times \mathbb{R}^{m}$, onde $\widehat{f}_{i}$, para todo $1 \leq i \leq n$, é uma das funções

$$
\begin{aligned}
& \hat{l}_{3}\left(\varepsilon, t, x_{1}, \ldots, x_{n}, y_{1}, \ldots, y_{m}\right)=\hat{l}_{1}(\varepsilon, t, x, y) \nu_{i}(\varepsilon) x_{i} \\
& l_{4}\left(\varepsilon, t, x_{1}, \ldots, x_{n}, y_{1}, \ldots, y_{m}\right)=\widehat{l}_{2}(\varepsilon, t, x, y) \nu_{i}(\varepsilon) x_{i} \\
& \hat{l}_{5}\left(\varepsilon, t, x_{1}, \ldots, x_{n}, y_{1}, \ldots, y_{m}\right)=\nu_{i}(\varepsilon) x_{i}+\mu_{i}(\varepsilon) y_{i}
\end{aligned}
$$

satisfaz 3.2.15.ii e 3.2.15.iii. 
A seguir apresentamos um Teorema de Unicidade para soluções da equação 3.2.13.V com a condição 3.2.13.VI.

3.2.17 Teorema (Unicidade). Sejam $\Omega$ um aberto de $\mathbb{R}^{n}$. I um intervalo aberto de $\mathbb{R}$, $t_{0} \in I, \Omega^{\prime}$ um aberto de $\mathbb{R}^{m}, f \in \mathcal{G}\left(I \times \Omega \times \Omega^{\prime} ; \mathbb{R}^{n}\right)$ e $\hat{f}$ um representante de $f$. Denotando por $(t, x, y)=\left(t, x_{1}, \ldots, x_{n}, y_{1}, \ldots, y_{m}\right)$ um ponto genérico de $I \times \Omega \times \Omega^{\prime}$ tem-se que, se

(i) $\hat{f}$ tem a propriedade (LLL) em $\left(I, \Omega, \Omega^{\prime}\right)$;

(ii) $\frac{\partial \widehat{f}_{i}}{\partial x_{j}}$ tem a propriedade (CLL) em $I \times \Omega \times \Omega^{\prime}$, para todo $1 \leq i, j \leq n$, e se existem $u$ e $v$ pertencentes a $\mathcal{G}_{*}\left(I \times \Omega^{\prime} ; \Omega\right)$ tais que

(iii) $\frac{\partial u}{\partial t}=f \circ\left(\pi, u, \pi_{1}, \ldots, \pi_{m}\right)$;

(iv) $\frac{\partial v}{\partial t}=f \circ\left(\pi, v, \pi_{1}, \ldots, \pi_{m}\right)$;

(v) $\left.u\right|_{\left\{t_{o}\right\} \times \Omega^{\prime}}=\left.v\right|_{\left\{t_{o}\right\} \times \Omega^{\prime}}$,

então $u=v$.

Demonstração. Sejam $\left(J_{j}\right)_{j \in N}$ uma seqüência exaustiva de compactos para $I$ com $t_{o} \in$ $\cap_{j \in N} J_{j}$ e $J_{j}$ intervalo fechado para todo $j \in \mathbb{N},\left(K_{j}^{\prime}\right)_{j \in N}$ uma seqüência exaustiva de compactos para $\Omega^{\prime}, \widehat{u}$ um representante de $u$ e $\widehat{v}$ um representante de $v$.

Provaremos que $\left.(\widehat{v}-\widehat{u})\right|_{\underbrace{}_{j} \times K_{j}^{\prime}} \in \mathcal{N}\left[\stackrel{\circ}{J}_{j} \times \stackrel{\circ}{K_{j}^{\prime}} ; \mathbb{R}^{n}\right]$, para todo $j \in \mathbb{N}$, e assim $u=v$

Fixemos $j \in \mathbb{N}$.

Como $u \in \mathcal{G}_{*}\left(I \times \Omega^{\prime} ; \Omega\right), v \in \mathcal{G}_{*}\left(I \times \Omega^{\prime} ; \Omega\right), J_{j} \subset \subset I$ e $K_{j}^{\prime} \subset \subset \Omega^{\prime}$ existem $K_{j} \subset \subset \Omega$ e $\left.\left.\eta_{j} \in\right] 0,1\right]$ tais que

$$
\widehat{u}(] 0, \eta_{j}\left[\times J_{j} \times K_{j}^{\prime}\right) \cup \widehat{v}(] 0, \eta_{j}\left[\times J_{j} \times K_{j}^{\prime}\right) \subset K_{j} \subset \subset \Omega .
$$

Usando (iii), (iv), (v), (1) e 1.1.21.II existem $\hat{g}=\left(\widehat{g}_{1}, \ldots, \widehat{g}_{n}\right) \in \mathcal{N}\left[\stackrel{\circ}{J_{j}} \times \stackrel{\circ}{K_{j}^{\prime}} ; \mathbb{R}^{n}\right]$ e $\hat{h}=\left(\hat{h}_{1}, \ldots \hat{h}_{n}\right) \in \mathcal{N}\left[\stackrel{\circ}{K_{j}^{\prime}} ; \mathbb{R}^{n}\right]$ tais que 


$$
\widehat{g}(\varepsilon, t, y)=\frac{\partial \widehat{v}}{\partial t}(\varepsilon, t, y)-\widehat{f}(\varepsilon, t, \widehat{v}(\varepsilon, t, y), y)-\frac{\partial \widehat{u}}{\partial t}(\varepsilon, t, y)+\widehat{f}(\varepsilon, t, \widehat{u}(\varepsilon, t, y), y)
$$

$\mathrm{e}$

$$
\widehat{h}(\varepsilon, y)=\widehat{v}\left(\varepsilon, t_{o}, y\right)-\widehat{u}\left(\varepsilon, t_{o}, y\right)
$$

para todo $(t, y) \in \stackrel{\circ}{J}_{j} \times \stackrel{\circ}{K}_{j}^{\prime} \quad$ e $\left.\quad \varepsilon \in\right] 0, \eta_{j}[$.

Portanto, para todo $(t, y) \in \stackrel{\circ}{J}_{j} \times \stackrel{\circ}{K}_{j}^{\prime}$ e $\left.\varepsilon \in\right] 0, \eta_{j}[$, temos que

$$
(\widehat{\imath}-\widehat{u})(\varepsilon, t, y)=\widehat{h}(\varepsilon, y)+\int_{t_{o}}^{t}(\widehat{f}(\varepsilon, s, \widehat{v}(\varepsilon, s, y), y)-\widehat{f}(\varepsilon, s, \widehat{u}(\varepsilon, s, y), y)+\widehat{g}(\varepsilon, s, y)) d s,
$$

e assim se $l$ é a aplicação definida em $] 0,1] \times \stackrel{\circ}{J}_{j} \times \stackrel{\circ}{K}_{j}^{\prime}$ por

$$
l(\varepsilon, t, y)=\widehat{v}(\varepsilon, t, y)-\widehat{u}(\varepsilon, t, y)-\widehat{h}(\varepsilon, y)
$$

temos que

$$
l(\varepsilon, t, y)=\int_{t_{o}}^{t}(\widehat{f}(\varepsilon, s, \widehat{v}(\varepsilon, s, y), y)-\widehat{f}(\varepsilon, s, \widehat{u}(\varepsilon, s, y), y)+\widehat{g}(\varepsilon, s, y)) d s .
$$

Sejam $J \subset \subset \stackrel{\circ}{J}_{j}, K^{\prime} \subset \subset \stackrel{\circ}{K_{j}^{\prime}}$ e $\beta \in \mathbb{N}^{m+1}$. Provaremos que, dado $q \in \mathbb{N}$ existem $c>0$ e $\eta \in] 0,1]$ tais que $\left|\partial^{\beta} \widehat{v}_{i}(\varepsilon, t, y)-\partial^{\beta} \widehat{u}_{i}(\varepsilon, t, y)\right| \leq c \varepsilon^{q}$, para todo $\left.(\varepsilon, t, y) \in\right] 0, \eta\left[\times J \times K^{\prime}\right.$ e $1 \leq i \leq n$.

Provaremos (4) usando o Princípio de Indução Finita sobre $|\beta|$. Antes porém faremos algumas considerações.

Seja $q \in \mathbb{I N}$ e tomemos $a$ e $b$ números reais tais que $J \cup\left\{t_{o}\right\} \subset[a, b] \subset \subset \stackrel{\circ}{J}_{j}$.

Como $\hat{f}=\left(\widehat{f}_{1}, \ldots, \hat{f}_{n}\right) \in \mathcal{E}_{M}\left[I \times \Omega \times \Omega^{\prime} ; \mathbb{R}^{n}\right]$ e vale (i) e (ii) podemos encontrar $N \in \mathbb{N}$, $\bar{c}>0$ e $\tau \in] 0, \eta_{j}[$ tais que

$$
\begin{gathered}
\left|\frac{\partial \widehat{f}_{i}}{\partial x_{j}}(\varepsilon, t, x, y)\right| \leq \ln \left(\bar{c} \varepsilon^{-N}\right) \quad, \quad\left|\widehat{f}_{i}(\varepsilon, t, x, y)-\widehat{f}_{i}(\varepsilon, t, z, y)\right| \leq \ln \left(\bar{c} \varepsilon^{-N}\right)\|x-z\| \\
\left|\partial^{\alpha} \widehat{f}_{i}(\varepsilon, t, x, y)\right| \leq \bar{c} \varepsilon^{-N} \quad \text { e }\left|\partial^{\alpha} \widehat{f}_{i}(\varepsilon, t, x, y)-\partial^{\alpha} \widehat{f}_{i}(\varepsilon, t, z, y)\right| \leq \bar{c} \varepsilon^{-N}\|x-z\|
\end{gathered}
$$

para todo $\varepsilon \in] 0, \tau\left[,(t, x, y, z) \in[a, b] \times K_{j} \times K^{\prime} \times K_{j}, \alpha \in \mathbb{N}^{n+m+1} \operatorname{com}|\alpha| \leq|\beta| \mathrm{e}\right.$ $1 \leq i, j \leq n$.

Portanto, usando (1), temos que 


$$
\begin{gathered}
\left|\frac{\partial \widehat{f}_{i}}{\partial x_{j}}(\varepsilon, t, \widehat{u}(\varepsilon, t, y), y)\right| \leq \ln \left(\bar{c} \varepsilon^{-N}\right) ; \quad\left|\frac{\partial \widehat{f}_{i}}{\partial x_{j}}(\varepsilon, t, \widehat{v}(\varepsilon, t, y), y)\right| \leq \ln \left(\bar{c} \varepsilon^{-N}\right), \\
\left|\widehat{f}_{i}(\varepsilon, t, \widehat{u}(\varepsilon, t, y), y)-\widehat{f}_{i}(\varepsilon, t, \widehat{v}(\varepsilon, t, y), y)\right| \leq \ln \left(\bar{c} \varepsilon^{-N}\right)|| \widehat{u}(\varepsilon, t, y)-\widehat{v}(\varepsilon, t, y)|| ; \\
\left|\partial^{\alpha} \widehat{f}_{i}(\varepsilon, t, \widehat{u}(\varepsilon, t, y), y)\right| \leq \bar{c} \varepsilon^{-N} ; \quad\left|\partial^{\alpha} \widehat{f}_{i}(\varepsilon, t, \widehat{v}(\varepsilon, t, y), y)\right| \leq \bar{c} \varepsilon^{-N}
\end{gathered}
$$

$\left|\partial^{\alpha} \hat{f}_{i}(\varepsilon, t, \widehat{u}(\varepsilon, t, y), y)-\partial^{\alpha} \hat{f}_{i}(\varepsilon, t, \widehat{v}(\varepsilon, t, y), y)\right| \leq \bar{c} \varepsilon^{-N}\|\widehat{u}(\varepsilon, t, y)-\widehat{v}(\varepsilon, t, y)\|$,

para todo $\varepsilon \in] 0, \tau\left[,(t, y) \in[a, \ell] \times K^{\prime}, \alpha \in \mathbb{N}^{n+m+1} \operatorname{com}|\alpha| \leq|\beta|\right.$ e $1 \leq i \leq n$.

Usando que $\widehat{u}$ e $\widehat{v}$ pertencem a $\mathcal{E}_{M}\left[I \times \Omega^{\prime} ; \mathbb{R}^{n}\right]$ existem $\left.\bar{c}_{1}>0, \tau_{1} \in\right] 0, \tau\left[\right.$ e $N_{1} \in \mathbb{N}$ com $N_{1}>\max \left\{N, N n^{2}\left(b-t_{o}\right), N n^{2}\left(t_{o}-a\right)\right\}$ tais que

$$
\prod_{(\lambda, j) \in \Lambda \times A}\left|\partial^{\lambda} \widehat{u}_{j}(\varepsilon, t, y)\right| \leq \bar{c}_{1} \varepsilon^{-N_{1}} \quad \text { e } \prod_{(\lambda, j) \in \Lambda \times A}\left|\partial^{\lambda} \widehat{v}_{j}(\varepsilon, t, y)\right| \leq \bar{c}_{1} \varepsilon^{-N_{1}},
$$

para todo $\Lambda \times A \subset\left\{\theta \in \mathbb{N}^{m+1}:|\theta| \leq|\beta|\right\} \times\{1, \ldots, n\}$ e $\left.(\varepsilon, t, y) \in\right] 0, \tau_{1}\left[\times[a, b] \times K^{\prime}\right.$.

Como $\hat{h} \in \mathcal{N}\left[\stackrel{\circ}{K_{j}^{\prime}} ; \mathbb{R}^{n}\right]$ e $\hat{g} \in \mathcal{N}\left[\stackrel{\circ}{J_{j}} \times \stackrel{\circ}{K_{j}^{\prime}} ; \mathbb{R}^{n}\right]$, dados $q+2 N_{1}$ e $q+N_{1}$ existem $\bar{c}_{2}>0$ e $\left.\tau_{2} \in\right] 0, \tau_{1}[$ tais que

$$
\max \left\{\left|\widehat{h}_{i}(\varepsilon, y)\right|, \mid \partial^{\tilde{\mu}} \widehat{h}_{i}(\varepsilon, y) i\right\} \leq \bar{c}_{2} \varepsilon^{q+2 N_{1}} \quad \text { e } \quad\left|\partial^{\gamma} \widehat{g}_{i}(\varepsilon, t, y)\right| \leq \bar{c}_{2} \varepsilon^{q+N_{1}},
$$

para todo $\varepsilon \in] 0, \tau_{2}\left[, t \in[a, b], y \in K^{\prime}, \tilde{\mu} \in \mathbb{N}^{m}\right.$ com $|\tilde{\mu}| \leq|\beta|, \gamma \in \mathbb{N}^{m+1}$ com $|\gamma| \leq|\beta|$ e $1 \leq i \leq n$.

Seja $\left.\tau_{3} \in\right] 0, \tau_{2}\left[\right.$ tal que $0<\frac{1}{\ln \left(\bar{c} \varepsilon^{-N}\right)}<1$, para todo $\left.\varepsilon \in\right] 0, \tau_{3}[$.

Iremos agora começar a prova de (4).

Suponhamos $|\beta|=0$ e fixemos $\varepsilon \in] 0, \tau_{3}[$.

Usando (3), (6), e (10) concluímos que, se $(t, y) \in[a, b] \times K^{\prime}$, então

$$
\begin{aligned}
\|l(\varepsilon, t, y)\| & \leq\left|\int_{t_{o}}^{t}(\|\widehat{f}(\varepsilon, s, \widehat{v}(\varepsilon, s, y), y)-\widehat{f}(\varepsilon, s, \widehat{u}(\varepsilon, s, y), y)\|+|| \widehat{g}(\varepsilon, s, y)||) d s\right| \\
& \leq\left|\int_{t_{o}}^{t} \sum_{i=1}^{n}\left(\left|\widehat{f_{i}}(\varepsilon, s, \widehat{v}(\varepsilon, s, y), y)-\widehat{f}_{i}(\varepsilon, s, \widehat{u}(\varepsilon, s, y), y)\right|+\left|\widehat{g}_{i}(\varepsilon, s, y)\right|\right) d s\right| \\
& \leq\left|\int_{t_{o}}^{t}\left(n \ln \left(\bar{c} \varepsilon^{-N}\right)\|\widehat{v}(\varepsilon, s, y)-\widehat{u}(\varepsilon, s, y)\|+n \bar{c}_{2} \varepsilon^{q+N_{1}}\right) d s\right| \\
& \leq\left|\int_{t_{o}}^{t}\left(n \ln \left(\bar{c} \varepsilon^{-N}\right)|| l(\varepsilon, t, y)\left\|+n \ln \left(\bar{c} \varepsilon^{-N}\right)|| \widehat{h}(\varepsilon, y)\right\|+n \bar{c}_{2} \varepsilon^{q+N_{1}}\right) d s\right| \\
& \leq\left|\int_{t_{o}}^{t}\left(n \ln \left(\bar{c} \varepsilon^{-N}\right)|| l(\varepsilon, t, y) \|+n^{2} \bar{c}_{2} \bar{c} \varepsilon^{q+2 N_{1}-N}+n \bar{c}_{2} \varepsilon^{q+N_{1}}\right) d s\right|
\end{aligned}
$$




$$
\leq\left|\int_{t_{o}}^{t}\left(n \ln \left(\bar{c} \varepsilon^{-N}\right)\|l(\varepsilon, t, y)\|+n\left(\bar{c}_{2} \bar{c} n+\bar{c}_{2}\right) \varepsilon^{q+N_{1}}\right) d s\right|
$$

Portanto, pelo Lema de Gronwall (3.1.11), concluímos que, se $S$ e $\bar{c}_{2}$ são dados por

$$
\begin{aligned}
& S=\max \left\{\exp \left(n \ln \left(\bar{c} \varepsilon^{-N}\right)\left(b-t_{o}\right)\right)-1, \exp \left(n \ln \left(\bar{c} \varepsilon^{-N}\right)\left(t_{o}-a\right)\right)-1\right\} ; \\
& \bar{c}_{3}=\max \left\{\bar{c}^{n\left(b-t_{o}\right)}, \bar{c}^{n\left(t_{o}-a\right)}\right\}
\end{aligned}
$$

então

$$
\begin{aligned}
\|l(\varepsilon, t, y)\| & \leq \frac{(1+n \bar{c}) \bar{c}_{2} \varepsilon^{q+N_{1}}}{\ln \left(\bar{c} \varepsilon^{-N}\right)} S \\
& \leq \frac{(1+n \bar{c}) \bar{c}_{2} \varepsilon^{q+N_{1}}}{\ln \left(\bar{c} \varepsilon^{-N}\right)}\left(\bar{c}_{3} \varepsilon^{-N_{1}}-1\right) \\
& \leq \frac{(1+n \bar{c}) \bar{c}_{2} \varepsilon^{q+N_{1}}}{\ln \left(\bar{c} \varepsilon^{-N}\right)} \bar{c}_{3} \varepsilon^{-N_{1}} \leq(1+n \bar{c}) \bar{c}_{2} \bar{c}_{3} \varepsilon^{q}
\end{aligned}
$$

e assim, usando (10), para todo $(t, y) \in[a, b] \times K^{\prime}$ temos que

$$
\begin{aligned}
\|\widehat{v}(\varepsilon, t)-\widehat{u}(\varepsilon, t)\| & \leq \| \widehat{h}(\varepsilon, y\|+\| l(\varepsilon, t, y) \| \\
& \leq\|\widehat{h}(\varepsilon, y)\|+(1+n \bar{c}) \bar{c}_{2} \bar{c}_{3} \varepsilon^{q} \\
& \leq n \bar{c}_{2} \varepsilon^{q+2 N_{1}}+(1+n \bar{c}) \bar{c}_{2} \bar{c}_{3} \varepsilon^{q} \\
& \leq\left(n \bar{c}_{2}+(1+n \bar{c}) \bar{c}_{2} \bar{c}_{3}\right) \varepsilon^{q} .
\end{aligned}
$$

Como $\left|\widehat{v}_{i}(\varepsilon,)-.\widehat{u}_{i}(\varepsilon,).\right| \leq\|\widehat{v}(\varepsilon,)-.\widehat{u}(\varepsilon,)$.$\| temos que (4) é verdadeiro para |\beta|=0$.

Suponhamos $|\beta|>0$ e $\beta=\left(\beta_{0}, \beta_{1}, \ldots, \beta_{m}\right)$.

Denotaremos por $(t, y)=\left(t, y_{1}, \ldots, y_{m}\right)$ um ponto genérico de $\mathbb{R} \times \mathbb{R}^{m}$ e por $(t, x, y)=$ $\left(t, x_{1}, \ldots, x_{n}, y_{1}, \ldots, y_{m}\right)$ um ponto genérico de $\mathbb{R} \times \mathbb{R}^{n} \times \mathbb{R}^{m}$.

Usando a hipótese de indução (isto é, (4) $\operatorname{com} \beta$ substituído por $\gamma$, sendo $\gamma \in \mathbb{I}^{m+1}$ e $|\gamma|<|\beta|)$ e 3.1 .12 temos que, dado $3 N_{1}+q$ existem $\bar{c}_{4}>0$ e $\left.\tau_{4} \in\right] 0, \tau_{3}$ [ tais que

$$
\left|\prod_{(\lambda, j) \in \Lambda \times A} \partial^{\lambda} \widehat{u}_{j}(\varepsilon, t, y)-\prod_{(\lambda, j) \in \Lambda \times A} \partial^{\lambda} \widehat{v}_{j}(\varepsilon, t, y)\right| \leq \bar{c}_{4} \varepsilon^{3 N_{1}+q},
$$

para todo $\Lambda \times A \subset\left\{\theta \in \mathbb{N}^{m+1}:|\theta|<|\beta|\right\} \times\{1, \ldots, n\}$ e $\left.(\varepsilon, t, y) \in\right] 0, \tau_{4}\left[\times[a, b] \times K^{\prime}\right.$.

Fixemos $i \in\{1,2, \ldots, n\}$ e $\varepsilon \in] 0, \tau_{4}[$. Então usando (2) temos que $\widehat{v}_{i}(\varepsilon, t, y)-\widehat{u}_{i}(\varepsilon, t, y)=\widehat{h}_{i}(\varepsilon, y)+\int_{t_{0}}^{t}\left(\widehat{f}_{i}(\varepsilon, s, \widehat{v}(\varepsilon, s, y), y)-\widehat{f}_{i}(\varepsilon, s, \widehat{u}(\varepsilon, s, y), y)+\widehat{g}_{i}(\varepsilon, s, y)\right) d s$, 
e portanto, se

$$
w_{1}(\varepsilon, t, y)=\hat{f}_{i}(\varepsilon, t, \widehat{v}(\varepsilon, t, y), y) \quad \text { e } \quad w_{2}(\varepsilon, t, y)=\hat{f}_{i}(\varepsilon, t, \widehat{u}(\varepsilon, t, y), y)
$$

temos, por (3), para todo $(t, y) \in \stackrel{\circ}{J}_{j} \times \stackrel{\circ}{K}_{j}^{\prime}$, que

$$
\begin{aligned}
& \partial^{\beta} l_{i}(\varepsilon, t, y)=\int_{t_{0}}^{t} \partial^{\beta}\left(w_{1}-w_{2}+\widehat{g}_{i}\right)(\varepsilon, s, y) d s, \text { se } \beta_{0}=0 \\
& \partial^{\beta} l_{i}(\varepsilon, t, y)=\partial^{\bar{\beta}}\left(w_{1}-w_{2}+\widehat{g}_{i}\right)(\varepsilon, t, y), \text { se } \beta_{0}>0 \text { sendo } \bar{\beta}=\left(\beta_{0}-1, \beta_{1}, \ldots, \beta_{m}\right) .
\end{aligned}
$$

Portanto precisamos calcular $\partial^{\mu}\left(w_{1}-w_{2}\right)$, sendo $\mu \in \mathbb{N}^{m+1}$ com $|\mu| \leq|\beta|$.

Seja $\mu=\left(\mu_{0}, \mu_{1}, \ldots, \mu_{m}\right) \in \mathbb{N}^{m+1}$ com $|\mu| \leq|\beta|$. Então

$$
\begin{aligned}
& \partial^{\mu} w_{1}(\varepsilon, t, y)-\partial^{\mu} w_{2}(\varepsilon, t, y)=\bar{a}_{1}+\bar{a}_{2}+\bar{a}_{3}, \quad \text { sendo } \\
& \bar{a}_{1}=\partial^{\tilde{\gamma}} \widehat{f}_{i}(\varepsilon, t, \widehat{v}(\varepsilon, t, y), y)-\partial^{\tilde{\imath}} \widehat{f}_{i}(\varepsilon, t, \widehat{u}(\varepsilon, t, y), y)
\end{aligned}
$$

onde $\tilde{\gamma}=\left(\mu_{0}, \alpha_{1}, \ldots, \alpha_{n}, \mu_{1}, \ldots, \mu_{m}\right) \in \mathbb{N}^{n+m+1}$ com $\alpha_{j}=0$, para todo $1 \leq j \leq n$;

$$
\bar{a}_{2}=\sum_{k=1}^{n} \frac{\partial \widehat{f}_{i}}{\partial x_{k}}(\varepsilon, t, \widehat{v}(\varepsilon, t, y), y) \partial^{\mu} \widehat{v}_{k}(\varepsilon, t, y)-\sum_{k=1}^{n} \frac{\partial \widehat{f}_{i}}{\partial x_{k}}(\varepsilon, t, \widehat{u}(\varepsilon, t, y), y) \partial^{\mu} \widehat{u}_{k}(\varepsilon, t, y)
$$

e $\quad \bar{a}_{3}$ é soma de elementos do tipo

$$
\partial^{\gamma} \widehat{f}_{i}(\varepsilon, t, \widehat{v}(\varepsilon, t, y), y) \prod_{(\lambda, k) \in \Lambda \times A} \partial^{\lambda} \widehat{v}_{k}(\varepsilon, t, y)-\partial^{\gamma} \widehat{f}_{i}(\varepsilon, t, \widehat{u}(\varepsilon, t, y), y) \prod_{(\lambda, k) \in \Lambda \times A} \partial^{\lambda} \widehat{u}_{k}(\varepsilon, t, y),(16)
$$

$\operatorname{com} \gamma \in \mathbb{N}^{n+m+1},|\gamma| \leq|\mu|$ e $\Lambda \times A \subset\left\{\tilde{\alpha} \in \mathbb{N}^{m+1}:|\tilde{\alpha}|<|\mu|\right\} \times\{1, \ldots, n\}$

Usando que (15) pode ser escrita como $\bar{b}_{1}+\bar{b}_{2}$, sendo

$$
\begin{aligned}
& \bar{b}_{1}=\sum_{k=1}^{n}\left(\frac{\partial \widehat{f}_{i}}{\partial x_{k}}(\varepsilon, t, \widehat{v}(\varepsilon, t, y), y)-\frac{\partial \widehat{f}_{i}}{\partial x_{k}}(\varepsilon, t, \widehat{u}(\varepsilon, t, y), y)\right) \partial^{\mu} \widehat{v}_{k}(\varepsilon, t, y), \\
& \bar{b}_{2}=\sum_{k=1}^{n} \frac{\partial \widehat{f}_{i}}{\partial x_{k}}(\varepsilon, t, \widehat{u}(\varepsilon, t, y), y)\left(\partial^{\mu} \widehat{v}_{k}(\varepsilon, t, y)-\partial^{\mu} \widehat{u}_{k}(\varepsilon, t, y)\right)
\end{aligned}
$$

e que (16) pode ser escrita como $b_{\gamma \Lambda A}+c_{\gamma \Lambda A}$, sendo

$$
b_{\gamma \Lambda A}=\left[\partial^{\gamma} \widehat{f}_{i}(\varepsilon, t, \widehat{v}(\varepsilon, t, y), y)-\partial^{\gamma} \widehat{f}_{i}(\varepsilon, t, \widehat{u}(\varepsilon, t, y), y)\right] \prod_{(\lambda, k) \in \Lambda \times A} \partial^{\lambda} \widehat{v}_{k}(\varepsilon, t, y)
$$




$$
c_{\gamma \Lambda A}=\partial^{\gamma} \widehat{f}_{i}(\varepsilon, t, \widehat{u}(\varepsilon, t, y), y)\left[\prod_{(\lambda, k) \in \Lambda \times A} \partial^{\lambda} \widehat{v}_{k}(\varepsilon, t, y)-\prod_{(\lambda, k) \in \Lambda \times A} \partial^{\lambda} \widehat{u}_{k}(\varepsilon, t, y)\right]
$$

e que valem (5), (7), (8), (9) e (11), temos que

$$
\begin{aligned}
& \left|\bar{a}_{1}\right| \leq \bar{c} \varepsilon^{-N}\|\widehat{v}(\varepsilon, t, y)-\widehat{u}(\varepsilon, t, y)\| \leq n \bar{c}_{4} \bar{c} \varepsilon^{-N+3 N_{1}+q} \leq n \bar{c} \bar{c}_{4} \bar{c} \varepsilon^{2 N_{1}+q} \\
& \left|\bar{b}_{1}\right| \leq n \bar{c} \varepsilon^{-N}\|\widehat{v}(\varepsilon, t, y)-\widehat{u}(\varepsilon, t, y)\| \bar{c}_{1} \varepsilon^{-N_{1}} \leq n^{2} \bar{c}_{4} \bar{c}_{1} \bar{c} \varepsilon^{-N+3 N_{1}+q-N_{1}} \leq n^{2} \bar{c}_{4} \bar{c}_{1} \bar{c} \varepsilon^{N_{1}+q} \\
& \left|\bar{b}_{2}\right| \leq \sum_{k=1}^{n} \ln \left(\bar{c} \varepsilon^{-N}\right)\left|\partial^{\mu} \widehat{v}_{\dot{k}}(\varepsilon, t, y)-\partial^{\mu} \widehat{u}_{k}(\varepsilon, t, y)\right| ; \\
& \left|b_{\gamma \Lambda A}\right| \leq \bar{c} \varepsilon^{-N}\|\widehat{u}(\varepsilon, t, y)-\widehat{v}(\varepsilon, t, y)\| \bar{c}_{1} \varepsilon^{-N_{1}} \leq n \bar{c}_{4} \bar{c}_{1} \bar{c} \varepsilon^{-N+3 N_{1}+q-N_{1}} \\
& \left|c_{\gamma \Lambda A}\right| \leq \bar{c}_{4} \bar{c} \varepsilon^{-N+3 N_{1}+q} \leq \bar{c}_{4} \bar{c} \varepsilon^{N_{1}+q} .
\end{aligned}
$$

Como $\bar{a}_{3}$ é soma de elementos do tipo $b_{\gamma \Lambda A}+c_{\gamma \Lambda A}$ e valem (17) e (18) existe $\bar{c}_{5}>0$ tal que $\left|\bar{a}_{3}\right| \leq \bar{c}_{5} \varepsilon^{N_{1}+q}$, e assim

$$
\begin{aligned}
\left|\partial^{\mu} w_{1}(\varepsilon, t, y)-\partial^{\mu} w_{2}(\varepsilon, t, y)\right| \leq & \left|\bar{a}_{1}\right|+\left|\bar{a}_{2}\right|+\left|\bar{a}_{3}\right| \\
\leq & \left|\bar{a}_{1}\right|+\left|\bar{b}_{1}\right|+\left|\bar{a}_{3}\right|+\left|\bar{b}_{2}\right| \\
\leq & n \bar{c}_{4} \bar{c} \varepsilon^{q+N_{1}}+n^{2} \bar{c}_{4} \bar{c}_{1} \bar{c} \varepsilon^{q+N_{1}}+\bar{c}_{5} \varepsilon^{q+N_{1}}+ \\
& +\sum_{k=1}^{n} \ln \left(\bar{c} \varepsilon^{-N}\right)\left|\partial^{\mu} \widehat{v}_{k}(\varepsilon, t, y)-\partial^{\mu} \widehat{u}_{k}(\varepsilon, t, y)\right| .
\end{aligned}
$$

Se $\bar{c}_{6}=\max \left\{n^{2} \bar{c}_{4} \bar{c}_{1} \bar{c}, n \bar{c}_{4} \bar{c}, \bar{c}_{5}\right\}$ e $\tilde{\mu}=\left(\mu_{1}, \ldots, \mu_{m}\right)$ temos, denotando por $\partial^{\mu} l$ a n-upla $\left(\partial^{\mu} l_{1}, \ldots, \partial^{\mu} l_{n}\right)$, que

$$
\left|\partial^{\mu}\left(w_{1}-w_{2}\right)(\varepsilon, t, y)\right| \leq 3 \bar{c}_{6} \varepsilon^{q+N_{1}}+n \ln \left(\bar{c} \varepsilon^{-N}\right)|| \partial^{\mu} l\left(\varepsilon, t, y||+\sum_{j=1}^{n} \bar{c} \varepsilon^{-N}\left|\partial^{\tilde{\mu}} \widehat{h}_{i}(\varepsilon, y)\right|\right.
$$

e assim, por (10),

$$
\left|\partial^{\mu}\left(w_{1}-w_{2}\right)(\varepsilon, t, y)\right| \leq n \ln \left(\bar{c} \varepsilon^{-N}\right)\left\|\partial^{\mu} l(\varepsilon, t, y)\right\|+\left(3 \bar{c}_{6}+n \bar{c}_{2} \bar{c}\right) \varepsilon^{q+N_{1}}
$$

para $(\varepsilon, t, y) \in] 0, \tau_{4}\left[\times[a, b] \times K^{\prime}\right.$.

Suponhamos, em primeiro lugar, $\beta_{0}=0$. Então usando (10), (12) e (19) (fazendo $\mu=\beta)$ temos

$$
\left\|\partial^{\beta} l(\varepsilon, t, y)\right\| \leq \sum_{i=1}^{n}\left|\partial^{\beta} \widehat{l}_{i}(\varepsilon, t, y)\right|
$$




$$
\begin{aligned}
& \leq \sum_{i=1}^{n}\left|\int_{t_{o}}^{t}\left(n \ln \left(\bar{c} \varepsilon^{-N}\right)\left\|\partial^{\beta} l(\varepsilon, t, y)\right\|+\left(3 \bar{c}_{6}+n \bar{c}_{2} \bar{c}\right) \varepsilon^{q+N_{1}}+\partial^{\beta} \widehat{g}_{i}(\varepsilon, s, y)\right) d s\right| \\
& \leq\left|\int_{t_{o}}^{t}\left(n^{2} \ln \left(\bar{c} \varepsilon^{-N}\right)|| \partial^{\beta} l(\varepsilon, s, y) \|+n\left(\bar{c}_{2}+3 \bar{c}_{6}+n \bar{c}_{2} \bar{c}\right) \varepsilon^{q+N_{1}}\right) d s\right|
\end{aligned}
$$

e assim, pelo Lema de Gronwall (3.1.11), concluímos que, se $S$ e $\bar{c}_{7}$ são dados por

$$
\begin{aligned}
& S=\max \left\{\exp \left(n^{2} \ln \left(\bar{c} \varepsilon^{-N}\right)\left(b-t_{o}\right)\right)-1, \exp \left(n^{2} \ln \left(\bar{c} \varepsilon^{-N}\right)\left(t_{o}-a\right)\right)-1\right\} ; \\
& \bar{c}_{7}=\max \left\{\bar{c}^{n^{2}\left(b-t_{o}\right)}, \bar{c}^{n^{2}\left(t_{o}-a\right)}\right\},
\end{aligned}
$$

então

$$
\begin{aligned}
\left\|\partial^{\beta} l(\varepsilon, t, y)\right\| & \leq \frac{\left(\bar{c}_{2}+3 \bar{c}_{6}+n \bar{c}_{2} \bar{c}\right) \varepsilon^{q+N_{1}}}{n \ln \left(\bar{c} \varepsilon^{-N}\right)} S \\
& \leq\left(\bar{c}_{2}+3 \bar{c}_{6}+n \bar{c}_{2} \bar{c}\right) \bar{c}_{7} \varepsilon^{q+N_{1}-N_{1}} \\
& \leq\left(\bar{c}_{2}+3 \bar{c}_{6}+n \bar{c}_{2} \bar{c}\right) \bar{c}_{7} \varepsilon^{q} .
\end{aligned}
$$

Como, para $\tilde{\beta}=\left(\beta_{1}, \ldots, \beta_{m}\right)$, temos

$$
\begin{aligned}
\left|\partial^{\beta} \widehat{v}_{i}(\varepsilon, t, y)-\partial^{\beta} \widehat{u}_{i}(\varepsilon, t, y)\right| & \leq \mid ! \partial^{\beta} l(\varepsilon, t, y)\|+\| \partial^{\tilde{\beta}} \hat{h}(\varepsilon, y) \| \\
& \leq\left(\bar{c}_{2}+3 \bar{c}_{6}+n \bar{c}_{2} \bar{c}\right) \bar{c}_{7} \varepsilon^{q}+\sum_{k=1}^{n}\left|\partial^{\tilde{\beta}} \widehat{h}_{k}(\varepsilon, y)\right|
\end{aligned}
$$

e vale (10) temos que (4) é verdadeiro para $\beta \operatorname{com} \beta_{0}=0$.

Finalmente suponhamos $\beta_{0} \neq 0$ e seja $\bar{\beta}=\left(\beta_{0}-1, \beta_{1}, \ldots, \beta_{m}\right)$. Então usando (13), substituindo $\mu$ por $\bar{\beta}$ na desigualdade após a afirmação (18), e usando (10) e (11) temos que

$$
\begin{aligned}
\left|\partial^{\beta}\left(\widehat{v}_{i}-\widehat{u}_{i}\right)(\varepsilon, t, y)\right|= & \left|\partial^{\beta} l_{i}(\varepsilon, t, y)\right|=\left|\partial^{\bar{\beta}}\left(w_{1}-w_{2}+\widehat{g}_{i}\right)(\varepsilon, t, y)\right| \\
\leq & \left|\partial^{\bar{\beta}}\left(w_{1}-w_{2}\right)(\varepsilon, t, y)\right|+\left|\partial^{\bar{\beta}} \widehat{g}_{i}(\varepsilon, t, y)\right| \\
\leq & 3 \bar{c}_{6} \varepsilon^{q+N_{1}}+\sum_{k=1}^{n} \ln \left(\bar{c} \varepsilon^{-N}\right)\left|\partial^{\bar{\beta}}\left(\widehat{v}_{k}-\widehat{u}_{k}\right)(\varepsilon, t, y)\right|+ \\
& +\left|\partial^{\bar{\beta}} \widehat{g}_{i}(\varepsilon, t, y)\right| \\
\leq & 3 \bar{c}_{6} \varepsilon^{q+N_{1}}+\sum_{k=1}^{n} \bar{c} \varepsilon^{-N} \bar{c}_{4} \varepsilon^{3 N_{1}+q}+\bar{c}_{2} \varepsilon^{q+N_{1}} \\
\leq & \left(3 \bar{c}_{6}+n \bar{c}_{4} \bar{c}+\bar{c}_{2}\right) \varepsilon^{q}
\end{aligned}
$$


para todo $(\varepsilon, t, y) \in] 0, \tau_{4}\left[\times[a, b] \times K^{\prime}\right.$, e assim concluímos que (2) é verdadeiro nesse caso.

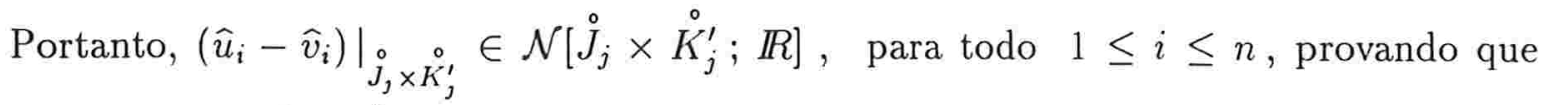
$\left.(\widehat{u}-\widehat{v})\right|_{J_{j} \times K_{j}^{\prime}} \in \mathcal{N}\left[\stackrel{\circ}{J}_{j} \times{\stackrel{\circ}{K_{j}^{\prime}}}_{j} ; \mathbb{R}^{n}\right] . / /$

Finalizamos esta seção com os seguintes teoremas:

3.2.18 Teorema(Existência $\varepsilon$ Unicidade). Sejam $\Omega$ um aberto de $\mathbb{R}^{n}$, I um intervalo aberto de $\mathbb{R}, t_{o} \in I, \Omega^{\prime}$ um aberto de $\mathbb{R}^{m}, W$ um aberto de $\Omega^{\prime}, g \in \mathcal{G}\left(W ; \mathbb{R}^{n}\right)$, $\hat{g}$ um representante de $g, f \in \mathcal{G}\left(I \times \Omega \times \Omega^{\prime} ; \mathbb{R}^{n}\right)$ e $\hat{f}=\left(\hat{f}_{1}, \ldots, \hat{f}_{n}\right)$ um representante de $f$. Denotando por $(t, x, y)=\left(t, x_{1}, \ldots, x_{n}, y_{1}, \ldots, y_{m}\right)$ um ponto genérico de $I \times \Omega \times \Omega^{\prime}$ tem-se que, se

(i) $\bar{W} \subset \subset \Omega^{\prime}$;

(ii) existem $U$ aberto de $\Omega$ e $\left.\left.\tau_{1} \in\right] 0,1\right]$ tais que $\bar{U} \subset \subset \Omega$ e $\hat{g}(] 0, \tau_{1}[\times W) \subset U$;

(iii) $g \in \mathcal{G}_{*}(W ; U)$;

(iv) $f \in \mathcal{G}_{*}\left(I \times \Omega \times \Omega^{\prime} ; \mathbb{R}^{n}\right)$;

(v) $\frac{\partial \hat{f}_{i}}{\partial x_{j}}$ tem a propriedade (CLL) em $I \times \Omega \times \Omega^{\prime}$, para todo $1 \leq i, j \leq n$;

(vi) $\widehat{f}$ tem a propriedade (LLL) em $\left(I, \Omega, \Omega^{\prime}\right)$,

então existe $a>0$ com $\overline{I_{a}\left(t_{o}\right)} \subset I$ e existe uma única função $u \in \mathcal{G}_{*}\left(I_{a}\left(t_{o}\right) \times W ; \Omega\right)$ satisfazendo

$$
\frac{\partial u}{\partial t}=f \circ\left(\pi, u, \pi_{1}, \ldots, \pi_{m}\right) \quad \text { e }\left.\quad u\right|_{\left\{t_{o}\right\} \times W}=g .
$$

Demonstração. É uma conseqüência de 3.2.12 juntamente com 3.2.17. //

3.2.19 Teorema. Se $\Omega, I, t_{o}, \Omega^{\prime}, g, f, \hat{g}$ e $\hat{f}$ são como em 3.2.13(ou 3.2.14 ou 3.2.15) e se $\hat{f}$ tem a propriedade (LLL) em $\left(I, \Omega, \Omega^{\prime}\right)$, entâo existe $a>0$ com $\overline{I_{a}\left(t_{o}\right)} \subset I$ e existe uma única função $u \in \mathcal{G}_{*}\left(I_{a}\left(t_{0}\right) \times \Omega^{\prime} ; \Omega\right)$ satisfazendo

$$
\frac{\partial u}{\partial t}=f \circ\left(\pi, u, \pi_{1}, \ldots, \pi_{m}\right) \quad \text { e }\left.\quad u\right|_{\left\{t_{o}\right\} \times \Omega^{\prime}}=g .
$$


Demonstração. Basta usar 3.2.17 e 3.2.13(ou 3.2.14 ou 3.2.15). //

Completamos o resultado acima com o seguinte exemplo:

3.2.20 Exemplo. Sejam I, $\Omega^{\prime},\left(\psi_{i}\right)_{1 \leq i \leq n},\left(\Psi_{i}\right)_{1 \leq i \leq n},\left(\varphi_{i}\right)_{1 \leq i \leq n},\left(\mu_{i}\right)_{1 \leq i \leq n},\left(\nu_{i}\right)_{1 \leq i \leq n}$ e $\hat{f}=\left(\hat{f}_{1}, \ldots, \hat{f}_{n}\right)$ como em 3.2.16. Então $\hat{f}$ satisfaz de 3.2.18.iv até 3.2.18.vi(substituindo $\Omega$ por $\left.\mathbb{R}^{n}\right)$.

(Basta usar 3.2.16 e observar que a prova de que $\widehat{f}$ satisfaz 3.2.18.vi é análoga à de 3.1.17.)

\subsection{Equação de Hamilton-Jacobi : unicidade de so- luções}

Aqui usaremos as notações e alguns fatos do capítulo 2, principalmente 2.1.3 que recapitularemos a seguir.

Sejam $\Omega$ e $\Omega^{\prime}$ abertos de $\mathbb{R}^{n}, I$ e $J$ intervalos abertos de $\mathbb{R}$ com $0 \in J \subset I, W$ um aberto de $\mathbb{R}^{n+1}$ com $V=\left\{z \in \mathbb{R}^{n}:(0, z) \in W\right\} \neq \emptyset$ e $W \subset I \times \Omega, H \in \mathcal{G}\left(I \times \Omega \times \Omega^{\prime} ; \mathbb{R}\right)$ e $f \in \mathcal{G}(\Omega ; \mathbb{R})$ tais que $\mathcal{S}\left(I, \Omega, \Omega^{\prime}, H, f, J, W\right) \neq \emptyset$. Se $(t, x, p)=\left(t, x_{1}, \ldots, x_{n}, p_{1}, \ldots, p_{n}\right)$ denota um ponto genérico de $I \times \Omega \times \Omega^{\prime},(t, x)=\left(t, x_{1}, \ldots, x_{n}\right)$ denota um ponto genérico de $W,(X, P) \in \mathcal{S}\left(I, \Omega, \Omega^{\prime}, H, f, J, W\right), Y=(\pi, X), P=\left(P_{1}, \ldots, P_{n}\right)$ e $U \in \mathcal{G}(J \times V ; \mathbb{R})$ é tal que

$$
\frac{\partial U}{\partial s}=-H \circ(\pi, X, P)+\sum_{j=1}^{n}\left(\frac{\partial H}{\partial p_{j}} \circ(\pi, X, P)\right) P_{j} \quad \text { e }\left.\quad U\right|_{\{0\} \times V}=\left.f\right|_{V}
$$

então

$$
\begin{aligned}
& u=U \circ Y^{-1} \text { é uma solução para o problema HJ em } \mathcal{G}(W ; \mathbb{R}) ; \\
& \left(\frac{\partial u}{\partial x_{1}}, \ldots, \frac{\partial u}{\partial x_{n}}\right) \circ Y=P \quad \text { em } \mathcal{G}\left(J \times V ; \mathbb{R}^{n}\right) .
\end{aligned}
$$

Uma questão que surge naturalmente é: 
Se $\mathcal{S}\left(I, \Omega, \Omega^{\prime}, H, f, J, W\right) \neq \emptyset$, então existe uma única solução para o problema $\mathbf{H J}$ ?

Procurando obter alguma resposta para a questão acima chegamos aos resultados que estão nesta seção a partir de 3.3.3. Em 3.3.1 apresentamos, com o auxílio 3.2.14, mais exemplos de funções $H$ e $f$ para as quais o problema HJ correspondente admite solução.

3.3.1 Teorema. Sejam $I$ uin intervalo aberto de $\mathbb{R}$ com $0 \in I, f \in \mathcal{G}\left(\mathbb{R}^{n} ; \mathbb{R}\right), H \in$ $\mathcal{G}\left(I \times \mathbb{R}^{n} \times \mathbb{R}^{n} ; \mathbb{R}\right), \hat{f}$ um representante de $f$ e $\widehat{H}$ um representante de $H$. Se

(i) $\nabla f \in \mathcal{G}_{*}\left(\mathbb{R}^{n} ; \mathbb{R}^{n}\right)$;

(ii) $\partial^{\alpha} \widehat{f}$ é uma função limitada em $\mathbb{R}^{n}$, para todo $\alpha \in \mathbb{N}^{n}$ com $|\alpha|=2$;

(iii) $\partial^{\gamma} \widehat{H} \quad$ é uma função limitada em $I \times \mathbb{R}^{n} \times \mathbb{R}^{n}, \quad$ para todo $\gamma=\left(\gamma_{0}, \gamma_{1}, \ldots, \gamma_{2 n}\right) \in$ $\mathbb{N}^{2 n+1}$ com $\gamma_{0}=0<|\gamma| \leq 2$ ou $\gamma_{j}=0<\gamma_{0}=1<|\gamma|=2$ para todo $1 \leq j \leq n$,

então existe $a>0$ com $\overline{I_{a}} \subset I$ e $\mathcal{S}\left(I, \mathbb{R}^{n}, \mathbb{R}^{n}, H, f, I_{a}, I_{a} \times \mathbb{R}^{n}\right) \neq \emptyset$, e em conseqüência existe $u \in \mathcal{G}\left(I_{a} \times \mathbb{R}^{n} ; \mathbb{R}\right)$ tal que

(I) u é uma solução para o problema $\mathbf{H J}$ em $\mathcal{G}\left(I_{a} \times \mathbb{R}^{n} ; \mathbb{R}\right)$.

Se $(t, x)=\left(t, x_{1}, \ldots, x_{n}\right)$ denota um ponto genérico de $I_{a} \times \mathbb{R}^{n}$, então u também satisfaz

(II) existe um representante $\widehat{v}=\left(\widehat{v}_{1}, \ldots, \widehat{v}_{n}\right)$ de $\left(\frac{\partial u}{\partial x_{1}}, \ldots, \frac{\partial u}{\partial x_{n}}\right)$ tal que $\frac{\partial \widehat{v}_{i}}{\partial x_{j}}$ é uma função limitada em $I_{a} \times \mathbb{R}^{n}$, para todo $1 \leq i, j \leq n$;

(III) $\frac{\partial^{2} u}{\partial x_{i} \partial x_{j}} \in \mathcal{G}_{*}\left(I_{a} \times \mathbb{R}^{n} ; \mathbb{R}\right), \quad$ para todo $1 \leq i, j \leq n$.

Demonstração. Provaremos, em primeiro lugar, que existe $a>0$ satisfazendo (ver 2.1.1)

$$
\mathcal{S}\left(I, \mathbb{R}^{n}, \mathbb{R}^{n}, H, f, I_{a}, I_{a} \times \mathbb{R}^{n}\right) \neq \emptyset
$$

e assim com o auxílio de 2.1.3, como vimos no início desta seção, obteremos (I). 
Denotaremos por $(s, x, p, r)=\left(s, x_{1}, \ldots, x_{n}, p_{1}, \ldots, p_{n}, r_{1}, \ldots, r_{n}\right)$ um ponto genérico de $I \times \mathbb{R}^{3 n}$, por $(s, r)=\left(s, r_{1}, \ldots, r_{n}\right)$ um ponto genérico de $\mathbb{R}^{n+1}$ e por $r=\left(r_{1}, \ldots, r_{n}\right)$ um ponto genérico de $\mathbb{R}^{n}$.

Sejam $\hat{\varphi}$ a aplicação moderada definida em $] 0,1] \times I \times \mathbb{R}^{n} \times \mathbb{R}^{n} \times \mathbb{R}^{n}$ por

$$
\hat{\varphi}(\varepsilon, s, x, p, r)=\left(\frac{\partial \widehat{H}}{\partial p}(\varepsilon, s, x, p),-\frac{\partial \widehat{H}}{\partial x}(\varepsilon, s, x, p)\right),
$$

$\varphi$ a classe de $\hat{\varphi}=\left(\hat{\varphi}_{1}, \ldots, \hat{\varphi}_{2 n}\right)$ em $\mathcal{G}\left(I \times \mathbb{R}^{3 n} ; \mathbb{R}^{2 n}\right), \hat{g}$ a aplicação moderada definida em ] $0,1] \times \mathbb{R}^{n}$ por

$$
\widehat{g}(\varepsilon, r)=\left(r, \frac{\partial \hat{f}}{\partial r_{1}}(\varepsilon, r), \ldots, \frac{\partial \hat{f}}{\partial r_{n}}(\varepsilon, r)\right)
$$

e $g$ a classe de $\hat{g}$ em $\mathcal{G}\left(\mathbb{R}^{n} ; \mathbb{R}^{2 n}\right)$.

Sejam $\pi, \pi_{1}, \ldots, \pi_{n}$ como no início do capítulo 2 e consideremos, em $\mathcal{G}\left(I \times \mathbb{R}^{n}\right)$, o sistema de incógnitas $X$ e $P$

$$
\left(\frac{\partial X}{\partial s}, \frac{\partial P}{\partial s}\right)=\varphi \circ\left(\pi, X, P, \pi_{1}, \ldots, \pi_{n}\right)
$$

com a condição

$$
\left.(X, P)\right|_{\{0\} \times R^{n}}=g .
$$

Usando (ii) e (iii) existem $M>0$ e $\eta \in] 0,1]$ tais que

$$
\left.\left|\partial^{\alpha} \widehat{f}(\varepsilon, r)\right| \leq M, \text { para todo }(\varepsilon, r) \in\right] 0, \eta\left[\times \mathbb{R}^{n} \quad \text { e }|\alpha|=2\right.
$$

$\left|\partial^{\gamma} \widehat{H}(\varepsilon, t, x, p)\right| \leq M$, para todo $\left.(\varepsilon, t, x, p) \in\right] 0, \eta\left[\times I \times \mathbb{R}^{n} \times \mathbb{R}^{n}\right.$ e $\gamma$ como em (iii).

De (i) temos que $g \in \mathcal{G}_{*}\left(\mathbb{R}^{n} ; \mathbb{R}^{2 n}\right)$ e de (2) concluímos que $\hat{\varphi}, \frac{\partial \widehat{\varphi}_{i}}{\partial x_{j}}, \frac{\partial \hat{\varphi}_{i}}{\partial p_{j}}$ são limitadas em $I \times\left(\mathbb{R}^{n} \times \mathbb{R}^{n}\right) \times \mathbb{R}^{n}$, para todo $1 \leq i \leq 2 n$ e $1 \leq j \leq n$.

Portanto, por 3.1.2.II e 3.2.14, existem $a^{*}>0$ com $\overline{I_{a^{*}}} \subset I$ e uma aplicação $(X, P) \in$ $\mathcal{G}\left(I_{a} \cdot \times \mathbb{R}^{n} ; \mathbb{R}^{2 n}\right)$ tais que

$$
(X, P) \in \mathcal{G}_{*}\left(I_{a^{*}} \times \mathbb{R}^{n} ; \mathbb{R}^{2 n}\right) ;
$$

existem $\widehat{X}=\left(\widehat{X}_{1}, \ldots, \widehat{X}_{n}\right)$ representante de $X, \widehat{P}=\left(\widehat{P}_{1}, \ldots, \widehat{P}_{n}\right)$ representante de $P$ e $\tau \in] 0, \eta[$ tais que 


$$
\begin{aligned}
& \frac{\partial \widehat{X}}{\partial s}(\varepsilon, s, r)=\frac{\partial \widehat{H}}{\partial p}(\varepsilon, s, \widehat{X}(\varepsilon, s, r), \widehat{P}(\varepsilon, s, r)) ; \\
& \frac{\partial \widehat{P}}{\partial s}(\varepsilon, s, r)=-\frac{\partial \widehat{H}}{\partial x}(\varepsilon, s, \widehat{X}(\varepsilon, s, r), \widehat{P}(\varepsilon, s, r)) ; \\
& \widehat{X}(\varepsilon, 0, r)=r \quad \text { e } \quad \hat{P}(\varepsilon, 0, r)=\left(\frac{\partial \widehat{f}}{\partial r_{1}}(\varepsilon, r), \ldots, \frac{\partial \widehat{f}}{\partial r_{n}}(\varepsilon, r)\right),
\end{aligned}
$$

para todo $(\varepsilon, s, r) \in] 0, \tau\left[\times I_{a^{*}} \times \mathbb{R}^{n}\right.$ e ainda, por (1) e 3.2.14, para todo $\left.b \in\right] 0, a^{*}[$ temos que

$$
\partial^{\alpha} \widehat{X}_{i} \quad \text { e } \quad \partial^{\alpha} \widehat{P}_{i} \text { são limitadas em } \overline{I_{b}} \times \mathbb{R}^{n},
$$

para todo $\alpha=\left(\alpha_{0}, \alpha_{1}, \ldots, \alpha_{n}\right) \in \mathbb{N}^{n+1}$ com $\alpha_{0}=0<|\alpha|=1$ e $1 \leq i \leq n$.

Usando (4) e (6) temos que

$$
\widehat{X}(\varepsilon, s, r)=r+\int_{0}^{s} \frac{\partial \widehat{H}}{\partial p}(\varepsilon, t, \widehat{X}(\varepsilon, t, r), \widehat{P}(\varepsilon, t, r)) d t,
$$

para todo $(\varepsilon, s, r) \in] 0, \tau\left[\times I_{a^{\bullet}} \times \mathbb{R}^{n}\right.$.

Sejam $b>0$ com $\overline{I_{b}} \subset I_{a^{*}}, \widehat{Y}=(\pi, \widehat{X}), Y$ a classe de $\hat{Y}$ em $\mathcal{G}\left(I_{b} \times \mathbb{R}^{n} ; \mathbb{R}^{n+1}\right)$ e $\hat{l}$ a aplicação moderada definida em $] 0,1] \times I_{b} \times \mathbb{R}^{n}$ por

$$
\widehat{l}(\varepsilon, s, r)=\int_{0}^{s} \frac{\partial \widehat{H}}{\partial p}(\varepsilon, t, \widehat{X}(\varepsilon, t, r), \widehat{P}(\varepsilon, t, r)) d t .
$$

Provaremos que $\hat{l}=\left(\hat{l}_{1}, \ldots, \widehat{l}_{n}\right)$ satisfaz as hipóteses de 1.2.22(substituindo $\widehat{g}_{i}$ por $\left.\hat{l}_{i}\right)$.

Notemos que

$$
\begin{aligned}
& \frac{\partial \widehat{l}_{i}}{\partial s}(\varepsilon, s, r)=\frac{\partial \widehat{H}}{\partial p_{i}}(\varepsilon, s, \widehat{X}(\varepsilon, s, r), \widehat{P}(\varepsilon, s, r)) ; \\
& \frac{\partial^{2} \widehat{l}_{i}}{\partial s^{2}}(\varepsilon, s, r)= \frac{\partial^{2} \widehat{H}}{\partial t \partial p_{i}}(\varepsilon, s, \widehat{X}(\varepsilon, s, r), \widehat{P}(\varepsilon, s, r))+ \\
&+\sum_{j=1}^{n} \frac{\partial^{2} \widehat{H}}{\partial x_{j} \partial p_{i}}(\varepsilon, s, \widehat{X}(\varepsilon, s, r), \widehat{P}(\varepsilon, s, r)) \frac{\partial \widehat{X}_{j}}{\partial s}(\varepsilon, s, r)+ \\
&+\sum_{j=1}^{n} \frac{\partial^{2} \widehat{H}}{\partial p_{j} \partial p_{i}}(\varepsilon, s, \widehat{X}(\varepsilon, s, r), \widehat{P}(\varepsilon, s, r)) \frac{\partial \widehat{P}_{j}}{\partial s}(\varepsilon, s, r) ; \\
& \frac{\partial^{2} \widehat{l}_{i}}{\partial r_{k} \partial s}(\varepsilon, s, r)= \sum_{j=1}^{n} \frac{\partial^{2} \widehat{H}}{\partial x_{j} \partial p_{i}}(\varepsilon, s, \widehat{X}(\varepsilon, s, r), \widehat{P}(\varepsilon, s, r)) \frac{\partial \widehat{X}_{j}}{\partial r_{k}}(\varepsilon, s, r)+
\end{aligned}
$$




$$
+\sum_{j=1}^{n} \frac{\partial^{2} \widehat{H}}{\partial p_{j} \partial p_{i}}(\varepsilon, s, \widehat{X}(\varepsilon, s, r), \widehat{P}(\varepsilon, s, r)) \frac{\partial \widehat{P}_{j}}{\partial r_{k}}(\varepsilon, s, r),
$$

e assim usando (2), (4), (5) e (7) concluímos que as funções $\frac{\partial \widehat{l}_{i}}{\partial s}, \frac{\partial^{2} \widehat{l}_{i}}{\partial s^{2}}, \frac{\partial \widehat{l}_{i}}{\partial r_{k} \partial s}$ são limitadas em $I_{b} \times \mathbb{R}^{n}$, para todo $1 \leq i, k \leq n$, que é a afirmação 1.2.22.ii.

Como $\hat{l}_{i}(\varepsilon, 0,)=$.0 para todo $\left.\left.\varepsilon \in\right] 0,1\right]$ e $1 \leq i \leq n$, concluímos, por 1.2 .22 , que existe $a>0$ com $\overline{I_{a}} \subset I_{b}$ e tal que se verificam as afirmações abaixo:

$\left.Y\right|_{I_{a} \times R^{n}} \in \mathcal{G}_{*}\left(I_{a} \times \mathbb{R}^{n} ; I_{a} \times \mathbb{R}^{n}\right)$

$\left.Y\right|_{I_{a} \times R^{n}}$ é uma aplicação inversível;

$$
\left.\inf \{|J \hat{Y}(\varepsilon, s, r)|:(\varepsilon, s, r) \in] 0, \bar{\eta}\left[\times I_{a} \times \mathbb{R}^{n}\right\}>0, \text { para algum } \bar{\eta} \in\right] 0, \tau[
$$

existe um representante $\widehat{\Gamma}=\left(\widehat{\Gamma}_{0}, \widehat{\Gamma}_{1}, \ldots, \widehat{\Gamma}_{n}\right)$ de $\left(\left.Y\right|_{I_{a} \times R^{n}}\right)^{-1}$ tal que

$$
\left.\widehat{\Gamma}(\varepsilon, .)=\left(\left.\widehat{Y}(\varepsilon, .)\right|_{I_{a} \times R^{n}}\right)^{-1}, \text { para todo } \varepsilon \in\right] 0, \bar{\eta}[
$$

e para todo $\alpha \in \mathbb{N}^{n+1}$ com $|\alpha|=1$ e $1 \leq i \leq n$ tem-se que

$$
\partial^{\alpha} \widehat{\Gamma}_{i} \text { é uma função limitada em } I_{a} \times \mathbb{R}^{n} \text {. }
$$

Usando (3), (4), (5), (6), (8) e (9) concluímos que

$$
(X, P) \in \mathcal{S}\left(I, \mathbb{R}^{n}, \mathbb{R}^{n}, H, f, I_{a}, I_{a} \times \mathbb{R}^{n}\right),
$$

e assim, como vimos no início desta seção (afirmações $(\mathbf{A})$ e $(\mathbf{B})$ ), temos que

$$
\begin{gathered}
u=U \circ\left(\left.Y\right|_{I_{a} \times R^{n}}\right)^{-1} \text { é uma solução para o problema } \mathbf{H J} \text { em } \mathcal{G}\left(I_{a} \times \mathbb{R}^{n} ; \mathbb{R}\right) ; \\
\left(\frac{\partial u}{\partial x_{1}}, \ldots, \frac{\partial u}{\partial x_{n}}\right)=P \circ\left(\left.Y\right|_{I_{a} \times R^{n}}\right)^{-1},
\end{gathered}
$$

e $\operatorname{assim}(\mathrm{I})$ é verdadeira.

Seja $\widehat{v}=\widehat{P} \circ \widehat{\Gamma}$, sendo $\widehat{\Gamma}$ como em (11). Então $\widehat{v}=\left(\widehat{v}_{1}, \ldots, \widehat{v}_{n}\right)$ é um representante de $\left(\frac{\partial u}{\partial x_{1}}, \ldots, \frac{\partial u}{\partial x_{n}}\right)$. Provaremos que $\hat{v}$ satisfaz (II).

Notemos que, se $1 \leq i, k \leq n$ e $(\varepsilon, t, x) \in] 0,1] \times I_{a} \times \mathbb{R}^{n}$, então

$$
\frac{\partial \widehat{v}_{i}}{\partial x_{k}}(\varepsilon, t, x)=\frac{\partial}{\partial x_{k}}\left(\widehat{P}_{i} \circ \hat{\Gamma}\right)(\varepsilon, t, x)
$$




$$
=\frac{\partial \widehat{P}_{i}}{\partial s}(\varepsilon, \widehat{\Gamma}(\varepsilon, t, x)) \frac{\partial \widehat{\Gamma}_{0}}{\partial x_{k}}(\varepsilon, t, x)+\sum_{j=1}^{n} \frac{\partial \hat{P}_{i}}{\partial r_{j}}(\varepsilon, \widehat{\Gamma}(\varepsilon, t, x)) \frac{\partial \widehat{\Gamma}_{j}}{\partial x_{k}}(\varepsilon, t, x),
$$

e assim por (2), (5), (7) e (12) temos (II).

A asserção (III) é uma conseqüência imediata de (II). //

Apresentamos, a seguir, algumas funções que verificam as hipóteses de 3.3.1.

3.3.2 Exemplo. Sejam $I$ um intervalo aberto de $\mathbb{R}$ com $0 \in I, 1 \leq j \leq n, \mu_{j}, \nu_{j} e$ $\tilde{\nu}_{j}$ funções definidas em $\left.] 0,1\right]$ e com valores em $\mathbb{R}$ e $\varphi_{j}, \psi_{j}$ e $\Phi_{j}$ funções pertencentes a $\mathcal{C}^{\infty}(\mathbb{R} ; \mathbb{R})$ tais que $\mu_{j}, \nu_{j}, \tilde{\nu}, \varphi_{j}: \psi_{j}^{\prime}, \psi_{j}^{\prime \prime}$ e $\Phi_{j}$ são funções limitadas. Se $\hat{f}$ é a aplicação moderada definida em $] 0,1] \times \mathbb{R}^{n}$ por

$$
\widehat{f}\left(\varepsilon, r_{1}, \ldots, r_{n}\right)=\sum_{j=1}^{n} \int_{0}^{r_{j}}\left(\int_{0}^{s} \varphi_{j}\left(\mu_{j}(\varepsilon) y\right) d y\right) d s
$$

e $\widehat{H}$ é a aplicação moderada definida $\mathrm{em}] \mathrm{G}, 1] \times I \times \mathbb{R}^{n} \times \mathbb{R}^{n}$ por

$$
\widehat{H}\left(\varepsilon, t, x_{1}, \ldots, x_{n}, y_{1}, \ldots, y_{n}\right)=\sum_{j=1}^{n} \psi_{j}\left(\nu_{j}(\varepsilon)\left(t+x_{j}+p_{j}\right)\right)
$$

ou por

$$
\widehat{H}\left(\varepsilon, t, x_{1}, \ldots, x_{n}, y_{1}, \ldots, y_{n}\right)=\sum_{j=1}^{n} \nu_{j}(\varepsilon) \psi_{j}\left(\left(t+x_{j}+p_{j}\right)\right),
$$

ou por, se $\bar{I} \subset \subset \mathbb{R}, \inf \{|\tilde{\nu}(\varepsilon)|: \varepsilon \in] 0,1]\}>0$ e $\Phi_{j}$ e $\Phi_{j}^{\prime}$ pertencem a $L_{1}(\mathbb{R})$, para todo $1 \leq j \leq n$,

$$
\widehat{H}\left(\varepsilon, t, x_{1}, \ldots, x_{n}, p_{1}, \ldots, p_{n}\right)=\sum_{j=1}^{n} t \int_{0}^{p_{j}}\left(\int_{0}^{x_{j}} \Phi_{j}\left(\tilde{\nu}_{j}(\varepsilon)(s+y)\right) d s\right) d y,
$$

então $\hat{f}, \widehat{H}$, a classe de $\widehat{f}$ em $\mathcal{G}\left(\mathbb{R}^{n} ; \mathbb{R}\right)$ e a classe de $\widehat{H}$ em $\mathcal{G}\left(I \times \mathbb{R}^{n} \times \mathbb{R}^{n} ; \mathbb{R}\right)$ satisfazem as hipóteses de 3.3.1.

(Podemos, por exemplo, escolher para $\varphi_{j}$ a função $l_{1}(x)=\operatorname{sen}\left(x^{2}\right)$, para $\psi_{j}$ a função $l_{2}(x)=\operatorname{sen} x$ e para $\Phi_{j}$ a função $l_{3}(x)=\exp \left(-x^{2}\right)$.)

A seguir apresentaremos as respostas que obtivemos para a questão feita no início desta seção e com as quais completamos este trabalho. 
3.3.3 Teorema. Sejam $\Omega$ e $\Omega^{\prime}$ abertos de $\mathbb{R}^{n}, I$ e $J$ intervalos abertos de $\mathbb{R}$ com $0 \in J \subset I, W$ um aberto de $\mathbb{R}^{n+1} \operatorname{com} V=\left\{z \in \mathbb{R}^{n}:(0, z) \in W\right\} \neq \emptyset$ e $W \subset I \times \Omega$, $H \in \mathcal{G}\left(I \times \Omega \times \Omega^{\prime} ; \mathbb{R}\right)$ e $f \in \mathcal{G}(\Omega ; \mathbb{R})$. Se existe um representante $\widehat{H}$ de $H$ tal que

(i) $\frac{\partial^{2} \widehat{H}}{\partial x_{j} \partial p_{i}}, \frac{\partial^{2} \widehat{H}}{\partial x_{j} \partial x_{i}}$ e $\frac{\partial^{2} \widehat{H}}{\partial p_{j} \partial p_{i}}$ têm a propriedade (CLL) em $I \times \Omega \times \Omega^{\prime}$, para todo $1 \leq i, j \leq n$

(ii) $\frac{\partial \widehat{H}}{\partial x} \quad e-\frac{\partial \widehat{H}}{\partial p}$ têm a propriedade (LLL) em $\left(I, \Omega \times \Omega^{\prime}\right)$,

então

(I) $\operatorname{card} \mathcal{S}\left(I, \Omega, \Omega^{\prime}, H, f, J, W\right) \leq 1$;

(II) existe no máximo uma solução, construída como em (A) (ver início desta seção), para o problema $\mathbf{H J}$ em $\mathcal{G}(W ; \mathbb{R})$.

Demonstração. De (I) e 2.1.3 obtemos (II). Provaremos (I).

Sejam $\hat{\varphi}$ a aplicação moderada definida em $] 0,1] \times J \times\left(\Omega \times \Omega^{\prime}\right) \times V$ por

$$
\hat{\varphi}(\varepsilon, t, x, p, r)=\left(\frac{\partial \widehat{H}}{\partial p}(\varepsilon, t, x, p),-\frac{\partial \widehat{H}}{\partial x}(\varepsilon, t, x, p)\right)
$$

e $\varphi$ a classe de $\hat{\varphi}$ em $\mathcal{G}\left(J \times \Omega \times \Omega^{\prime} \times V ; \mathbb{R}^{2 n}\right)$.

Suponhamos $\operatorname{card} \mathcal{S}\left(I, \Omega, \Omega^{\prime}, H, f, J, W\right)>0$ e sejam $(X, P)$ e $(\tilde{X}, \tilde{P})$ pertencentes a $\mathcal{S}\left(I, \Omega, \Omega^{\prime}, H, f, J, W\right)$. Então, por 2.1.1, temos que $(X, P)$ e $(\tilde{X}, \tilde{P})$ pertencem a $\mathcal{G}_{*}(J \times$ $\left.V ; \Omega \times \Omega^{\prime}\right)$ e são soluções da equação nas incógnitas $(\bar{X}, \bar{P})$

$$
\left(\frac{\partial \bar{X}}{\partial s}, \frac{\partial \bar{P}}{\partial s}\right)=\varphi \circ\left(\pi, \bar{X}, \bar{P}, \pi_{1}, \ldots, \pi_{n}\right)
$$

com a condição

$$
\left.(\bar{X}, \bar{P})\right|_{\{0\} \times V}=\left(1_{V},\left.\nabla f\right|_{V}\right) .
$$

Usando (i) e (ii) temos que $\hat{\varphi}$ tem a propriedade (LLL) em $\left(J, \Omega \times \Omega^{\prime}, V\right)$ e $\partial^{\gamma} \hat{\varphi}_{i}$ tem a propriedade $(\mathrm{CLL})$ em $J \times\left(\Omega \times \Omega^{\prime}\right) \times V$ para todo $\gamma=\left(\gamma_{0}, \gamma_{1}, \ldots, \gamma_{3 n}\right) \in \mathbb{I}^{3 n+1}$ com $\gamma_{0}=\gamma_{j}=0<|\gamma|=1$ para todo $2 n+1 \leq j \leq 3 n$ e $1 \leq i \leq 2 n$, e assim, por 3.2.17, concluímos que $(X, P)=(\bar{X}, \bar{P})$ e portanto $\operatorname{card} \mathcal{S}\left(I, \Omega, \Omega^{\prime}, H, f, J, W\right)=1 . / /$ 
3.3.4 Teorema. Sejam $\Omega^{\prime}$ um aberto de $\mathbb{R}^{n}, I$ e $J$ intervalos abertos de $\mathbb{R}$ com $0 \in$ $J \subset I, H \in \mathcal{G}\left(I \times \mathbb{R}^{n} \times \Omega^{\prime} ; \mathbb{R}\right), \widehat{H}$ um representante de $H$ e $f \in \mathcal{G}\left(\mathbb{R}^{n} ; \mathbb{R}\right)$ tais que $\mathcal{S}\left(I, \mathbb{R}^{n}, \Omega^{\prime}, H, f, J, J \times \mathbb{R}^{n}\right) \neq \emptyset$. Denotando por $(t, x, p)=\left(t, x_{1}, \ldots, x_{n}, p_{1}, \ldots, p_{n}\right)$ um ponto genérico de $I \times \mathbb{R}^{n} \times \Omega^{\prime}$ tem-se que, se

(i) $\frac{\partial \widehat{H}}{\partial p} \quad$ é uma aplicação limitada em $I \times \mathbb{R}^{n} \times \Omega^{\prime}$;

(ii) $\frac{\partial^{2} \widehat{H}}{\partial x_{j} \partial p_{i}}, \frac{\partial^{2} \widehat{H}}{\partial x_{j} \partial x_{i}}$ e $\frac{\partial^{2} \widehat{H}}{\partial p_{j} \partial p_{i}} \quad$ têm a propriedade (CLL) em $I \times \mathbb{R}^{n} \times \Omega^{\prime}$, para todo $1 \leq i, j \leq n$;

(iii) $\frac{\partial \widehat{H}}{\partial x} \quad e \frac{\partial \widehat{H}}{\partial p}$ têm a propriedade (LLL) em $\left(I, \mathbb{R}^{n} \times \Omega^{\prime}\right)$,

se $u \in \mathcal{G}\left(J \times \mathbb{R}^{n} ; \mathbb{R}\right)$ é como em $(\mathbf{A})$ e se existe uma função $v \in \mathcal{G}\left(J \times \mathbb{R}^{n} ; \mathbb{R}\right)$ satisfazendo

(iv) $v$ é uma solução para o problema $\mathbf{H J}$ em $\mathcal{G}\left(J \times \mathbb{R}^{n} ; \mathbb{R}\right)$;

(v) existem um representante $\hat{v}$ de $v$ e $\tau \in] \cap, 1]$ tais que

$$
\left(\frac{\partial \widehat{v}}{\partial x_{1}}, \ldots, \frac{\partial \widehat{v}}{\partial x_{n}}\right)(] 0, \tau\left[\times J \times \mathbb{R}^{n}\right) \subset \Omega^{\prime}
$$

onde $(t, x)=\left(t, x_{1}, \ldots, x_{n}\right)$ denota um ponto genérico de $J \times \mathbb{R}^{n}$;

(vi) $\partial^{\alpha} v \in \mathcal{G}_{*}\left(J \times \mathbb{R}^{n} ; \mathbb{R}\right)$, para todo $\alpha=\left(\alpha_{0}, \alpha_{1}, \ldots, \alpha_{n}\right) \in \mathbb{N}^{n+1}$ com $\alpha_{0}=0<$ $|\alpha|=2$,

então $u=v$.

Demonstração. Denotaremos por $(t, x, p, r)=\left(t, x_{1}, \ldots, x_{n}, p_{1}, \ldots, p_{n}, r_{1}, \ldots, r_{n}\right)$ um ponto genérico de $\mathbb{R}^{3 n+1}$ e por $(t, x, r)=\left(t, x_{1}, \ldots, x_{n}, r_{1}, \ldots, r_{n}\right)$ ou $(t, x, p)=\left(t, x_{1}, \ldots, x_{n}, p_{1}, \ldots, p_{n}\right)$ um ponto genérico de $\mathbb{R}^{2 n+1}$.

Sejam $\tau$ e $\hat{v} \operatorname{com}$ em (v) e $\hat{\varphi}$ a aplicação definida em $] 0,1] \times J \times \mathbb{R}^{n} \times \mathbb{R}^{n}$ por $\hat{\varphi}(\varepsilon, t, x, r)=\frac{\partial \widehat{H}}{\partial p}\left(\varepsilon, t, x, \frac{\partial \widehat{v}}{\partial x_{1}}(\varepsilon, t, x), \ldots, \frac{\partial \widehat{v}}{\partial x_{n}}(\varepsilon, t, x)\right)$ se $\left.(\varepsilon, t, x, r) \in\right] 0, \tau\left[\times J \times \mathbb{R}^{n} \times \mathbb{R}^{n}\right.$ $\widehat{\varphi}$ é constante em $[\tau, 1] \times J \times \mathbb{R}^{n} \times \mathbb{R}^{n}$. 
Como $v$ é uma solução para o problema $\mathbf{H J}$ em $\mathcal{G}\left(J \times \mathbb{R}^{n} ; \mathbb{R}\right)$ temos que $\left(\frac{\partial v}{\partial x_{1}}, \ldots, \frac{\partial v}{\partial x_{n}}\right)$ pertence a $\mathcal{G}_{*}\left(J \times \mathbb{R}^{n} ; \Omega^{\prime}\right)$, e portanto a aplicação $\hat{\varphi} \in \mathcal{E}_{M}\left[J \times \mathbb{R}^{n} \times \mathbb{R}^{n} ; \mathbb{R}^{n}\right]$ e, por (i), temos que

$$
\hat{\varphi} \text { é limitada em } J \times \mathbb{R}^{n} \times \mathbb{R}^{n} \text {. }
$$

Seja $\varphi$ a classe de $\hat{\varphi}$ em $\mathcal{G}\left(J \times \mathbb{R}^{n} \times \mathbb{R}^{n} ; \mathbb{R}^{n}\right)$ que, por $(1)$, pertence a $\mathcal{G}_{*}\left(J \times \mathbb{R}^{n} \times\right.$ $\left.\mathbb{R}^{n} ; \mathbb{R}^{n}\right)$.

Fixemos $t \in J$ e consideremos em $\mathcal{G}\left(J \times \mathbb{R}^{n} ; \mathbb{R}^{n}\right)$ a equação de incógnita $\tilde{X}$

$$
\frac{\partial \tilde{X}}{\partial s}=\varphi \circ\left(\pi, \tilde{X}, \pi_{1}, \ldots, \pi_{n}\right)=\frac{\partial H}{\partial p} \circ\left(\pi, \tilde{X}, \frac{\partial v}{\partial x_{1}} \circ(\pi, \tilde{X}), \ldots, \frac{\partial v}{\partial x_{n}} \circ(\pi, \tilde{X})\right)
$$

com a condição

$$
\left.\tilde{X}\right|_{\{t\} \times R^{n}}=\left.X\right|_{\{t\} \times R^{n}} .
$$

onde $X$ é tal que $(X, P) \in \mathcal{S}\left(I, \mathbb{R}^{n}, \Omega^{\prime}, H, f, J, J \times \mathbb{R}^{n}\right)$.

Veremos, a seguir, que (2) com a condição (3) admite uma solução $\tilde{X}_{t} \in \mathcal{G}_{*}\left(I_{a_{t}}(t) \times\right.$ $\left.\mathbb{R}^{n} ; \mathbb{R}^{n}\right)$, para algum $a_{t}>0$. Para isto usaremos $3.2 .14\left(\operatorname{com} \varphi,\left.X\right|_{\{t\} \times R^{n}}\right.$ e $\mathbb{R}^{n}$ no lugar de $f, g$ e $\left.\Omega^{\prime}\right)$.

Como $(X, P) \in \mathcal{S}\left(I, \mathbb{R}^{n}, \Omega^{\prime}, H, f, J, J \times \mathbb{R}^{n}\right)$ temos que $X \in \mathcal{G}_{*}\left(J \times \mathbb{R}^{n} ; \mathbb{R}^{n}\right)$ e portanto

$$
\left.X\right|_{\{t\} \times R^{n}} \in \mathcal{G}_{*}\left(\mathbb{R}^{n} ; \mathbb{R}^{n}\right)
$$

e como vale (1) basta, para verificar as hipóteses de 3.2.14, provar que

$\frac{\partial \hat{\varphi}_{i}}{\partial x_{j}}$ tem a propriedade (CLL) em $J \times \mathbb{R}^{n} \times \mathbb{R}^{n}$, para todo $1 \leq i, j \leq n$.

Para $1 \leq i, j \leq n$ sejam $\hat{l}_{i, j}, \widehat{\psi}_{i, j}$ e $\widehat{\Phi}_{i, j}$ funções moderadas definidas em $\left.] 0,1\right] \times$ $J \times \mathbb{R}^{n} \times \mathbb{R}^{n}$ tais que, em $] 0, \tau\left[\times J \times \mathbb{R}^{n} \times \mathbb{R}^{n}\right.$ valem as seguintes igualdades:

$$
\begin{aligned}
& \hat{l}_{i, j}(\varepsilon, t, x, r)=\frac{\partial^{2} \widehat{H}}{\partial p_{j} \partial p_{i}}\left(\varepsilon, t, x, \frac{\partial \widehat{v}}{\partial x_{1}}(\varepsilon, t, x), \ldots, \frac{\partial \widehat{v}}{\partial x_{n}}(\varepsilon, t, x)\right) ; \\
& \widehat{\psi}_{i, j}(\varepsilon, t, x, r)=\frac{\partial^{2} \widehat{H}}{\partial x_{j} \partial p_{i}}\left(\varepsilon, t, x, \frac{\partial \widehat{v}}{\partial x_{1}}(\varepsilon, t, x), \ldots, \frac{\partial \widehat{v}}{\partial x_{n}}(\varepsilon, t, x)\right) ;
\end{aligned}
$$




$$
\widehat{\Phi}_{i, j}(\varepsilon, t, x, r)=\frac{\partial^{2} \widehat{v}}{\partial x_{j} \partial x_{i}}(\varepsilon, t, x)
$$

Portanto, para todo $1 \leq i, j \leq n$ e $(\varepsilon, t, x, r) \in] 0, \tau\left[\times J \times \mathbb{R}^{n} \times \mathbb{R}^{n}\right.$ temos que

$$
\frac{\partial \widehat{\varphi}_{i}}{\partial x_{j}}(\varepsilon, t, x, r)=\hat{\psi}_{i, j}(\varepsilon, t, x, r)+\sum_{k=1}^{n} \hat{l}_{i, k}(\varepsilon, t, x, r) \widehat{\Phi}_{k, j}(\varepsilon, t, x, r) .
$$

Sejam $J_{1} \subset \subset J, K_{1} \subset \subset \mathbb{R}^{n}$ e $K_{2} \subset \subset \mathbb{R}^{n} . \operatorname{Como}\left(\frac{\partial \widehat{v}}{\partial x_{1}}, \ldots, \frac{\partial \widehat{v}}{\partial x_{n}}\right) \in \mathcal{G}_{*}\left(J \times \mathbb{R}^{n} ; \Omega^{\prime}\right)$ existem $K^{\prime} \subset \subset \Omega^{\prime}$ e $\left.\eta \in\right] 0, \tau[$ tais que

$$
\left(\frac{\partial \widehat{v}}{\partial x_{1}}, \ldots, \frac{\partial \widehat{v}}{\partial x_{n}}\right)(] 0, \eta\left[\times J_{1} \times K_{1}\right) \subset K^{\prime} \subset \subset \Omega^{\prime},
$$

e assim usando (6), (7), (ii), (vi) e 3.1 .2 obtemos (5).

Portanto, por 3.2.14, existem $a_{t}>0$ com $\overline{I_{a_{t}}(t)} \subset J$ e $\tilde{X}_{t} \in \mathcal{G}_{*}\left(I_{a_{t}}(t) \times \mathbb{R}^{n} ; \mathbb{R}^{n}\right)$ que é solução de (2) e (3).

Como $J \times \mathbb{R}^{n}=\cup_{t \in J} I_{a_{t}}(t) \times \mathbb{R}^{n}$ existe, por 1.1.20.III, uma aplicação $\tilde{X} \in \mathcal{G}_{*}(J \times$ $\left.\mathbb{R}^{n} ; \mathbb{R}^{n}\right)$ tal que

$$
\left.\tilde{X}\right|_{I_{a_{t}}(t) \times R^{n}}=\tilde{X}_{t}, \quad \text { para todo } t \in J
$$

e portanto

$$
\begin{aligned}
& \tilde{X} \text { é solução de }(2) \text { em } \mathcal{G}\left(J \times \mathbb{R}^{n} ; \mathbb{R}^{n}\right) ; \\
& \left.\tilde{X}\right|_{\{0\} \times R^{n}}=\left.\tilde{X}_{0}\right|_{\{0\} \times R^{n}}=\left.X\right|_{\{0\} \times R^{n}}=1_{R^{n}} .
\end{aligned}
$$

Seja $\widehat{\Psi}$ a aplicação moderada definida em $] 0,1] \times J \times \mathbb{R}^{n} \times \Omega^{\prime} \times \mathbb{R}^{n}$ por

$$
\widehat{\Psi}(\varepsilon, s, x, p, r)=\left(\frac{\partial \widehat{H}}{\partial p}(\varepsilon, t, x, p),-\frac{\partial \widehat{H}}{\partial x}(\varepsilon, t, x, p)\right)
$$

e $\Psi$ a classe de $\widehat{\Psi}$ em $\mathcal{G}\left(J \times \mathbb{R}^{n} \times \Omega^{\prime} \times \mathbb{R}^{n} ; \mathbb{R}^{2 n}\right)$.

Seja $\tilde{P}=\left(\frac{\partial v}{\partial x_{1}} \circ(\pi, \tilde{X}), \ldots, \frac{\partial v}{\partial x_{n}} \circ(\pi, \tilde{X})\right)$. Provaremos, a seguir, que $(\tilde{X}, \tilde{P})$ é uma solução, em $\mathcal{G}\left(J \times \mathbb{R}^{n} ; \mathbb{R}^{2 n}\right)$, do sistema de incógnitas $\bar{X}$ e $\bar{P}$

$$
\begin{aligned}
& \frac{\partial \bar{X}}{\partial s}=\frac{\partial H}{\partial p} \circ(\pi, \bar{X}, \bar{P}) \\
& \frac{\partial \bar{P}}{\partial s}=-\frac{\partial H}{\partial x} \circ(\pi, \bar{X}, \bar{P}),
\end{aligned}
$$

com a condição 


$$
\left.\bar{X}\right|_{\{0\} \times R^{n}}=1_{R^{n}} \text { e }\left.\quad \bar{P}\right|_{\{0\} \times R^{n}}=\nabla f,
$$

isto é, valem (13) e

$$
\left(\frac{\partial \bar{X}}{\partial s}, \frac{\partial \bar{P}}{\partial s}\right)=\Psi \circ\left(\pi, \bar{X}, \bar{P}, \pi_{1}, \ldots, \pi_{n}\right) .
$$

Como $\tilde{X}$ é solução de (2) temos que (11) é verdadeira para $(\tilde{X}, \tilde{P})$.

Notemos que

$$
\begin{aligned}
\frac{\partial \tilde{P}_{i}}{\partial s} & =\frac{\partial}{\partial s}\left(\frac{\partial v}{\partial x_{i}} \circ(\pi, \tilde{X})\right) \\
& =\frac{\partial^{2} v}{\partial x_{i} \partial t} \circ(\pi, \tilde{X})+\sum_{j=1}^{n}\left(\frac{\partial^{2} v}{\partial x_{j} \partial x_{i}} \circ(\pi, \tilde{X})\right) \frac{\partial \tilde{X}_{j}}{\partial s}
\end{aligned}
$$

e como $v$ é uma solução para o problema $\mathbf{H J}$ em $\mathcal{G}\left(J \times \mathbb{R}^{n} ; \mathbb{R}\right)$ temos que

$$
\frac{\partial v}{\partial t}=-H \circ\left(\pi, \pi_{1}, \ldots, \pi_{n}, \frac{\partial v}{\partial x_{1}}, \ldots, \frac{\partial v}{\partial x_{n}}\right),
$$

e portanto

$$
\begin{aligned}
\frac{\partial \tilde{P}_{i}}{\partial s}= & -\frac{\partial H}{\partial x_{i}} \circ\left(\pi, \tilde{X}, \frac{\partial v}{\partial x_{1}} \circ(\pi, \tilde{X}), \ldots, \frac{\partial v}{\partial x_{n}} \circ(\pi, \tilde{X})\right)+ \\
& -\sum_{j=1}^{n} \frac{\partial H}{\partial p_{j}} \circ\left(\pi, \tilde{X}, \frac{\partial v}{\partial x_{1}} \circ(\pi, \tilde{X}), \ldots, \frac{\partial v}{\partial x_{n}} \circ(\pi, \tilde{X})\right) \frac{\partial^{2} v}{\partial x_{i} \partial x_{j}} \circ(\pi, \tilde{X})+ \\
& +\sum_{j=1}^{n}\left(\frac{\partial^{2} v}{\partial x_{j} \partial x_{i}} \circ(\pi, \tilde{X})\right) \frac{\partial \tilde{X}_{j}}{\partial s} \\
= & -\frac{\partial H}{\partial x_{i}} \circ(\pi, \tilde{X}, \tilde{P})-\sum_{j=1}^{n} \frac{\partial H}{\partial p_{j}} \circ(\pi, \tilde{X}, \tilde{P}) \frac{\partial^{2} v}{\partial x_{i} \partial x_{j}} \circ(\pi, \tilde{X})+ \\
& +\sum_{j=1}^{n}\left(\frac{\partial^{2} v}{\partial x_{j} \partial x_{i}} \circ(\pi, \tilde{X})\right) \frac{\partial \tilde{X}_{j}}{\partial s},
\end{aligned}
$$

e como vale (2) para $\tilde{X}$ temos que

$$
\frac{\partial \tilde{P}_{i}}{\partial s}=-\frac{\partial H}{\partial x_{i}} \circ(\pi, \tilde{X}, \tilde{P}), \text { para todo } 1 \leq i \leq n .
$$

Usando 1.1.25 temos que

$$
\begin{aligned}
\left.\tilde{P}\right|_{\{0\} \times R^{n}} & =\left.\left(\frac{\partial v}{\partial x_{1}} \circ(\pi, \tilde{X}), \ldots, \frac{\partial v}{\partial x_{n}} \circ(\pi, \tilde{X})\right)\right|_{\{0\} \times R^{n}} \\
& =\left(\frac{\partial v}{\partial x_{1}} \circ\left(0,\left.\tilde{X}\right|_{\{0\} \times R^{n}}\right), \ldots, \frac{\partial v}{\partial x_{n}} \circ\left(0,\left.\tilde{X}\right|_{\{0\} \times R^{n}}\right)\right),
\end{aligned}
$$


e por (10) e 1.1.27 temos que

$$
\begin{aligned}
\left.\tilde{P}\right|_{\{0\} \times R^{n}} & =\left(\frac{\partial v}{\partial x_{1}} \circ\left(0,1_{R^{n}}\right), \ldots, \frac{\partial v}{\partial x_{n}} \circ\left(0,1_{R^{n}}\right)\right) \\
& =\left.\left(\frac{\partial v}{\partial x_{1}}, \ldots, \frac{\partial v}{\partial x_{n}}\right)\right|_{\{0\} \times R^{n}}
\end{aligned}
$$

e como $\left.v\right|_{\{0\} \times R^{n}}=f$ temos que

$$
\left.\tilde{P}\right|_{\{0\} \times R^{n}}=\nabla f
$$

Portanto $(\tilde{X}, \tilde{P})$ é uma solução de (14) com a condição (13).

Como $(X, P) \in \mathcal{S}\left(I, \mathbb{R}^{n}, \Omega^{\prime}, H, f, J, J \times \mathbb{R}^{n}\right)$ temos que $(X, P)$ satisfaz (13) e (14) e como, por (ii) e (iii), $\Psi$ tem a propriedade (LLL) em $\left(J, \mathbb{R}^{n} \times \Omega^{\prime}, \mathbb{R}^{n}\right)$ e $\frac{\partial \Psi_{i}}{\partial x_{j}}$ e $\frac{\partial \Psi_{i}}{\partial p_{j}}$ têm a propriedade (CLL) em $J \times\left(\mathbb{R}^{n} \times \Omega^{\prime}\right) \times \mathbb{R}^{n}$ para todo $1 \leq i \leq 2 n$ e $1 \leq j \leq n$, temos, por 3.2.17, que $(\tilde{X}, \tilde{P})=(X, P)$, e assim, usando $(\mathbf{B})$ (definido no início desta seção), concluímos que

$$
\left(\frac{\partial u}{\partial x_{1}}, \ldots, \frac{\partial u}{\partial x_{n}}\right) \circ(\pi, X)=P=\tilde{P}=\left(\frac{\partial v}{\partial x_{1}}, \ldots, \frac{\partial v}{\partial x_{n}}\right) \circ(\pi, \tilde{X})=\left(\frac{\partial v}{\partial x_{1}}, \ldots, \frac{\partial v}{\partial x_{n}}\right) \circ(\pi, X),
$$

e como $(\pi, X) \in \mathcal{G}_{*}\left(J \times \mathbb{R}^{n} ; J \times \mathbb{R}^{n}\right)$ é uma aplicação inversível (2.1.1.iv) temos que

$$
\left(\frac{\partial u}{\partial x_{1}}, \ldots, \frac{\partial u}{\partial x_{n}}\right)=\left(\frac{\partial v}{\partial x_{1}}, \ldots, \frac{\partial v}{\partial x_{n}}\right) \text { em } \mathcal{G}\left(J \times \mathbb{R}^{n} ; \mathbb{R}^{n}\right)
$$

e assim, por (15), temos que

$$
\begin{aligned}
& \frac{\partial u}{\partial t}=-H \circ\left(\pi, \pi_{1}, \ldots, \pi_{n}, \frac{\partial u}{\partial x_{1}}, \ldots, \frac{\partial u}{\partial x_{n}}\right)=-H \circ\left(\pi, \pi_{1}, \ldots, \pi_{n}, \frac{\partial v}{\partial x_{1}}, \ldots, \frac{\partial v}{\partial x_{n}}\right)=\frac{\partial v}{\partial t} . \\
& \text { Como } \frac{\partial u}{\partial t}=\frac{\partial v}{\partial t} \text { em } \mathcal{G}\left(J \times \mathbb{R}^{n} ; \mathbb{R}\right) \mathrm{e}
\end{aligned}
$$

$$
\left.(u-v)\right|_{\{0\} \times R^{n}}=\left.u\right|_{\{0\} \times R^{n}}-\left.v\right|_{\{0\} \times R^{n}}=f-f=0
$$

temos, por 1.1 .15 , que $u=v$ em $\mathcal{G}\left(J \times \mathbb{R}^{n} ; \mathbb{R}\right)$.//

Completamos o resultado acima com os seguintes exemplos: 
3.3.5 Exemplo. Sejam $b>0, \hat{f} \in \mathcal{E}_{M}\left[\mathbb{R}^{n} ; \mathbb{R}\right]$ e $\hat{h} \in \mathcal{E}_{M}\left[I_{b} \times \mathbb{R}^{n} ; \mathbb{R}\right]$ como em 2.1.6, $\mu=\left(\mu_{1}, \ldots, \mu_{n}\right)$ como em 2.1.5, $\widehat{H}$ a função moderada definida em $\left.] 0,1\right] \times I_{b} \times \mathbb{R}^{n} \times \mathbb{R}^{n}$ por

$$
\widehat{H}\left(\varepsilon, t, x_{1}, \ldots, x_{n}, p_{1}, \ldots, p_{n}\right)=\widehat{h}\left(\varepsilon, t, p_{1}, \ldots, p_{n}\right)+\sum_{i=1}^{n} \mu_{i}(\varepsilon) x_{i}
$$

e $H$ a classe de $\widehat{H}$ em $\mathcal{G}\left(I_{b} \times \mathbb{R}^{n} \times \mathbb{R}^{n} ; \mathbb{R}\right)$. Então existe $a>0$ tal que $\overline{I_{a}} \subset I_{b}$ e existe uma única função $v \in \mathcal{G}\left(I_{a} \times \mathbb{R}^{n} ; \mathbb{R}\right)$ que satisfaz de 3.3.4.iv até 3.3.4.vi.

De fato, usando 3.1.6 e o Teorema do Valor Médio prova-se que vale 3.3.4.iii, e assim o resultado segue de 2.1.5, 2.1.6 e 3.3.4.

3.3.6 Exemplo. Sejam $1 \leq j \leq n, \mu_{j}, \nu_{j}$ e $\tilde{\nu}_{j}$ funções definidas em $\left.] 0,1\right]$ e com valores em $\mathbb{R}$ e $\psi_{j}, \Psi_{j}$ e $\Phi_{j}$ funçôes pertencentes a $\mathcal{C}^{\infty}(\mathbb{R} ; \mathbb{R})$ tais que $\mu_{j}, \nu_{j}, \tilde{\nu}_{j}, \psi_{j}, \Psi_{j}$ e $\Phi_{j}$ são funções limitadas, $\left.\left.\inf \left\{\left|\tilde{\nu}_{j}(\varepsilon)\right|: \varepsilon \in\right] 0,1\right]\right\}>0$ e $\Phi_{j} \in L_{1}(\mathbb{R})$. Se $\hat{f}$ é a função moderada definida em $] 0,1] \times \mathbb{R}^{n}$ por

$$
\widehat{f}\left(\varepsilon, r_{1}, \ldots, r_{n}\right)=\sum_{j=1}^{n} \int_{0}^{r_{j}}\left(\int_{0}^{\lambda} \Psi_{j}\left(\nu_{j}(\varepsilon) x\right) d x\right) d \lambda,
$$

$\widehat{H}$ é a função moderada definida em $] 0,1] \times \mathbb{R} \times \mathbb{R}^{n} \times \mathbb{R}^{n}$ por

$$
\widehat{H}\left(\varepsilon, t, x_{1}, \ldots, x_{n}, p_{1}, \ldots, p_{n}\right)=\sum_{j=1}^{n} \psi_{j}\left(\mu_{j}(\varepsilon) t\right) \int_{0}^{p_{j}}\left(\int_{0}^{\lambda} \Phi_{j}\left(\tilde{\nu}_{j}(\varepsilon) x\right) d x\right) d \lambda
$$

e $H$ é a classe de $\widehat{H}$ em $\mathcal{G}\left(\mathbb{R} \times \mathbb{R}^{n} \times \mathbb{R}^{n} ; \mathbb{R}\right)$, então existe a>0 e existe uma única função $v \in \mathcal{G}\left(I_{a} \times \mathbb{R}^{n} ; \mathbb{R}\right)$ que satisfaz 3.3.4.iv até 3.3.4.vi.

(Análoga à de 3.3.5 substituindo, em 3.3.5, 2.1.5 e 2.1.6 por 2.1.7 e 2.1.8 respectivamente.)

No próximo resultado iremos fazer $\Omega^{\prime}=\mathbb{R}^{n}$ e substituiremos 3.3.4.i por 3.3.7.i .

3.3.7 Teorema. Sejam $I$ e $J$ intervalos abertos de $\mathbb{R}$ com $0 \in J \subset I, H \in \mathcal{G}\left(I \times \mathbb{R}^{n} \times\right.$ $\left.\mathbb{R}^{n} ; \mathbb{R}\right), \widehat{H}$ um representante de $H$ e $f \in \mathcal{G}\left(\mathbb{R}^{n} ; \mathbb{R}\right)$ tais que $\mathcal{S}\left(I, \mathbb{R}^{n}, \mathbb{R}^{n}, H, f, J, J \times\right.$ $\left.\mathbb{R}^{n}\right) \neq \emptyset$. Denotando por $B_{r}$ a bola aberta de centro 0 e raio $r$ em $\mathbb{R}^{n}$ e por $(t, x, p)$ um ponto genérico de $I \times \mathbb{R}^{n} \times \mathbb{R}^{n}$ tem-se que, se 
(i) existem $M>0$ e $\tau \in] 0,1]$ tais que $\frac{\partial \widehat{H}}{\partial p}(] 0, \tau\left[\times I \times \overline{B_{r}} \times \overline{B_{r}}\right) \subset \overline{B_{M r}}$, para todo $r>0$;

(ii) $\frac{\partial^{2} \widehat{H}}{\partial x_{j} \partial p_{i}}, \frac{\partial^{2} \widehat{H}}{\partial x_{j} \partial x_{i}}$ e $\frac{\partial^{2} \widehat{H}}{\partial p_{j} \partial p_{i}}$ têm a propriedade (CLL) em $I \times \mathbb{R}^{n} \times \mathbb{R}^{n}$, para todo $1 \leq i, j \leq n$;

(iii) $\frac{\partial \widehat{H}}{\partial x} \quad e \frac{\partial \widehat{H}}{\partial F}$ têm a propriedade (LLL) em $\left(I, \mathbb{R}^{n} \times \mathbb{R}^{n}\right)$,

e se $u \in \mathcal{G}\left(J \times \mathbb{R}^{n} ; \mathbb{R}\right)$ é como em $(\mathbf{A})$ e se existe uma função $v \in \mathcal{G}\left(J \times \mathbb{R}^{n} ; \mathbb{R}\right)$ satisfazendo

(iv) $v$ é uma solução para o problema $\mathbf{H J}$ em $\mathcal{G}\left(J \times \mathbb{R}^{n} ; \mathbb{R}\right)$;

(v) existem um representante $\widehat{v}$ de $v, M_{1}>0$ e $\left.\left.\tau_{1} \in\right] 0,1\right]$ tais que para todo $r>0$

e $\alpha=\left(\alpha_{0}, \alpha_{1}, \ldots, \alpha_{n}\right) \in \mathbb{N}^{n+1}$ com $\alpha_{0}=0<|\alpha|=1$, tem-se que

$$
\partial^{\alpha} \widehat{v}(] 0, \tau_{1}\left[\times J \times \overline{B_{r}}\right) \subset \overline{B_{r M_{1}}}
$$

(vi) $\partial^{\alpha} v \in \mathcal{G}_{*}\left(J \times \mathbb{R}^{n} ; \mathbb{R}\right)$, para todo $\alpha=\left(\alpha_{0}, \alpha_{1}, \ldots, \alpha_{n}\right) \in \mathbb{N}^{n+1}$ com $\alpha_{0}=0<$ $|\alpha|=2$,

então $u=v$.

Demonstração. Análoga à de 3.3.4 substituindo, em 3.3.4 , 3.2.14 por 3.2.15 e observando que, por (i) e (v),

$$
\begin{aligned}
\hat{\varphi}(] 0, \tau_{2}\left[\times J \times \overline{B_{r}} \times \overline{B_{r}}\right) & \subset \frac{\partial \widehat{H}}{\partial p}(] 0, \tau_{2}\left[\times J \times \overline{B_{r}} \times \overline{B_{r M_{2}}}\right) \\
& \subset \frac{\partial \widehat{H}}{\partial p}(] 0, \tau_{2}\left[\times J \times \overline{B_{r M_{2}}} \times \overline{B_{r M_{2}}}\right) \subset \overline{B_{r M M_{2}}},
\end{aligned}
$$

para todo $r>0$, sendo $M_{2}=\max \left\{1, M_{1}\right\}$ e $\tau_{2}=\min \left\{\tau, \tau_{1}\right\} \cdot / /$

Completamos o resultado acima com o seguinte exemplo: 
3.3.8 Exemplo. Sejam $1 \leq j \leq n, \mu_{j}$ e $\nu_{j}$ funções definidas em $\left.] 0,1\right]$ e com valores em $\mathbb{R}$ tais que $\left.\left.\left.\left.\left.\left.\mu_{j}(] 0,1\right]\right) \cup \nu_{j}(] 0,1\right]\right) \subset\right] 0,1\right], \widehat{H}$ a aplicação moderada definida em $\left.\left.] 0,1\right] \times\right]$ $\frac{1}{8}, \infty\left[\times \mathbb{R}^{n} \times \mathbb{R}^{n}\right.$ por

$$
\widehat{H}\left(\varepsilon, t, x_{1}, \ldots, x_{n}, p_{1}, \ldots, p_{n}\right)=\sum_{j=1}^{n} \mu_{j}(\varepsilon) p_{j}^{2},
$$

$\hat{f}$ a função moderada definida em $] 0,1] \times \mathbb{R}^{n}$ por

$$
\widehat{f}\left(\varepsilon, r_{1}, \ldots, r_{n}\right)=\sum_{j=1}^{n} \nu_{j}(\varepsilon) r_{j}^{2}
$$

$H$ a classe de $\widehat{H}$ em $\mathcal{G}(]-\frac{1}{8}, \infty\left[\times \mathbb{R}^{n} \times \mathbb{R}^{n} ; \mathbb{R}\right)$ e f a classe de $\widehat{f}$ em $\mathcal{G}\left(\mathbb{R}^{n} ; \mathbb{R}\right)$, então existe uma única solução para o prob̄lema $\mathbf{H J}$ em $\mathcal{G}(]-\frac{1}{8}, \infty\left[\times \mathbb{R}^{n} ; \mathbb{R}\right)$ que satisfaz $3.3 .7 . \mathrm{v}$ e 3.3.7.vi.

De fato, basta observar que, por 2.1.3, podemos determinar uma solução para o problema HJ em $\mathcal{G}(]-\frac{1}{8}, \infty\left[\times \mathbb{R}^{n} ; \mathbb{R}\right)$ que tem como representante a função

$$
\widehat{v}\left(\varepsilon, t, x_{1}, \ldots, x_{n}\right)=\sum_{j=1}^{n} \frac{\nu_{j}(\varepsilon) x_{j}^{2}+4 t \mu_{j}(\varepsilon)\left(\nu_{j}(\varepsilon)\right)^{2} x_{j}^{2}}{\left(1+4 \mu_{j}(\varepsilon) \nu_{j}(\varepsilon) t\right)^{2}},
$$

e assim, por 3.3.7, ela é a única solução que satisfaz 3.3.7.v e 3.3.7.vi.)

Com o auxílio de 3.3 .1 e 3.3.4 obtemos:

3.3.9 Teorema. Sejam I um intervalo aberto de $\mathbb{R}$ com $0 \in I, f \in \mathcal{G}\left(\mathbb{R}^{n} ; \mathbb{R}\right), H \in$ $\mathcal{G}\left(I \times \mathbb{R}^{n} \times \mathbb{R}^{n} ; \mathbb{R}\right), \hat{f}$ um representante de $f$ e $\widehat{H}$ um representante de $H$. Denotando por $(t, x, p)$ um ponto genérico de $\mathbb{R}^{2 n+1}$ tem-se que, se

(i) as asserções 3.3.1.i até 3.3.1.iii estão satisfeitas;

(ii) $\frac{\partial \widehat{H}}{\partial x} \quad e \frac{\partial \widehat{H}}{\partial p}$ têm a propriedade (LLL) em $\left(I, \mathbb{R}^{n} \times \mathbb{R}^{n}\right)$,

então existe $a>0$ com $\overline{I_{a}} \subset I$ e existe uma única função $u \in \mathcal{G}\left(I_{a} \times \mathbb{R}^{n} ; \mathbb{R}\right)$ tal que

(I) u é uma solução para o problema $\mathbf{H J}$ em $\mathcal{G}\left(I_{a} \times \mathbb{R}^{n} ; \mathbb{R}\right)$; 
(II) $\partial^{\alpha} u \in \mathcal{G}_{*}\left(I_{a} \times \mathbb{R}^{n} ; \mathbb{R}\right)$, para todo $\alpha=\left(\alpha_{0}, \alpha_{1}, \ldots, \alpha_{n}\right) \in \mathbb{N}^{n+1}$ com $\alpha_{0}=0<$ $|\alpha|=2$. //

Completamos o resultado acima com o seguinte exemplo:

3.3.10 Exemplo. Sejam $b>0$ e $H$ e $f$ como em 3.3.2. Então existe $a>0$ tal que $\overline{I_{a}} \subset I$ e existe uma única função $v \in \mathcal{G}\left(I_{a} \times \mathbb{R}^{n} ; \mathbb{R}\right)$ que satisfaz 3.3.9.I e 3.3.9.II. (Basta usar 3.3.1 e 3.3.2 e observar que, por 3.1.6 e o Teorema do Valor Médio, a função $\widehat{H}$ satisfaz 3.3.9.ii.)

Dos principais resultados apresentados neste trabalho resulta que o conjunto dos pares $(H, f)$ tais que o problema $\mathbf{H J}$ correspondente tem solução é significativo. De modo mais preciso:

Sejam $I$ um intervalo aberto de $\mathbb{R}$ com $0 \in I, \Omega$ e $\Omega^{\prime}$ abertos de $\mathbb{R}^{n}$ e

\section{$\mathcal{A}$}

o subcontunto de $\mathcal{G}\left(I \times \Omega \times \Omega^{\prime} ; \mathbb{R}\right) \times \mathcal{G}(\Omega ; \mathbb{R})$ formado pelos elementos $(H, f)$ tais que existem $J$ um intervalo aberto de $\mathbb{R}$ com $0 \in J \subset I, W$ um aberto de $\mathbb{R}^{n+1}$ com $V=\left\{z \in \mathbb{R}^{n}:(0, z) \in W\right\} \neq \emptyset$ e $W \subset I \times \Omega$ satisfazendo $\mathcal{S}\left(I, \Omega, \Omega^{\prime}, H, f, J, W\right) \neq \emptyset$, isto é, o problema $\mathbf{H J}$ tem solução.

Vimos em 2.1.4 que $\mathcal{C}^{\infty}\left(I \times \Omega \times \Omega^{\prime} ; \mathbb{R}\right) \times \mathcal{C}^{\infty}(\Omega ; \mathbb{R}) \subset \mathcal{A}$.

No caso particular que $\Omega=\Omega^{\prime}=\mathbb{R}^{n}$ introduzimos os seguintes conjuntos:

$$
\begin{aligned}
& \mathcal{A}_{1}=\left\{(H, f) \in \mathcal{G}\left(I \times \mathbb{R}^{n} \times \mathbb{R}^{n} ; \mathbb{R}\right) \times \mathcal{G}\left(\mathbb{R}^{n} ; \mathbb{R}\right): H \text { e } f \text { são como em } 2.1 .5\right\} ; \\
& \mathcal{A}_{2}=\left\{(H, f) \in \mathcal{G}\left(I \times \mathbb{R}^{n} \times \mathbb{R}^{n} ; \mathbb{R}\right) \times \mathcal{G}\left(\mathbb{R}^{n} ; \mathbb{R}\right): H \text { e } f \text { são como em } 2.1 .7\right\} ; \\
& \mathcal{A}_{3}=\left\{(H, f) \in \mathcal{G}\left(I \times \mathbb{R}^{n} \times \mathbb{R}^{n} ; \mathbb{R}\right) \times \mathcal{G}\left(\mathbb{R}^{n} ; \mathbb{R}\right): H \text { e } f \text { são como em 3.3.1 }\right\} .
\end{aligned}
$$

Então $\mathcal{A}_{1}$ e $\mathcal{A}_{3}$ são $\mathbb{R}$-subespaços vetoriais não triviais de $\mathcal{G}\left(I \times \mathbb{R}^{n} \times \mathbb{R}^{n} ; \mathbb{R}\right) \times$ $\mathcal{G}\left(\mathbb{R}^{n} ; \mathbb{R}\right)$ e $\mathcal{A}_{2}$ contém o $\mathbb{R}$-subespaço vetorial de $\mathcal{G}\left(I \times \mathbb{R}^{n} \times \mathbb{R}^{n} ; \mathbb{R}\right) \times \mathcal{G}\left(\mathbb{R}^{n} ; \mathbb{R}\right)$ dado pelos pares $(H, f)$ tais que satisfazem 2.1.7 substituindo 2.1.7.ii por 
(ii') $\frac{\partial \widehat{f}_{i}}{\partial y}(\varepsilon, 0)=\frac{\partial \hat{h}_{i}}{\partial y}(\varepsilon, t, 0)=0$, para todo $\left.(\varepsilon, t) \in\right] 0, \tau[\times I$ e $1 \leq i \leq n ;$

e 2.1.7.iii por

(iii')existe $M>0$ tal que, para todo $(\varepsilon, t, y) \in] 0, \tau[\times I \times \mathbb{R}$ e $1 \leq i \leq n$ tem-se

$$
\max \left\{\left|\frac{\partial^{2} \widehat{h}_{i}}{\partial y^{2}}(\varepsilon, t, y)\right|,\left|\frac{\partial^{2} \widehat{f}_{i}}{\partial y^{2}}(\varepsilon, y)\right|\right\} \leq M
$$

Vimos em 2.1.5, 2.1.7 e 3.3.1 que para $\Omega=\Omega^{\prime}=\mathbb{R}^{n}$ temos $\mathcal{A}_{1} \cup \mathcal{A}_{2} \cup \mathcal{A}_{3} \subset \mathcal{A}$ (sendo $W=\mathbb{R}^{n}$ ) e apresentamos em 2.1.9 e 2.1.11 funções $H$ e $f$ para as quais o par $(H, f) \in \mathcal{A}\left(\right.$ para $\Omega^{\prime}=\mathbb{R}_{+}^{* n}$ e $\left.\Omega^{\prime}=\right] 0,1\left[{ }^{n}\right.$, respectivamente $)$. Portanto o conjunto $\mathcal{A}$ nos parece razoavelmente "grande".

Encerramos este trabalho fazendo as seguintes considerações:

Apresentamos no capítulo 2 um método para construir, sob certas hipóteses, uma solução para o problema $\mathbf{H J}$ e vimos na seção 3.3 que, sob certas condições, existe uma única solução que satisfaz 3.3.4.vi. Surgem naturalmente as seguintes questões que pretendemos estudar no futuro:

(1) será que a existência de soluções para o problema $\mathbf{H J}$ em $\mathcal{G}(W ; \mathbb{R})$ implica

$$
\mathcal{S}\left(I, \Omega, \Omega^{\prime}, H, f, J, W\right) \neq \emptyset ?
$$

(2) se u é uma solução como em 2.1 .3 e $f=\left(f_{1}, \ldots, f_{n}\right) \in \mathcal{G}(\Omega ; \mathbb{R})$ é tal que $\partial^{\alpha} f_{i} \in$ $\mathcal{G}_{*}(\Omega ; \mathbb{R})$, para todo $\alpha \in \mathbb{N}^{n}$ com $|\alpha|=2$ e $1 \leq i \leq n$, será que u satisfaz 3.3.4.vi ?;

(3) se u é uma solução para o problema $\mathbf{H J}$ e $f$ é como em (2), será que u satisfaz 3.3.4.vi?.

Até o momento não temos respostas para as questões acima. 


\section{Apêndice}

Aqui utilizaremos as notações anteriores.

Com o auxílio dos resultados de 3.2 podemos estudar, conforme sugestão da Banca Examinadora, um problema mais geral que o problema $\mathbf{H J}$, isto é:

Problema A: Dados quaisquer I intervalo aberto de $\mathbb{R}$ com $0 \in I, I^{\prime}$ um intervalo aberto de $\mathbb{R}, \Omega$ e $\Omega^{\prime}$ abertos de $\mathbb{R}^{n}, H \in \mathcal{G}\left(I \times \Omega \times I^{\prime} \times \Omega^{\prime} ; \mathbb{R}\right)$ e $f \in \mathcal{G}(\Omega ; \mathbb{R})$, determinar um aberto $W$ de $I \times \Omega$ com $V=\left\{z \in \mathbb{R}^{n}:(0, z) \in W\right\} \neq \emptyset$ e uma função $u \in \mathcal{G}(W ; \mathbb{R})$ satisfazendo:

(i) $\left(u, \frac{\partial u}{\partial x_{1}}, \ldots, \frac{\partial u}{\partial x_{n}}\right) \in \mathcal{G}_{*}\left(W ; I^{\prime} \times \Omega^{\prime}\right)$;

(ii) $\frac{\partial u}{\partial t}+H \circ\left(\pi, \pi_{1}, \ldots, \pi_{n}, u, \frac{\partial u}{\partial x_{1}}, \ldots, \frac{\partial u}{\partial x_{n}}\right)=0$ em $\mathcal{G}(W ; \mathbb{R})$;

(iii) $\left.u\right|_{\{0\} \times V}=\left.f\right|_{V}$ em $\mathcal{G}(V ; \mathbb{R})$.

Dizemos que $u \in \mathcal{G}(W ; \mathbb{R})$ é uma solução para o problema $\mathbf{A}$ em $\mathcal{G}(W ; \mathbb{R})$ se, e somente se, $u$ satisfaz (i), (ii) e (iii).

O problema $\mathbf{H J}$ é um caso particular do problema $\mathbf{A}$.

Neste apêndice apresentaremos alguns resultados um pouco mais gerais que os das seções 2.1 e 3.3 que foram obtidos através da análise das provas desses e da utilização de 3.2. Por esta razão para obter as provas dos resultados aqui expostos iremos somente indicar as alterações que deverão ser feitas. 
A menos de menção em contrário denotaremos por $(t, x, y, p)=\left(t, x_{1}, \ldots, x_{n}, y, p_{1}, \ldots, p_{n}\right)$ um ponto genérico de $\mathbb{R}^{2 n+2}$.

A.1 Definição. Sejam I um intervalo aberto de $\mathbb{R}$ com $0 \in I$, I' um intervalo aberto de $\mathbb{R}, \Omega$ e $\Omega^{\prime}$ abertos de $\mathbb{R}^{n}, H \in \mathcal{G}\left(I \times \Omega \times I^{\prime} \times \Omega^{\prime} ; \mathbb{R}\right)$ e $f \in \mathcal{G}(\Omega ; \mathbb{R})$. Se $J$ é um intervalo aberto de $\mathbb{R}$ com $0 \in J \subset I, W$ um aberto de $\mathbb{R}^{n+1}$ com $V=\left\{z \in \mathbb{R}^{n}:(0, z) \in W\right\} \neq \emptyset$ e $W^{\prime} \subset I \times \Omega$, então denotaremos por

$$
\mathcal{S}_{1}\left(I, \Omega, I^{\prime}, \Omega^{\prime}, H, f, J, W\right)
$$

o conjunto das ternas $(X, U, P)$ para as quais são verdadeiras as seguintes asserções:

(i) $X \in \mathcal{G}_{*}(J \times V ; \Omega), \quad U \in \mathcal{G}_{*}\left(J \times V ; I^{\prime}\right) \quad$ e $P \in \mathcal{G}_{*}\left(J \times V ; \Omega^{\prime}\right)$;

(ii) $(X, U, P)$ é uma solução do sistema:

$$
\begin{aligned}
& \frac{\partial X}{\partial s}=\frac{\partial H}{\partial p} \circ(\pi, X, U, P) \quad \text { em } \mathcal{G}\left(J \times V ; \mathbb{R}^{n}\right) \\
& \frac{\partial P}{\partial s}=-\frac{\partial H}{\partial x} \circ(\pi, X, U, P)-P \frac{\partial H}{\partial y} \circ(\pi, X, U, P) \quad \text { em } \mathcal{G}\left(J \times V ; \mathbb{R}^{n}\right) \\
& \frac{\partial U}{\partial s}=-H \circ(\pi, X, U, P)+\sum_{j=1}^{n} \frac{\partial H}{\partial p_{j}} \circ(\pi, X, U, P) P_{j} \quad \text { em } \mathcal{G}(J \times V ; \mathbb{R}),
\end{aligned}
$$

sendo $P=\left(P_{1}, \ldots, P_{n}\right)$;

(iii) $(X, U, P)$ satisfaz as condiçôes:

$$
\begin{aligned}
& \left.X\right|_{\{0\} \times V}=1_{V} \quad \text { em } \quad \mathcal{G}\left(V ; \mathbb{R}^{n}\right), \\
& \left.P\right|_{\{0\} \times V}=\left.\nabla f\right|_{V} \quad \text { em } \quad \mathcal{G}\left(V ; \mathbb{R}^{n}\right), \\
& \left.U\right|_{\{0\} \times V}=\left.f\right|_{V} \quad \text { em } \quad \mathcal{G}(V ; \mathbb{R}) ;
\end{aligned}
$$

(iv) a função $Y=(\pi, X)$ pertence a $\mathcal{G}_{*}(J \times V ; W)$ e é uma aplicação inversível.

A.2 Observação. Sejam $I, I^{\prime}, \Omega, \Omega^{\prime}, H, f, J$ e $W$ como em A.1. Se existe um representante $\widehat{H}$ de $H$ tal que $\frac{\partial \widehat{H}}{\partial y}=0$ e se $y_{o} \in I^{\prime}, \widehat{H}_{1}$ é a função moderada definida em $] 0,1] \times I \times \Omega \times \Omega^{\prime}$ por

$$
\widehat{H}_{1}(\varepsilon, t, x, p)=\widehat{H}\left(\varepsilon, t, x, y_{o}, p\right)
$$


e $H_{1}$ é a classe de $\widehat{H}_{1}$ em $\mathcal{G}\left(I \times \Omega \times \Omega^{\prime} ; \mathbb{R}\right)$ tem-se que, se $(X, U, P) \in \mathcal{S}_{1}\left(I, \Omega, I^{\prime}, \Omega^{\prime}, H, f, J, W\right)$, então $(X, P) \in \mathcal{S}\left(I, \Omega, \Omega^{\prime}, H_{1}, f, J, W\right)$ e $U$ é como em 2.1.3.

A solução que encontramos para o problema $\mathbf{A}$, que generaliza 2.1.2, é o seguinte teorema:

A.3 Teorema. Sejam $I$ um iritervalo aberto de $\mathbb{R}$ com $0 \in I, I^{\prime}$ um intervalo aberto de $\mathbb{R}, \Omega$ e $\Omega^{\prime}$ abertos de $\mathbb{R}^{n}, H \in \mathcal{G}\left(I \times \Omega \times I^{\prime} \times \Omega^{\prime} ; \mathbb{R}\right)$ e $f \in \mathcal{G}(\Omega ; \mathbb{R})$. Se $J$ é um intervalo aberto de $\mathbb{R}$ com $0 \in J \subset I, W$ um aberto de $\mathbb{R}^{n+1}$ com $V=\left\{z \in \mathbb{R}^{n}:(0, z) \in W\right\} \neq \emptyset$ $e W \subset I \times \Omega$ ese

(i) $\mathcal{S}_{1}\left(I, \Omega, I^{\prime}, \Omega^{\prime}, H, f, J, W\right) \neq \emptyset$;

(ii) $\frac{\partial H}{\partial y}$ tem a propriedade (CLL) em $I \times \Omega \times I^{\prime} \times \Omega^{\prime}$, então o problema A tem uma solução em $\mathcal{G}(W ; \mathbb{R})$.

Demonstração. Denotaremos por $(s, r)=\left(s, r_{1}, \ldots, r_{n}\right)$ um ponto genérico de $J \times V$ e por $(t, x, y, p)=\left(t, x_{1}, \ldots, x_{n}, y, p_{1}, \ldots, p_{n}\right)$ um ponto genérico de $I \times \Omega \times I^{\prime} \times \Omega^{\prime}$.

Sejam $X=\left(X_{1}, \ldots, X_{n}\right), U$ e $P=\left(P_{1}, \ldots, P_{n}\right)$ tais que

$$
(X, U, P) \in \mathcal{S}_{1}\left(I, \Omega, I^{\prime}, \Omega^{\prime}, H, f, J, W\right)
$$

e seja $u=U \circ Y^{-1}$, onde $Y=(\pi, X)$.

Com prova análoga à de 2.1.2 temos que

$$
\begin{aligned}
& \left.u\right|_{\{0\} \times V}=\left.f\right|_{V} \quad \text { em } \mathcal{G}(V ; \mathbb{R}) ; \\
& \left(\frac{\partial u}{\partial t} \circ Y\right)+H \circ(\pi, X, U, P)+\sum_{j=1}^{n}\left(\frac{\partial u}{\partial x_{j}} \circ Y-P_{j}\right) \frac{\partial X_{j}}{\partial s}=0 . \\
& \frac{\partial U}{\partial r_{i}}=\sum_{j=1}^{n}\left(\frac{\partial u}{\partial x_{j}} \circ Y\right) \frac{\partial X_{j}}{\partial r_{i}}, \text { para todo } 1 \leq i \leq n .
\end{aligned}
$$

Fixemos $i \in\{1, \ldots, n\}$ e seja

$$
g=\frac{\partial U}{\partial r_{i}}-\sum_{j=1}^{n} P_{j} \frac{\partial X_{j}}{\partial r_{i}} \quad \text { em } \mathcal{G}(J \times V ; \mathbb{R}) .
$$


Usando A.1.ii e A.1.iii concluímos

$$
\frac{\partial g}{\partial s}=-g \frac{\partial H}{\partial y} \circ(\pi, X, U, P) \quad \text { e }\left.\quad g\right|_{\{0\} \times V}=0 .
$$

Provaremos, a seguir, que $g=0$ em $\mathcal{G}(J \times V ; \mathbb{R})$.

$\operatorname{Sejam}\left(J_{j}\right)_{j \in N}$ uma seqüência exaustiva de compactos para $J \operatorname{com} 0 \in \cap_{j \in N} J_{j}$ e $J_{j}$ intervalo fechado para todo $j \in \mathbb{N},\left(K_{j}^{\prime}\right)_{j \in N}$ uma seqüência exaustiva de compactos para $V, \widehat{X}, \widehat{U}, \widehat{P}$ e $\widehat{H}$ representantes, respectivamente, de $X, U, P$ e $H$.

Fixemos $j \in \mathbb{N}$.

Usando A.1.i existem $K \subset \subset \Omega, J^{\prime} \subset \subset I^{\prime}, K^{\prime \prime} \subset \subset \Omega^{\prime}$ e $\left.\left.\eta_{j} \in\right] 0,1\right]$ tais que

$$
(\widehat{X}, \widehat{U}, \widehat{P})(] 0, \eta_{j}\left[\times J_{j} \times K_{j}^{\prime}\right) \subset K \times J^{\prime} \times K^{\prime \prime} \subset \subset \Omega \times I^{\prime} \times \Omega^{\prime},
$$

e assim existe $\hat{l} \in \mathcal{E}_{M}\left[\stackrel{\circ}{J}_{j} \times \mathbb{R} \times \stackrel{\circ}{K}_{j}^{\prime} ; \mathbb{R}\right]$ tal que

$$
\widehat{l}(\varepsilon, s, t, r)=-t \frac{\partial \widehat{H}}{\partial y}(\varepsilon, s, \widehat{X}(\varepsilon, s, r), \widehat{U}(\varepsilon, s, r), \widehat{P}(\varepsilon, s, r))
$$

em $] 0, \eta_{j}\left[\times \stackrel{\circ}{J}_{j} \times \mathbb{R} \times \stackrel{\circ}{K_{j}^{\prime}}\right.$.

Usando (3), (4) e (ii) concluímos que $\left.g\right|_{J_{j} \times K_{j}^{\prime}}=0$, para todo $j \in \mathbb{N}$, e assim $g=0$. De fato, basta proceder como na prova de 3.2.17 (não podemos usar diretamente 3.2.17 porque não sabemos se $\left.g \in \mathcal{G}_{*}(J \times V ; \mathbb{R})\right)$ observando que dados $q \in \mathbb{N},[a, b] \subset \subset \stackrel{\circ}{J}_{j}$ com $0 \in[a, b], K^{\prime} \subset \subset \stackrel{\circ}{K_{j}^{\prime}}$ e $\alpha \in \mathbb{N}^{n+2}$ podemos, por (ii) e (4), encontrar $N \in \mathbb{N}, \bar{c}>0$ e $\tau \in] 0, \eta_{j}[$ tais que

$$
\begin{aligned}
& \left|\frac{\partial \widehat{l}}{\partial t}(\varepsilon, s, \widehat{g}(\varepsilon, s, r), r)\right| \leq \ln \left(\bar{c} \varepsilon^{-N}\right) ; \\
& |\widehat{l}(\varepsilon, s, \widehat{g}(\varepsilon, s, r), r)-\widehat{l}(\varepsilon, s, 0, r)| \leq \ln \left(\bar{c} \varepsilon^{-N}\right)|\widehat{g}(\varepsilon, s, r)| ; \\
& \left|\partial^{\alpha} \widehat{l}(\varepsilon, s, \widehat{g}(\varepsilon, s, r), r)\right| \leq \bar{c} \varepsilon^{-N} ; \\
& \left|\partial^{\alpha} \widehat{l}(\varepsilon, s, \widehat{g}(\varepsilon, s, r), r)-\partial^{\alpha} \widehat{l}(\varepsilon, s, 0, r)\right| \leq \bar{c} \varepsilon^{-N}|\widehat{g}(\varepsilon, s, r)|,
\end{aligned}
$$

para todo $(\varepsilon, s, r) \in] 0, \tau\left[\times[a, b] \times K^{\prime}\right.$.

Como $g=0$ em $\mathcal{G}(J \times V ; \mathbb{R})$ temos que

$$
\frac{\partial U}{\partial r_{i}}=\sum_{j=1}^{n} P_{j} \frac{\partial X_{j}}{\partial r_{i}}, \text { para todo } 1 \leq i \leq n .
$$


De (1), (2) e (5) obtemos o seguinte sistema:

$$
\begin{aligned}
& {\left[\left(\frac{\partial u}{\partial t} \circ Y\right)+H \circ(\pi, X, U, P)\right]+\left[\left(\frac{\partial u}{\partial x_{1}} \circ Y\right)-P_{1}\right] \frac{\partial X_{1}}{\partial s}+\ldots+\left[\left(\frac{\partial u}{\partial x_{n}} \circ Y\right)-P_{n}\right] \frac{\partial X_{n}}{\partial s}=0} \\
& {\left[\left(\frac{\partial u}{\partial x_{1}} \circ Y\right)-P_{1}\right] \frac{\partial X_{1}}{\partial r_{1}}+\ldots+\left[\left(\frac{\partial u}{\partial x_{n}} \circ Y\right)-P_{n}\right] \frac{\partial X_{n}}{\partial r_{1}}=0} \\
& {\left[\left(\frac{\partial u}{\partial x_{1}} \circ Y\right)-P_{1}\right] \frac{\partial X_{1}}{\partial r_{n}}+\ldots+\left[\left(\frac{\partial u}{\partial x_{n}} \circ Y\right)-P_{n}\right] \frac{\partial X_{n}}{\partial r_{n}}:=0}
\end{aligned}
$$

Úsando que $Y$ é uma aplicação inversível temos, por 1.2.12, que $J Y$ tem inverso multiplicativo em $\mathcal{G}(J \times V ; \mathbb{R})$, e assim por 1.1 .34 concluímos que o sistema acima admite somente a solução trivial, isto é,

$$
\begin{aligned}
& \frac{\partial u}{\partial t} \circ Y=-H \circ(\pi, X,(I, P) \\
& \frac{\partial u}{\partial x_{j}} \circ Y=P_{j}, \quad \text { para todo } 1 \leq j \leq n
\end{aligned}
$$

e portanto

$$
\left(\frac{\partial u}{\partial x_{1}}, \ldots, \frac{\partial u}{\partial x_{n}}\right)=P \circ Y^{-1} \in \mathcal{G}_{*}\left(W ; \Omega^{\prime}\right)
$$

$\mathrm{e}$

$$
\begin{aligned}
\frac{\partial u}{\partial t} & =-H \circ(\pi, X, U, P) \circ Y^{-1}=-H \circ\left(\pi, X, U, \frac{\partial u}{\partial x_{1}} \circ Y, \ldots, \frac{\partial u}{\partial x_{n}} \circ Y\right) \circ Y^{-1} \\
& =-H \circ\left(Y, U, \frac{\partial u}{\partial x_{1}} \circ Y, \ldots, \frac{\partial u}{\partial x_{n}} \circ Y\right) \circ Y^{-1} \\
& =-H \circ\left(\pi, \pi_{1}, \ldots, \pi_{n}, u, \frac{\partial u}{\partial x_{1}}, \ldots, \frac{\partial u}{\partial x_{n}}\right) \cdot / /
\end{aligned}
$$

Analisando a prova de A.3 obtemos o seguinte resultado:

A.4 Proposição. Sejam $I, I^{\prime}, \Omega, \Omega^{\prime}, H, f, J$ e $W$ como em A.3. Se $(X, U, P) \in$ $\mathcal{S}_{1}\left(I, \Omega, I^{\prime}, \Omega^{\prime}, H, f, J, W\right)$ e $Y=(\pi, X)$, então

(I) $u=U \circ Y^{-1}$ é uma solução para o problema $\mathbf{A}$ em $\mathcal{G}(W ; \mathbb{R})$;

(II) denotando por $(t, x)=\left(t, x_{1}, \ldots, x_{n}\right)$ um ponto genérico de $W$, tem-se que

$$
\left(\frac{\partial u}{\partial x_{1}}, \ldots, \frac{\partial u}{\partial x_{n}}\right)=P \circ Y^{-1} \text {. }
$$


Apresentamos a seguir os resultados que obtivemos quanto à unicidade de soluções para o problema $\mathbf{A}$.

A.5 Teorema. Sejam $\Omega$ e $\Omega^{\prime}$ abertos de $\mathbb{R}^{n}, I$ e $J$ intervalos abertos de $\mathbb{R}$ com $0 \in$ $J \subset I, I^{\prime}$ um intervalo aberto de $\mathbb{R}, W$ um aberto de $\mathbb{R}^{n+1}$ com $V=\left\{z \in \mathbb{R}^{n}:(0, z) \in\right.$ $W\} \neq \emptyset$ e $W \subset I \times \Omega, H \in \mathcal{G}\left(I \times \Omega \times I^{\prime} \times \Omega^{\prime} ; \mathbb{R}\right)$ e $f \in \mathcal{G}(\Omega ; \mathbb{R})$. Se existe um representante $\widehat{H}$ de $H$ tal que

(i) $\frac{\partial^{2} \widehat{H}}{\partial x_{j} \partial p_{i}}, \frac{\partial^{2} \widehat{H}}{\partial x_{j} \partial x_{i}}, \frac{\partial^{2} \widehat{H}}{\partial p_{j} \partial p_{i}}, \frac{\partial^{2} \widehat{H}}{\partial x_{j} \partial y}, \frac{\partial^{2} \widehat{H}}{\partial y^{2}}$ e $\frac{\partial^{2} \widehat{H}}{\partial p_{j} \partial y}$ têm a propriedade (CLL) em $I \times \Omega \times I^{\prime} \times \Omega^{\prime}$, para todo $1 \leq i, j \leq n$;

(ii) $\frac{\partial \widehat{H}}{\partial x_{j}}, \frac{\partial \widehat{H}}{\partial y}$ e $\frac{\partial \widehat{H}}{\partial p_{j}}$ têm a propriedade (CLL) em $I \times \Omega \times I^{\prime} \times \Omega^{\prime}$ para todo $1 \leq j \leq n$, ou $\frac{\partial \widehat{H}}{\partial y}=0$;

(iii) $\frac{\partial \widehat{H}}{\partial x}, \frac{\partial \widehat{H}}{\partial y} \quad e \frac{\partial \widehat{H}}{\partial p}$ têm a propriedade (LLL) em $\left(I, \Omega \times I^{\prime} \times \Omega^{\prime}\right)$;

(iv) $\widehat{H}$ tem a propriedade (LLL) em $\left(I, \Omega \times I^{\prime} \times \Omega^{\prime}\right)$ ou $\frac{\partial \widehat{H}}{\partial y}=0$, então $\operatorname{card} \mathcal{S}_{1}\left(I, \Omega, I^{\prime}, \Omega^{\prime}, H, f, J, W\right) \leq 1$.

Demonstração. Se $\frac{\partial \widehat{H}}{\partial y}=0$ temos, por A.2 e 3.3.3, que

$$
\operatorname{card} \mathcal{S}_{1}\left(I, \Omega, I^{\prime}, \Omega^{\prime}, H, f, J, W\right) \leq \mathcal{S}\left(I, \Omega, \Omega^{\prime}, H_{1}, f, J, W\right) \leq 1,
$$

sendo $H_{1}$ como em A.2.

Se $\frac{\partial \widehat{H}}{\partial y} \neq 0$, a prova é análoga à de 3.3 .3 tomando para $\widehat{\varphi}=\left(\varphi_{1}, \ldots, \varphi_{2 n+1}\right)$ a aplicação moderada definida em $] 0,1] \times J \times\left(\Omega \times I^{\prime} \times \Omega^{\prime}\right) \times V$ por

$$
\begin{aligned}
& \left(\widehat{\varphi}_{1}, \ldots, \widehat{\varphi}_{n}\right)(\varepsilon, t, x, y, p, r)=\frac{\partial \widehat{H}}{\partial p}(\varepsilon, t, x, y, p) \\
& \left(\widehat{\varphi}_{n+1}, \ldots, \widehat{\varphi}_{2 n}\right)(\varepsilon, t, x, y, p, r)=-\frac{\partial \widehat{H}}{\partial x}(\varepsilon, t, x, y, p)-p \frac{\partial \widehat{H}}{\partial y}(\varepsilon, t, x, y, p), \\
& \hat{\varphi}_{2 n+1}(\varepsilon, t, x, y, p, r)=-\widehat{H}(\varepsilon, t, x, y, p)+\sum_{j=1}^{n} p_{j} \frac{\partial \widehat{H}}{\partial p_{j}}(\varepsilon, t, x, y, p)
\end{aligned}
$$


e observando que se $(X, U, P)$ e $(\tilde{X}, \tilde{U}, \tilde{P})$ pertencem a $\mathcal{S}_{1}\left(I, \Omega, I^{\prime}, \Omega^{\prime}, H, f, J, W\right)$, então $(X, U, P)$ e $(\tilde{X}, \tilde{U}, \tilde{P})$ são soluções da equação nas incógnitas $(\bar{X}, \bar{U}, \bar{P})$

$$
\left(\frac{\partial \bar{X}}{\partial s}, \frac{\partial \bar{U}}{\partial s}, \frac{\partial \bar{P}}{\partial s}\right)=\varphi \circ\left(\pi, \bar{X}, \bar{U}, \bar{P}, \pi_{1}, \ldots, \pi_{n}\right)
$$

com a condição

$$
\left.(\bar{X}, \bar{U}, \bar{P})\right|_{\{0\} \times V}=\left(1_{V},\left.f\right|_{V},\left.\nabla f\right|_{V}\right) \cdot / /
$$

A.6 Teorema. Sejam $\Omega^{\prime}$ um aberto de $\mathbb{R}^{n}, I$ e $J$ intervalos abertos de $\mathbb{R}$ com $0 \in J \subset I$, $H \in \mathcal{G}\left(I \times \mathbb{R}^{n} \times \mathbb{R} \times \Omega^{\prime} ; \mathbb{R}\right), \widehat{H}$ um representante de $H$ e $f \in \mathcal{G}\left(\mathbb{R}^{n} ; \mathbb{R}\right)$ tais que $\mathcal{S}_{1}\left(I, \mathbb{R}^{n}, \mathbb{R}, \Omega^{\prime}, H, f, J, J \times \mathbb{R}^{n}\right) \neq \emptyset$. Sc

(i) $\frac{\partial \widehat{H}}{\partial p}$ é uma aplicação limitada em $I \times \mathbb{R}^{n} \times \mathbb{R} \times \Omega^{\prime}$;

(ii) $\widehat{H}$ satisfaz de A.5.i até A.5.iv (substituindo $\Omega$ por $\mathbb{R}^{n}$ e $I^{\prime}$ por $\mathbb{R}$ ), se $(X, U, P) \in \mathcal{S}_{1}\left(I, \mathbb{R}^{n}, \mathbb{R}, \Omega^{\prime}, H, f, J, J \times \mathbb{R}^{n}\right)$ e se existe uma função $v \in \mathcal{G}\left(J \times \mathbb{R}^{n} ; \mathbb{R}\right)$ satisfazendo

(iii) $v$ é uma solução para o problema $\mathbf{A}$ em $\mathcal{G}\left(J \times \mathbb{R}^{n} ; \mathbb{R}\right)$;

(iv) existem um representante $\hat{v}$ de $v$ e $\tau \in] 0,1]$ tais que

$$
\left(\frac{\partial \widehat{v}}{\partial x_{1}}, \ldots, \frac{\partial \widehat{v}}{\partial x_{n}}\right)(] 0, \tau\left[\times J \times \mathbb{R}^{n}\right) \subset \Omega^{\prime}
$$

onde $(t, x)=\left(t, x_{1}, \ldots, x_{n}\right)$ denota um ponto genérico de $J \times \mathbb{R}^{n}$;

(v) $\partial^{\alpha} v \in \mathcal{G}_{*}\left(J \times \mathbb{R}^{n} ; \mathbb{R}\right)$, para todo $\alpha=\left(\alpha_{0}, \alpha_{1}, \ldots, \alpha_{n}\right) \in \mathbb{N}^{n+1}$ com $\alpha_{0}=0<$ $|\alpha|=2$,

então $v=U \circ Y^{-1}$, sendo $Y=(\pi, X)$.

Demonstração. Se $\frac{\partial \widehat{H}}{\partial y}=0$ basta usar A.2 e 3.3.4. Suponhamos $\frac{\partial \widehat{H}}{\partial y} \neq 0$. A prova é análoga à de 3.3.4 tomando para $\hat{\varphi}$ uma aplicação definida em $] 0,1] \times J \times \mathbb{R}^{n} \times \mathbb{R}^{n}$ tal que em $] 0, \tau\left[\times J \times \mathbb{R}^{n} \times \mathbb{R}^{n}\right.$ tem-se

$$
\widehat{\varphi}(\varepsilon, t, x, r)=\frac{\partial \widehat{H}}{\partial p}\left(\varepsilon, t, x, \widehat{v}(\varepsilon, t, x), \frac{\partial \widehat{v}}{\partial x_{1}}(\varepsilon, t, x), \ldots, \frac{\partial \widehat{v}}{\partial x_{n}}(\varepsilon, t, x)\right) ;
$$


substituindo (2) pela equação de incógnita $\tilde{X}$

$$
\frac{\partial \tilde{X}}{\partial s}=\varphi \circ\left(\pi, \tilde{X}, \pi_{1}, \ldots, \pi_{n}\right)=\frac{\partial H}{\partial p} \circ\left(\pi, \tilde{X}, v \circ(\pi, \tilde{X}), \frac{\partial v}{\partial x_{1}} \circ(\pi, \tilde{X}), \ldots, \frac{\partial v}{\partial x_{n}} \circ(\pi, \tilde{X})\right) ;
$$

tomando para $\hat{\Psi}=\left(\widehat{\Psi}_{1}, \ldots, \Psi_{2 n+1}\right)$ a aplicação moderada definida em $\left.] 0,1\right] \times J \times \mathbb{R}^{n} \times \mathbb{R} \times$ $\Omega^{\prime} \times \mathbb{R}^{n}$ por

$$
\begin{aligned}
& \left(\widehat{\Psi}_{1}, \ldots, \widehat{\Psi}_{n}\right)(\varepsilon, s, x, y, p, r)=\frac{\partial \widehat{H}}{\partial p}(\varepsilon, s, x, y, p), \\
& \left(\widehat{\Psi}_{n+1}, \ldots, \widehat{\Psi}_{2 n}\right)(\varepsilon, s, x, y, p, r)=-\frac{\partial \widehat{H}}{\partial x}(\varepsilon, s, x, y, p)-p \frac{\partial \widehat{H}}{\partial y}(\varepsilon, s, x, y, p), \\
& \widehat{\Psi}_{2 n+1}(\varepsilon, s, x, y, p, r)=-\widehat{H}(\varepsilon, s, x, y, p)+\sum_{j=1}^{n} p_{j} \frac{\partial \widehat{H}}{\partial p_{j}}(\varepsilon, s, x, y, p)
\end{aligned}
$$

e observando que, se $\tilde{U}=v \circ(\pi, \tilde{X})$ então $(\tilde{X}, \tilde{U}, \tilde{P})$ é uma solução, em $\mathcal{G}\left(J \times \mathbb{R}^{n} ; \mathbb{R}^{2 n+1}\right)$, do sistema de incógnitas $\bar{X}, \bar{U}$ e $\bar{P}$

$$
\begin{aligned}
& \frac{\partial \bar{X}}{\partial s}=\frac{\partial H}{\partial p} \circ(\pi, \bar{X}, \bar{U}, \bar{P}) \\
& \frac{\partial \bar{P}}{\partial s}=-\frac{\partial H}{\partial x} \circ(\pi, \bar{X}, \bar{U}, \bar{P})-\bar{P} \frac{\partial H}{\partial y} \circ(\pi, \bar{X}, \bar{U}, \bar{P}) \\
& \frac{\partial \bar{U}}{\partial s}=-H \circ(\pi, \bar{X}, \bar{U}, \bar{P})+\sum_{j=1}^{n} \frac{\partial H}{\partial p_{j}} \circ(\pi, \bar{X}, \bar{U}, \bar{P}) \bar{P}_{j} ;
\end{aligned}
$$

com a condição

$$
\left.(\bar{X}, \bar{U}, \bar{P})\right|_{\{0\} \times V}=\left(1_{R^{n}}, f, \nabla f\right) \cdot / /
$$

A.7 Teorema. Sejam $I$ e $J$ intervalos abertos de $\mathbb{R}$ com $0 \in J \subset I, H \in \mathcal{G}\left(I \times \mathbb{R}^{n} \times \mathbb{R} \times\right.$ $\left.\mathbb{R}^{n} ; \mathbb{R}\right), \widehat{H}$ um representante de $H$ e $f \in \mathcal{G}\left(\mathbb{R}^{n} ; \mathbb{R}\right)$ tais que $\mathcal{S}_{1}\left(I, \mathbb{R}^{n}, \mathbb{R}, \mathbb{R}^{n}, H, f, J, J \times\right.$ $\left.\mathbb{R}^{n}\right) \neq \emptyset$. Denotando por $B_{r}$ a bola aberta de centro 0 e raio $r$ em $\mathbb{R}^{n}$ tem-se que, se

(i) existem $M>0$ e $\tau \in] 0,1]$ tais que $\frac{\partial \widehat{H}}{\partial p}(] 0, \tau\left[\times I \times \overline{B_{r}} \times \overline{I_{r}} \times \overline{B_{r}}\right) \subset \overline{B_{r M}}$, para todo $r>0$;

(ii) $\widehat{H}$ satisfaz de A.5.i até A.5.iv (substituindo $\Omega$ e $\Omega^{\prime}$ por $\mathbb{R}^{n}$ e $I^{\prime}$ por $\mathbb{R}$ ), se $(X, U, P) \in \mathcal{S}_{1}\left(I, \mathbb{R}^{n}, \mathbb{R}, \mathbb{R}^{n}, H, f, J, J \times \mathbb{R}^{n}\right)$ e se existe uma função $v \in \mathcal{G}\left(J \times \mathbb{R}^{n} ; \mathbb{R}\right)$ satisfazendo 
(iii) $v$ é uma solução para o problema $\mathbf{A}$ em $\mathcal{G}\left(J \times \mathbb{R}^{n} ; \mathbb{R}\right)$;

(iv) existem um representante $\hat{v}$ de $v, M_{1}>0$ e $\left.\left.\tau_{1} \in\right] 0,1\right]$ tais que para todo $r>0$

e $\alpha=\left(\alpha_{0}, \alpha_{1}, \ldots, \alpha_{n}\right) \in N^{n+1}$ com $\alpha_{0}=0<|\alpha|=1$, tem-se que

$$
\left(\widehat{v}, \partial^{\alpha} \widehat{v}\right)(] 0, \tau_{1}\left[\times J \times \overline{B_{r}}\right) \subset \overline{I_{r M_{1}}} \times \overline{B_{r M_{1}}}
$$

(iv) $\partial^{\alpha} v \in \mathcal{G}_{*}\left(J \times \mathbb{R}^{n} ; \mathbb{R}\right)$, para todo $\alpha=\left(\alpha_{0}, \alpha_{1}, \ldots, \alpha_{n}\right) \in \mathbb{N}^{n+1}$ com $\alpha_{0}=0<$ $|\alpha|=2$,

então $v=U \circ Y^{-1}$, sendo $Y=(\pi, X)$.

Demonstração. Análoga à de 3.3.7 substituindo, em 3.3.7, 3.3.4 por A.6. // 


\section{Bibliografia}

[1] J. ARAGONA, Introdução à Teoria das Funçôes Generalizadas de Colombeau, IMEUSP, São Paulo, 1989(Notas de aula da disciplina MAT829, Instituto de Matemática e Estatística da Universidade de São Paulo).

[2] J. ARAGONA and H. BIAGIONI, Intrinsic definition of the Colombeau algebra of generalized functions, Anal. Math., 17(1991), 75-132.

[3] J. ARAGONA and F. VILLARREAL, Colombeau's theory and shock waves in a problem of hydrodynamics, J. Analyse Math., 61(1993), 113-144.

[4] S. H. BENTON The Hamilton-Jacobi equation: A global approach, Academic Press, New York, 1977.

[5] H. A. BIAGIONI, A nonlinear Theory of Generalized functions, Lecture Notes in Math. 1421, Springer, New York, 1990.

[6] H. A. BIAGIONI, Generalized solutions to nonlinear first-order systems, Monatsh. Math., 118(1994), 7-20.

[7] J. F. COLOMBEAU, The Cauchy problem in a space of generalized functions I, C. $R$. Acad. Sci. Paris Sér. I Math., 317(1993), 851-855.

[8] M. G. CRANDALL and P. L. LIONS, Viscosity solutions of Hamilton-Jacobi equations, Trans. Amer. Math. Soc., 277(1)(1983), 1-42. 
[9] M. G. CRANDALL, L. C. EVANS and P. L. LIONS, Some properties of viscosity solutions of Hamilton-Jacobi equations, Trans. Amer. Math. Soc., 282(2)(1984), 487502.

[10] J. DIEUDONnÉ, Foudations of Modern Analysis, Academic Press, New York, 1960.

[11] C. S. HÖNIG, Aplicações da Topologia à Análise, IMPA, Rio de Janeiro, 1976.

[12] M. W. OLIVA, Equações diferenciais ordinárias, IME-USP, São Paulo, 1971(Publicação do Instituto de Matemática e Estatística da Universidade de São Paulo).

[13] L. E. SIGLER, Algebra, Graduate Texts in Mathematics, Springer, New York, 1976.

[14] F. VILLARREAL, Sobre soluções na forma de onda de choque de certos sistemas de equações diferenciais parciais na hidrodinâmica, IME-USP, São Paulo, 1990(Tese de Doutorado, Universidade de São Paulo). 